\title{
Skeletal muscle mitochondrial clearance
}

Citation for published version (APA):

Leermakers, P. A. (2019). Skeletal muscle mitochondrial clearance: translation to COPD. [Doctoral Thesis, Maastricht University]. ProefschriftMaken Maastricht. https://doi.org/10.26481/dis.20190405pl

Document status and date:

Published: 01/01/2019

DOI:

10.26481/dis.20190405pl

Document Version:

Publisher's PDF, also known as Version of record

\section{Please check the document version of this publication:}

- A submitted manuscript is the version of the article upon submission and before peer-review. There can be important differences between the submitted version and the official published version of record.

People interested in the research are advised to contact the author for the final version of the publication, or visit the DOI to the publisher's website.

- The final author version and the galley proof are versions of the publication after peer review.

- The final published version features the final layout of the paper including the volume, issue and page numbers.

Link to publication

\footnotetext{
General rights rights.

- You may freely distribute the URL identifying the publication in the public portal. please follow below link for the End User Agreement:

www.umlib.nl/taverne-license

Take down policy

If you believe that this document breaches copyright please contact us at:

repository@maastrichtuniversity.nl

providing details and we will investigate your claim.
}

Copyright and moral rights for the publications made accessible in the public portal are retained by the authors and/or other copyright owners and it is a condition of accessing publications that users recognise and abide by the legal requirements associated with these

- Users may download and print one copy of any publication from the public portal for the purpose of private study or research.

- You may not further distribute the material or use it for any profit-making activity or commercial gain

If the publication is distributed under the terms of Article $25 \mathrm{fa}$ of the Dutch Copyright Act, indicated by the "Taverne" license above, 


\section{Skeletal muscle mitochondrial clearance: translation to COPD}

Pieter Arnold Leermakers 


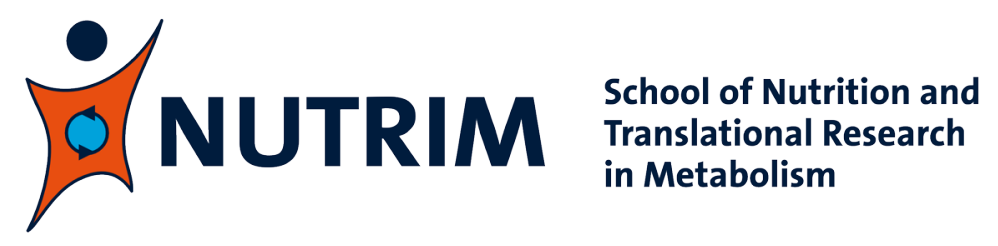

The studies presented in this thesis were performed at the NUTRIM School of Nutrition and Translational Research in Metabolism. These studies were supported by a grant from the NUTRIM Graduate Programme.

Cover design: Pieter Leermakers

Cover image: front; C2C12 cells exposed to $300 \mu \mathrm{m}$ DFP for $24 \mathrm{~h}$. back; murine gastrocnemius muscle.

Layout: Judith Leermakers-Ceelen

Printed by: proefschriftmaken.nl

ISBN: $\quad$ 978-94-6380-270-3

(c) Copyright Pieter A. Leermakers, Maastricht 2019. 


\title{
Skeletal muscle mitochondrial clearance: translation to COPD
}

\author{
Proefschrift
}

Ter verkrijging van de graad van doctor aan de Universiteit Maastricht, op het gezag van de Rector Magnificus, Prof. dr. Rianne M. Letschert, volgens het besluit van het College van Decanen, in het openbaar te verdedigen op vrijdag

5 april 2019 om 14.00 uur.

door

Pieter Arnold Leermakers

Geboren te Eindhoven op 20 december 1988, Nederland 


\section{Promotor}

Prof. dr. A.M.W.J. Schols

\section{Co-promotor}

Dr. H.R. Gosker

\section{Beoordelingscommissie}

Prof. dr. E.E. Blaak (voorzitter)

Prof. dr. H.I. Heijink (Universitair Medisch Centrum Groningen, Nederland)

Dr. J. Hoeks

Prof. dr. F-J. van Schooten

Prof. dr. J.A.M. Smeitink (Radboud Universitair Medisch Centrum) 


\section{Contents}

$\begin{array}{lll}\text { Chapter } 1 & \text { General introduction } & 7\end{array}$

Chapter 2 Skeletal muscle mitophagy in chronic disease: 15

implications for muscle oxidative capacity?

Chapter 3 Molecular signalling towards mitochondrial breakdown is enhanced in skeletal muscle of patients with chronic obstructive pulmonary disease (COPD)

Chapter 4 Inflammation-induced alterations in key regulators of

61 mitophagy and mitochondrial biogenesis in murine skeletal muscle

Chapter 5 Early-onset alterations in mitophagy and mitochondrial 91 biogenesis signalling during skeletal muscle unloading in mice and men

Chapter 6 Coordinated regulation of skeletal muscle mass and metabolic plasticity during recovery from disuse

Chapter 7 Iron deficiency-induced loss of skeletal muscle mitchondria; the role of mitophagy and secretion of mitochondria-containing vesicles

Chapter 8 General discussion

Chapter 9 Summary

Nederlandse samenvatting $\quad 197$

Valorisation 205

Dankwoord 213

Publications $\quad 221$

Curriculum Vitae $\quad 225$ 

1

General introduction 


\section{Introduction}

Chronic obstructive pulmonary disease (COPD) is a highly prevalent chronic disease, affecting over 65 million patients worldwide, which causes over 3 million deaths on a yearly basis $(1,2)$. COPD patients suffer from airflow limitation, caused by emphysema, chronic bronchitis or both, of which the main risk factors include tobacco smoke, air pollution and occupational hazards (3). Interestingly, COPD patients are also characterized by skeletal muscle weakness, which results in reduced exercise capacity, and subsequently contributes to increased morbidity of COPD (4). Important and well established drivers of skeletal muscle weakness in COPD include loss of skeletal muscle mass and oxidative capacity. More specifically regarding this loss of oxidative capacity, previous studies established that skeletal muscle of COPD patients were not only characterized by a fibre-type shift from oxidative to glycolytic fibres, but also by several mitochondrial impairments, such as decreased expression of mitochondrial components, decreased oxidative enzyme activity, decreased mitochondrial fractional area, and increased mitochondrial ROS production (4-8).

Mitochondria are highly dynamic organelles, constantly changing shape and size, and undergoing clearance and biogenesis. These processes form a mitochondrial homeostasis in healthy conditions, but a relative shift favouring mitochondrial clearance can result in mitochondrial impairments or loss of mitochondrial content (9). The main focus of research aimed to elucidate the regulation of impaired skeletal muscle oxidative metabolism in patients with COPD has been on impaired skeletal muscle mitochondrial biogenesis to date $(5,10,11)$, but remarkably, the role of mitochondrial clearance remains understudied (12).

There are several routes of mitochondrial clearance, of which mitophagy, defined as a mitochondria-specific form of macro-autophagy (13), is the best described in literature (9). Furthermore, mitochondria can also be cleared via ubiquitin-mediated protein breakdown (9), mitochondrial-derived vesicle formation (9), or via secretion of mitochondria-containing extracellular vesicles $(14,15)$. Research aimed at the role of mitochondrial clearance in loss and recovery of oxidative capacity will result in new insights that may be beneficial in development or optimization of exercise training and oxidative capacity preserving nutritional or pharmacological therapy.

In chapter 2, we conducted a literature study to identify extra-pulmonary manifestations of COPD, including muscle disuse, systemic inflammation, and iron deficiency, that have previously been proposed to be associated with 
the loss of skeletal muscle oxidative capacity $(6,16)$, as possible mitophagyinducing triggers. Since similar skeletal muscle mitochondrial impairments have been reported for congestive heart failure (CHF) and type 2 diabetes (T2D) as well (17-23), we included these disorders in this study as well.

The literature review of chapter 2 led to the overall hypothesis of this thesis: "COPD-related manifestations including muscle disuse, systemic inflammation, and more speculatively, iron deficiency, result in the onset of mitochondrial clearance, and consequently in the loss of muscle oxidative capacity".

In chapter 3, we studied if molecular signalling patterns indicative of increased mitophagy could be identified in vastus lateralis skeletal muscle biopsies of a cohort of COPD patients compared with healthy controls, and whether there were differences in molecular mitophagy signalling between patients with versus without iron deficiency and with high versus low plasma CRP levels. Based on the results of chapters 2 and 3 we subsequently focused on the individual contribution of systemic inflammation, muscle disuse and reloading, and iron deficiency on skeletal muscle mitophagy in different experimental models.

In chapter 4, we used a murine model of pulmonary- induced systemic inflammation initiated by a single bolus of intra-tracheally (IT)-instilled lipopolysaccharide (LPS) to analyse multiple important constituents and molecular regulators of mitophagy and mitochondrial biogenesis in $m$. gastrocnemius over time $(24,48,72,96$, and $120 \mathrm{~h})$.

In chapter 5, we used a murine model of complete skeletal muscle disuse, in which mice were subjected to 3 days of hind-limb suspension (HLS), to analyse multiple important constituents and molecular regulators of mitophagy and mitochondrial biogenesis in $m$. gastrocnemius during early stages of skeletal muscle unloading. To validate these results in a human model of partial skeletal muscle disuse, healthy men were subjected to 7 days of one-leg casting, and molecular analyses were performed, similar to the analyses of the murine model, in paired pre- and post-biopsies of $m$. vastus lateralis.

Furthermore, we explored whether recovery of disuse-induced loss of muscle oxidative capacity is associated with altered mitochondrial biogenesis and clearance signalling. Therefore, in chapter 6 , we used the same models for muscle disuse as described in chapter 5 , but added a recovery period to study 
disuse recovery. In the murine model, mice were let to recover for 1, 2, 3, 5 or 8 days after 14 days of HLS, and the human participants were let to recover for 7 days after 7 days of unloading. We first measured molecular profiles of protein turnover, mitochondrial turnover (including mitophagy signalling), and myonuclear turnover in vastus lateralis biopsies of the human model, and performed cluster analysis of the recovery-response. We subsequently analysed these molecular profiles in the more elaborate murine model.

In chapter 7, we investigated the causal relationship between iron chelation and the induction of mitochondrial clearance via mitophagy and mitochondrial secretion. To study this, we subjected in vitro differentiated murine skeletal muscle cells (myotubes) to the iron chelator deferiprone, after which we analysed the process of mitophagy, mitochondrial excretion, and mitochondrial quantity and function. In addition, we inhibited autophagy, and knocked-down specific mitophagy receptors, to determine their involvement in iron chelation induced mitochondrial impairments.

Lastly, the results from the studies described in this thesis are discussed in a broader context, and potential new research topics are identified in chapter $\mathbf{8}$. 


\section{References}

1. WHO. Mortality and global health estimates: Cause-specific mortality [Web Page]. World Health Organisation; 2014 [updated 2013; cited 2014 14-04-2014]. Mortality and global health estimates: Cause-specific mortality]. Available from: http://apps. who.int/gho/data/node.main.GHECOD?lang=en.

2. WHO. The 10 leading causes of death in the world, 2000 and 20122014 [updated 05-2014; cited 2014 13-10-2014]. Available from: http://who.int/mediacentre/ factsheets/fs310/en/.

3. Vogelmeier CF, Criner GJ, Martinez FJ, Anzueto A, Barnes PJ, Bourbeau J, et al. Global Strategy for the Diagnosis, Management, and Prevention of Chronic Obstructive Lung Disease 2017 Report. GOLD Executive Summary. American journal of respiratory and critical care medicine. 2017 Mar 1;195(5):557-82. PubMed PMID: 28128970.

4. Maltais F, Decramer M, Casaburi R, Barreiro E, Burelle $Y$, Debigare R, et al. An official American Thoracic Society/European Respiratory Society statement: update on limb muscle dysfunction in chronic obstructive pulmonary disease. American journal of respiratory and critical care medicine. 2014 May 1;189(9): e15-62. PubMed PMID: 24787074. Pubmed Central PMCID: 4098112.

5. van den Borst B, Slot IG, Hellwig VA, Vosse BA, Kelders MC, Barreiro E, et al. Loss of quadriceps muscle oxidative phenotype and decreased endurance in patients with mild-to-moderate COPD. Journal of applied physiology. 2013 May;114(9):1319-28. PubMed PMID: 22815389.

6. Gosker HR, Wouters EF, van der Vusse GJ, Schols AM. Skeletal muscle dysfunction in chronic obstructive pulmonary disease and chronic heart failure: underlying mechanisms and therapy perspectives. The American journal of clinical nutrition. 2000 May;71(5):1033-47. PubMed PMID: 10799364.

7. Gosker HR, Hesselink MK, Duimel H, Ward KA, Schols AM. Reduced mitochondrial density in the vastus lateralis muscle of patients with COPD. The European respiratory journal. 2007 Jul;30(1):73-9. PubMed PMID: 17428811.

8. Puente-Maestu L, Perez-Parra J, Godoy R, Moreno N, Tejedor A, GonzalezAragoneses $\mathrm{F}$, et al. Abnormal mitochondrial function in locomotor and respiratory muscles of COPD patients. The European respiratory journal. 2009 May;33(5): 1045-52. PubMed PMID: 19129279.

9. Romanello V, Sandri M. Mitochondrial Quality Control and Muscle Mass Maintenance. Frontiers in physiology. 2015;6:422. PubMed PMID: 26793123. Pubmed Central PMCID: 4709858.

10. Konokhova Y, Spendiff S, Jagoe RT, Aare S, Kapchinsky S, MacMillan NJ, et al. Failed upregulation of TFAM protein and mitochondrial DNA in oxidatively deficient fibers of chronic obstructive pulmonary disease locomotor muscle. Skeletal muscle. 2016;6:10. PubMed PMID: 26893822. Pubmed Central PMCID: 4758107.

11. Remels AH, Schrauwen P, Broekhuizen R, Willems J, Kersten S, Gosker HR, et al. Peroxisome proliferator-activated receptor expression is reduced in skeletal muscle in COPD. The European respiratory journal. 2007 Aug;30(2):245-52. PubMed PMID: 17459894. 
12. Guo Y, Gosker HR, Schols AM, Kapchinsky S, Bourbeau J, Sandri M, et al. Autophagy in locomotor muscles of patients with chronic obstructive pulmonary disease. American journal of respiratory and critical care medicine. 2013 Dec 1;188(11):1313-20. PubMed PMID: 24228729.

13. Lemasters JJ. Selective mitochondrial autophagy, or mitophagy, as a targeted defense against oxidative stress, mitochondrial dysfunction, and aging. Rejuvenation research. 2005 Spring;8(1):3-5. PubMed PMID: 15798367.

14. Phinney DG, Di Giuseppe M, Njah J, Sala E, Shiva S, St Croix CM, et al. Mesenchymal stem cells use extracellular vesicles to outsource mitophagy and shuttle microRNAs. Nature communications. 2015 Oct 7;6:8472. PubMed PMID: 26442449. Pubmed Central PMCID: 4598952.

15. Falchi AM, Sogos V, Saba F, Piras M, Congiu T, Piludu M. Astrocytes shed large membrane vesicles that contain mitochondria, lipid droplets and ATP. Histochemistry and cell biology. 2013 Feb;139(2):221-31. PubMed PMID: 23108569.

16. Stugiewicz M, Tkaczyszyn M, Kasztura M, Banasiak W, Ponikowski P, Jankowska EA. The influence of iron deficiency on the functioning of skeletal muscles: experimental evidence and clinical implications. European journal of heart failure. 2016 Jan 21. PubMed PMID: 26800032.

17. Adams V, Doring C, Schuler G. Impact of physical exercise on alterations in the skeletal muscle in patients with chronic heart failure. Frontiers in bioscience : a journal and virtual library. 2008;13:302-11. PubMed PMID: 17981548.

18. Gosker HR, Lencer NH, Franssen FM, van der Vusse GJ, Wouters EF, Schols AM. Striking similarities in systemic factors contributing to decreased exercise capacity in patients with severe chronic heart failure or COPD. Chest. 2003 May;123(5): 1416-24. PubMed PMID: 12740256.

19. Kelley DE, He J, Menshikova EV, Ritov VB. Dysfunction of mitochondria in human skeletal muscle in type 2 diabetes. Diabetes. 2002 Oct;51(10):2944-50. PubMed PMID: 12351431.

20. Kinugawa S, Takada S, Matsushima S, Okita K, Tsutsui H. Skeletal Muscle Abnormalities in Heart Failure. International heart journal. 2015;56(5):475-84. PubMed PMID: 26346520.

21. Lavine KJ, Sierra OL. Skeletal muscle inflammation and atrophy in heart failure. Heart failure reviews. 2017 Mar;22(2):179-89. PubMed PMID: 28091823. Pubmed Central PMCID: 5352459.

22. Mogensen M, Sahlin K, Fernstrom M, Glintborg D, Vind BF, Beck-Nielsen H, et al. Mitochondrial respiration is decreased in skeletal muscle of patients with type 2 diabetes. Diabetes. 2007 Jun;56(6):1592-9. PubMed PMID: 17351150.

23. Phielix E, Schrauwen-Hinderling VB, Mensink M, Lenaers E, Meex R, Hoeks J, et al. Lower intrinsic ADP-stimulated mitochondrial respiration underlies in vivo mitochondrial dysfunction in muscle of male type 2 diabetic patients. Diabetes. 2008 Nov;57(11):2943-9. PubMed PMID: 18678616. Pubmed Central PMCID: 2570390 . 


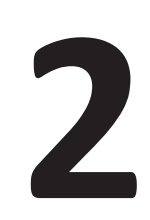

Skeletal muscle mitophagy in chronic disease:implicationsformuscleoxidative capacity?

P.A. Leermakers, H.R. Gosker

Curr Opin Clin Nutr Metab Care. 2016 Nov;19(6):427-433. 


\begin{abstract}

\section{Purpose of review}

Loss of skeletal muscle oxidative capacity is a common feature of chronic diseases such as chronic obstructive pulmonary disease, type 2 diabetes, and congestive heart failure. It may lead to physical impairments and has been suggested to contribute to metabolic inflexibility-induced cardiometabolic risk. The mechanism underlying loss of muscle oxidative capacity is incompletely understood. This review discusses the role of mitophagy as a driving force behind the loss of skeletal muscle oxidative capacity in these patients.
\end{abstract}

\title{
Recent findings
}

Mitophagy has been studied to a very limited extent in human skeletal muscle. There are, however, clear indications that disease-related factors, including hypoxia, systemic inflammation, muscle inactivity, and iron deficiency are able to induce mitophagy, and that these factors trigger mitophagy via different regulatory mechanisms. Although mitophagy may lead to mitochondrial loss, it is also required to maintain homeostasis through clearance of damaged mitochondria.

\section{Summary}

Based on available evidence, we propose that enhanced mitophagy is involved in chronic disease-induced loss of muscle oxidative capacity. Clearly more research is required to confirm this role and to establish to what extent mitophagy is pathological or a part of physiological adaptation to maintain muscle health.

\section{Keywords}

chronic obstructive pulmonary disease, congestive heart failure, mitophagy, skeletal muscle, type 2 diabetes 


\section{Introduction}

Loss of lower limb skeletal muscle oxidative capacity, defined by the ability to oxidize nutrients to obtain energy, is a common feature of chronic diseases such as chronic obstructive pulmonary disease (COPD) (1), type 2 diabetes (T2D) $(2,3)$, and congestive heart failure (CHF) $(4,5)$. Functional impairments, such as muscle dysfunction and decreased exercise capacity, are associated with reduced muscle oxidative capacity (1-5), and eventually may lead to disability or even handicaps. Moreover, impaired muscle oxidative capacity has been proposed to accelerate muscle wasting $(6,7)$ and may lead to metabolic inflexibility and increased cardiometabolic risk (8).

Oxidative capacity is defined by the maximal rate at which oxidative phosphorylation, a mitochondrial-based process in which energy is obtained by splitting nutritional substrates into $\mathrm{CO}_{2}$ and water, can be performed. In short, an energy-rich proton gradient is created over the inner mitochondrial membrane (IMM) by pumping protons into the intermembrane space using energy derived from splitting substrates. This proton gradient then drives the production of ATP from ADP and inorganic phosphate. Oxidative phosphorylation was traditionally considered to be the only major function of mitochondria, but recent studies have revealed highly regulated roles for mitochondrial quality and quantity in key cellular regulatory pathways like apoptosis and autophagy (6).

As skeletal muscle mitochondrial quantity and as such oxidative capacity are clearly affected in chronic diseases, it is likely that mitochondrial homeostasis is altered in such a way that mitochondrial degradation, which is mainly mediated through mitophagy, is favored above mitochondrial biogenesis. The main focus has been on impaired skeletal muscle mitochondrial biogenesis in patients with chronic disease in the past decades (9-13), but remarkably, the role of mitochondrial breakdown remains understudied. This review aims to examine current evidence of skeletal muscle mitophagy in different chronic diseases, and to examine evidence linking common denominators of these diseases to increased mitophagy and the loss of skeletal muscle oxidative capacity. Finally, the putative role of skeletal muscle mitophagy in maintenance of muscle health will be discussed. 


\section{Mitochondrial dynamics}

According to popular belief, the mitochondrion used to be a prokaryote, which was engulfed by endocytosis in early eukaryotes millions of years ago. Today, mitochondria still contain their own DNA (mtDNA), which consists of 37 genes which are coding for several different mitochondrial proteins, protein subunits, or supporting molecules required for mitochondrial function. The remaining mitochondrial proteins and subunits are encoded by nuclear DNA. To ensure coordinated transcription of both the mIDNA and nuclear DNA, the peroxisome proliferator-activated receptor- $\gamma$ coactivator family acts as mitochondrial biogenesis master regulator (14).

Mitochondria are highly dynamic organelles, which are constantly changing in size and shape. These changes are mediated through mitochondrial fission and fusion events (6). Dynamin-related protein 1 is master regulator for mitochondrial fission, and works together with proteins like mitochondrial fission 1 and mitochondrial fission process 18 . Mitochondrial fusion is mainly regulated on the level of the IMM by optic atrophy 1 , and on the outer mitochondrial membrane (OMM) by mitofusin (MFN)-1, and MFN-2 (6). Due to the selective regulation of these processes, mitochondria in one cell can be highly heterogeneous in size, function, and morphology, and mtDNA copy number.

One of the functions of fusion is to 'dilute' damaged mtDNA or proteins in large mitochondrial networks to enable mitochondrial damage repair. However, because of their exposure to high concentrations of reactive oxygen species (ROS), mitochondria are highly susceptible for protein and mtDNA damage, which can easily become very extensive (15). To ensure overall mitochondrial health, the mitochondrial network is able to direct damaged mtDNA and proteins into a specific mitochondrial area, which can then be separated from the main mitochondrial network via mitochondrial fission and broken down via mitophagy (6).

There are three different levels for clearance of mitochondrial content. The first is the mitochondrial ubiquitin-proteasome system, where specific (damaged) proteins are targeted for destruction without influencing the function of the mitochondria (6). Second, there is the formation and off-budding of small mitochondrial-derived vesicles, which are subsequently cleared via lysosomal breakdown (6). Finally, mitochondrial autophagy (mitophagy) clears complete or large pieces of mitochondria, and is therefore an important regulator of mitochondrial quantity. 


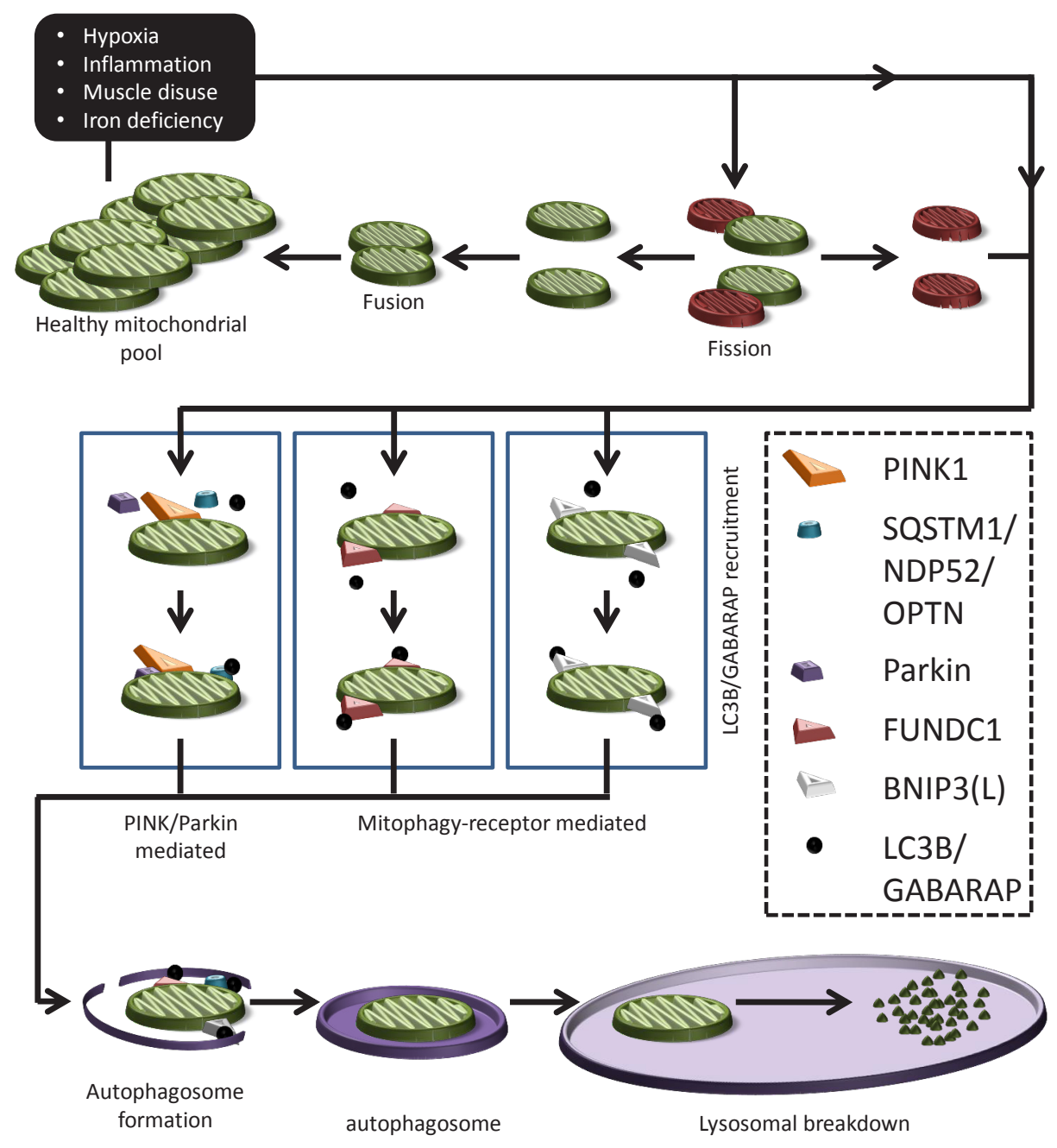

Figure 1: Proposed mitophagy-initiation pathway in skeletal muscle of patients with chronic disease. Skeletal muscle is exposed to hypoxia, inflammation, muscle disuse, and/or iron deficiency, resulting in either mitochondrial damage or simply the need to adjust or recycle healthy mitochondrial content. Mitochondrial fission is initiated depending on mitochondrial size and the extent of mitochondrial damage, leaving either parts of or the complete mitochondria for breakdown. Subsequently, the mitochondria are primed for autophagosomal engulfment by LC3B/GABARAP recruitment. LC3B/GABARAP either binds the activated receptor proteins BNIP3, BNIP3L, or FUNDC1, or in case of dysfunctional mitochondria to PINK1-recruited Parkin/SQSTM1, NDFP52, or OPTN. LC3B/GABARAP mediates autophagosomal formation around the mitochondrion and subsequent lysosomal breakdown. 


\section{Mitophagy}

Mitophagy initiation is generally divided in, but is not exclusive to, two main pathways which are extensively reviewed in other studies $(14,16,17,18)$. Although these pathways are often described separately, crosstalk between these pathways has been shown. Figure 1 depicts a short summary of the different mitophagy pathways, and their most important players, as described below.

The first pathway is the receptor-based mitophagy pathway. This pathway selectively targets specific mitochondria by post-translational activation of OMM bound mitophagy receptor proteins. By activation of these receptor proteins (i.e., BCL2/adenovirus $\mathrm{E} 1 \mathrm{~B} 19 \mathrm{kDa}$ protein-interacting protein (BNIP)3, BNIP3L, FUN14 domain-containing protein 1 (FUNDC1)), their binding to an autophagosomal-specific protein of the microtubule-associated protein 1A/1B-light chain 3 (LC3) or $\gamma$-aminobutyric acid receptor-associated protein (GABARAP) family is facilitated, and subsequent autophagosomal engulfment and lysosomal degradation is initiated. The selection of mitochondria which are targeted for mitophagy via this pathway is based on the activation of these receptors and not directly related to mitochondrial health $(14,17,18)$.

The second mitophagy pathway is the phosphatase and tensin homologueinduced kinase 1 (PINK1)/E3 ubiquitin-protein ligase parkin (Parkin) pathway, which is specific for mitochondria with a dysfunctional membrane potential (16). In healthy mitochondria, PINK1 is continuously imported to the IMM space where it is broken down by mitochondrial proteases and the proteasome. When the mitochondrial membrane potential is lost however, PINK1 import is compromised and it accumulates on the OMM of the mitochondria. There it attracts and activates several proteins of which Parkin has been described best, but nuclear dot protein $52 \mathrm{kDa}$ (NDP52) and optineurin (OPTN) were found to be recruited independently of Parkin as well $(19,20)$. Activated Parkin subsequently ubiquitinates several OMM bound proteins, which can be used as docking station for sequestosome 1 (SQSTM1). SQSTM1, OPTN, and NDP52 are autophagy receptors which, like the mitophagy receptor proteins discussed above, are able to bind a member of the autophagosomal-specific protein family, leading to subsequent autophagosomal engulfment and lysosomal degradation $(14,16,17)$. 


\section{Evidence for skeletal muscle mitophagy in chronic disease}

To date, only a selected number of studies have investigated mitophagy in the skeletal muscle of patients with COPD, T2D, or CHF. Guo et al. (21) reported increased presence of the mitophagy-related proteins BNIP3 and PARK2 together with an upregulation of general autophagy in vastus lateralis muscle of COPD patients. However, whether this was indeed associated with altered mitochondrial content or oxidative capacity was not assessed in this study (21). In contrast, Kruse et al. (22) reported no differences in autophagy-related and mitophagy-related markers in the vastus lateralis muscle of type 2 diabetic study participants compared with healthy volunteers. However, as no markers for oxidative capacity or mitochondrial quantity were reported, oxidative capacity loss in this study population remains undetermined (22). As mentioned earlier, fission is often associated with mitophagy and could therefore be regarded as an indirect indicator of mitophagy. Average mitochondrial size was shown to be decreased in vastus lateralis muscle of patients with CHF (23) or T2D (24), which is suggestive of a mitochondrial fission/fusion balance change toward mitochondrial fission. However, decreased mitochondrial size was not found in COPD patients (25). Bach et al. (26) reported decreased MFN-2 gene expression in the skeletal muscle of T2D patients, whereas Garnier et al. (27) reported no significant differences in skeletal muscle MFN-2 gene expression in CHF patients compared with sedentary controls. Despite this unchanged gene expression, Molina et al. (4) reported that the skeletal muscle protein expression of MFN-2 was decreased in CHF patients compared with sedentary controls. Taken together, these data point toward enhanced muscle mitophagy in chronic diseases.

\section{Triggers of mitophagy in chronic disease}

Disease-related factors already known for their ability to impair muscle oxidative capacity and hence are putative mitophagy initiators include hypoxia, systemic inflammation, muscle inactivity, and iron deficiency $(1,3,5,28,29)$.

\section{Hypoxia}

As recently reviewed, hypoxia was consistently shown to result in decreased skeletal muscle mitochondrial density and oxidative capacity in both rodents and humans (30). Moreover, increased BNIP3-mediated mitophagy was found during hypoxia in several nonmuscle in-vitro cell lines (31), and increases in BNIP3 gene expression were reported in both murine skeletal muscle tissue and in-vitro cells 
exposed to hypoxia (32). This transcriptional initiation of mitophagy receptors BNIP3 and BNIP3L by hypoxia is the result of increased ROS production, and subsequent hypoxia-inducible factor-1 $\alpha$ (HIF-1 $\alpha$ ) stabilization $(33,34)$.

In addition to BNIP3(L)-mediated mitophagy, activation of mitophagy receptor FUNDC1 was reported during hypoxia. Interestingly, hypoxia does not seem to affect FUNDC1 transcription, which is in contrast with hypoxia-induced BNIP3(L) transcription, but activates FUNDC1 on a post-transcriptional level by phosphorylation or inhibition of dephosphorylation $(35,36,37)$. Whether crosstalk between the BNIP3(L) and FUNDC1 pathways is present during hypoxia-induced mitophagy remains to be studied.

Although it is thought that human skeletal muscle is quite resistant to chronic hypoxia-induced HIF-1 $\alpha$ stabilization, HIF- $1 \alpha$-specific muscle adaptions have been reported (38). A study comparing gene expression of BNIP3, GABARAPL, LC3, and Beclin in the vastus lateralis of hypoxemic COPD patients to normoxemic COPD patients reported no differences (39), but this study only reported gene expression and only for a limited number of mitophagyrelated proteins. To date, it remains to be determined whether chronic disease-induced skeletal muscle hypoxia results in mitophagy, and whether this mitophagy targets healthy or dysfunctional mitochondria.

\section{Inflammation}

Systemic inflammation results in a decreased skeletal muscle oxidative capacity in mice (40), and skeletal muscle inflammatory signaling is associated with decreased skeletal muscle oxidative phenotype in chronic disease (41). Like with hypoxia, systemic inflammation is associated with increased ROS production in skeletal muscle mitochondria (42). However, whether inflammation induced ROS production also results in HIF-1 $\alpha$ stabilization and subsequent BNIP3L-mediated mitophagy remains undetermined. ROS also initiates the NF-KB signaling pathway (42), which was indeed shown to causally induce LC3B and GABARAPL1 gene expression in the skeletal muscle of a systemic inflammation mouse model (43). However, increased GABARAPL1 gene expression was still found upon NF-KB signaling inhibition, and increased BNIP3 gene expression was found to be completely NF-KB independent in this model (43). It is therefore assumable that BNIP3-mediated mitophagy is present during systemic inflammation, but the regulatory mechanisms still need to be unraveled.

Interestingly, inflammation-induced mitophagy was found to be regulated through the PINK1/Parkin pathway as well. It has been shown that increased ROS production leads to opening of the mitochondrial permeability transition 
pore, a large IMM and OMM spanning channel that upon opening disrupts the mitochondrial membrane potential and leads to mitochondrial dysfunction (44). These dysfunctional mitochondria stabilize PINK1 and subsequently recruit Parkin, making this a pathway that selectively targets dysfunctional mitochondria. Alongside this pathway however, NF-KB was found to be able to stabilize cytosolic PINK1 as well, and is therefore able to target healthy mitochondria for nonselective mitophagy (45). In inflammation subjected cardiac muscle, PINK1/Parkin-mediated mitophagy was indeed found to be increased, but Parkin was found to be dispensable for mitochondrial clearance in this model (46). Parkin independecy was found in other models as well, where NDP52 and OPTN were identified as alternative receptorproteins $(19,20)$. In conclusion, it is feasible that mitophagy is enhanced in skeletal muscle of patients suffering from (low-grade) systemic inflammation, and is hence underlying loss of oxidative capacity.

\section{Muscle disuse}

Physical inactivity and muscle disuse are associated with decreased muscle oxidative capacity (47). In addition, increased mitophagy was shown in different immobilization studies. Kang et al. (48) recently showed increased Parkin, decreased BNIP3, and increased general autophagy-related protein expression in unloaded tibialis anterior muscle of mice, and Vainshtein et al. (49) found an increase in BNIP3L, Parkin, LC3BII, and SQSTM1 protein expression in the tibialis anterior after denervation. Although no literature is available on mitophagy in human immobilized muscle, the occurrence of mitophagy is feasible as Gram et al. (50) found increased ROS production in human immobilized skeletal muscle. In contradiction, Drummond et al. (51) found that inactive frail women had both decreased BNIP3 and Parkin gene expression in their vastus lateralis, which the authors explained as a possible adapted homeostasis to low muscle mass and physical function. Taken together, it is likely that muscle disuse is an important early trigger of mitophagy, probably as part of a normal physiological adaptation to adjust mitochondrial content to the reduced energy demand associated with lower physical activity levels. Whether this mitophagy targets healthy unused mitochondria, dysfunctional mitochondria, or both is unknown.

\section{Iron deficiency}

Iron deficiency has been linked to impaired exercise capacity and decreased mitochondrial activity in animal models (28). In addition, iron deficiency in $\mathrm{CHF}$ patients is correlated with impaired exercise capacity (52). Moreover, iron therapy resulted in increased physical performance in iron-deficient CHF patients (28), suggestive of improved oxidative capacity. Although no studies have been performed to link iron deficiency to increased mitophagy in the skeletal muscle, 
iron deficiency was shown to induce BNIP3(L) and PINK1/Parkin-mediated mitophagy in C. elegans and several nonmuscular human cell-lines $(53,54)$. As mitochondria contain high amounts of iron, it could be speculated that mitophagy is required during episodes of iron deficiency to mobilize iron for other essential iron-dependent processes.

\section{Mitophagy: Friend or foe?}

The question arises whether skeletal muscle mitophagy is a good or a bad process. On the one hand, mitophagy may serve to clear healthy mitochondria in case they are either redundant or contain essential components required elsewhere. On the other hand, mitophagy is essential for mitochondrial quality control and attenuated mitophagy could result in extensive mitochondrial damage, dysfunction, and even cell death.

Although the knowledge about skeletal muscle mitophagy is still limited, many studies have been performed in heart muscle, which have been extensively reviewed (55). Indeed, it has been well established that both insufficient and exacerbated mitophagy in the heart leads to cardiomyopathy, and should, therefore, be tightly regulated (55). Such a safeguarding function for mitophagy has also been found in human vascular smooth muscle cells (56). Interventions to combat the loss of muscle oxidative capacity targeted directly at mitophagysignaling should, therefore, be approached with the highest caution.

A reduction in mitophagy will not only rescue healthy mitochondria but result in an increased number of dysfunctional mitochondria as well, which could aggravate the decrease in muscular health even more. Therefore, it is instrumental to obtain additional knowledge concerning the disease-related mitophagy inducing triggers and the mitophagy signaling pathways. Moreover, it could be argued that interventions should be aimed at the prevention of mitochondrial damage, mitochondrial disuse, and nutrient shortage, rather than the pharmacological inhibition of mitophagy pathways itself. 


\section{Conclusion}

The balance between mitophagy and mitochondrial biogenesis is a tightly regulated process, which can be affected by many different factors. We identified several factors which are shared between different chronic diseases, each able to initiate mitophagy independently. We propose that these factors work together as a complex combination of synergistic mitophagy triggers (Fig. 1), resulting in the loss of skeletal muscle oxidative capacity in patients with chronic disease. To prevent the loss of oxidative capacity, it could be argued that therapies should focus on these mitophagy-inducing triggers rather than mitophagy itself, to prevent aggravation of mitochondrial damage and subsequent muscle disorder. 


\section{References}

1. Maltais F, Decramer M, Casaburi R, et al. An official American Thoracic Society/ European Respiratory Society statement: update on limb muscle dysfunction in chronic obstructive pulmonary disease. Am J Respir Crit Care Med 2014; 189:e15-e62.

2. Stanford KI, Goodyear LJ. Exercise and type 2 diabetes: molecular mechanisms regulating glucose uptake in skeletal muscle. Adv Physiol Educ 2014; 38:308-314.

3. Putti R, Migliaccio V, Sica R, Lionetti L. Skeletal muscle mitochondrial bioenergetics and morphology in high fat diet induced obesity and insulin resistance: focus on dietary fat source. Front Physiol 2015; 6:426.

4. Molina AJ, Bharadwaj MS, Van Horn C, et al. Skeletal muscle mitochondrial content, oxidative capacity, and Mfn2 expression are reduced in older patients with heart failure and preserved ejection fraction and are related to exercise intolerance. JACC Heart Fail 2016; 4:636-645.

5. Kinugawa $S$, Takada $S$, Matsushima $S$, et al. Skeletal muscle abnormalities in heart failure. Int Heart J 2015; 56:475-484.

6. Romanello V, Sandri M. Mitochondrial quality control and muscle mass maintenance. Front Physiol 2015; 6:422.

7. van de Bool C, Gosker HR, van den Borst B, et al. Muscle quality is more impaired in sarcopenic patients with chronic obstructive pulmonary disease. J Am Med Dir Assoc 2016; 17:415-420.

8. Huffman KM, Koves TR, Hubal MJ, et al. Metabolite signatures of exercise training in human skeletal muscle relate to mitochondrial remodelling and cardiometabolic fitness. Diabetologia 2014; 57:2282-2295.

9. Konokhova $\mathrm{Y}$, Spendiff S, Jagoe RT, et al. Failed upregulation of TFAM protein and mitochondrial DNA in oxidatively deficient fibers of chronic obstructive pulmonary disease locomotor muscle. Skelet Muscle 2016; 6:10.

10. Remels $A H$, Schrauwen P, Broekhuizen R, et al. Peroxisome proliferator-activated receptor expression is reduced in skeletal muscle in COPD. Eur Respir J 2007; 30:245-252.

11. van den Borst B, Slot IG, Hellwig VA, et al. Loss of quadriceps muscle oxidative phenotype and decreased endurance in patients with mild-to-moderate COPD. J Appl Physiol 2013; 114:1319-1328.

12. Mootha VK, Lindgren CM, Eriksson KF, et al. PGC-1alpha-responsive genes involved in oxidative phosphorylation are coordinately downregulated in human diabetes. Nat Genet 2003; 34:267-273.

13. Patti ME, Butte AJ, Crunkhorn S, et al. Coordinated reduction of genes of oxidative metabolism in humans with insulin resistance and diabetes: potential role of PGC1 and NRF1. Proc Natl Acad Sci U S A 2003; 100:8466-8471.

14. Stotland A, Gottlieb RA. Mitochondrial quality control: easy come, easy go.Biochim Biophys Acta 2015; 1853:2802-2811.

15. Czarny P, Pawlowska E, Bialkowska-Warzecha J, et al. Autophagy in DNA damage response. Int J Mol Sci 2015; 16:2641-2662.

16. Matsuda N. Phospho-ubiquitin: upending the PINK-Parkin-ubiquitin cascade. J Biochem 2016; 159:379-385. 
17. Wei H, Liu L, Chen Q. Selective removal of mitochondria via mitophagy: distinct pathways for different mitochondrial stresses. Biochim Biophys Acta 2015; 1853:2784-2790.

18. Yamaguchi O, Murakawa T, Nishida K, Otsu K. Receptor-mediated mitophagy. J Mol Cell Cardiol 2016; 95:50-56.

19. Lazarou M, Sliter DA, Kane LA, et al. The ubiquitin kinase PINK1 recruits autophagy receptors to induce mitophagy. Nature 2015; 524:309-314.

20. Richter B, Sliter DA, Herhaus L, et al. Phosphorylation of OPTN by TBK1 enhances its binding to $\mathrm{Ub}$ chains and promotes selective autophagy of damaged mitochondria. Proc Natl Acad Sci U S A 2016; 113:4039-4044.

21. Guo Y, Gosker HR, Schols AM, et al. Autophagy in locomotor muscles of patients with chronic obstructive pulmonary disease. Am J Respir Crit Care Med 2013; 188:1313-1320.

22. Kruse R, Vind BF, Petersson SJ, et al. Markers of autophagy are adapted to hyperglycaemia in skeletal muscle in type 2 diabetes. Diabetologia 2015; 58:2087-2095.

23. Guzman Mentesana G, Baez AL, Lo Presti MS, et al. Functional and structural alterations of cardiac and skeletal muscle mitochondria in heart failure patients. Arch Med Res 2014; 45:237-246.

24. Kelley DE, He J, Menshikova EV, Ritov VB. Dysfunction of mitochondria in human skeletal muscle in type 2 diabetes. Diabetes 2002; 51:2944-2950.

25. Gosker HR, Hesselink MK, Duimel H, et al. Reduced mitochondrial density in the vastus lateralis muscle of patients with COPD. Eur Respir J 2007; 30:73-79.

26. Bach D, Naon D, Pich S, et al. Expression of Mfn2, the Charcot-Marie-Tooth neuropathy type 2A gene, in human skeletal muscle: effects of type 2 diabetes, obesity, weight loss, and the regulatory role of tumor necrosis factor alpha and interleukin-6. Diabetes 2005; 54:2685-2693.

27. Garnier A, Fortin D, Zoll J, et al. Coordinated changes in mitochondrial function and biogenesis in healthy and diseased human skeletal muscle. FASEB J 2005; 19:43-52.

28. Stugiewicz M, Tkaczyszyn M, Kasztura $M$, et al. The influence of iron deficiency on the functioning of skeletal muscles: experimental evidence and clinical implications. Eur J Heart Fail 2016; 18:762-773.

29. Vasquez A, Logomarsino JV. Anemia in chronic obstructive pulmonary disease and the potential role of iron deficiency. COPD 2016; 13:100-109.

30. Horscroft JA, Murray AJ. Skeletal muscle energy metabolism in environmental hypoxia: climbing towards consensus. Extrem Physiol Med 2014; 3:19.

31. Zhu Y, Massen S, Terenzio M, et al. Modulation of serines 17 and 24 in the LC3-interacting region of Bnip3 determines pro-survival mitophagy versus apoptosis. J Biol Chem 2013; 288:1099-1113.

32. de Theije CC, Langen RC, Lamers WH, et al. Distinct responses of protein turnover regulatory pathways in hypoxia- and semistarvation-induced muscle atrophy. Am J Physiol Lung Cell Mol Physiol 2013; 305:L82-L91.

33. Li L, Tan J, Miao Y, et al. ROS and autophagy: interactions and molecular regulatory mechanisms. Cell Mol Neurobiol 2015; 35:615-621.

34. Wu H, Chen Q. Hypoxia activation of mitophagy and its role in disease pathogenesis. Antioxid Redox Signal 2015; 22:1032-1046. 
35. Wu H, Xue D, Chen G, et al. The BCL2L1 and PGAM5 axis defines hypoxia-induced receptor-mediated mitophagy. Autophagy 2014; 10:1712-1725.

36. Wu W, Tian W, Hu Z, et al. ULK1 translocates to mitochondria and phosphorylates FUNDC1 to regulate mitophagy. EMBO Rep 2014; 15:566-575.

37. Tian W, Li W, Chen Y, et al. Phosphorylation of ULK1 by AMPK regulates translocation of ULK1 to mitochondria and mitophagy. FEBS Lett 2015;589:1847-1854.

38. Favier FB, Britto FA, Freyssenet DG, et al. HIF-1-driven skeletal muscle adaptations to chronic hypoxia: molecular insights into muscle physiology. Cell Mol Life Sci 2015; 72:4681-4696.

39. Costes F, Gosker H, Feasson L, et al. Impaired exercise training-induced muscle fiber hypertrophy and Akt/mTOR pathway activation in hypoxemic patients with COPD. J Appl Physiol (1985) 2015; 118:1040-1049.

40. Tanaka K, Tanaka M, Takegaki J, Fujino H. Preventive effects of electrical stimulation on inflammation-induced muscle mitochondrial dysfunction. Acta Histochem 2016; 118:464-470.

41. Remels AH, Gosker HR, Langen RC, et al. Classical NF-kB activation impairs skeletal muscle oxidative phenotype by reducing IKK-a expression. Biochim Biophys Acta 2014; 1842:175-185.

42. Ji LL. Redox signaling in skeletal muscle: role of aging and exercise. Adv Physiol Educ 2015; 39:352-359.

43. Langen RC, Haegens A, Vernooy JH, et al. NF-kB activation is required for the transition of pulmonary inflammation to muscle atrophy. Am J Respir Cell Mol Biol 2012; 47:288-297.

44. Zhang J, Wang X, Vikash V, et al. ROS and ROS-mediated cellular signaling. Oxid Med Cell Longev 2016; 2016:4350965.

45. Lim GG, Chua DS, Basil AH, et al. Cytosolic PTEN-induced putative kinase 1 is stabilized by the NF-kB pathway and promotes nonselective mitophagy. J Biol Chem 2015; 290:16882-16893.

46. Piquereau J, Godin R, Deschenes S, et al. Protective role of PARK2/Parkin in sepsis-induced cardiac contractile and mitochondrial dysfunction. Autophagy 2013; 9:1837-1851.

47. Gram M, Vigelso A, Yokota T, et al. Two weeks of one-leg immobilization decreases skeletal muscle respiratory capacity equally in young and elderly men. Exp Gerontol 2014; 58:269-278.

48. Kang C, Yeo D, Ji LL. Muscle immobilization activates mitophagy and disrupts mitochondrial dynamics in mice. Acta Physiol (Oxf) 2016. [Epub ahead of print]

49. Vainshtein A, Desjardins EM, Armani A, et al. PGC-1a modulates denervationinduced mitophagy in skeletal muscle. Skelet Muscle 2015; 5:9.

50. Gram M, Vigelso A, Yokota T, et al. Skeletal muscle mitochondrial H2 $\mathrm{O} 2$ emission increases with immobilization and decreases after aerobic training in young and older men. J Physiol 2015; 593:4011-4027.

51. Drummond MJ, Addison O, Brunker L, et al. Downregulation of E3 ubiquitin ligases and mitophagy-related genes in skeletal muscle of physically inactive, frail older women: a cross-sectional comparison. J Gerontol A Biol Sci Med Sci 2014; 69:1040-1048. 
52. Jankowska EA, Rozentryt P, Witkowska A, et al. Iron deficiency predicts impaired exercise capacity in patients with systolic chronic heart failure. J Card Fail 2011; 17:899-906.

53. Schiavi A, Maglioni S, Palikaras K, et al. Iron-starvation-induced mitophagy mediates lifespan extension upon mitochondrial stress in C. elegans. Curr Biol 2015; 25:1810-1822.

54. Allen GF, Toth R, James J, Ganley IG. Loss of iron triggers PINK1/Parkin-independent mitophagy. EMBO Rep 2013; 14:1127-1135.

55. Vasquez-Trincado C, Garcia-Carvajal I, Pennanen C, et al. Mitochondrial dynamics, mitophagy and cardiovascular disease. J Physiol 2016; 594:509-525.

56. Swiader A, Nahapetyan H, Faccini J, et al. Mitophagy acts as a safeguard mechanism against human vascular smooth muscle cell apoptosis induced by atherogenic lipids. Oncotarget 2016; 7:28821-28835. 
Molecular signalling towards mitochondrial breakdown is enhanced in skeletal muscle of patients with chronic obstructive pulmonary disease (COPD)

P.A. Leermakers, A.M.W.J. Schols, A.E.M. Kneppers, M.C.J.M. Kelders, C.C. de Theije, M. Lainscak, H.R. Gosker

Sci Rep. 2018 Oct 9;8(1):15007. 


\section{Abstract}

Loss of skeletal muscle mitochondrial oxidative capacity is well-established in patients with COPD, but the role of mitochondrial breakdown herein is largely unexplored. Currently, we studied if mitochondrial breakdown signalling is increased in skeletal muscle of COPD patients and associates with the loss of mitochondrial content, and whether it is affected in patients with iron deficiency (ID) or systemic inflammation. Therefore, mitophagy, autophagy, mitochondrial dynamics and content markers were analysed in vastus lateralis biopsies of COPD patients ( $\mathrm{N}=95, \mathrm{FEV}_{1} \%$ predicted: 39.0 [31.0-53.6]) and healthy controls ( $N=15, \mathrm{FEV}_{1} \%$ predicted: 112.8 [107.5-125.5]). Sub-analyses were performed on patients stratified by ID or C-reactive protein (CRP). Compared with controls, COPD patients had lower muscle mitochondrial content, higher BNIP3L and lower FUNDC1 protein, and higher Parkin protein and gene-expression. BNIP3L and Parkin protein levels inversely correlated with mtDNA/gDNA ratio and $\mathrm{FEV}_{1} \%$ predicted. ID-COPD patients had lower BNIP3L protein and higher BNIP3 gene-expression, while high CRP patients had higher BNIP3 and autophagyrelated protein levels. In conclusion, our data indicates that mitochondrial breakdown signalling is increased in skeletal muscle of COPD patients, and is related to disease severity and loss of mitochondrial content. Moreover, systemic inflammation is associated with higher BNIP3 and autophagy-related protein levels. 


\section{Introduction}

Skeletal muscle weakness contributes to poor clinical outcome and is associated with increased morbidity and mortality in patients with chronic obstructive pulmonary disease (COPD) $(1,2)$. Important drivers of muscle weakness are the loss of skeletal muscle mitochondrial oxidative capacity and content, which, together with a oxidative to glycolytic fibre-type shift, are well-established in COPD $(1,3,4)$. Moreover, skeletal muscle mitochondria isolated from COPD patients were found to be functionally impaired and produce more reactive oxygen species, which indicates reduced mitochondrial health (5).

Skeletal muscle oxidative capacity and mitochondrial quantity are mainly regulated by mitochondrial homeostasis, which is determined by the balance between mitochondrial biogenesis and mitochondrial breakdown (6). Several studies have previously reported impaired skeletal muscle mitochondrial biogenesis regulation in COPD patients $(3,7,8)$, but data on mitochondrial breakdown is limited and its relation to the loss of oxidative capacity unknown.

Although individual mitochondrial proteins can be targeted for selective breakdown, bulk mitochondrial breakdown occurs via either autophagy-dependent (i.e. mitophagy) or autophagy-independent (i.e. mitochondria-derived vesicles (MDV)) lysosomal breakdown (9). During mitophagy, mitochondria, or parts thereof, are separated from the mitochondrial network, engulfed by an autophagosomal membrane and subsequently broken down by a lysosome. This pathway can roughly be divided in receptor-mediated mitophagy, activated by mitophagy-receptors like BCL2/Adenovirus E1B 19 kDa proteininteracting protein 3 (BNIP3), BNIP3-like (BNIP3L), and FUN14 domain-containing protein 1 (FUNDC1) (10-12), and PINK1/Parkin-mediated mitophagy, initiated by stabilization and activation of PTEN-induced putative kinase 1 (PINK1) and Parkin (13-15). During MDV formation, which also requires PINK1 and Parkin, only a small portion of the mitochondrion is isolated from the mitochondrial network and targeted for lysosomal breakdown independently of autophagy $(16,17)$.

Mitochondria are highly dynamic organelles, which are constantly changing in size and shape. These changes are mediated through mitochondrial fission and fusion events. Mitochondrial fission has been suggested to be play an important role in isolating mitochondria from the mitochondrial network, priming them for mitophagy (9). Fission is regulated by master-regulator Dynamin 1 Like (DNM1L), which is also known as Dynamin-related protein 1 (DRP1), and proteins like Mitochondrial fission 1 (FIS1), while fusion is mainly regulated on by Optic atrophy 1 (OPA1), and proteins from the Mitofusin (MFN) family (9). 
Interestingly, Guo et al. previously showed some indications of increased mitophagy in skeletal muscle of COPD patients (18), but the relation between the mitochondrial breakdown and mitochondrial content in the skeletal muscle of COPD patients remains unstudied.

Although it is unlikely that the COPD-related lung pathology directly regulates the activation of these mitochondrial breakdown-pathways in skeletal muscle, these patients usually suffer from several extra-pulmonary manifestations which may be implicated in the development of skeletal muscle- and mitochondrial dysfunction, like cigarette smoke exposure, muscle inactivity, iron deficiency, and systemic inflammation (19-21). Interestingly, both iron deficiency and systemic inflammation, which are commonly present in COPD patients, are not only linked to decreased exercise performance or oxidative capacity in chronic diseases $(22,23)$, but to induction of mitophagy in non-skeletal muscle models as well (24-26). Therefore, it is likely that these manifestations have negative impact on the skeletal muscle mitochondrial content in COPD patients by inducing the initiation of mitochondrial breakdown.

In the current study, we hypothesised that mitochondrial breakdown signalling is increased in skeletal muscle of COPD patients and is associated with the loss of mitochondrial content. In addition, we hypothesized that iron deficiency (ID) or enhanced systemic inflammation result in enhanced mitochondrial breakdown signalling in these patients. We tested these hypotheses by assessing differences in skeletal muscle expression of molecular markers of mitochondrial breakdown (i.e. BNIP3, BNIP3L, FUNDC1, PINK1, PARK2), general autophagy (i.e. LC3B, GABRARAPL1, SQSTM1, OPTN, CALCOCO2), and mitochondrial dynamics (i.e. DNM1L, FIS1, OPA1, MFN1) between COPD patients and controls, and studied if the mitochondrial breakdown markers correlated with markers for mitochondrial content (i.e. NDUFB8, SDHB, UQCR2, MT-COI, ATP5A, $\mathrm{mtDNA} / \mathrm{gDNA}$ ratio). Moreover, we studied differences in expression of markers in these panels between COPD patients stratified by either iron deficiency, or the systemic inflammation marker C-reactive protein (CRP) plasma levels.

\section{Methods}

\section{Ethical approval}

Vastus lateralis biopsies of COPD patients and healthy controls, previously gathered in two different cohort studies (Maastricht cohort; www.trialregister. nl: NTR1402, and Golnik cohort; www.clinicaltrials.gov: NCT02550808) were analysed post-hoc. The Maastricht cohort study was approved by the Maastricht 
University Medical Centre+ ethical review board (Maastricht, the Netherlands), and the Golnik cohort study was approved by the Slovenian National Medical Ethics Committee (Ljubljana, Slovenia). In total the studied population consisted of 95 COPD patients with clinically stable disease (i.e. free from exacerbations in the 4 weeks prior to the study) and 15 healthy age-matched controls. Study protocols were in accordance with the latest version of the Helsinki Declaration, approved by the respective ethics committees, and written informed consent was obtained from all subjects prior to the start. Spirometry was performed according to the European Respiratory Society guidelines. Data including smoking status, calculated $\mathrm{FEV}_{1}$ percentage predicted ( $\mathrm{FEV}_{1} \%$ predicted) (27) and disease severity, based on Global Initiative for Chronic Obstructive Lung Disease (GOLD) stage (28), was available for all patients.

Muscle biopsies were obtained under local anaesthetic under resting conditions using the needle biopsy technique, snap-frozen in liquid nitrogen, and stored at $-80^{\circ} \mathrm{C}$ until molecular analyses as described previously $(3,29)$.

\section{mRNA extraction and quantification}

mRNA extraction and quantification was performed as described previously (30) with primer details shown in Supplementary Table 1. In short, tissue was homogenized with the Beat-Beater in presence of TRI-reagent (Sigma-Aldrich, Zwijndrecht, the Netherlands), and RNA was isolated by TRI-reagent/Chloroform extraction and subsequently precipitated from the aqueous phase using glycogen-containing isopropanol. RNA concentrations were measured spectrophotometrically using a Nanodrop UV-Vis spectrophotometer (Thermo Scientific, Landsmeer, The Netherlands). 400 ng RNA was diluted in nuclease free $\mathrm{H}_{2} \mathrm{O}$ and reverse transcribed to cDNA using the Tetro cDNA synthesis kit (Bioline, Waddinxveen, The Netherlands) according to the manufacturer's instructions. qPCR reactions contained Sensimix SYBR \& ROX (Bioline, Waddinxveen, The Netherlands) and primer mix and were run in a 384 well white opaque plate on a LightCycler 480 system (Roche, Almere, The Netherlands). Melting curves were analysed to verify specificity of the amplification, and relative quantity of the targets was assessed by LinRegPCR software (v2014.8.1). Three reference genes (RPLPO, B2M, and PPIA) were used to calculate a GeNorm correction factor, which was used to normalize expression of the target genes. Specific sample measurements were excluded when individual PCR efficiency was deviating from average PCR efficiency. 


\section{DNA extraction and quantification}

DNA was purified from the organic fraction, generated with the TRI-reagent/Chloroform RNA extraction, according to manufacturer's protocol (Sigma-Aldrich, Zwijndrecht, the Netherlands), with the modification of centrifugation speed to $12,000 \mathrm{~g}$. DNA was pelleted, and dissolved in TE buffer (Sigma-Aldrich, Zwijndrecht, The Netherlands). qPCR was performed as described previously (30) with mitochondrial (COX-II) and genomic (RPL13A) specific primers (Supplementary Table 1). Data is presented as ratio of the relative copy number of mtDNA over gDNA.

\section{Western Blot}

Western Blotting and quantification was performed as described previously (30), with primary antibodies listed in Supplementary Table 2. In short, tissue was homogenized in $600 \mu \mathrm{l}$ of Immunoprecipitation lysis buffer (50mM Tris, $150 \mathrm{mM} \mathrm{NaCl}, 10 \%$ glycerol, $0.5 \%$ Nonidet P40, protease and phosphatase inhibitors (Roche, Almere, The Netherlands)) with a Polytron homogenizer (Kinematica, Eschbach, Germany) and centrifuged at $14,000 \mathrm{~g}$ at $4^{\circ} \mathrm{C}$ for $30 \mathrm{~min}$. $5 \mu \mathrm{g}$ of either unheated or heated $\left(5 \mathrm{~min}\right.$ at $\left.100^{\circ} \mathrm{C}\right)$ protein in $1 \mathrm{x}$ Laemmli sample buffer (0.25M Tris-HCL ph6.8; 8\% (w/v) SDS; 40\% (v/v) glycerol; 0.4M DTT and $0.02 \%(w / v)$ Bromophenol Blue) was separated on a Criterion XT Precast 4-12 or $12 \%$ Bis-Tris gel (Bio-Rad Laboratories B.V., Veenendaal, The Netherlands) in XT MOPS or MES running buffer (Bio-Rad Laboratories B.V., Veenendaal, The Netherlands) by gel electrophoresis. Proteins were transferred to a nitrocellulose membrane (Bio-Rad Laboratories B.V., Veenendaal, The Netherlands) by electroblotting at $100 \mathrm{~V}$ for 60 min in transfer buffer (25 mM Tris, 192 mM Glycine, $20 \%$ (vol/vol) methanol).

Membranes were stained with $0.2 \%$ Ponceaus in $1 \%$ predicted acetic acid (Sigma-Aldrich, Zwijndrecht, The Netherlands) and imaged with the Amersham ${ }^{\mathrm{Tm}}$ Imager 600 (GE Healthcare Life Sciences, Eindhoven, The Netherlands), to correct for protein loading. Subsequently, the membranes were blocked at room temperature (RT) in Tween20 Tris-buffered saline (TBST; $20 \mathrm{mM}$ Tris, $137 \mathrm{mM} \mathrm{NaCl}, 0.1 \%$ predicted (vol/vol) Tween20, $\mathrm{pH} 7.6$ ) containing 3\% (w/v) nonfat dry milk (Campina, Zaltbommel, The Netherlands), washed in TBST, and incubated overnight with primary antibody diluted 1:500-1:1,000 in TBST with $3 \%(\mathrm{w} / \mathrm{v} /) \mathrm{BSA}$ or non-fat dry milk at $4^{\circ} \mathrm{C}$. Membranes were washed, incubated with a HRP-conjugated secondary antibody (Vector Laboratories, Amsterdam, The Netherlands), diluted 1:10,000 in 3\% (w/v) non-fat dry milk in TBST, washed, incubated in Supersignal West PICO or FEMTO Chemiluminescent Substrate (Thermo Scientific, Landsmeer, The Netherlands), and imaged using the Amersham ${ }^{\mathrm{TM}}$ Imager 600. Images were quantified with Image Quant 
software (GE Healthcare Life Sciences, Eindhoven, The Netherlands). Samples and protein loading reference lanes were randomly distributed within and between multiple gels (Supplementary Figure 3). All samples derive from the same experiment and gels/blots were processed in parallel. Target protein quantity was corrected for total protein content, and between-gel differences, and presented as a fold change relative to the control group. Measurements were excluded when signs of protein deterioration were found. All original data can be found in the online supplement available on https://doi.org/10.1038/s41598-018-33471-2.

\section{Blood measurements}

Blood samples were obtained from all patients at the start of the study protocol. Serum ferritin levels, transferrin saturation, iron levels, and haemoglobin levels were measured in the patients from the Golnik cohort. Subjects were considered iron deficient (ID-COPD) when either absolute iron deficiency (ferritin $<100 \mathrm{ng} / \mathrm{ml}$ ) or functional iron deficiency (ferritin 100-300 ng/ml and transferrin saturation $<20 \%$ ) was present (22). Other patients were defined as non-iron deficient (NID-COPD) patients.

Serum CRP was determined as a marker for systemic inflammation with the CardioPhase $^{\circledR}$ high-sensitive CRP kit (Siemens Healthcare Diagnostics Inc., Newark, USA) with a lower limit of detection of $0.18 \mathrm{mg} / \mathrm{L}$, in the COPD patients from the Maastricht cohort (31). Subjects were considered to have low CRP when $C R P \leq 3.0 \mathrm{mg} / \mathrm{L}$ and high CRP when CRP $>3.0 \mathrm{mg} / \mathrm{L}$.

\section{Statistics}

Variables were tested for normality using Shapiro-Wilk Test. Patient characteristics were presented in tables as percentage, mean (SD), or median [interquartile range] based on distribution of data. All molecular markers were, independently of normal distribution, presented graphically in bar charts as mean + SEM for coherence and clarity purposes. Statistical relevance of observed groupdifferences was tested with chi-squared test for categorized variables, Mann-Whitney U test for non-normal distributed variables, or independent t-test for normal distributed variables. The exact number of cases tested per variable is depicted in the tables for patient characteristics, and in Supplementary Table 1 and 2 for molecular markers. Correlations were tested with Spearman's rho analysis's in the combined cohorts $(\mathrm{N}=89-94)$. All statistical tests were computed using IBM SPSS Statistics software (version 22.0, IBM Corp., Armonk, NY, USA). 


\section{Results}

COPD patients have altered levels of mitochondrial breakdown related markers compared with healthy controls. Patients had mild-to-severe COPD and were similar to control subjects with respect to age, sex, and body composition. In addition, patients had a different distribution of smoking status with a higher proportion of current- and ex-smokers compared with healthy controls. As expected, $M Y H 7$ gene-expression, a surrogate marker for type I fibre proportion, was lower in the patients (Table 1).

Table 1: Characteristics of study population

\begin{tabular}{|c|c|c|}
\hline & Control & COPD \\
\hline & $(\mathrm{N}=15)$ & $(\mathrm{N}=95)$ \\
\hline \multicolumn{3}{|l|}{ Demographics } \\
\hline 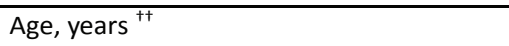 & $65.1(6.0)$ & $65.0(7.8)$ \\
\hline Sex, \% male ${ }^{+}$ & 60.0 & 66.3 \\
\hline $\begin{array}{l}\text { Smoking status } \\
\text { (never, ex, current), } \%^{+}\end{array}$ & $47,47,6$ & $0,28,72 * * *$ \\
\hline \multicolumn{3}{|l|}{ Lung function } \\
\hline $\mathrm{FEV}_{1}, \%$ predicted $^{+++}$ & $112.8[107.5-125.5]$ & $39.0[31.0-53.6] * * *$ \\
\hline $\mathrm{FEV}_{1} / \mathrm{FVC}, \%^{+++}$ & $73.3[69.3-77.8]$ & $35.6[28.1-46.4] * * *$ \\
\hline GOLD stage $(1,2,3,4), \%^{+}$ & & $3,23,51,23$ \\
\hline \multicolumn{3}{|l|}{ Body composition } \\
\hline $\mathrm{BMI}, \mathrm{kg} / \mathrm{cm}^{2+++}$ & $23.9[23.0-26.1]$ & $25.6[22.2-27.3]$ \\
\hline \multicolumn{3}{|l|}{ Muscle composition } \\
\hline MYH7 mRNA expression, Arbitrary Units ${ }^{+++}$ & $1.043[0.602-1.265]$ & $0.405[0.166-0.693] * * *$ \\
\hline
\end{tabular}

Abbreviations: $\mathrm{BMI}$, body mass index; $\mathrm{FEV}_{1}$, forced expiratory volume in 1 second; $\mathrm{FVC}$, forced vital capacity; GOLD, Global Initiative for Chronic Obstructive Lung Disease. †Data presented as percentage and statistical differences were calculated with chi-squared test; $\dagger+$ data presented as mean (SD) and statistical differences were calculated with independent samples t-test; $+\dagger+$ data presented as median [interquartile range] and statistical differences were calculated with Mann-Whitney $U$ test. Statistical significance is depicted ${ }^{* * *} p<0.001$.

BNIP3L protein levels were higher in the vastus lateralis of COPD patients compared with controls (figure $1 A$ ). No differences were found for BNIP3 and BNIP3L gene-expression (Figure 1B). Moreover, FUNDC1 protein levels were found to be lower in the patients, while there was no corresponding change in FUNDC1 gene-expression (Figure 1A-B).

Microtubule associated protein 1A/1B-light chain 3 B (LC3B) and $\gamma$-aminobutiric acid receptor-associated protein-like 1 (GABARAPL1) are autophagosomal membrane-based proteins with high affinity for respectively binding 
BNIP3/FUNDC1 and BNIP3L, which are needed for mitochondrial autophagosomal engulfment. Higher protein levels of the premature, cytoplasmicbased (LC3BI), but not of the mature, autophagosomal membrane-based (LC3BII), form of LC3B were found, resulting in a lower LC3BII/LC3BI protein ratio in COPD patients (Figure $1 \mathrm{C}$ ). Both protein levels and gene-expression of GABARAPL1, and gene-expression of $\angle C 3 B$ were not different between groups (Figure 1C-D).

A

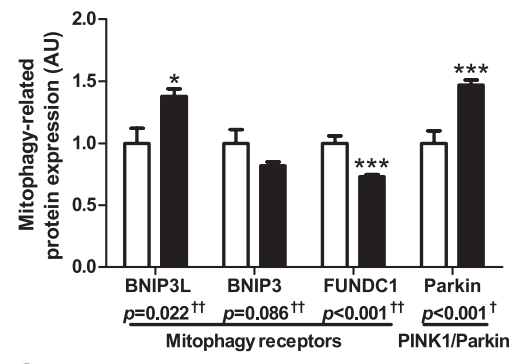

C
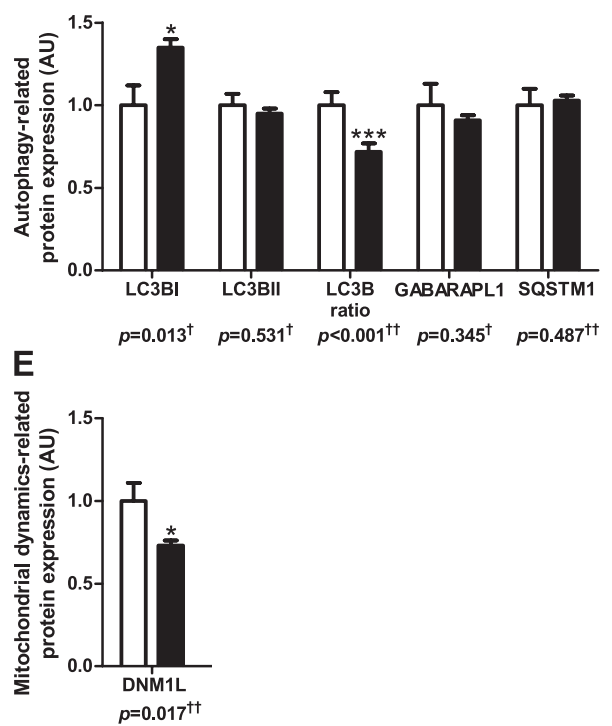

B

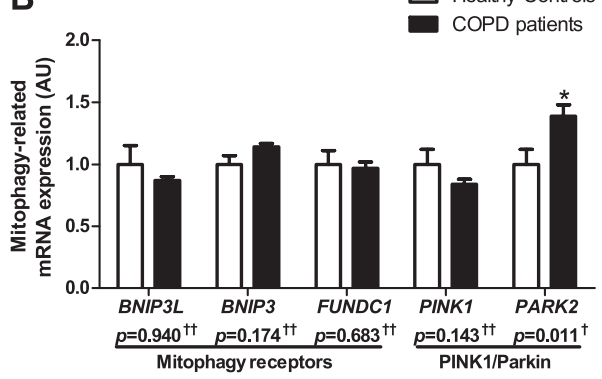

D
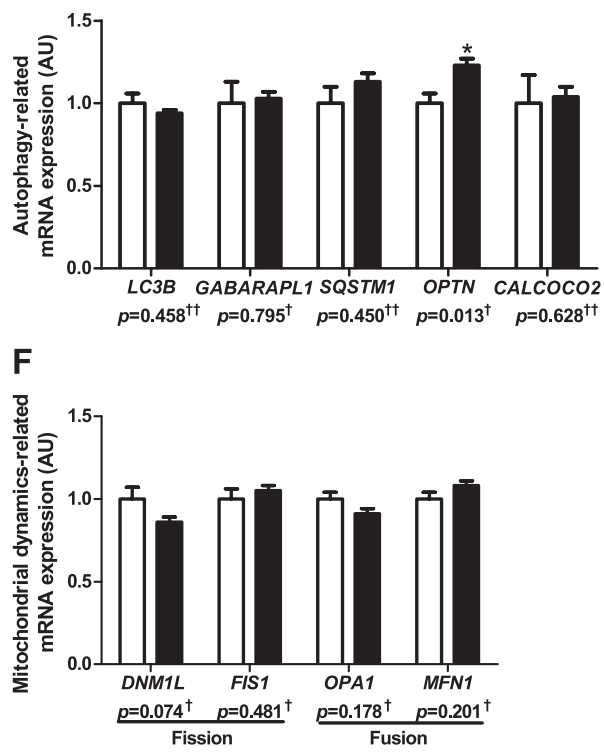

Figure 1: Protein and mRNA expression of markers related to mitophagy and autophagy in $m$. vastus lateralis of COPD patients and representative controls. Mitophagy-associated protein levels (A) and mRNA expression (B) are depicted. Autophagy-associated protein levels (C) and mRNA expression (D) are depicted. Mitochondrial dynamics-associated protein levels (E) and mRNA expression (F) are depicted. White bars represent healthy controls and black bars represent COPD patients. All samples derive from the same experiment and gels/blots were processed in parallel. Data presented as mean \pm SEM. Variables had tnormal or +tnon-normal distribution and $p$-value of $\dagger$ parametric or $+\dagger$ non-parametric test and significant differences are depicted ${ }^{*} p<0.05$, $* * * p<0.001$. 
Parkin protein- and gene-expression was higher in patients compared with controls (Figure 1A-B), whilegene-expression of PINK1 was not different (figure 1B). Although no differences were found in protein levels or gene-expression for the downstream autophagy-receptors sequestosome 1 (SQSTM1) and Calcium Binding And Coiled-Coil Domain 2 (CALCOCO2) (Figure 1C-D), gene-expression of autophagy-receptor Optineurin (OPTN) was higher in COPD patients (Figure 1D). Protein levels of mitochondrial fission master regulator DNM1L were lower in vastus lateralis of COPD patients compared with controls (Figure 1E). Geneexpression of mitochondrial fission or fusion markers was not different between the groups (Figure 1F).

COPD patients have a lower mitochondrial quantity compared with healthy controls, and both mitochondrial quantity and $\mathrm{FEV}_{1} \%$ predicted correlate moderately with the mitochondrial breakdown markers. All tested subunits of oxidative phosphorylation (OXPHOS) complexes as well as the mtDNA/gDNA ratio were lower in the vastus lateralis of COPD patients compared with controls (Figure 2A-B).
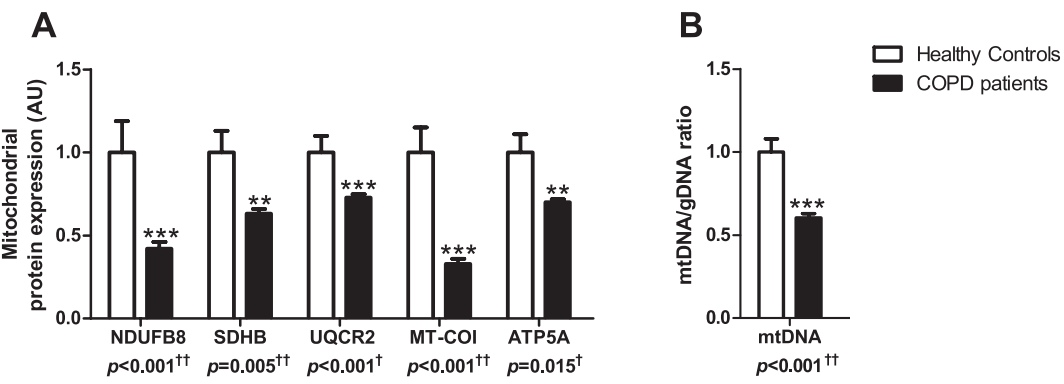

Figure 2: Protein levels of mitochondrial content markers and mtDNA/gDNA ratio in $m$. vastus lateralis of COPD patients and representative controls. Protein levels of different mitochondrial OXPHOS subunits are depicted (A). NDUFB8 as a subunit of OXPHOS complex I, SDHB of complex II, UQCRC2 of complex III, MT-COI of complex IV, and ATP5A of complex V. mtDNA/gDNA ratio is depicted (B). White bars represent healthy controls and black bars represent COPD patients. All samples derive from the same experiment and gels/blots were processed in parallel. Data presented as mean \pm SEM. Variables had tnormal or + tnon-normal distribution and $p$-value of + parametric or + tnon-parametric test and significant differences are depicted ${ }^{*} p<0.05, * * p<0.01, * * * p<0.001$.

Since the mtDNA/gDNA ratio is widely accepted as a solid overall marker of mitochondrial content, we studied its correlation to the protein levels of BNIP3L $(\rho=-0.288, p=0.006)$, BNIP3 $(\rho=0.183, p=0.083)$, FUNDC1 $(\rho=0.259, p=0.014)$, and Parkin ( $\rho=-0.209, \mathrm{p}=0.049$ ) (Supplementary Figure $1 \mathrm{~A}-\mathrm{D})$ ). The mtDNA/gDNA ratio correlated with $\mathrm{FEV}_{1} \%$ predicted $(\rho=0.407, p<0.001)$ as well, suggesting a link between disease severity and mitochondrial content in COPD. Therefore, we also studied the correlation between $\mathrm{FEV}_{1} \%$ predicted and BNIP3L ( $\left.\rho=-0.308, p=0.003\right)$, 
BNIP3 $(\rho=-0.003, p=0.976)$, FUNDC1 $(\rho=0.461, p<0.001)$ and Parkin $(\rho=-0.361$, $p<0.001$ ) (Supplementary Figure 2A-D).

\section{Iron deficient COPD patients have altered levels of BNIP3- and BNIP3L-mediated} mitophagy markers compared with non-iron deficient COPD patients. To test whether muscle mitophagy is affected by iron deficiency in COPD patients, patients from the Golnik cohort were subdivided in ID-COPD and NID-COPD. Patients with ID-COPD were not different with respect to age, sex, smoking status, BMI, and plasma haemoglobin levels when compared with NID-COPD. However, they had a better lung function, and higher $\mathrm{MYH7}$ gene-expression (Table 2).

Table 2: Characteristics of the subset of COPD patients stratified by iron deficiency

\begin{tabular}{|c|c|c|}
\hline & non-iron deficient COPD & iron deficient COPD \\
\hline & $(\mathrm{N}=44)$ & $(N=19)$ \\
\hline \multicolumn{3}{|l|}{ Demographics } \\
\hline 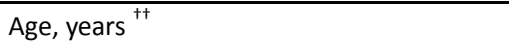 & $63.8(8.4)$ & $67.3(8.8)$ \\
\hline Sex, $\%$ male $^{\dagger}$ & 75.0 & 55.6 \\
\hline $\begin{array}{l}\text { Smoking status } \\
\text { (never, ex, current), } \%^{+}\end{array}$ & $0,12,88$ & $0,6,94$ \\
\hline \multicolumn{3}{|l|}{ Lung function } \\
\hline $\mathrm{FEV}_{1}, \%$ predicted $^{++\dagger}$ & $32.5[25.3-40.3]$ & $40.0[34.0-60.0] *$ \\
\hline $\mathrm{FEV}_{1} / \mathrm{FVC}, \%^{+++}$ & $29.8[26.5-37.8]$ & $37.1[33.0-43.5] *$ \\
\hline $\operatorname{GOLD}(1,2,3,4), \%^{+}$ & $0,7,57,36$ & $0,32,53,16^{*}$ \\
\hline \multicolumn{3}{|l|}{ Body composition } \\
\hline $\mathrm{BMI}, \mathrm{kg} / \mathrm{cm} 2^{++}$ & $24.7(3.8)$ & $25.9(4.9)$ \\
\hline \multicolumn{3}{|l|}{ Iron status } \\
\hline Serum iron $(\mu \mathrm{mol} / \mathrm{L})^{++}$ & $19.6(6.4)$ & $14.6(7.0) *$ \\
\hline 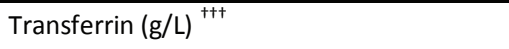 & $2.4[2.2-2.7]$ & $2.5[2.2-2.6]$ \\
\hline Transferrin Saturation $(\%)^{+++}$ & $31.0[24.0-40.0]$ & $22.0[17.0-26.0] * *$ \\
\hline Ferritin (ng/ml) ${ }^{++\dagger}$ & $222[158-294]$ & $78[58-116] * * *$ \\
\hline${\text { Haemoglobin }(\mathrm{g} / \mathrm{l})^{++}}^{++}$ & $147.0(13.0)$ & $142.1(8.9)$ \\
\hline \multicolumn{3}{|l|}{ Muscle composition } \\
\hline MYH7 mRNA expression, Arbitrary Units ${ }^{+++}$ & $0.179[0.128-0.479]$ & $0.517[0.323-0.837] *$ \\
\hline
\end{tabular}

Abbreviations: BMI, body mass index; FEV1, forced expiratory volume in 1 second; FVC, forced vital capacity; GOLD, Global Initiative for Chronic Obstructive Lung Disease. †Data presented as percentage and statistical differences were calculated with chi-squared test; +†data presented as mean (SD) and statistical differences were calculated with independent samples t-test; $+\dagger+$ data presented as median [interquartile range] and statistical differences were calculated with Mann-Whitney U test. Statistical significance is depicted ${ }^{*} p<0.05,{ }^{* *} p<0.01,{ }^{* * *} p<0.001$. 
BNIP3L protein levels were lower in the vastus lateralis of patients with ID-COPD compared with NID-COPD, while BNIP3 protein levels were not different (Figure 3A). Although no changes in BNIP3L gene-expression were found, BNIP3 gene-expression was higher in ID-COPD (Figure $3 B$ ). No differences were found for FUNDC1, PINK1, or Parkin (Figure 3A-B). Although LC3B gene-expression was higher in ID-COPD (Figure 3D), there was no change in protein levels of LC3B or LC3BII/LC3BI ratio (Figure $3 \mathrm{C}$ ). None of the autophagy receptors downstream of PINK1/Parkin-mediated mitophagy (i.e. SQSTM1, OPTN, CALCOCO2) showed

A
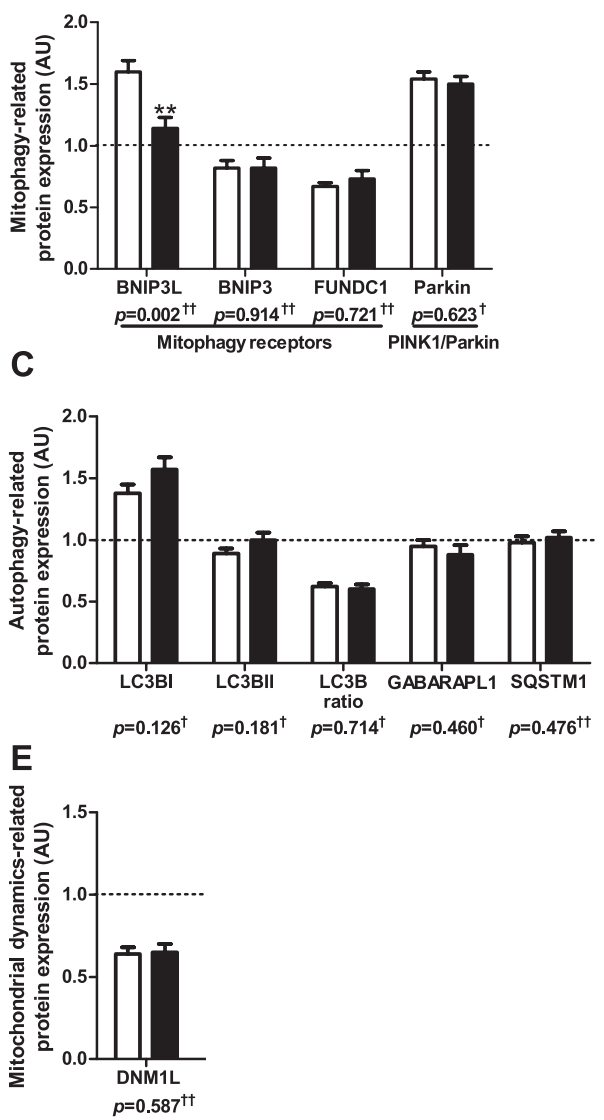
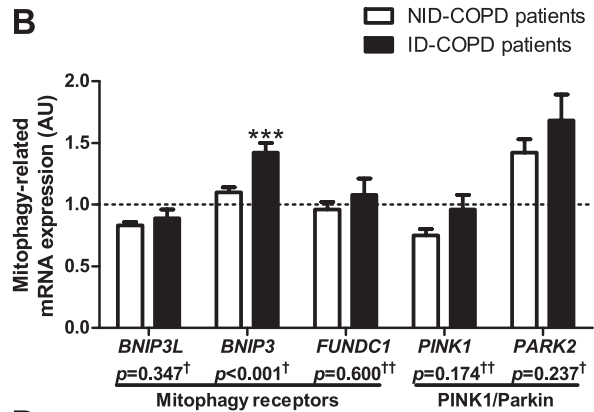

D
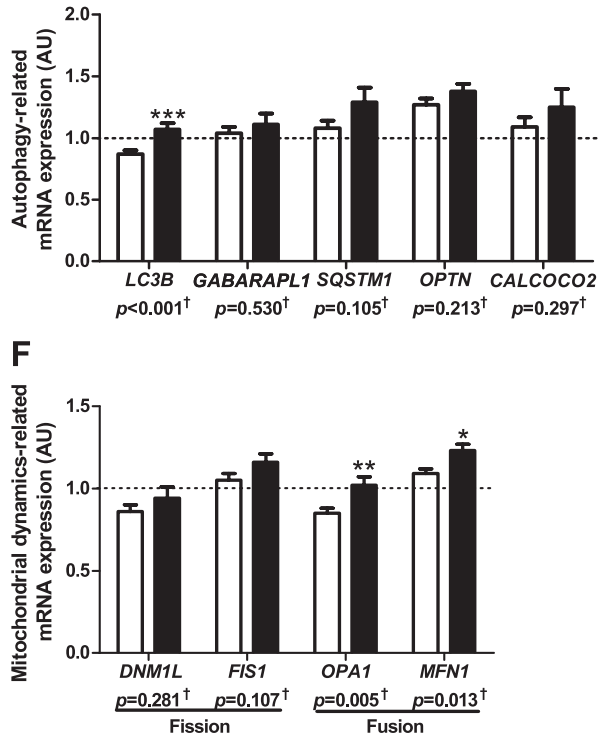

Figure 3: Protein and mRNA expression of markers related to mitophagy, autophagy, and mitochondrial dynamics in $m$. vastus lateralis of patients with NID-COPD and ID-COPD. Mitophagy-associated protein levels (A) and mRNA expression (B) are depicted. Autophagyassociated protein levels (C) and mRNA expression (D) are depicted. Mitochondrial dynamicsassociated protein levels $(\mathbf{E})$ and $\mathrm{mRNA}$ expression $(\mathbf{F})$ are depicted. White bars represent healthy controls and black bars represent COPD patients. All samples derive from the same experiment and gels/blots were processed in parallel. Data presented as mean \pm SEM. Dotted lines represent healthy controls. Variables had tnormal or t+non-normal distribution and $p$-value of tparametric or + tnon-parametric test and significant differences are depicted ${ }^{*} p<0.05, * * p<0.01,{ }^{* * *} p<0.001$. 
A

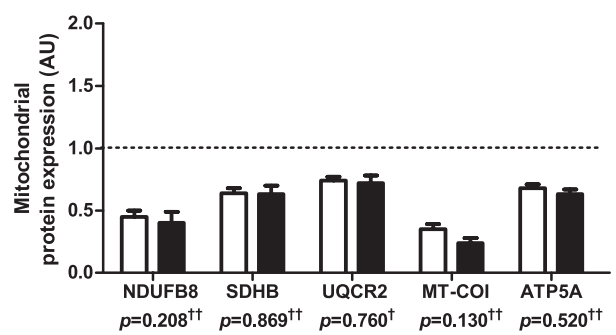

B

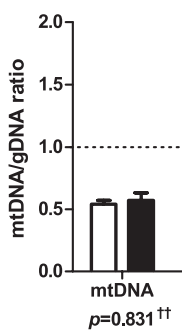

$\square$ NID-COPD patients

ID-COPD patients

Figure 4: Protein levels of mitochondrial content markers and mtDNA/gDNA ratio in $m$. vastus lateralis of patients with NID-COPD and ID-COPD. Protein levels of different mitochondrial OXPHOS subunits are depicted (A). NDUFB8 as a subunit of OXPHOS complex I, SDHB of complex II, UQCRC2 of complex III, MT-COI of complex IV, and ATP5A of complex V. mtDNA/gDNA ratio is depicted (B). White bars represent non-iron deficient COPD patients and black bars represent iron deficient COPD patients. All samples derive from the same experiment and gels/blots were processed in parallel. Data presented as mean \pm SEM. Dotted lines represent healthy controls. Variables had tnormal or t+non-normal distribution and p-value of tparametric or t+nonparametric test.

Table 3: Characteristics of the subset of COPD patients stratified by CRP levels

\begin{tabular}{|c|c|c|}
\hline & low CRP ( $\leq 3.0 \mathrm{mg} / \mathrm{L}$ ) & high CRP (> 3.0mg/L) \\
\hline & (N=19) & $(\mathrm{N}=9)$ \\
\hline \multicolumn{3}{|l|}{ Demographics } \\
\hline Age, years ${ }^{\text {}+}$ & $65.1(6.8)$ & $66.1(6.2)$ \\
\hline Sex, $\%$ male $^{\dagger}$ & 47.0 & 67.0 \\
\hline $\begin{array}{l}\text { Smoking status } \\
\text { (never, ex, current), } \%^{+}\end{array}$ & $0,74,26$ & $0,56,44$ \\
\hline \multicolumn{3}{|l|}{ Lung function } \\
\hline $\mathrm{FEV}_{1}, \%$ predicted $^{t+\dagger}$ & $62.6[46.0-74.1]$ & $47.9[44.1-58.4]$ \\
\hline $\mathrm{FEV}_{1} / \mathrm{FVC}, \%^{+++}$ & $45.6(13.3)$ & $43.3(6.4)$ \\
\hline $\operatorname{GOLD}(1,2,3,4), \%^{\dagger}$ & $16,53,32,0$ & $0,22,78,0$ \\
\hline \multicolumn{3}{|l|}{ Body composition } \\
\hline $\mathrm{BMI}, \mathrm{kg} / \mathrm{cm}^{2++}$ & $24.8(3.4)$ & $26.7(4.4)$ \\
\hline \multicolumn{3}{|l|}{ Systemic inflammation } \\
\hline $\operatorname{CRP}(\mathrm{mg} / \mathrm{L})^{t++}$ & $1.1[0.9-1.5]$ & $7.7[3.6-21.0] * * *$ \\
\hline \multicolumn{3}{|l|}{ Muscle composition } \\
\hline MYH7 mRNA expression, Arbitrary Units ${ }^{\text {t+ }}$ & $0.491[0.410-1.125]$ & $0.573[0.349-1.075]$ \\
\hline
\end{tabular}

Abbreviations: BMI, body mass index; CRP, C-reactive protein; FEV1, forced expiratory volume in 1 second; FVC, forced vital capacity; GOLD, Global Initiative for Chronic Obstructive Lung Disease. tData presented as percentage and statistical differences were calculated with chi-squared test; + data presented as mean (SD) and statistical differences were calculated with independent samples t-test; $+\dagger+$ data presented as median [interquartile range] and statistical differences were calculated with Mann-Whitney U test. Statistical significance is depicted ${ }^{* * *} p<0.001$. 
differential protein- or gene-expression (Figure 3C-D). Interestingly, geneexpression of the mitochondrial fusion markers OPA1 and MFN1 was higher in ID-COPD, while no differences were found for the mitochondrial fission-related DNM1L and FIS1 on either protein or mRNA level (Figure 3E-F). No differences in mitochondrial content were found in patients with ID-COPD compared with NID-COPD (Figure 4A-B).

A

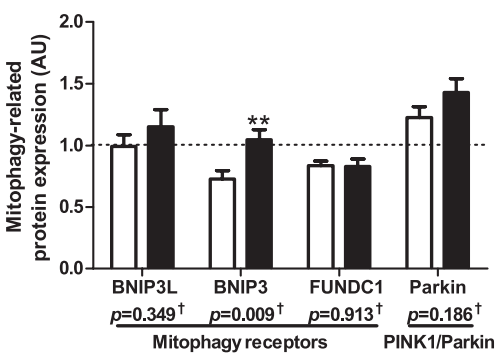

C

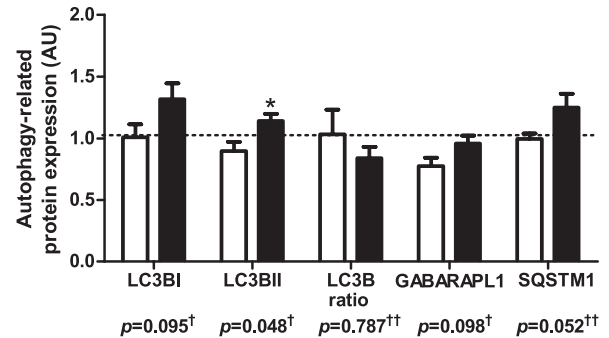

E

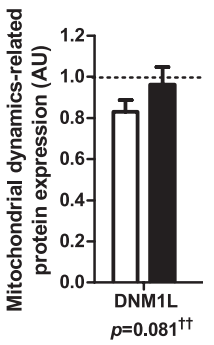

B

Patients with low CRP

Patients with high CRP

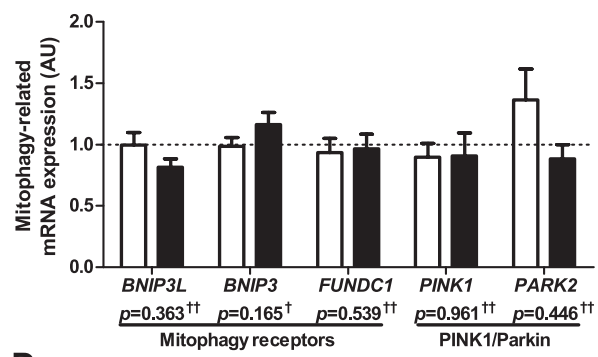

D

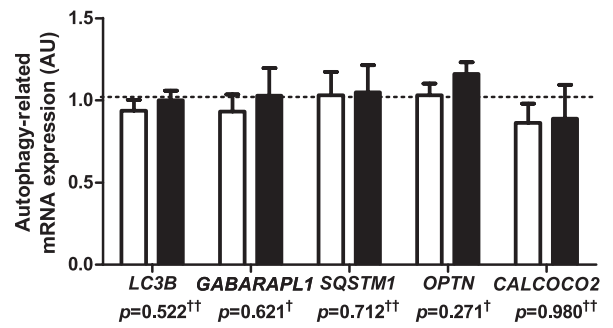

$\mathbf{F}$

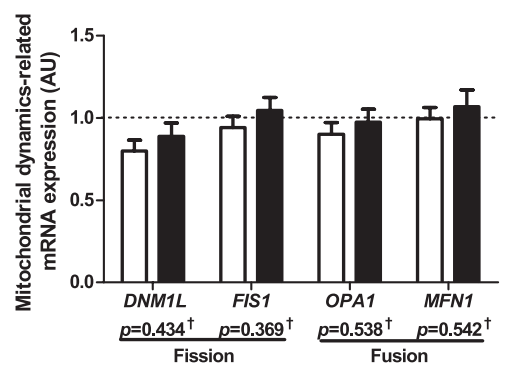

Figure 5: Protein and mRNA expression of markers related to mitophagy, autophagy, and mitochondrial dynamics in $m$. vastus lateralis of patients with low and high CRP. Mitophagyassociated protein levels (A) and mRNA expression (B) are depicted. Autophagy-associated protein levels (C) and mRNA expression (D) are depicted. Mitochondrial dynamics-associated protein levels $(\mathrm{E})$ and mRNA expression $(\mathbf{F})$ are depicted. White bars represent COPD patients with $\mathrm{CRP} \leq 3.0 \mathrm{mg} / \mathrm{L}$ and black bars represent COPD patients with CRP $>3 \mathrm{mg} / \mathrm{L}$. All samples derive from the same experiment and gels/blots were processed in parallel. Data presented as mean \pm SEM. Dotted lines represent healthy controls. Variables had tnormal or +tnon-normal distribution and $p$-value of $\dagger$ parametric or ${ }^{+t}$ non-parametric test and significant differences are depicted ${ }^{*} p<0.05$, $* * p<0.01$. 
Patients with high CRP have higher levels of BNIP3-mediated mitophagy markers compared with patients with low CRP. To test whether muscle mitophagy is affected by systemic inflammation in COPD patients, patients from the Maastricht cohort were divided in groups with high or low CRP. The groups were not different with respect to age, sex, smoking status, lung function, BMI, and MYH7 gene-expression (Table 3).

BNIP3 protein levels were higher in the vastus lateralis of patients with high CRP compared with patients with low CRP, while levels of other mitophagy-related proteins were not different (Figure 5A). Interestingly, while LC3BII was the only measured autophagy-related protein which had significantly higher levels in the patients with high CRP, many other autophagy-related proteins showed similar trends (i.e. LC3BI, GABARAPL1, and SQSTM1) (Figure 5C). Both mitophagyand autophagy-related gene-expression was not different between the groups (Figure 5B-D). No differences in any measured markers for mitochondrial dynamics were found (Figure $5 \mathrm{E}-\mathrm{F}$ ), or mitochondrial content were found in patients with high compared with patients with low CRP (Figure 6A-B). A schematic summary of all results is depicted in Figure 7.

A

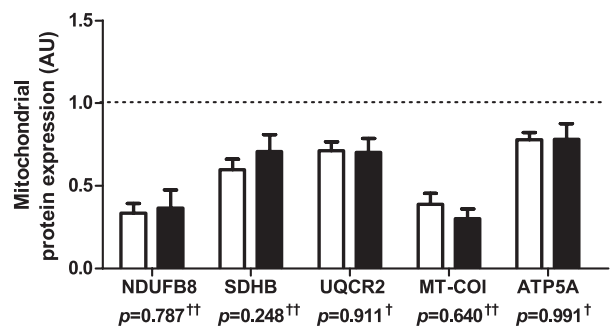

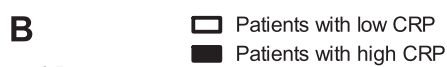

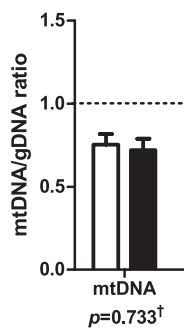

Figure 6: Protein levels of mitochondrial content markers and mtDNA/gDNA ratio in $m$. vastus lateralis of patients with low and high CRP. Protein levels of different mitochondrial OXPHOS subunits are depicted (A). NDUFB8 as a subunit of OXPHOS complex I, SDHB of complex II, UQCRC2 of complex III, MT-COI of complex IV, and ATP5A of complex V. mtDNA/gDNA ratio is depicted (B). White bars represent COPD patients with CRP $\leq 3.0 \mathrm{mg} / \mathrm{L}$ and black bars represent COPD patients with CRP $>3 \mathrm{mg} / \mathrm{L}$. All samples derive from the same experiment and gels/blots were processed in parallel. Data presented as mean \pm SEM. Dotted lines represent healthy controls. Variables had tnormal or t+non-normal distribution and $p$-value of tparametric or t+non-parametric test. 


\section{Discussion}

Our data indicates that mitochondrial breakdown is increased in quadriceps muscle of patients with COPD, illustrated by differential expression of both markers for receptor-mediated mitophagy and PINK1/Parkin-mediated mitochondrial breakdown. Secondly, we show that this expression pattern is associated with the decreased expression of mitochondrial content markers. Moreover, both BNIP3L and Parkin protein levels are inversely correlated with

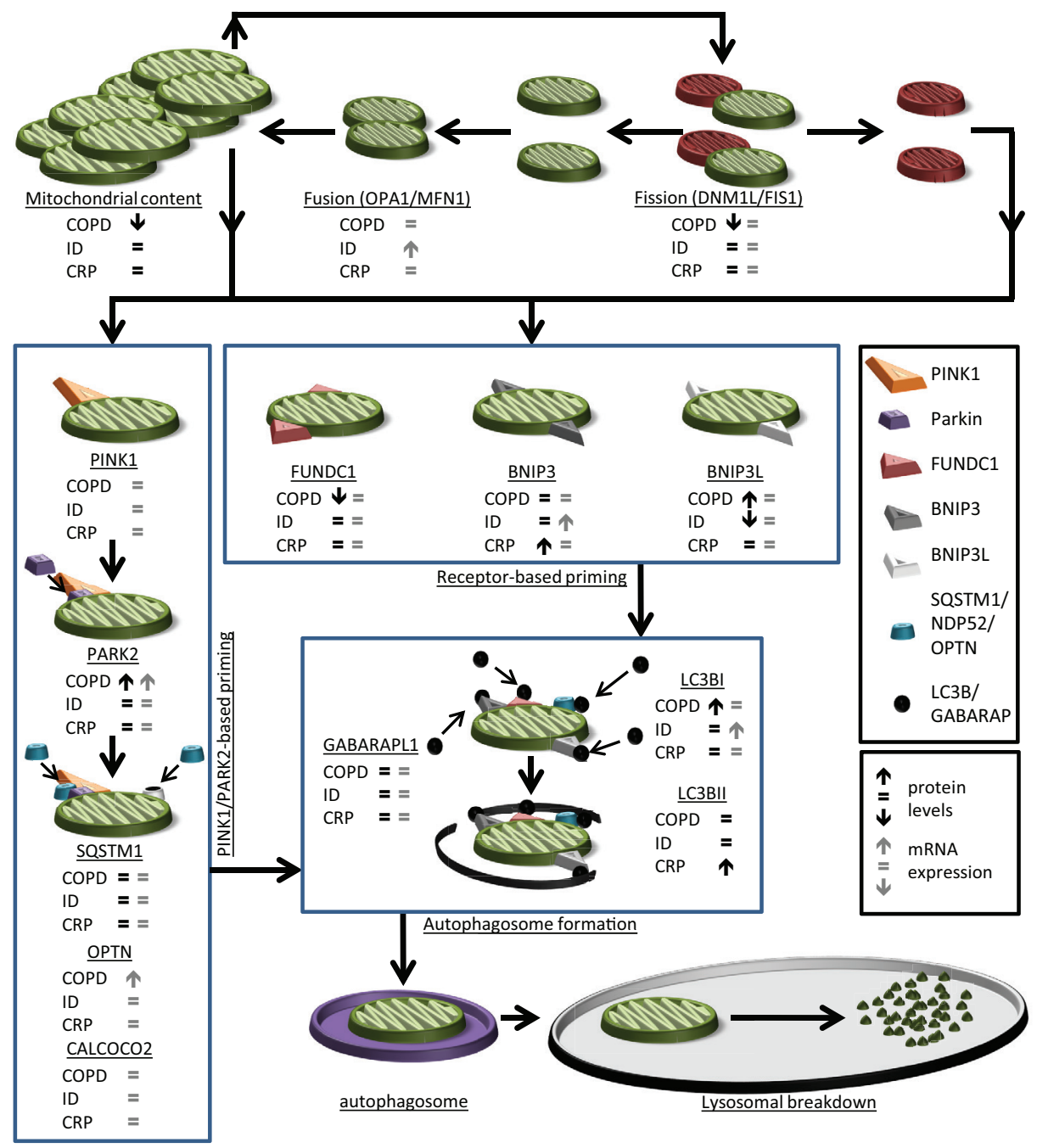

Figure 7: Schematic overview of differences in molecular signalling in the skeletal muscle of COPD compared with healthy control (COPD), ID-COPD compared with NID-COPD (ID) and COPD patients with high CRP compared with low CRP (CRP). 
mitochondrial content and disease severity within the group of patients. Lastly, we report differences in the expression pattern of markers for BNIP3 and BNIP3Lmediated mitophagy in COPD patients suffering from either iron deficiency or systemic inflammation. Although the interpretation of this differential expression remains difficult for ID, it suggests increased BNIP3-mediated mitophagy signalling in patients with systemic inflammation.

Receptor-mediated mitophagy is described as a pathway targeting mitochondria for mitophagy based on activation of specific receptors by upstream signalling (10). The currently reported higher protein levels of BNIP3L are a clear indication towards increased levels of this type of mitophagy in skeletal muscle of COPD patients. Moreover, BNIP3L was found to correlate inversely with the $\mathrm{mtDNA} / \mathrm{gDNA}$ ratio and $\mathrm{FEV}_{1} \%$ predicted, suggesting a gradual increase in the level of BNIP3L-mediated mitophagy with increasing disease severity and loss of mitochondrial content. However, we observed a trend towards lower levels of BNIP3, which were previously found to be higher in skeletal muscle of COPD patients (18). Since BNIP3 and BNIP3L are located on the outer mitochondrial membrane (32-34), an increase in BNIP3 protein expression might be masked by the lower mitochondrial content in our COPD population.

FUNDC1 is a relatively new hypoxia-regulated mitophagy-receptor of which the activity is dependent on its phosphorylation state rather than its transcriptional regulation. The activation of FUNDC1 therefore results in decreased protein levels as a direct result of increased mitophagy (35-37). Indeed, we found lower FUNDC1 protein levels in COPD patients, which were correlated to both the mtDNA/gDNA ratio as well as the $\mathrm{FEV}_{1} \%$ predicted, which could indicate disease severity dependent increased FUNDC1-mediated mitophagy in patients. However, FUNDC1 is a mitochondrial membrane-based protein as well, and lowerFUNDC1 levels might therefore be directly caused by the lower mitochondrial quantity in COPD patients. Although acute hypoxic stress could also result in a decrease of FUNDC1 protein independently of mitophagy (38), this is unlikely to be the case in our population due to the chronic and stable disease stage in which we obtained the biopsies.

PINK1 and Parkin have been described as major players in the mitophagy pathway, priming dysfunctional mitochondria for autophagosomal-lysosomal. Although the regulation and signal-transduction of PINK1 and Parkin is very complex, current literature suggest that PINK1 functions as an initiation protein and Parkin as a signal-amplifying protein in the priming of mitochondria for mitophagy $(13,15)$. In addition to mitophagy, both PINK1 and Parkin have been associated with MDV formation, an autophagy-independent mitochondrial 
breakdown pathway (16). We currently report higher gene-expression and protein levels of Parkin, suggesting increased Parkin-mediated mitochondrial breakdown in COPD patients. Taken together, these data, which are in concert with data from a previous study (18), indicate increased mitochondrial priming for lysosomal breakdown via either mitophagy or MDVs. Moreover, since Parkin was found to correlate inversely with mtDNA/gDNA ratio and the $\mathrm{FEV}_{1} \%$ predicted, the increase in Parkin protein levels appears to be more pronounced in both more severe patients and in patients with increased loss of mitochondrial content.

While DNM1L-mediated mitochondrial fission has been described as a prerequisite for mitophagy, MDV biogenesis could be performed independently of DNM1L $(9,16,39,40)$. Mitochondrial fission and fusion regulation is complex and highly integrated in muscle mass and quality control pathways (9). Although we did not quantify actual mitochondrial fission, the reported lower DNM1L protein-, and trend towards lower gene-expression, suggests that mitochondrial fission regulation is marginally decreased in COPD patients. Interestingly, a previous study reported decreased mitochondrial numbers while mitochondrial size was unaltered in the vastus lateralis of COPD patients (41), suggesting unaltered mitochondrial dynamics in these patients. Although we did not find a correlation between DNM1L protein levels and mtDNA/gDNA ratio in the current cohort of COPD patients $(\rho=0.108, p=0.312)$, the lower DNM1L levels could be part of a physiological response to maintain the amount of DNM1L per mitochondrion. Together with the current literature, our results are urging caution with interpretation of fission and fusion related signalling data.

The currently observed lower levels of mitochondrial content markers are in line with the well-established loss of oxidative capacity in skeletal muscle of patients with $\operatorname{COPD}(2,3,5,42)$, as well as with the well-described oxidative to glycolytic fibre-type shift, which is consistently observed in the peripheral skeletal muscle of COPD patients (4). Although we did not determine the percentage of oxidative type I fibres immunohistochemically in our study population, the lower expression of the MYH7 gene (a surrogate marker for type I fibres as it encodes the type I myosin heavy chain isoform (43)) in COPD is indicative of a decreased oxidative fibre type percentage which is in line with literature. Although we cannot exclude that differences in fibre type distribution influenced our findings, the fact that we found increased levels of mitophagy-related signalling despite the lower MYH7 gene expression, indicative of less mitochondria-rich type I fibres, in the patients suggests that enhanced mitochondrial breakdown may indeed be involved in the loss of muscle oxidative capacity in COPD. 
Interestingly, mtDNA/gDNA ratio was correlated, or showed a trend towards correlation, with BNIP3L, BNIP3, FUNDC1, and Parkin. Furthermore, mtDNA/gDNA ratio was also correlated with $\mathrm{FEV}_{1} \%$ predicted, which in turn was correlated with BNIP3L, FUNDC1 and Parkin. Together, these correlations show the highly interconnected nature of mitochondrial breakdown signalling, mitochondrial content, and disease severity.

Since it is unlikely that the COPD-related lung pathology directly regulates the activation of mitochondrial breakdown-pathways in skeletal muscle, we previously suggested a role for extra-pulmonary systemic manifestations (19). We therefore studied if iron deficiency and systemic inflammation are involved in the mitochondrial breakdown regulation in our COPD population.

Iron deficiency, often present but disregarded in COPD patients, has recently been shown to initiate mitophagy in non-skeletal muscle based models $(24,44)$. Unexpectedly, our ID-COPD patients had lower skeletal muscle BNIP3L protein levels than NID-COPD patients, resembling the levels of healthy controls, which could indicate decreased rather than increased mitophagy. However, ID-COPD patients had higher BNIP3 and LC3B gene- but not protein-expression, which could also indicate increased turnover of BNIP3 due to increased mitophagy. No differences were found in markers of either the FUNDC1- or PINK1/Parkinmediated mitochondrial breakdown in patients stratified by iron status. This is in line with a previous report showing iron depletion results in PINK1/Parkinindependent mitophagy in neuroblast cells (24). In conclusion, iron deficiency results in differential expression of BNIP3 and BNIP3L-related mitophagy markers, but does not seem to result in increased overall mitophagy in ID-COPD in our study population. Moreover, both the currently reported higher MYH7 gene-expression, indicative of more oxidative type I fibres, and better lung function in ID-COPD patients might affect our results, and therefore further research is needed to elucidate the exact impact of iron deficiency on mitophagy and the skeletal muscle oxidative phenotype.

The mitophagy-receptors BNIP3 and BNIP3L are known to be under transcriptional control of HIF-1 $\alpha$ (45), which is potentially regulated by several COPD-associated manifestations such as inflammation (46) and hypoxia (47). Indeed, patients with high CRP had higher skeletal muscle BNIP3 protein levels combined with higher levels of down-stream autophagy-related proteins. Although previous reports have indicated PINK1 and Parkin as important players in inflammation-mediated mitophagy both in vitro and in vivo, we report no differences in markers of either the FUNDC1- or PINK1/Parkin-mediated 
mitochondrial breakdown in patients with high $\operatorname{CRP}(25,26)$. However, these studies were performed with models exposed to profound inflammatory stimuli, almost certainly exceeding the low-grade systemic inflammation present in our patients, and might therefore surpass clinical relevance for our study population. In conclusion, we report higher BNIP3 and autophagy-related protein levels in patients with high systemic inflammation.

Besides iron deficiency and systemic inflammation, smoke exposure and increased muscle inactivity are among COPD-related extra-pulmonary manifestations which could potentially affect skeletal muscle mitochondrial impairments $(19,20)$. Indeed, our population of COPD patients had more current- and ex-smokers compared with the control population. Moreover, it is highly likely that these patients had lower physical activity levels as well (1), albeit that available literature in which physical activity levels have been linked to muscle biopsy analyses in COPD suggest that inactivity is a poor determinant of the loss of muscle oxidative phenotype in this disorder $(3,48,49)$. Although these manifestations were not the focus of the current study, it cannot be excluded that these factors contributed to the currently observed modulations in mitochondrial breakdown signalling in COPD patients and therefore these manifestations might be interesting targets for future research.

Although we show clear differences in mitochondrial breakdown-related markers, some limitations have to be addressed. First, we did not fully quantify the process of mitochondrial breakdown itself. This would require measuring actual mitophagy and/or MDV flux, for example by repeated measurements while blocking lysosomal breakdown (50). This is not feasible in human studies however, since lysosomal breakdown is required for long-term muscle maintenance. Moreover, continuous assessment of the studied parameters throughout disease progression would have been favourable, enabling determination of both the chronological order and the fluctuation of the studied processes. However, due to the invasive nature of muscle biopsies, and since they represent only one specific moment in time, we chose to study one time-point in a large heterogeneous group of patients.

In conclusion, this study shows altered expression of molecular markers for pathways of mitochondrial breakdown in skeletal muscle of COPD patients, which are related to disease severity and loss of mitochondrial quantity. Moreover, we report that both systemic inflammation and iron deficiency are associated with alterations in molecular markers for BNIP3 and BNIP3L-mediated mitophagy, and that while we report no coherent changes for ID-COPD, our results show higher 
BNIP3 and autophagy-related protein levels in patients with increased systemic inflammation. Taken together, the current data supports a potential role for mitochondrial breakdown underlying the loss of mitochondrial content in skeletal muscle of COPD patients, and identifies systemic inflammation as a possible mitophagy-inducing manifestation. This data is instrumental in understanding disturbed mitochondrial homeostasis in skeletal muscle of COPD, potentially leading towards new targets for maintaining or enhancing mitochondrial health. 


\section{References}

1. Maltais F, Decramer M, Casaburi R, Barreiro E, Burelle $Y$, Debigare R, et al. An official American Thoracic Society/European Respiratory Society statement: update on limb muscle dysfunction in chronic obstructive pulmonary disease. American journal of respiratory and critical care medicine. 2014 May 1;189(9): e15-62. PubMed PMID: 24787074. Pubmed Central PMCID: 4098112.

2. van den Borst B, Gosker HR, Schols AM. Central fat and peripheral muscle: partners in crime in chronic obstructive pulmonary disease. American journal of respiratory and critical care medicine. 2013 Jan 1;187(1):8-13. PubMed PMID: 23281350.

3. van den Borst B, Slot IG, Hellwig VA, Vosse BA, Kelders MC, Barreiro E, et al. Loss of quadriceps muscle oxidative phenotype and decreased endurance in patients with mild-to-moderate COPD. Journal of applied physiology. 2013 May;114(9):1319-28. PubMed PMID: 22815389.

4. Gosker HR, Wouters EF, van der Vusse GJ, Schols AM. Skeletal muscle dysfunction in chronic obstructive pulmonary disease and chronic heart failure: underlying mechanisms and therapy perspectives. The American journal of clinical nutrition. 2000 May;71(5):1033-47. PubMed PMID: 10799364.

5. Puente-Maestu L, Perez-Parra J, Godoy R, Moreno N, Tejedor A, GonzalezAragoneses $\mathrm{F}$, et al. Abnormal mitochondrial function in locomotor and respiratory muscles of COPD patients. The European respiratory journal. 2009 May;33(5): 1045-52. PubMed PMID: 19129279.

6. Palikaras K, Tavernarakis N. Mitochondrial homeostasis: The interplay between mitophagy and mitochondrial biogenesis. Experimental gerontology. 2014 Jan 28(56):182-8. PubMed PMID: 24486129.

7. Konokhova Y, Spendiff S, Jagoe RT, Aare S, Kapchinsky S, MacMillan NJ, et al. Failed upregulation of TFAM protein and mitochondrial DNA in oxidatively deficient fibers of chronic obstructive pulmonary disease locomotor muscle. Skeletal muscle. 2016;6:10. PubMed PMID: 26893822. Pubmed Central PMCID: 4758107.

8. Remels AH, Schrauwen P, Broekhuizen R, Willems J, Kersten S, Gosker HR, et al. Peroxisome proliferator-activated receptor expression is reduced in skeletal muscle in COPD. The European respiratory journal. 2007 Aug;30(2):245-52. PubMed PMID: 17459894.

9. Romanello V, Sandri M. Mitochondrial Quality Control and Muscle Mass Maintenance. Frontiers in physiology. 2015;6:422. PubMed PMID: 26793123. Pubmed Central PMCID: 4709858.

10. Yamaguchi O, Murakawa T, Nishida K, Otsu K. Receptor-mediated mitophagy. Journal of molecular and cellular cardiology. 2016 Mar 25(95):50-6. PubMed PMID: 27021519.

11. Wei H, Liu L, Chen Q. Selective removal of mitochondria via mitophagy: distinct pathways for different mitochondrial stresses. Biochimica et biophysica acta. 2015 Oct;1853(10 Pt B):2784-90. PubMed PMID: 25840011.

12. Stotland A, Gottlieb RA. Mitochondrial quality control: Easy come, easy go. Biochimica et biophysica acta. 2015 Oct;1853(10 Pt B):2802-11. PubMed PMID: 25596427. Pubmed Central PMCID: 4501896.

13. Matsuda N. Phospho-ubiquitin: upending the PINK-Parkin-ubiquitin cascade. Journal of biochemistry. 2016 Apr;159(4):379-85. PubMed PMID: 26839319. 
14. Lazarou M, Sliter DA, Kane LA, Sarraf SA, Wang C, Burman JL, et al. The ubiquitin kinase PINK1 recruits autophagy receptors to induce mitophagy. Nature. 2015 Aug 20;524(7565):309-14. PubMed PMID: 26266977.

15. Eiyama A, Okamoto K. PINK1/Parkin-mediated mitophagy in mammalian cells. Current opinion in cell biology. 2015 Apr;33:95-101. PubMed PMID: 25697963.

16. McLelland GL, Soubannier V, Chen CX, McBride HM, Fon EA. Parkin and PINK1 function in a vesicular trafficking pathway regulating mitochondrial quality control. The EMBO journal. 2014 Feb 18;33(4):282-95. PubMed PMID: 24446486. Pubmed Central PMCID: 3989637.

17. Soubannier V, Rippstein P, Kaufman BA, Shoubridge EA, McBride HM. Reconstitution of mitochondria derived vesicle formation demonstrates selective enrichment of oxidized cargo. PloS one. 2012;7(12):e52830. PubMed PMID: 23300790. Pubmed Central PMCID: 3530470.

18. Guo Y, Gosker HR, Schols AM, Kapchinsky S, Bourbeau J, Sandri M, et al. Autophagy in locomotor muscles of patients with chronic obstructive pulmonary disease. American journal of respiratory and critical care medicine. 2013 Dec 1;188(11): 1313-20. PubMed PMID: 24228729.

19. Leermakers PA, Gosker HR. Skeletal muscle mitophagy in chronic disease: implications for muscle oxidative capacity? Current opinion in clinical nutrition and metabolic care. 2016 Aug 16;19(6):427-33. PubMed PMID: 27537277.

20. Degens H, Gayan-Ramirez G, van Hees HW. Smoking-induced skeletal muscle dysfunction: from evidence to mechanisms. American journal of respiratory and critical care medicine. 2015 Mar 15;191(6):620-5. PubMed PMID: 25581779.

21. Bowen TS, Aakeroy L, Eisenkolb S, Kunth P, Bakkerud F, Wohlwend M, et al. Exercise Training Reverses Extrapulmonary Impairments in Smoke-exposed Mice. Medicine and science in sports and exercise. 2017 May;49(5):879-87. PubMed PMID: 28009790.

22. Barberan-Garcia A, Rodriguez DA, Blanco I, Gea J, Torralba Y, Arbillaga-Etxarri A, et al. Non-anaemic iron deficiency impairs response to pulmonary rehabilitation in COPD. Respirology. 2015 Oct;20(7):1089-95. PubMed PMID: 26148453.

23. Jankowska EA, Rozentryt P, Witkowska A, Nowak J, Hartmann O, Ponikowska B, et al. Iron deficiency predicts impaired exercise capacity in patients with systolic chronic heart failure. Journal of cardiac failure. 2011 Nov;17(11):899-906. PubMed PMID: 22041326.

24. Allen GF, Toth R, James J, Ganley IG. Loss of iron triggers PINK1/Parkin-independent mitophagy. EMBO reports. 2013 Dec;14(12):1127-35. PubMed PMID: 24176932.

25. Duan X, Tong J, Xu Q, Wu Y, Cai F, Li T, et al. Upregulation of human PINK1 gene expression by NFkappaB signalling. Molecular brain. 2014;7:57. PubMed PMID: 25108683. Pubmed Central PMCID: 4237968.

26. Piquereau J, Godin R, Deschenes S, Bessi VL, Mofarrahi M, Hussain SN, et al. Protective role of PARK2/Parkin in sepsis-induced cardiac contractile and mitochondrial dysfunction. Autophagy. 2013 Nov 1;9(11):1837-51. PubMed PMID: 24121678.

27. Miller MR, Crapo R, Hankinson J, Brusasco V, Burgos F, Casaburi R, et al. General considerations for lung function testing. The European respiratory journal. 2005 Jul;26(1):153-61. PubMed PMID: 15994402. 
28. Vestbo J, Hurd SS, Agusti AG, Jones PW, Vogelmeier C, Anzueto A, et al. Global strategy for the diagnosis, management, and prevention of chronic obstructive pulmonary disease: GOLD executive summary. American journal of respiratory and critical care medicine. 2013 Feb 15;187(4):347-65. PubMed PMID: 22878278.

29. Cebron Lipovec N, Schols AM, van den Borst B, Beijers RJ, Kosten T, Omersa D, et al. Sarcopenia in Advanced COPD Affects Cardiometabolic Risk Reduction by Short-Term High-intensity Pulmonary Rehabilitation. Journal of the American Medical Directors Association. 2016 Sep 1;17(9):814-20. PubMed PMID: 27321867.

30. Kneppers AEM, Langen RCJ, Gosker HR, Verdijk LB, Cebron Lipovec N, Leermakers PA, et al. Increased Myogenic and Protein Turnover Signaling in Skeletal Muscle of Chronic Obstructive Pulmonary Disease Patients With Sarcopenia. Journal of the American Medical Directors Association. 2017 Jul 01;18(7): 637 e1- e11. PubMed PMID: 28578881.

31. van den Borst B, Gosker HR, Wesseling G, de Jager W, Hellwig VA, Snepvangers FJ, et al. Low-grade adipose tissue inflammation in patients with mild-to-moderate chronic obstructive pulmonary disease. The American journal of clinical nutrition. 2011 Dec;94(6):1504-12. PubMed PMID: 22071714.

32. Chen G, Cizeau J, Vande Velde C, Park JH, Bozek G, Bolton J, et al. Nix and Nip3 form a subfamily of pro-apoptotic mitochondrial proteins. The Journal of biological chemistry. 1999 Jan 1;274(1):7-10. PubMed PMID: 9867803.

33. Imazu T, Shimizu S, Tagami S, Matsushima M, Nakamura Y, Miki T, et al. Bcl-2/E1B $19 \mathrm{kDa}$-interacting protein 3-like protein (Bnip3L) interacts with $\mathrm{bcl}-2 / \mathrm{Bcl}-\mathrm{xL}$ and induces apoptosis by altering mitochondrial membrane permeability. Oncogene. 1999 Aug 12;18(32):4523-9. PubMed PMID: 10467396.

34. Boyd JM, Malstrom S, Subramanian T, Venkatesh LK, Schaeper U, Elangovan B, et al. Adenovirus E1B $19 \mathrm{kDa}$ and $\mathrm{Bcl}-2$ proteins interact with a common set of cellular proteins. Cell. 1994 Oct 21;79(2):341-51. PubMed PMID: 7954800.

35. Lv M, Wang C, Li F, Peng J, Wen B, Gong Q, et al. Structural insights into the recognition of phosphorylated FUNDC1 by LC3B in mitophagy. Protein \& cell. 2017 Jan;8(1):25-38. PubMed PMID: 27757847. Pubmed Central PMCID: 5233613.

36. Liu L, Feng D, Chen G, Chen M, Zheng Q, Song P, et al. Mitochondrial outermembrane protein FUNDC1 mediates hypoxia-induced mitophagy in mammalian cells. Nature cell biology. 2012 Feb;14(2):177-85. PubMed PMID: 22267086.

37. Chen G, Han Z, Feng D, Chen Y, Chen L, Wu H, et al. A regulatory signaling loop comprising the PGAM5 phosphatase and CK2 controls receptor-mediated mitophagy. Molecular cell. 2014 May 8;54(3):362-77. PubMed PMID: 24746696.

38. Chen Z, Liu L, Cheng Q, Li Y, Wu H, Zhang W, et al. Mitochondrial E3 ligase MARCH5 regulates FUNDC1 to fine-tune hypoxic mitophagy. EMBO reports. 2017 Mar;18(3):495-509. PubMed PMID: 28104734. Pubmed Central PMCID: 5331199.

39. Gomes LC, Scorrano L. Mitochondrial morphology in mitophagy and macroautophagy. Biochimica et biophysica acta. 2013 Jan;1833(1):205-12. PubMed PMID: 22406072.

40. Neuspiel M, Schauss AC, Braschi E, Zunino R, Rippstein P, Rachubinski RA, et al. Cargo-selected transport from the mitochondria to peroxisomes is mediated by vesicular carriers. Current biology : CB. 2008 Jan 22;18(2):102-8. PubMed PMID: 18207745. 
41. Gosker HR, Hesselink MK, Duimel H, Ward KA, Schols AM. Reduced mitochondrial density in the vastus lateralis muscle of patients with COPD. The European respiratory journal. 2007 Jul;30(1):73-9. PubMed PMID: 17428811.

42. Taivassalo T, Hussain SN. Contribution of the Mitochondria to Locomotor Muscle Dysfunction in Patients With COPD. Chest. 2016 May;149(5):1302-12. PubMed PMID: 26836890.

43. Stuart CA, Stone WL, Howell ME, Brannon MF, Hall HK, Gibson AL, et al. Myosin content of individual human muscle fibers isolated by laser capture microdissection. Am J Physiol Cell Physiol. 2016 Mar 1;310(5):C381-9. PubMed PMID: 26676053. Pubmed Central PMCID: 4971827.

44. Schiavi A, Maglioni S, Palikaras K, Shaik A, Strappazzon F, Brinkmann V, et al. Iron-Starvation-Induced Mitophagy Mediates Lifespan Extension upon Mitochondrial Stress in C. elegans. Current biology : CB. 2015 Jul 20;25(14):1810-22. PubMed PMID: 26144971.

45. Sowter HM, Ratcliffe PJ, Watson P, Greenberg AH, Harris AL. HIF-1-dependent regulation of hypoxic induction of the cell death factors BNIP3 and NIX in human tumors. Cancer research. 2001 Sep 15;61(18):6669-73. PubMed PMID: 11559532.

46. Remels AH, Gosker HR, Verhees KJ, Langen RC, Schols AM. TNF-alpha-induced NF-kappaB activation stimulates skeletal muscle glycolytic metabolism through activation of HIF-1alpha. Endocrinology. 2015 May;156(5):1770-81. PubMed PMID: 25710281.

47. Slot IG, Schols AM, Vosse BA, Kelders MC, Gosker HR. Hypoxia differentially regulates muscle oxidative fiber type and metabolism in a HIF-1alpha-dependent manner. Cellular signalling. 2014 May 2;26(9):1837-45. PubMed PMID: 24794533.

48. Gifford JR, Trinity JD, Kwon OS, Layec G, Garten RS, Park SY, et al. Altered skeletal muscle mitochondrial phenotype in COPD: disease vs. disuse. J Appl Physiol (1985). 2018 Apr 1;124(4):1045-53. PubMed PMID: 29357496. Pubmed Central PMCID: 5972462.

49. Natanek SA, Gosker HR, Slot IG, Marsh GS, Hopkinson NS, Man WD, et al. Heterogeneity of quadriceps muscle phenotype in chronic obstructive pulmonary disease (Copd); implications for stratified medicine? Muscle \& nerve. 2013 Oct;48(4):488-97. PubMed PMID: 23553751.

50. Merlini L, Nishino I, Consortium for Autophagy in Muscular D. 201st ENMC International Workshop: Autophagy in muscular dystrophies--translational approach, 1-3 November 2013, Bussum, The Netherlands. Neuromuscular disorders : NMD. 2014 Jun;24(6):546-61. PubMed PMID: 24746377. 


\section{Supplementary material}
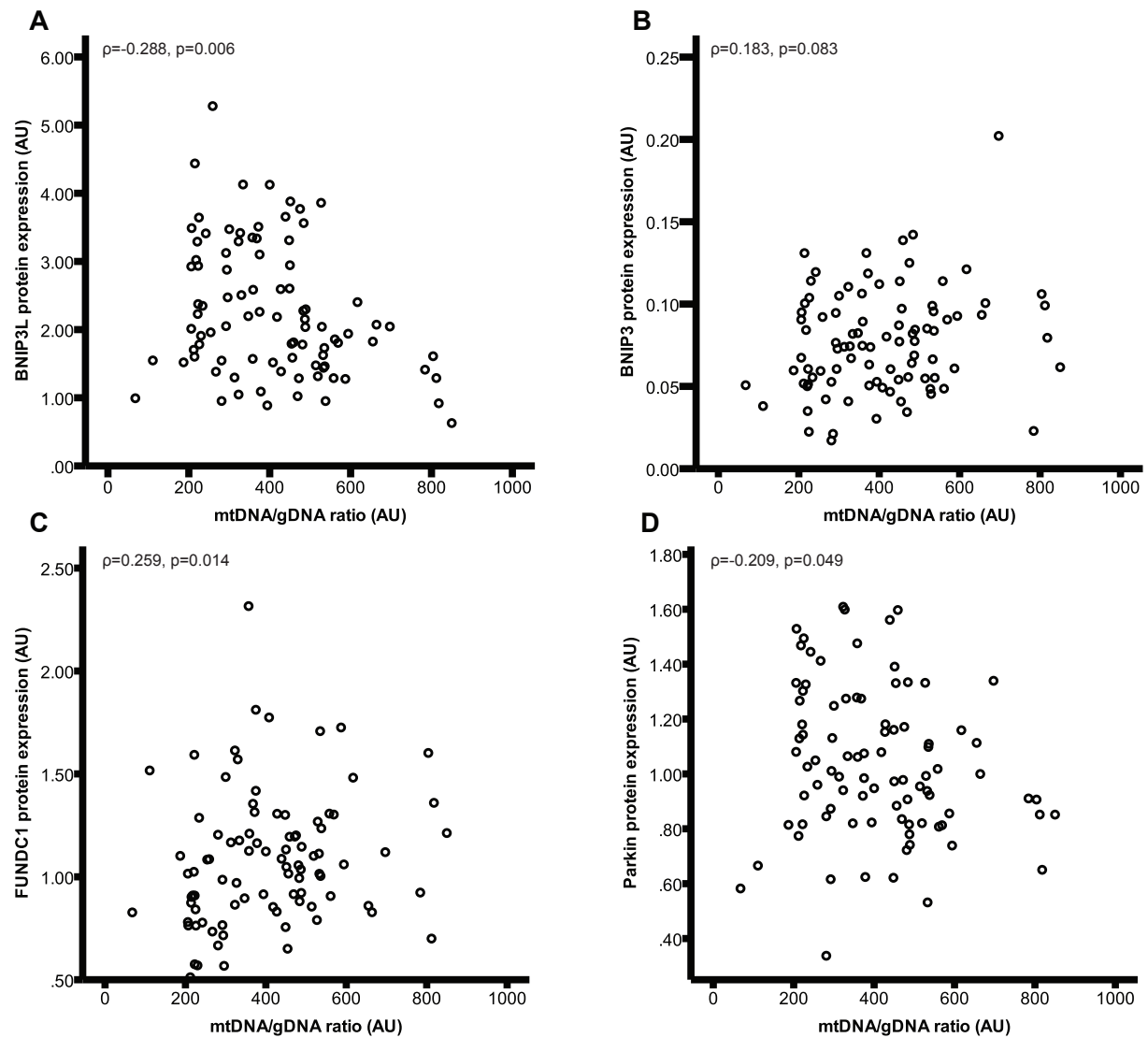

Supplementary Figure 1: Correlations of mitophagy markers with mtDNA/gDNA ratio. Correlations of mtDNA/gDNA ratio with BNIP3L (A), BNIP3 (B), FUNDC1 (C), and Parkin (D), are depicted in the group of COPD patients $(\mathrm{N}=89-91)$. 
A

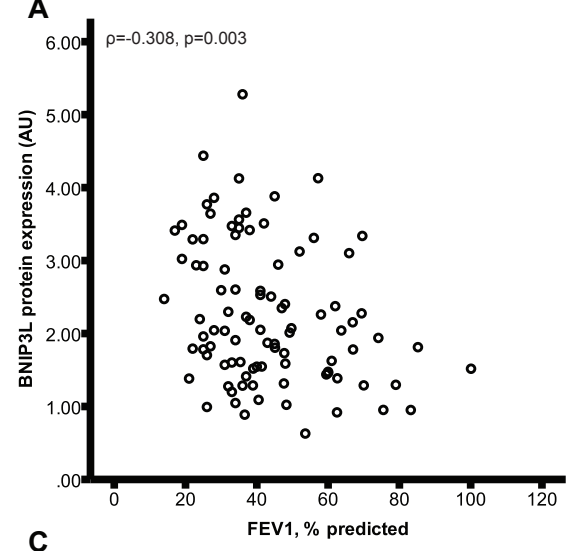

C

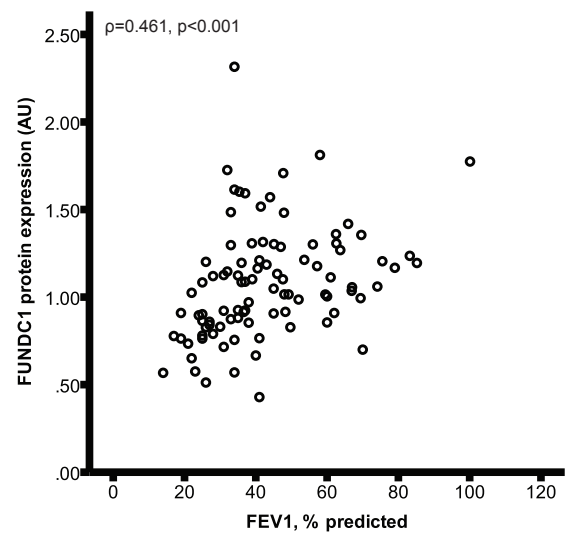

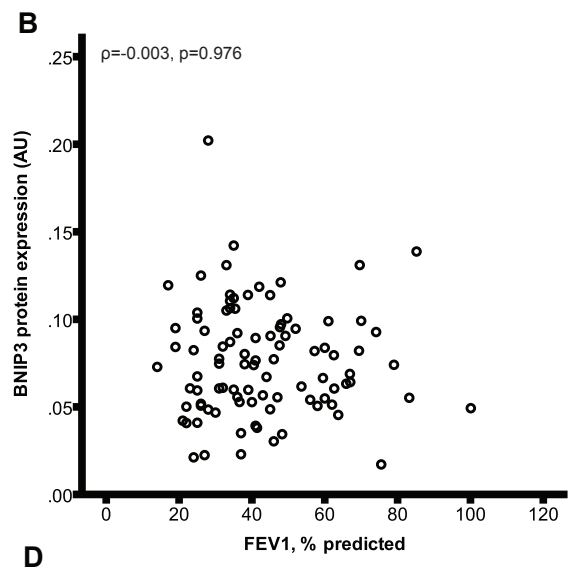

D

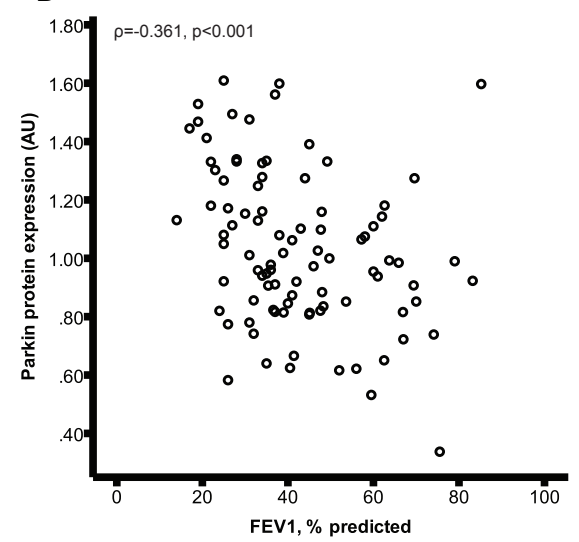

Supplementary Figure 2: Correlations of mitophagy markers with $\mathrm{FEV}_{1} \%$ predicted. Correlations of $\mathrm{FEV}_{1} \%$ predicted with BNIP3L (A), BNIP3 (B), FUNDC1 (C), and Parkin (D), are depicted in the group of COPD patients $(\mathrm{N}=92-94)$. 


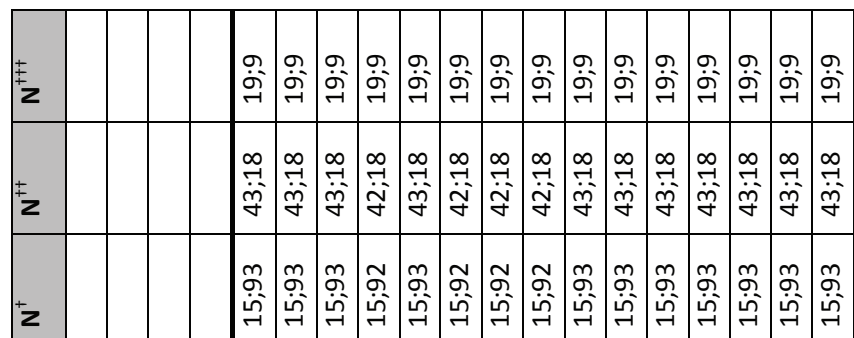

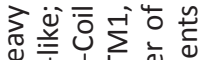

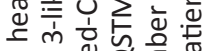

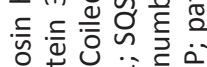

政然桴

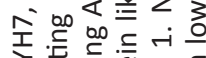

立

४⿻

品衰

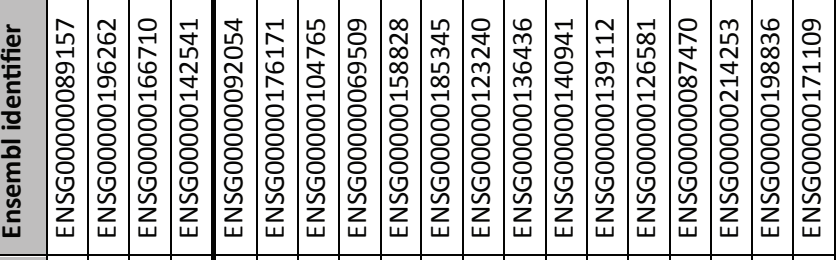

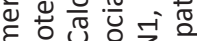

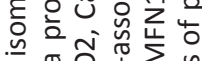

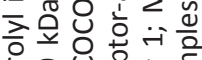

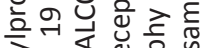
흠엉 흥흥 敦它是 을

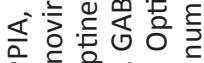

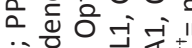

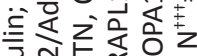
言 증 응

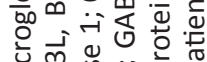

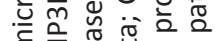

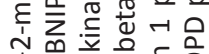

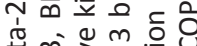
so 牙

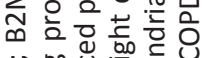
o.

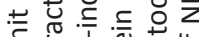

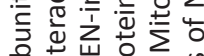

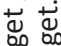

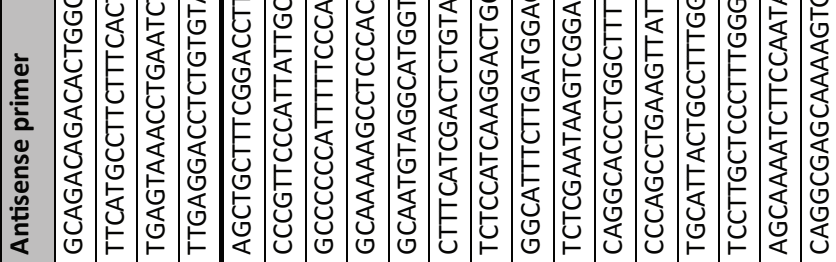

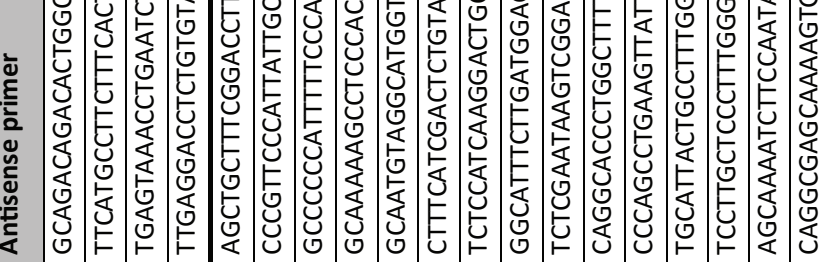

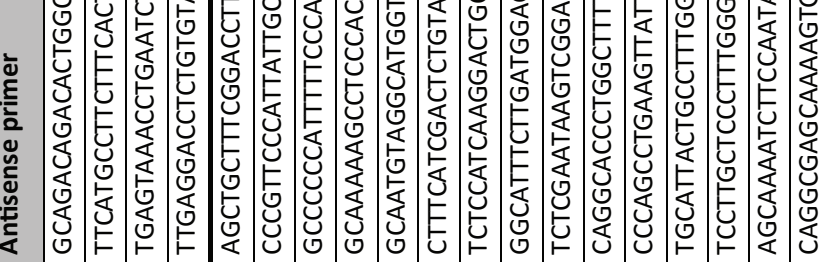

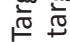

案

秀

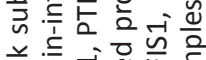

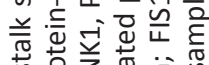

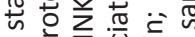
तٓ

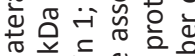

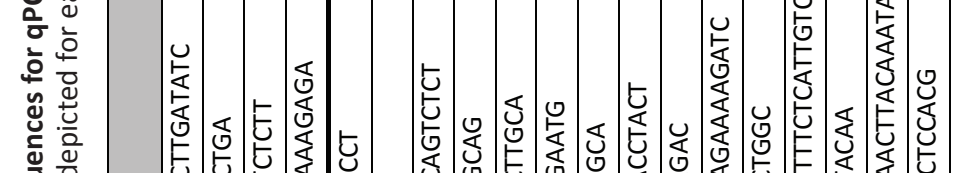

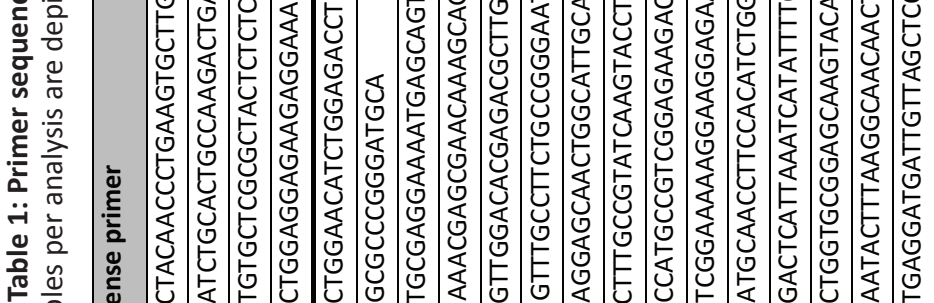

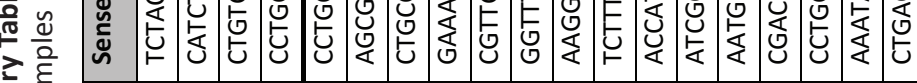

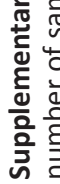

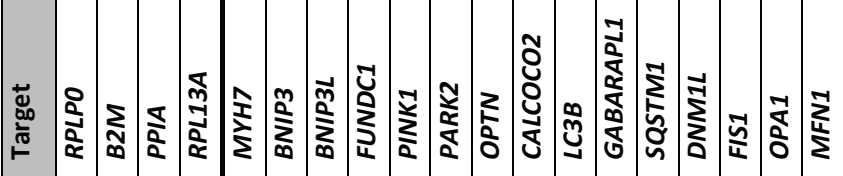

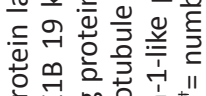

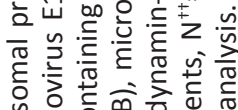

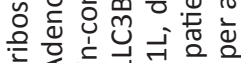

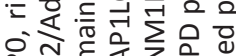

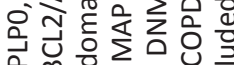

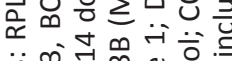

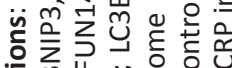

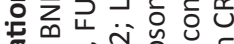

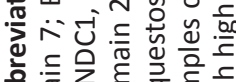

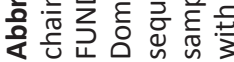


Supplementary Table 2: Primary antibodies for western blotting. Target protein, supplier of antibody, product number, research identifier, and specific number of samples per analysis are depicted for each target.

\begin{tabular}{|c|c|c|c|c|c|c|c|}
\hline Target & Supplier & Product nr. & Dilution & Research identifier & $\mathbf{N}^{+}$ & $\mathbf{N}^{\dagger+}$ & $\mathbf{N}^{t+\dagger}$ \\
\hline OXPHOS & MitoSciences & MS604 & $1: 1,000$ & RRID:AB_2629281 & $14 ; 95$ & $44 ; 19$ & $19 ; 9$ \\
\hline BNIP3 & Cell signaling & 3769 & $1: 1,000$ & RRID:AB_2259284 & $14 ; 94$ & $44 ; 18$ & $19 ; 9$ \\
\hline BNIP3L & Cell signaling & 12396 & $1: 1,000$ & RRID:AB_2688036 & $14 ; 94$ & $44 ; 18$ & $19 ; 9$ \\
\hline FUNDC1 & Santa Cruz & sc-133597 & $1: 500$ & RRID:AB_10609242 & $14 ; 94$ & $44 ; 18$ & $19 ; 9$ \\
\hline Parkin & Cell signaling & 4211 & $1: 1,000$ & RRID:AB_2159920 & $14 ; 92$ & $43 ; 18$ & $18 ; 9$ \\
\hline LC3B & Cell signaling & 2775 & $1: 1,000$ & RRID:AB_915950 & $14 ; 93$ & $43 ; 18$ & $19 ; 9$ \\
\hline GABARAPL1 & Proteintech & 11010-1-AP & $1: 500$ & RRID:AB_2294415 & $14 ; 94$ & $44 ; 18$ & $19 ; 9$ \\
\hline SQSTM1 & Cell signaling & 5114 & $1: 1,000$ & RRID:AB_10624872 & $14 ; 94$ & $44 ; 18$ & $19 ; 9$ \\
\hline DNM1L & Cell signaling & 8570 & $1: 1,000$ & RRID:AB_10950498 & $14 ; 94$ & $44 ; 18$ & $19 ; 9$ \\
\hline
\end{tabular}

Abbreviations: OXPHOS, oxidative phosphorylation; BNIP3, BCL2/Adenovirus E1B 19 kDa protein-interacting protein 3, BNIP3L, BCL2/Adenovirus E1B 19 kDa protein-interacting protein 3-like; FUNDC1, FUN14 domain-containing protein 1; LC3B (MAP1LC3B), microtubule associated protein 1 light chain 3 beta; GABARAPL1, GABA(A) receptor-associated protein like 1; SQSTM1, sequestosome 1; DNM1L, dynamin-1-like protein. $\mathrm{N}^{+}=$number of samples control; COPD patients, $\mathrm{N}^{+\dagger}=$ number of samples of NID-COPD; ID-COPD patients, $\mathrm{N}^{+++}=$number of samples of patients with low CRP; patients with high CRP included per analysis. 


\section{4}

Inflammation-induced alterations in key regulators of mitophagy and mitochondrial biogenesis in murine skeletal muscle

P.A. Leermakers, A.H.V. Remels, R.C.J. Langen, A.M.W.J. Schols, H.R. Gosker

Submitted 


\section{Abstract}

Both mitophagy, a selective mechanism for clearance of mitochondria, and mitochondrial biogenesis are key processes determining mitochondrial content and oxidative capacity of the musculature. Abnormalities in these processes could therefore contribute to deterioration of peripheral muscle oxidative capacity. Although it has been suggested that both mitophagy and mitochondrial biogenesis can be modulated by inflammatory mediators, it is unknown whether pulmonary inflammation affects these processes in skeletal muscle in vivo. To address this, we investigated multiple important constituents and molecular regulators of mitophagy and mitochondrial biogenesis in skeletal muscle over time in a murine model of pulmonary- and systemic inflammation induced by a single bolus of intra-tracheally (IT)-instilled lipopolysaccharide (LPS). IT-LPS instillation resulted in molecular patterns indicative of activation of mitophagy in skeletal muscle. This coincided with modulation of mRNA transcript abundance of genes involved in mitochondrial fusion and fission as well as an initial decrease and subsequent recovery of transcript levels of key proteins involved in the molecular regulation of mitochondrial biogenesis. However, no solid differences in markers for mitochondrial content were found. In conclusion, these data suggest that one bolus of IT-LPS results in the temporal modulation of mitochondrial clearance and biogenesis signalling, but is insufficient to result in reductions of mitochondrial content. 


\section{Introduction}

The mitochondrial network in skeletal muscle is highly dynamic and displays a remarkable degree of plasticity. While increased muscle mitochondrial oxidative capacity is generally associated with increased performance and endurance, impairments in muscle oxidative capacity are associated with a variety of metabolic aberrations, exercise intolerance and progressive disability. Processes involved in remodelling of the mitochondrial network include fusion and fission events, biogenesis of neworganellesand mitochondrialclearancebymitophagy(1).

Several chronic diseases, including Chronic Obstructive Pulmonary Disease (COPD) and Chronic heart failure (CHF) are characterised by impairments in skeletal muscle mitochondrial function (2-4). Moreover, these disorders are also associated with a chronic low-grade inflammatory state as e.g. identified by chronically increased levels of inflammatory mediators in the circulation and activation of inflammatory signalling in peripheral muscle $(3,5,6)$. Interestingly, muscle inflammatory signalling has been proposed as an important regulator of skeletal muscle mitochondrial remodelling. Indeed, abnormalities in mitochondrial function were found in skeletal muscle of murine inflammatory models $(7,8)$ and activation of muscle inflammatory signalling was reported to be associated with decreased metabolic gene expression in skeletal muscle (9). Moreover, activation of nuclear factor kappa B (NF-kB) inflammatory signalling potently reduced mitochondrial content and mitochondrial respiration in cultured skeletal muscle cells (6). Although it has been suggested that both mitophagy and mitochondrial biogenesis can be modulated by inflammatory mediators, it is unknown whether inflammation affects these processes in skeletal muscle in vivo.

Mitophagy, a process defined by selective autophagy of mitochondria (10), has received much attention in recent years as an essential mechanism of mitochondrial quality control and remodelling, and can roughly be divided in receptor-mediated mitophagy and ubiquitin-mediated mitophagy (11). During receptor-mediated mitophagy, a mitochondrial-based receptor-protein, e.g. BCL2/adenovirus E1B $19 \mathrm{kDa}$ protein interacting protein 3 (BNIP3), BNIP3 like (BNIP3L), or FUN14 domain containing 1 (FUNDC1), is activated. Subsequently, the activated receptor protein recruits and binds a mammalian homologue of yeast autophagy related protein (ATG) 8 , of which microtubule-associated protein 1 light chain 3 beta (MAP1LC3B or LC3B) and gamma-aminobutyric acid (GABA), a receptor-associated protein-like 1 (GABARAPL1) are the best described in mitophagy research (12-14). This recruitment subsequently facilitates 
autophagosomal membrane formation around the mitochondrion and predestines the organelle for degradation.

The ubiquitin-mediated mitophagy pathway is regulated by mitochondrial membrane potential. PTEN induced putative kinase 1 (PINK1), an important constituent of this pathway, is constantly broken down in healthy mitochondria. However, in case of mitochondrial dysfunction, PINK1 is stabilized and the E3 ubiquitin-protein ligase Parkin (PARK2) is recruited and activated. Subsequently, PARK2 is able to ubiquitinate several different mitochondrial substrates, including mitofusin (MFN)-1 and -2 (15) which recruit the LIR-containing autophagy adaptor protein Sequestosome-1 (SQSTM1). SQSTM1, like BNIP3(L) and FUNDC1 (16), is then able to recruit LC3B and facilitate autophagosomal membrane formation. Additionally, PINK1 is also able to recruit autophagy receptors (e.g. Optineurin (OPTN)) independently from PARK2, which are also able to recruit autophagy-related factors and promote mitochondrial autophagosome formation (17). As mentioned above, in addition to breakdown of mitochondria via mitophagy, muscle mitochondrial content is also highly determined by the biogenesis of new organelles, which is essentially controlled by the peroxisome proliferative activated receptor (PPAR), gamma, coactivator 1 (PPARGC1) signalling network (18). Key elements of this network include PPARGC1 alpha (PPARGC1A) and beta (PPARGC1B), as well as a multitude of transcription factors including PPAR alpha (PPARA) and delta (PPARD), estrogen related receptor, alpha (ESRRA), nuclear respiratory factor 1 (NRF1), GA repeat binding protein, alpha (GABPA) and transcription factor A, mitochondrial (TFAM), that collectively control the coordinated expression of key constituents of mitochondrial metabolic pathways. Illustrative of its significance in controlling muscle mitochondrial capacity, genetic activation of this pathway potently induces mitochondrial biogenesis (19) while its abrogation induces loss of mitochondrial content. Moreover, activation of the PPARGC1 pathway is a prerequisite for exercise-induced increases in muscle mitochondrial capacity (20) and abundance of these molecules has been shown to be reduced in peripheral muscle in COPD patients (21). Interestingly, PPARGC1 molecules have also been implicated in the molecular regulation of mitochondrial fusion and fission and mitophagy (22).

We have shown previously that inflammation and subsequent activation of inflammatory signalling detrimentally impacts mitochondrial biogenesis and the molecular regulation thereof by PPARGC1 in cultured muscle cells (9). However, whether an inflammatory process has the ability to influence the molecular regulation of mitophagy and mitochondrial biogenesis in peripheral skeletal 
muscle in vivo remains unexplored. Therefore, in the present study, we used an established in vivo model of (systemic) inflammation induced by intra-tracheally (IT)-instilled lipopolysaccharide (LPS). Importantly, we have shown previously that this model results, not only in prominent inflammation in the pulmonary compartment but also in increased circulatory levels of inflammatory mediators as well as activation of inflammatory signalling in the peripheral musculature (23-25). Our primary objective was to investigate whether or not molecular signalling patterns indicative of increased cellular mitophagy and decreased mitochondrial biogenesis are present in the skeletal musculature of mice acutely exposed to a single bolus IT-LPS. As a secondary objective, we explored whether potential alterations in these pathways may be associated with indications for decreased muscle mitochondrial content in this acute setting of IT-LPS administration.

\section{Materials and methods}

\section{Ethical approval}

Animal procedures were performed in accordance with the European Directive 2010/63/EU guidelines and conform the journal regulations (26). This study was performed under a protocol approved by the Institutional Animal Care Committee of Maastricht University (DEC-2007-040) in accordance to the National Institutes of Health guide for the care and use of Laboratory animals.

\section{Experimental animals}

The presented murine data concerns data from the genetic control group, expressing 6xhis/GFP-tagged ubiquitin on a FVB/N background (27), of a larger research project concerning multiple unrelated hypotheses (28), in order to reduce number of laboratory animals used. 12 week old male mice were housed in the animal care facility at Maastricht University in a temperature-controlled environment with $12 \mathrm{~h}-12 \mathrm{~h}$ light-dark cycle and fed ad libitum. Mice were anesthetized using i.p. xylazine $(3 \mathrm{mg} / \mathrm{kg})$ and ketamine $(75 \mathrm{mg} / \mathrm{kg})$, and subjected to one intra-tracheal instillation of $20 \mu \mathrm{g}$ lipopolysaccharide (IT-LPS) or saline (IT-NaCl), after which they were directly allowed to awaken. Mice were sacrificed after $7,24,48,72,96$, or $120 \mathrm{~h}$ by i.p. sodium pentobarbital $(115 \mathrm{mg} / \mathrm{kg}$ ) followed by exsanguination. Group sizes were as follows, IT-NaCl 7h $n=3$, IT-LPS $7 \mathrm{~h} n=5$, IT-NaCl 24-120h n=4, and IT-LPS 24-120h n=6. Gastrocnemius muscle was excised, snap-frozen in liquid nitrogen, and crushed into powder. A small proportion of data included in this manuscript (i.e. proportions of LC3B and SQSTM1 protein and mRNA expression data) has been previously published in a research project concerning unrelated hypotheses (28). 


\section{RNA extraction and qPCR}

RNA was extracted from 15 mg muscle powder using TRI Reagent ${ }^{\circledR}$-based separation methods (Sigma-Aldrich, Zwijndrecht, the Netherlands). $200 \mathrm{ng}$ of RNA was used for cDNA synthesis using the tetro cDNA synthesis kit (Bioline, Alphen aan de Rijn, the Netherlands) according to manufacturer's protocol. $4.4 \mu \mathrm{l}$ of $1 / 50$ diluted cDNA was used for quantitative PCR amplification using target and species specific primers (Table 1 ) and $2 X$ Sensimix $^{\mathrm{TM}} \mathrm{SYBR}^{\circledR}$ \& Fluorescein mix (Bioline, Alphen aan de Rijn, the Netherlands) on a LightCycler480 384-wells PCR machine (Roche, Almere, the Netherlands). Specificity of PCR amplification was tested with melt curve analysis and expression levels of genes of interest were corrected using a normalization factor calculated based on the expression of 3 different housekeepers (Rp/pO, Rp/13A and B2m) using the GeNorm software (Primerdesign, Southampton, USA).

\section{Protein extraction and Western Blotting}

$15 \mathrm{mg}$ muscle powder was homogenized in 350-400 $\mu \mathrm{l}$ IP lysis buffer (50 mM Tris, $150 \mathrm{mM} \mathrm{NaCl}, 10 \%$ glycerol, 0.5\% Nonidet P40, $1 \mathrm{mM}$ EDTA, $1 \mathrm{mM}$ Na3VO4, 5 mM NaF, 10 mM $\beta$-glycerophosphate, 1 mM Na4O7P2, 1 mM DTT, $10 \mu \mathrm{g} / \mu$ leupeptin, $1 \%$ apropeptin, $1 \mathrm{mM}$ PMSF, $\mathrm{pH}$ 7.4) with the Polytron PT 1600 E (Kinematica, Luzern, Switzerland). Lysates were incubated while rotating for $30 \mathrm{~min}$, and subsequently centrifuged at $20,000 \times \mathrm{g}$ for $30 \mathrm{~min}$ at $4{ }^{\circ} \mathrm{C}$. Protein concentrations were determined using the Pierce ${ }^{\mathrm{TM}}$ BCA Protein Assay Kit (Thermo Scientific, Landsmeer, \#23225) according to the manufacturer's protocol. Lysate $(1 \mu \mathrm{g} / \mu \mathrm{l})$ was aliquoted in sample buffer $(0.25 \mathrm{M}$ Tris- $\mathrm{HCl}, 8 \%$ (w/v) SDS, 40\% (v/v) glycerol, 0.4M DTT, 0.04\% (w/v) Bromphenol Blue, pH 6.8) and boiled for $5 \mathrm{~min}$ at $95^{\circ} \mathrm{C}$.

$10 \mu \mathrm{g}$ of protein per sample was run through a Criterion 26 -wells $12 \%$ precast gel (Bio-Rad Laboratories B.V., Veenendaal, the Netherlands) in 1x MES buffer (Bio-Rad Laboratories B.V., Veenendaal, the Netherlands) at 100 volts, and was subsequently blotted on a Nitrocellulose membrane (Bio-Rad Laboratories B.V., Veenendaal, the Netherlands) by electroblotting. At least two protein ladders were loaded on each gel (Precision Plus Protein ${ }^{\mathrm{TM}}$ All Blue Standards, Bio-Rad Laboratories B.V., Veenendaal, the Netherlands, Bio-Rad Laboratories B.V., \#161-0373).

Membranes were incubated in Ponceau S ((Sigma-Aldrich, Zwijndrecht, the Netherlands) for $5 \mathrm{~min}$ and washed with milliQ before they were imaged using the LAS-3000 (Fujifilm Life Sciences B.V., Tilburg, the Netherlands) or the Amersham ${ }^{\mathrm{TM}}$ Imager 600 (GE Healthcare Life Sciences, Eindhoven, the Netherlands). Total protein Ponceau S quantification was used as correction for 
Table 1: Primers used for qPCR

\begin{tabular}{|c|c|c|c|}
\hline & Sense primer & Antisense primer & Ensembl identifier \\
\hline RplpO & GGACCCGAGAAGACCTCCTT & GCACATCACTCAGAATTTCAATGG & ENSMUSG00000067274 \\
\hline Rp/13A & САCTCTGGAGGAGAAACGGAAGG & GCAGGCATGAGGCAAACAGTC & ENSMUSG00000074129 \\
\hline$B 2 m$ & CTTTCTGGTGCTTGTCTCACTGA & GTATGTTCGGCTTCCCATTCTC & ENSMUSG00000060802 \\
\hline Cxcl1 & TCGTCTTTCATATTGTATGGTCAACACG & TGCCCTACCAACTAGACACAAAATGTC & ENSMUSG00000029380 \\
\hline $\operatorname{Tnf}$ & GGGCCACCACGCTCTTC & TACAGGCTTGTCACTCGAATTTTG & ENSMUSG00000024401 \\
\hline$C x c / 2$ & CCCTGGTTCAGAAAATCATCCAAA & TTTGGTTCTTCCGTTGAGGGAC & ENSMUSG00000058427 \\
\hline Nfkbia & GCTACCCGAGAGCGAGGAT & GCCTCCAAACACACAGTCATCA & ENSMUSG00000021025 \\
\hline Bnip3 & AGGTTTTCCTTCCATCTCTGTTACTG & TGTGTGAACAGAAGTCAGATCCAAA & ENSMUSG00000078566 \\
\hline Bnip3l & AGTCGGGACAGAGCAGCTCAAG & TCAAACATGATCTGCCCATCTTCTT & ENSMUSG00000022051 \\
\hline Fundc1 & CGAGTATTTGGCCACAGTTCC & CCACTGTGACTGGCAACCTG & ENSMUSG00000025040 \\
\hline Pink1 & GTCCTGAAGGGAGCAGACG & TTAAGATGGCTTCGCTGGAG & ENSMUSG00000028756 \\
\hline Park2 & CTGGCTGTCCCAACTCCCT & CCTCGGCCCCATACTGC & ENSMUSG00000023826 \\
\hline Optn & GAGCTGCAGGTGGAGAGCAT & CCACCTTTTCTGCCTGTTGC & ENSMUSG00000026672 \\
\hline Ndufb3 & ACAGACAGTGGAAAATTGAAGGG & GCCCATGTATCTCCAAGCCT & ENSMUSG00000026032 \\
\hline Sdhb & AATTTGCCATTTACCGATGGGA & AGCATCCAACACCATAGGTCC & ENSMUSG000000009863 \\
\hline Cyc1 & GCATTCGGAGGGGTTTCCAG & CCGCATGAACATCTCCCCA & ENSMUSG00000022551 \\
\hline$m t-\operatorname{Cox} 2$ & CCATCCCAGGCCGACTAA & ATTTCAGAGCATTGGCCATAGAA & ENSMUSG00000064354 \\
\hline Ppargc1a & CAACAATGAGCCTGCGAACA & CTTCATCCACGGGGAGACTG & ENSMUSG00000029167 \\
\hline Ppargc1b & ACCCTGAGAAAGCGCAATGA & CCCAGATGAGGGAAGGGACT & ENSMUSG00000033871 \\
\hline Ppara & ACTACGGAGTTCACGCATGTG & TTGTCGTACACCAGCTTCAGC & ENSMUSG00000022383 \\
\hline Ppard & AGGCCCGGAGCATCCTCA & TGGATGACAAAGGGTGCGTTG & ENSMUSG00000002250 \\
\hline Tfam & CCGGCAGAGACGGTTAAAAA & TCATCCTTTGCCTCCTGGAA & ENSMUSG00000003923 \\
\hline Nrf1 & AGCCACATTGGCTGATGCTT & GGTCATTTCACCGCCCTGTA & ENSMUSG00000058440 \\
\hline Gabpa & TGCTGCACTGGAAGGCTACA & TTACCCAAACCACCCAATGC & ENSMUSG00000008976 \\
\hline Esrra & GGCGGACGGCAGAAGTACAAA & GCGACACCAGAGCGTTCAC & ENSMUSG00000024955 \\
\hline Dnm1I & TGCCCACTGAGCAATCTCAA & TGCTAACACATTTAGGCAGTGTGTACT & ENSMUSG00000022789 \\
\hline Fis1 & GGGCAACTACCGGCTCAAG & GCCATGCCTACCAGTCCATC & ENSMUSG00000019054 \\
\hline Mtfp1 & CCACCACACTTGGACTGCTG & GGCTTCTCCACTGACGGGTA & ENSMUSG00000004748 \\
\hline Opa1 & GGGAAAACCAGTGTGCTGGA & AACAAGGCCACATGGTGAGG & ENSMUSG000000038084 \\
\hline Mfn1 & GCTGGCTGTCTTGTGCATGT & TCCAGCTCTGTGGTGACATCTG & ENSMUSG00000027668 \\
\hline Mfn2 & CAGGGGTATCAGCGAAGTGC & ACCAATCCCAGATGGCAGAA & ENSMUSG00000029020 \\
\hline$L c 3 b$ & GAGCAGCACCCCACCAAGAT & CGTGGTCAGGCACCAGGAA & ENSMUSG00000031812 \\
\hline Gabarapl1 & СCCTCCCACCAGTGCTACCAT & TCATCACTGTAGGCCACATACAGAAAA & ENSMUSG00000030161 \\
\hline Sqstm1 & GCAGCTGCTCTTCGGAAGTC & CCCACCGACTCCAAGGCTAT & ENSMUSG00000015837 \\
\hline
\end{tabular}

gel-loading. Subsequently, membranes were washed, blocked with $3 \%$ non-fat, dried milk (Campina, Amersfoort, the Netherlands) in TBS-Tween-20 (0.05\%) for $1 \mathrm{~h}$, washed, and incubated overnight at $4{ }^{\circ} \mathrm{C}$ with different protein-specific primary antibodies against: TFAM (Millipore Cat\# DR1071, RRID:AB_10682431), NRF1 (Abcam Cat\# ab55744, RRID:AB_2154534), PPARGC1A (Cell Signalling Technology Cat\# 2178S, RRID:AB_823600), BNIP3 (Cell Signalling Technology Cat\# 3769S, RRID:AB_2259284), BNIP3L (Cell Signalling Technology Cat\# 12396, RRID:AB_2688036), LC3B(CellSignalling TechnologyCat\#2775, RRID:AB_915950), 
SQSTM1 (Cell Signalling Technology Cat\# 5114, RRID:AB_10624872), PARK2 (Cell Signalling Technology Cat\# 4211, RRID:AB_2159920), DNM1L (Cell Signalling Technology Cat\# 8570, RRID:AB_10950498), FUNDC1 (Santa Cruz Biotechnology Cat\# sc-133597, RRID:AB_10609242), GABARAPL1 (Proteintech Group Cat\# 11010-1-AP, RRID:AB_2294415), and oxidative phosphorylation (OXPHOS) complex subunits (MitoScience LLC Cat\# MS604, RRID:AB_2629281) all diluted in 3\% non-fat, dried milk or bovine serum albumin in TBS-Tween-20. Membranes were washed and incubated with HRP-labelled, primary antibody-specific, secondary antibody (\#BA-9200, \#BA-1000, Vector Laboratories, Amsterdam, the Netherlands) (1:10,000 diluted in 3\% non-fat, dried milk in TBS-Tween-20) for $1 \mathrm{~h}$ at room temperature. Membranes were washed and incubated with either 0.5x SuperSignal West Pico Chemiluminescent Substrate or $0.25 x$ SuperSignal West Femto Chemiluminescent Substrate (Thermo Scientific, Landsmeer, the Netherlands) for $5 \mathrm{~min}$, depending on the expected signal strength. Photographs were taken with the LAS-3000 or Amersham ${ }^{\mathrm{TM}}$ Imager 600 and analysed with ImageQuant TL software (GE Healthcare Life Sciences, Eindhoven, the Netherlands).

\section{Enzyme activity assays}

$15 \mathrm{mg}$ muscle powder was mixed and homogenized in $240 \mu \mathrm{l}$ SET buffer ( $250 \mathrm{mM}$ sucrose, 2 mM EDTA, 10 mM Tris, pH 7.4) using the Mini-BeadBeater (Biospec, Bartlesville, U.S.A.) for $30 \mathrm{sec}$. The solution was snap frozen in liquid nitrogen, defrosted and incubated on ice for $30 \mathrm{~min}$, and subsequently centrifuged at $20,000 \times \mathrm{g}$ for $10 \mathrm{~min}$ at $4^{\circ} \mathrm{C} .12 \mu \mathrm{l} 10 \% \mathrm{BSA}$ was added to $108 \mu \mathrm{l}$ supernatant (resulting in $1 \% \mathrm{BSA}$ end concentration), which was stored at $-80^{\circ} \mathrm{C}$. Protein concentration was determined by the Pierce ${ }^{\mathrm{TM}}$ BCA Protein Assay Kit in the remaining supernatant.

Enzymatic assays were performed as described previously for both the citrate synthase (CS) assay and $\beta$-hydroxyacyl-CoA dehydrogenase (HADH) assay. Absorbance at specific wavelengths was measured using the Multiscan Spectrum machine (Thermo Lab Systems, Landsmeer, the Netherlands) (29). Slope determination was performed, and corrected for total protein concentration.

\section{Mitochondrial DNA copy number}

Total DNA was extracted from $15 \mathrm{mg}$ muscle powder using the GenElute Mammalian Genomic DNA Miniprep kit (Sigma-Aldrich, Zwijndrecht, the Netherlands) according to manufacturer's protocol. $4.4 \mu \mathrm{l} 1 / 10$ diluted DNA was used for qPCR as described above, using mitochondrial DNA (mtDNA) or genomic DNA (gDNA) -specific primers (Table 2). mtDNA/gDNA ratio was determined by dividing relative quantity of mtDNA by the relative quantity of gDNA. 
Table 2: Primers used for assessment of mitochondrial DNA copy number

\begin{tabular}{|l|l|l|l|}
\hline & Sense primer & Antisense primer & Ensembl identifier \\
\hline $\boldsymbol{m} \boldsymbol{t}$ - $\mathbf{N d} \mathbf{1}$ & CAGGATGAGCCTCAAACTCC & GGTCAGGCTGGCAGAAGTAA & ENSMUSG00000064341 \\
\hline $\mathbf{B 2} \boldsymbol{m}$ & GGGAAGTCTTAGGGAGGAGCA & AGCTCTCAAGAACTGTGCCC & ENSMUSG00000060802 \\
\hline
\end{tabular}

\section{Statistics}

Data is depicted as box plots indicating median and interquartile range, with whiskers indicating minimum and maximum, as absolute numbers or as fold change compared to time-matched IT-NaCl. Data from IT-LPS mice $(n=5-6)$ was compared with the data form IT-NaCl mice $(n=3-4)$ within individual time-points only, using a Mann-Whitney $U$ test. Samples were not subjected to repeated hypotheses. All statistical analyses were performed using IBM SPSS 22 software.

\section{Results}

\section{Regulation of mitophagy in skeletal muscle in response to IT-LPS}

In line with previous work, IT-LPS instillation in our study resulted in significant reductions in body- as well as muscle weight in the first few days post instillation, indicative of the successful instillation of LPS (23) (Fig. 1 A-B). In addition, we observed increased transcript levels of several inflammatory genes in m.gastrocnemius in response to IT-LPS (Fig. 1 C-F).

To determine the impact of a single bolus of IT-LPS on key processes regulating mitochondrial content, we first assessed mRNA transcript levels and protein abundance of key proteins involved in mitophagy in gastrocnemius muscle at several time-points after IT-LPS instillation. In the first few days post instillation, muscle transcript levels of Bnip3 and Bnip3/ were significantly higher in mice subjected to IT-LPS compared with to IT-NaCl-instilled animals (Fig. 2 A-B). In addition, muscle Fundc1 and Optn transcript levels were lower $72 \mathrm{~h}$ post instillation, while Pink1 and Park2 mRNA levels were largely unaltered at all time-points in the LPS-subjected mice compared with the control group (Fig. 2 C-F).

Western blot analysis revealed two distinct bands for the BNIP3L protein. The band corresponding with the expected molecular weight was termed BNIP3L, while the extra band was termed BNIP3L-II. Specificity of both bands was verified by using a BNIP3L-specific siRNA construct (data not shown). While BNIP3L protein levels in skeletal muscle of mice subjected to IT-LPS were lower compared with controls $72 \mathrm{~h}$ post instillation, BNIP3L-II protein levels were found to be significantly higher $24 \mathrm{~h}-96 \mathrm{~h}$ post instillation in the IT-LPS mice, with the 
A

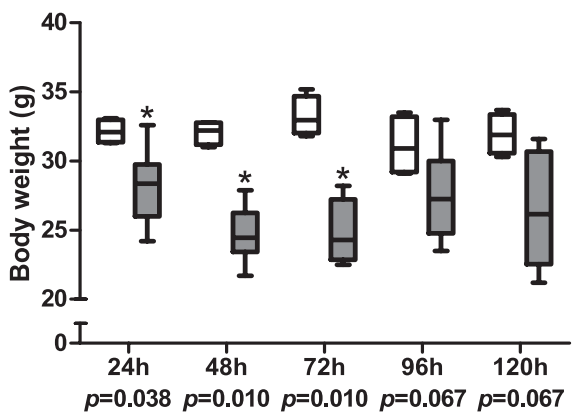

B $\square$ IT- $-\mathrm{NaCl}$ IT-LPS

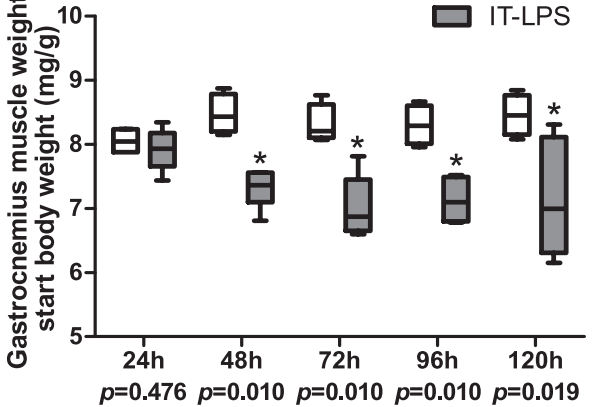

C

D
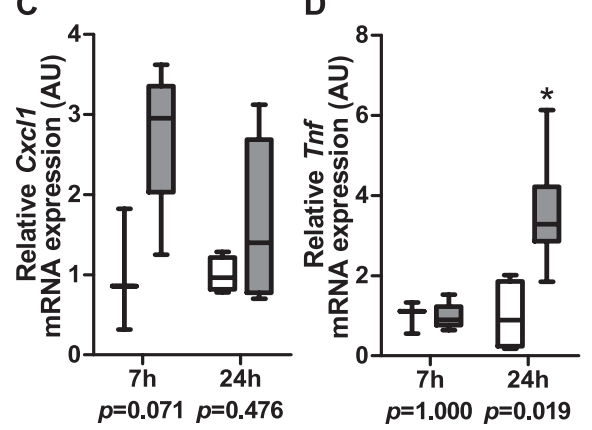

F

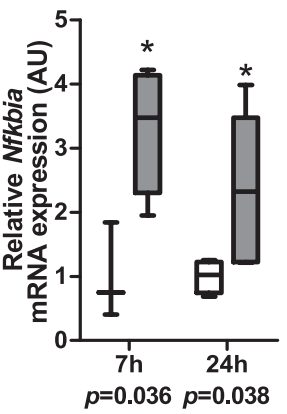

Figure 1: Body weight, $m$. gastrocnemius weight and $m$. gastrocnemius inflammatory mRNA expression. Body weight (A), $m$. gastrocnemius weight corrected for starting body weight (B). mRNA expression levels of Cxcl1 (C), Tnf (D), Cxc/2 (E) and Nfkbia (F) in m. gastrocnemius are depicted. Data is presented as box plots indicating median and interquartile range, with whiskers indicating $\min$ and max. n.d. $=$ not detectable. $p$-value and significant differences are depicted between groups within each time-point $* p<0.05$.

largest difference observed $48 \mathrm{~h}$ post instillation (Fig. 3 A-B). BNIP3 protein levels showed a similar pattern in response to IT-LPS (Fig. 3 C). In addition, FUNDC1 protein levels were significantly lower and PARK2 protein levels were significantly higher at respectively $72 \mathrm{~h}$ and $96 \mathrm{~h}$ post instillation in the IT-LPS mice (Fig. $3 \mathrm{D}-\mathrm{E}$ ).

Since mitophagy requires several general autophagy-related proteins for generating the autophagosomal membrane and priming the autophagosome to the mitochondria, we studied these proteins as well. In the first days post instillation, transcript levels of the genes coding for the BNIP3 binding partner LC3B (24h, 48h), the BNIP3L binding partner GABARAPL1 $(24 \mathrm{~h}, 48 \mathrm{~h})$, and the PINK1/PARK2-related autophagy receptor SQSTM1 (24h-72h) were significantly higher in mice subjected to IT-LPS compared with mice subjected to IT-NaCl (Fig. 4 A-C). Moreover, the ratio of LC3B-II/LC3B-I protein (24h, 48h) as well as protein levels of GABARAPL1 (48h), and SQSTM1 $(48 \mathrm{~h}, 72 \mathrm{~h})$ were higher during the first days post instillation in the IT-LPS mice compared with the control group (Fig. 4 D-H). 
A

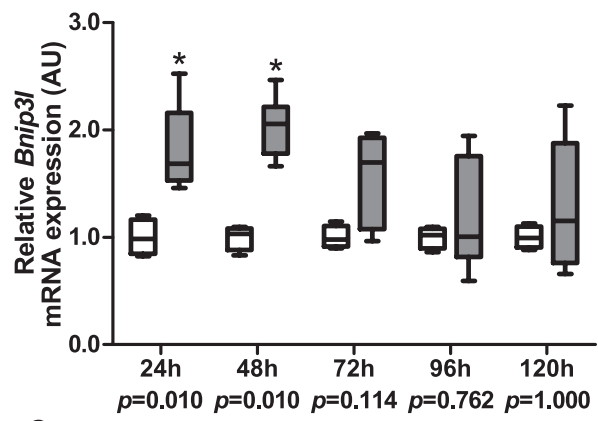

C

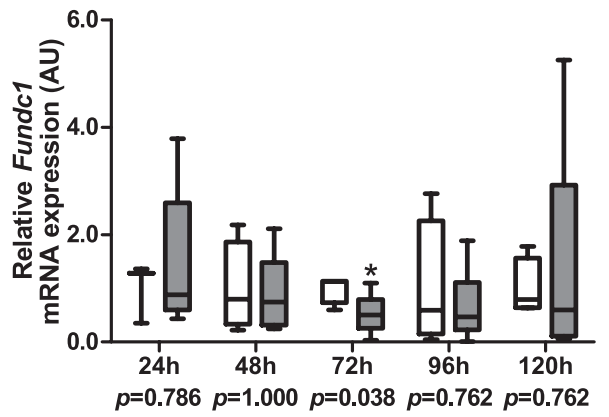

E

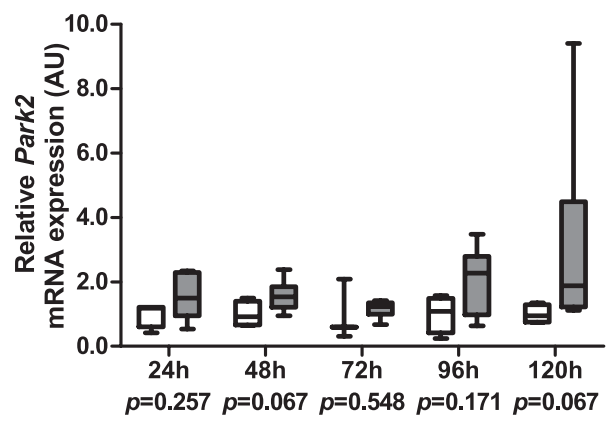

B

IT-NaCl

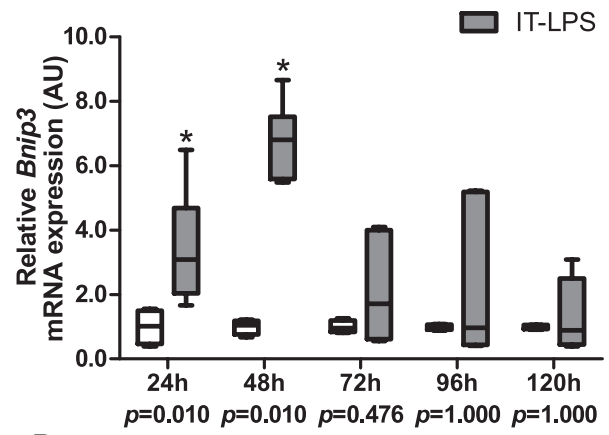

D

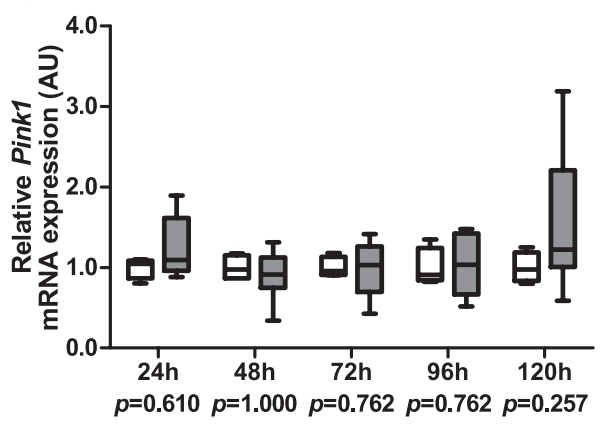

$\mathbf{F}$

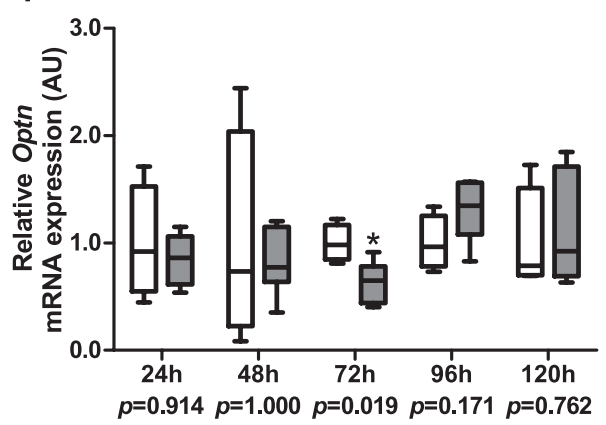

Figure 2: Mitophagy-associated mRNA expression in $m$. gastrocnemius in response to IT-LPS. mRNA expression levels of Bnip3l (A), Bnip3 (B), Fundc1 (C), Pink1 (D), Park2 (E) and Optn (F) in m. gastrocnemius are depicted. Data is presented as box plots indicating median and interquartile range, with whiskers indicating min and max. $p$-value and significance is depicted between groups within each time-point $* p<0.05$.

\section{Regulation of mitochondrial biogenesis in skeletal muscle in response to IT-LPS} In order to assess whether IT-LPs instillation impacts the PPARGC1 network in peripheral muscle, we subsequently measured transcript and protein levels of some of its key constituents. Ppargc1a transcript levels were largely unchanged while Ppargc1b transcript levels were dramatically lower in the IT-LPS group in the first few days post instillation (Fig. 5 A-B). Moreover, mice subjected to IT-LPS 
A

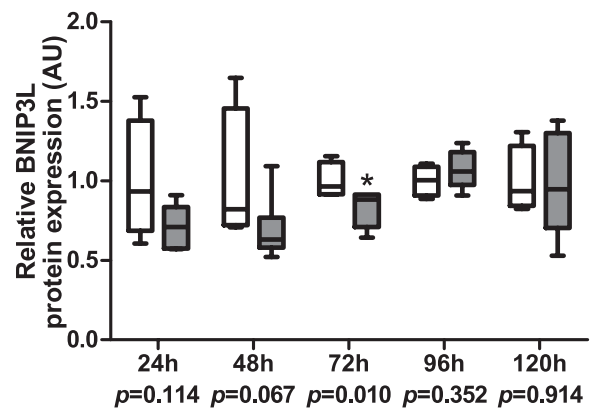

C

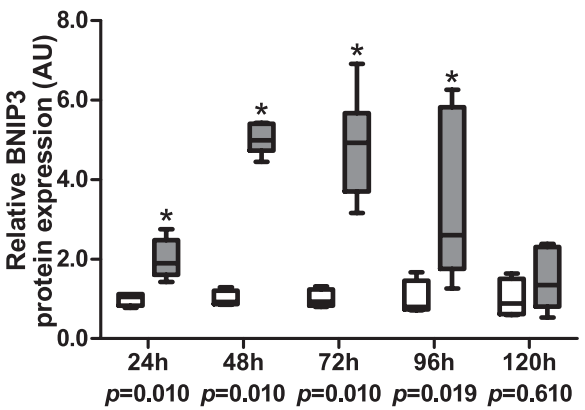

E

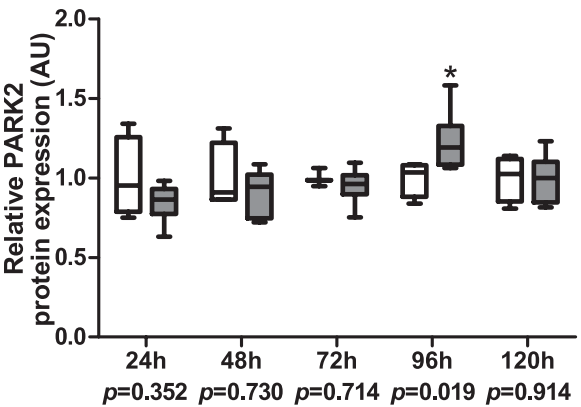

B

$\mathrm{IT}-\mathrm{NaCl}$

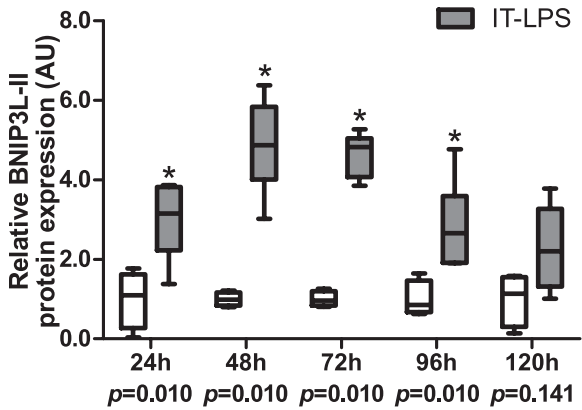

D

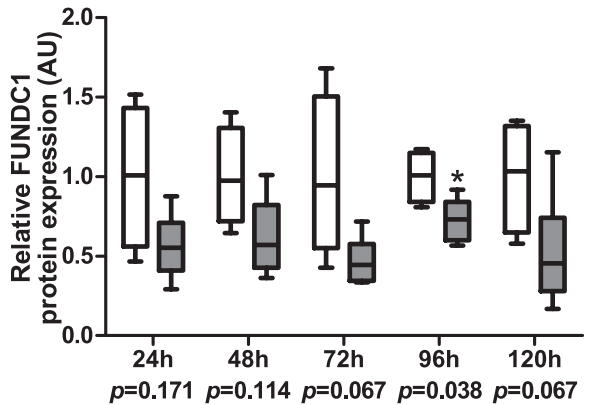

$\mathbf{F}$
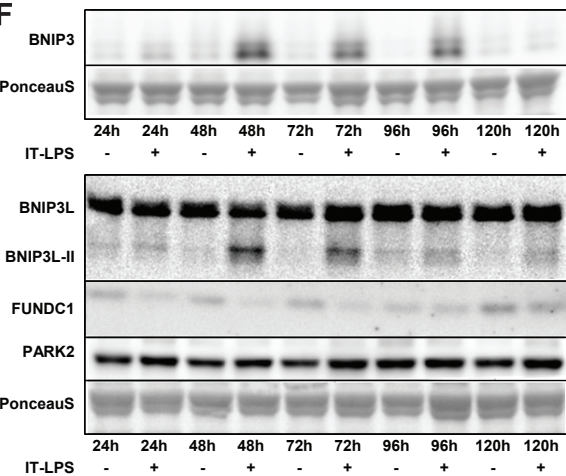

$\Delta$ Figure 3: Mitophagy-associated protein expression in $m$. gastrocnemius in response to IT-LPS. Protein levels of BNIP3L (A), BNIP3L-II (B), BNIP3 (C), FUNDC1 (D), and PARK2 (E) in m. gastrocnemius are depicted. Representative immunoblots and a representative part of Ponceau $S$ staining are shown, with adjusted contrast equally applied to whole photograph (F). Samples were equally divided over multiple gels which were derived and processed in parallel. Data is presented as box plots indicating median and interquartile range, with whiskers indicating $\min$ and $\max . p$-value and significance is depicted between groups within each time-point ${ }^{*} p<0.05$.

Figure 4: Autophagy-associated protein and mRNA expression in $m$. gastrocnemius in response to ITLPS. mRNA expression levels of $L c 3 b$ (A), Gabarapl1 (B) and sqstm1 (C) in $m$. gastrocnemius are depicted. Protein levels of LC3B (D-F), GABARAPL1 (G) and SQSTM1 (H) in $m$. gastrocnemius are depicted. Representative immunoblots and a representative part of Ponceau $S$ staining are shown, with cropped photographs indicated by black boxes, with adjusted contrast equally applied to whole photograph (I). For LC3B and SQSTM1, samples were grouped by time-point over different gels which were corrected for loading controls. For GABARAPL1, all samples were equally divided over multiple gels which were derived and processed in parallel. Data is presented as box plots indicating median and interquartile range, with whiskers indicating min and max. $p$-value and significance is depicted between groups within each time-point ${ }^{*} p<0.05$. 
A

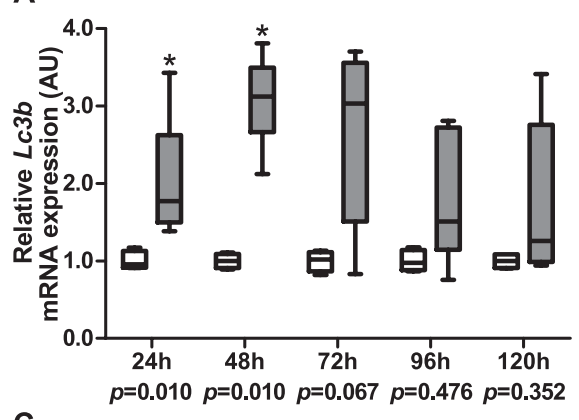

C

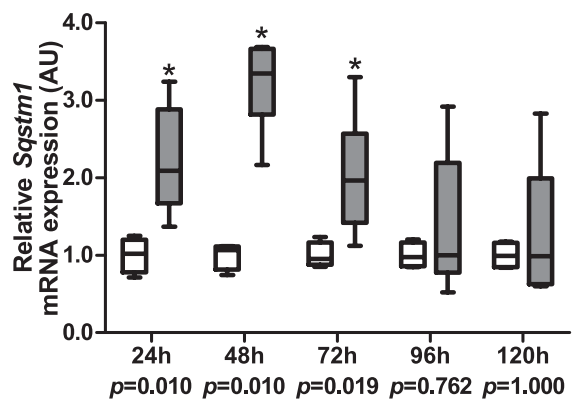

E

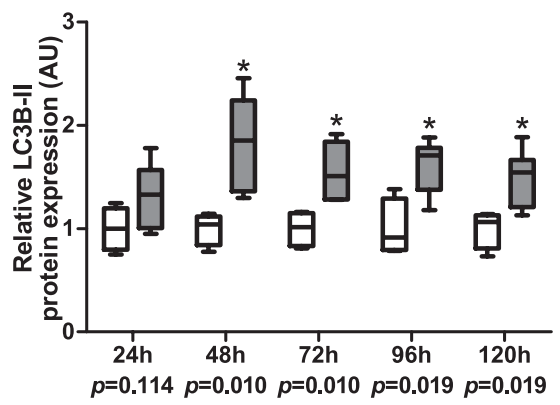

G

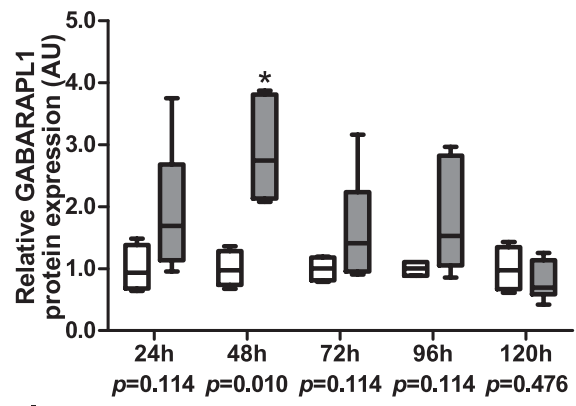

I

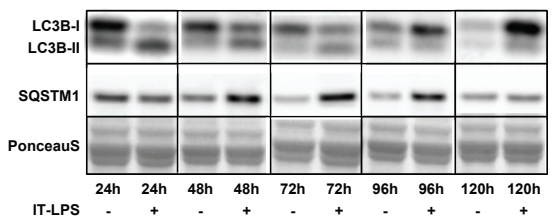

B $\square$ IT-NaCl

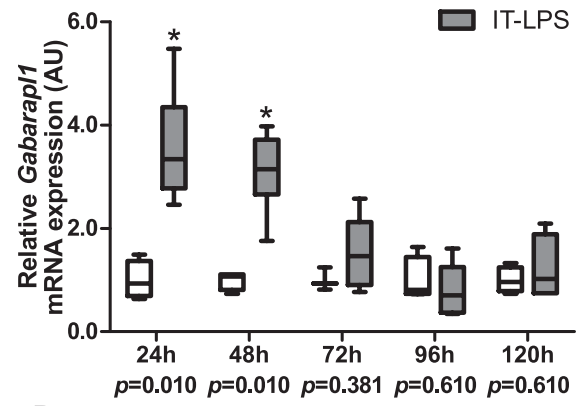

D

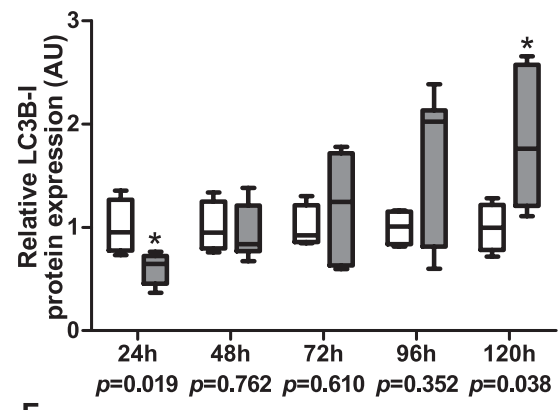

$\mathbf{F}$

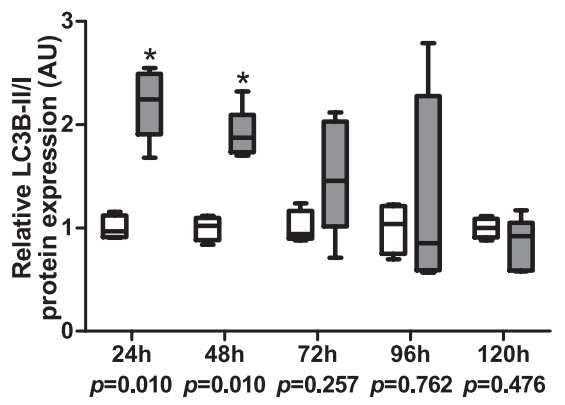

H
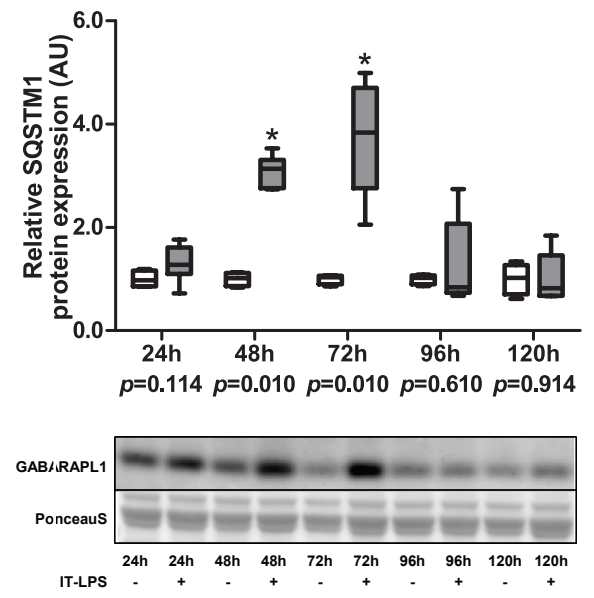
A

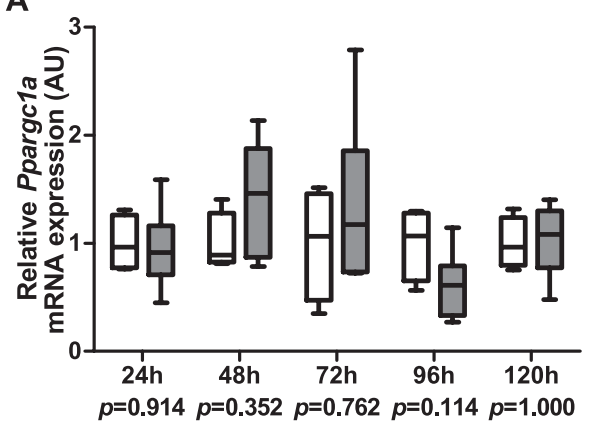

C

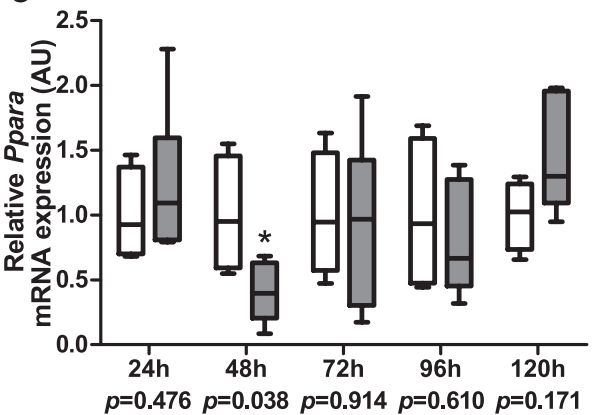

E

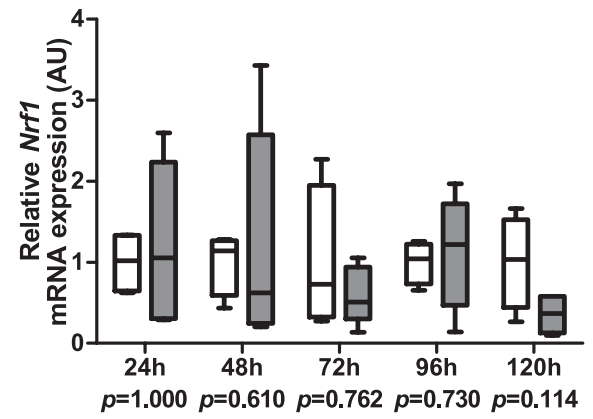

G

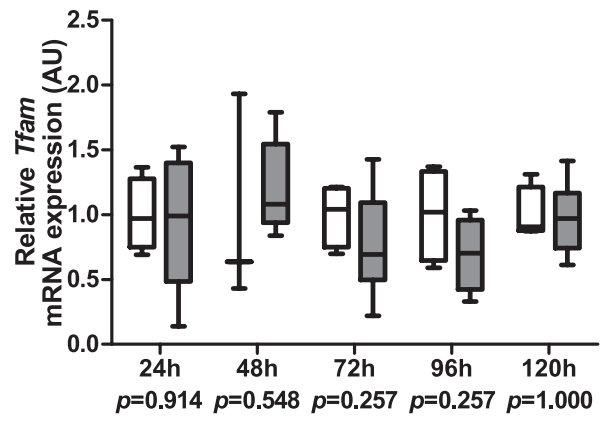

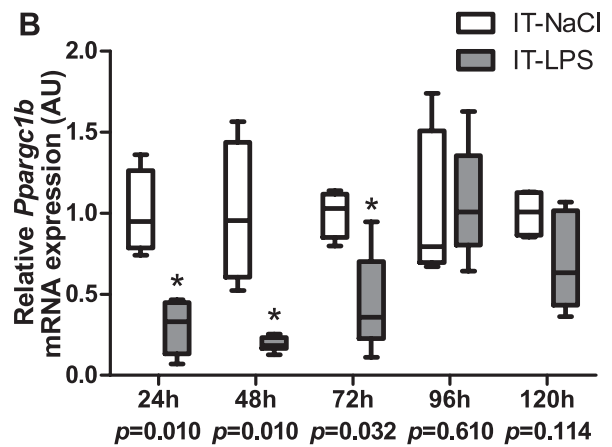

D

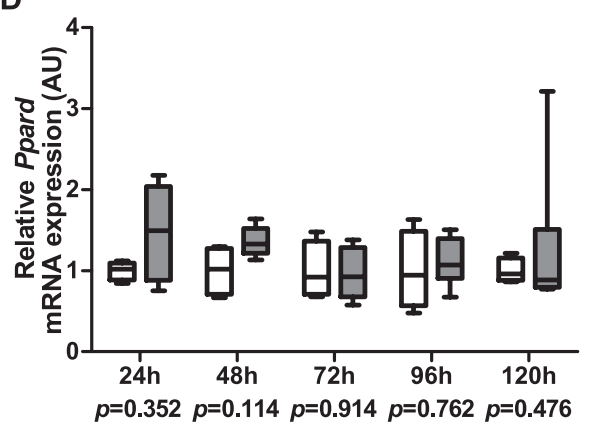

$\mathbf{F}$

H
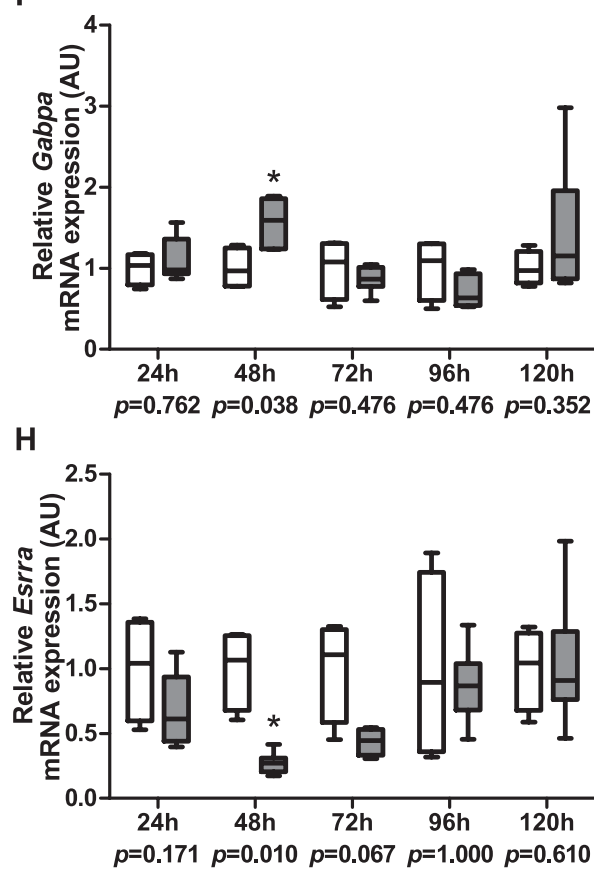

Figure 5: mRNA expression of key regulators of mitochondrial biogenesis in $\mathbf{m}$. gastrocnemius in response to IT-LPS. mRNA expression levels of Ppargc1a (A), Ppargc1b (B), Ppara (C), Ppard (D), $\operatorname{Nrf1}(\mathbf{E})$, Gabpa (F), Tfam (G), and Esrra (H) in m. gastrocnemius are depicted. Data is presented as box plots indicating median and interquartile range, with whiskers indicating min and max. $p$-value and significance is depicted between groups within each time-point $* p<0.05$. 
displayed lower transcript levels of Ppara and Esrra, and higher transcript levels of Gabpa $48 \mathrm{~h}$ post instillation. No differences in mRNA abundance of Tfam, Ppard or Nrf were observed at any time-point (Fig. 5 C-H). In addition, no differences were found in protein levels of PPARGC1A, NRF1 and TFAM in IT-LPS-treated animals compared with controls at any time-point (Fig. 6 A-C).

Subsequently, we studied if the abovementioned changes in expression levels of key regulators of mitochondrial biogenesis were associated with transcript levels of different OXPHOS subunits. Indeed, transcript levels of all tested nuclear-encoded OXPHOS subunits (complex I-III) as well as the mitochondrialencoded OXPHOS subunit Cox II (Complex IV) were, or tended to be, lower in mice subjected to IT-LPS compared with mice subjected to IT-NaCl, with differences in general being most pronounced $72 \mathrm{~h}$ post instillation (Fig. $7 \mathrm{~A}-\mathrm{D}$ ).

A

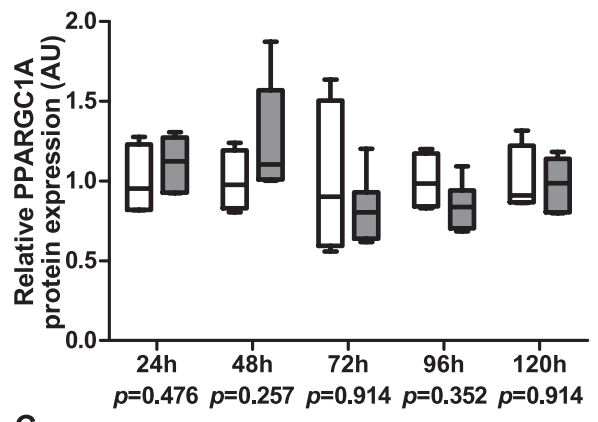

$\mathrm{C}$

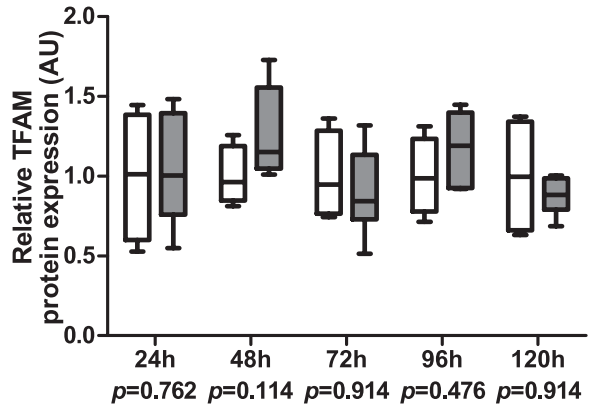

B

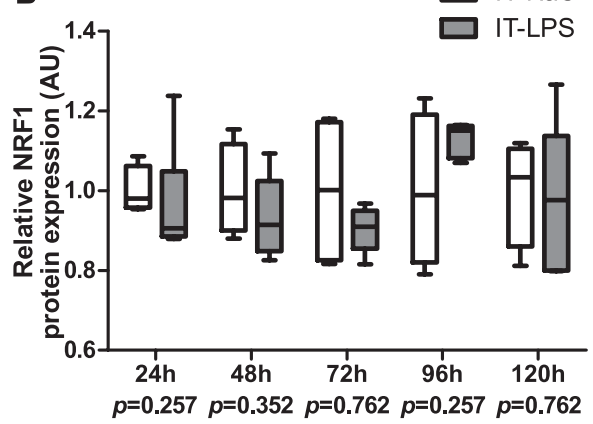

D

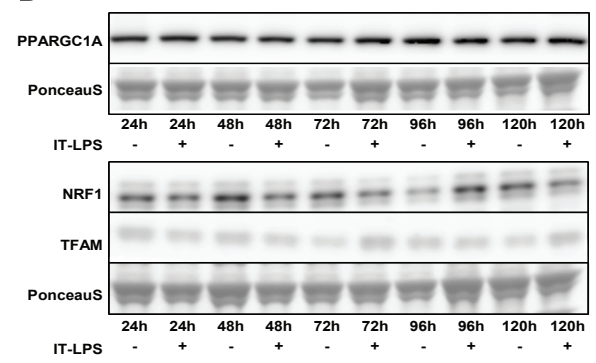

Figure 6: Protein expression of regulators of mitochondrial biogenesis in $\mathrm{m}$. gastrocnemius in response to IT-LPS. Protein expression of PPARGC1A (A), NRF1 (B) and TFAM (C) in m. gastrocnemius are depicted. Representative immunoblots and a representative part of Ponceau $S$ staining are shown, with adjusted contrast equally applied to whole photograph (D). Samples were equally divided over multiple gels which were derived and processed in parallel. Data is presented as box plots indicating median and interquartile range, with whiskers indicating min and max. $p$-value and significance is depicted between groups within each time-point ${ }^{*} p<0.05$. 
Expression levels of mitochondrial fission and fusion genes in skeletal muscle in response to IT-LPS. As mitochondrial fusion and fission are key events involved in mitochondrial remodelling by mitophagy and mitochondrial biogenesis, we next investigated mRNA transcript- and protein levels of proteins known to be involved in mitochondrial fusion and fission. As illustrated in Fig. 8, transcript abundance of the mitochondrial fission mediators dynamin-1-like (DNM1L) (48h-96h) and mitochondrial fission process 1 (MTFP1) (24h-96h) were significantly lower post instillation in mice subjected to IT-LPS compared with mice subjected to IT-NaCl while mitochondrial fission gene 1 (Fis1) transcript

A

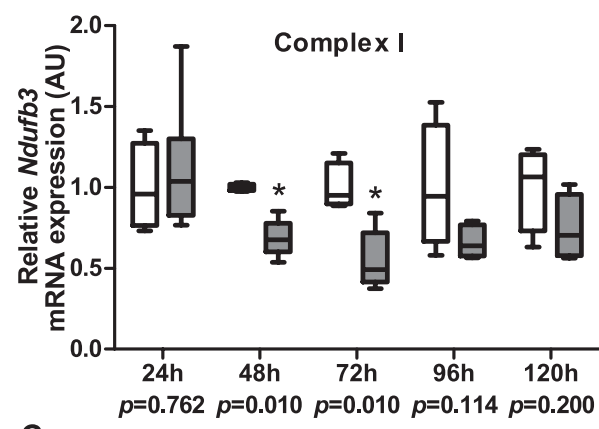

C

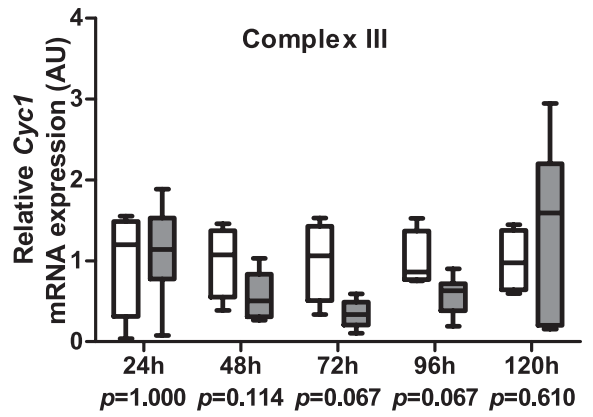

$\mathbf{B}$

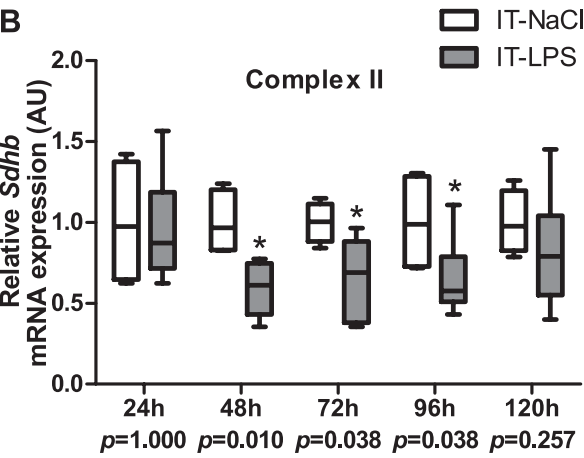

D

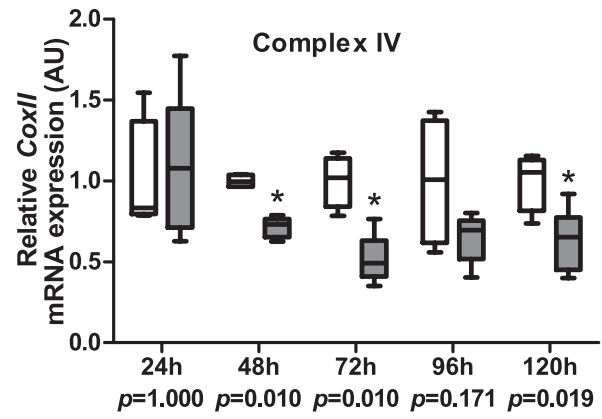

$\triangle$ Figure 7: OXPHOS subunit mRNA expression in $m$. gastrocnemius in response to IT-LPS. mRNA expression levels of the following OXPHOS subunits in $\mathrm{m}$. gastrocnemius are depicted; Ndufb3 of complex I (A), Sdhb of complex II (B), Cyc1 of complex III (C), and mt-Co2 of complex IV (D). Data is presented as box plots indicating median and interquartile range, with whiskers indicating min and max. $p$-value and significance is depicted between groups within each time-point $* p<0.05$.

Figure 8: mRNA and protein expression of mitochondrial fission and fusion markers in m. gastrocnemius in response to IT-LPS. Fission related mRNA expression of Dmn1/ (A), Fis1 (B), $M \operatorname{tfp} 1(\mathbf{C})$, and protein expression of DMN1L (D) in $\mathrm{m}$. gastrocnemius are depicted. Fusion related mRNA expression of $M f n 1(\mathbf{E})$, and $M f n 2(\mathbf{F})$, and Opa1 (G) in $m$. gastrocnemius are depicted. A representative immunoblot and a representative part of Ponceau $S$ staining are shown, with adjusted contrast equally applied to whole photograph $(\mathbf{H})$. Samples were equally divided over multiple gels which were derived and processed in parallel. Data is presented as box plots indicating median and interquartile range, with whiskers indicating min and max. $p$-value and significance is depicted between groups within each time-point * $p<0.05$. 
A

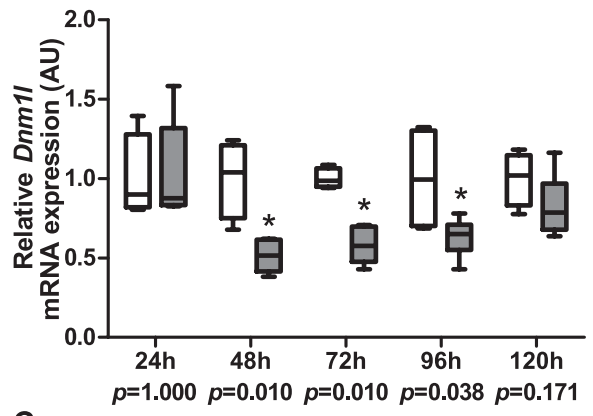

C

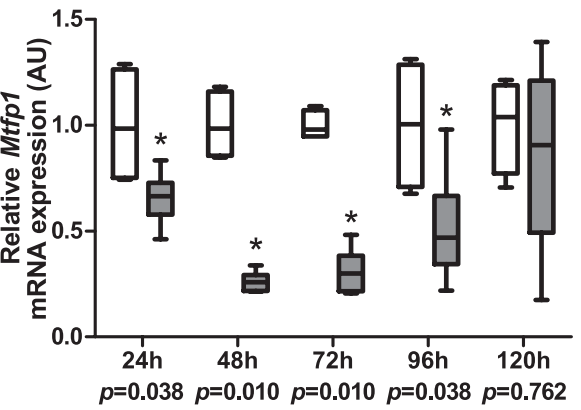

E

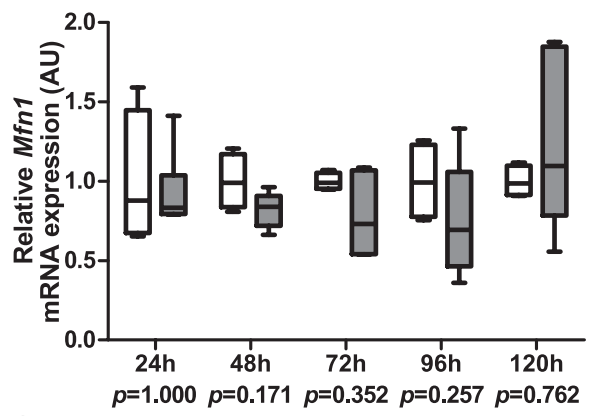

G

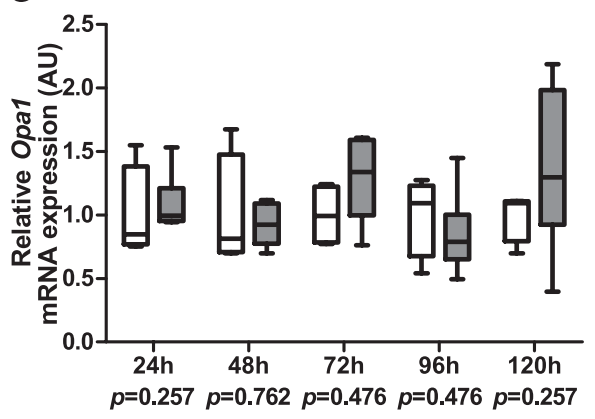

B $\square$ IT-NaCl

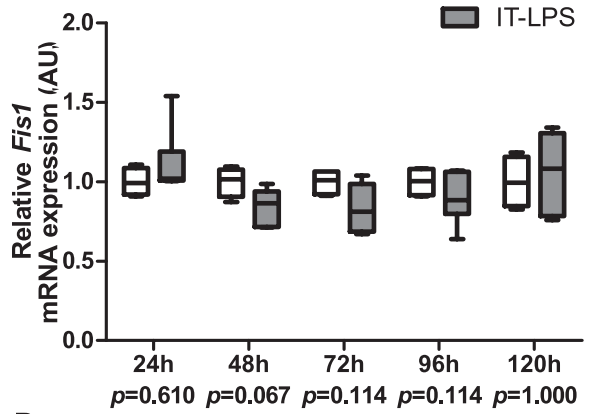

D

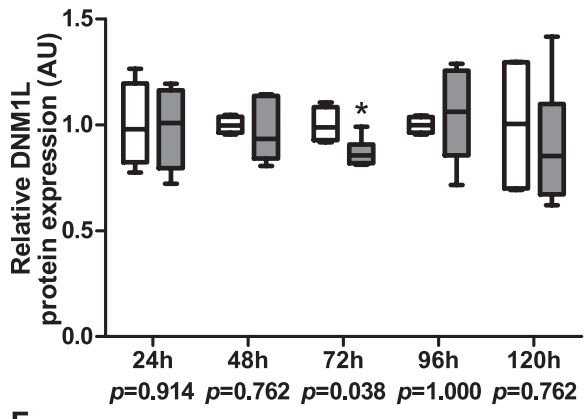

$\mathbf{F}$

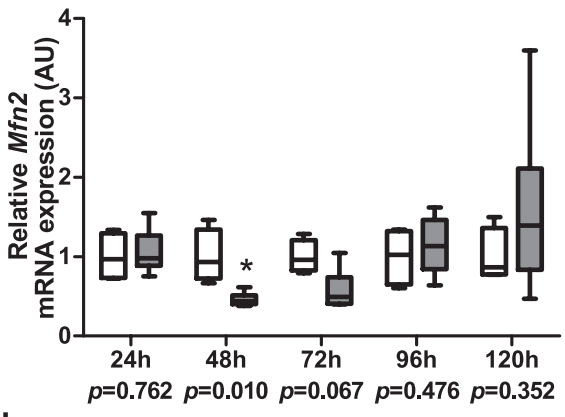

H

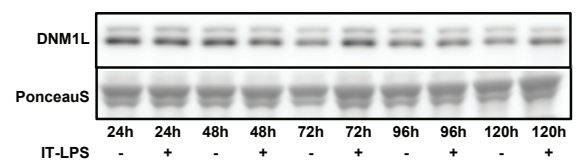


Chapter 4

A

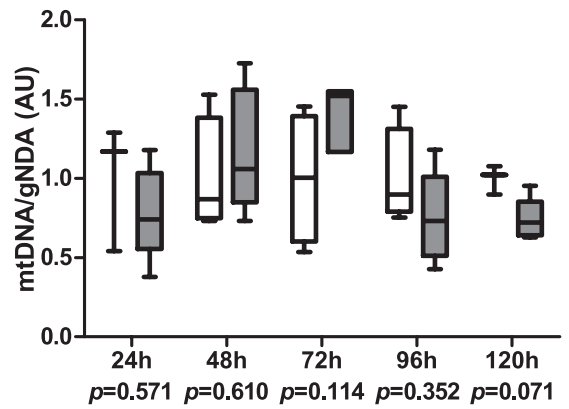

C

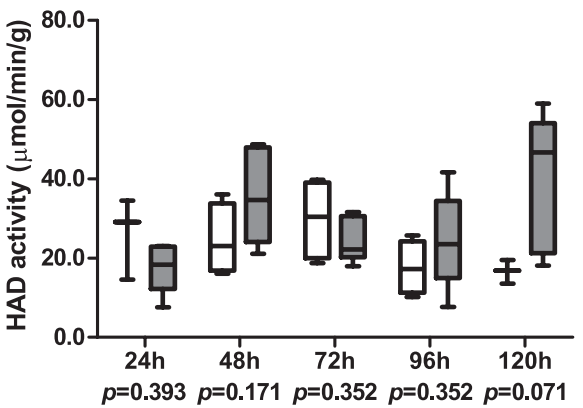

E

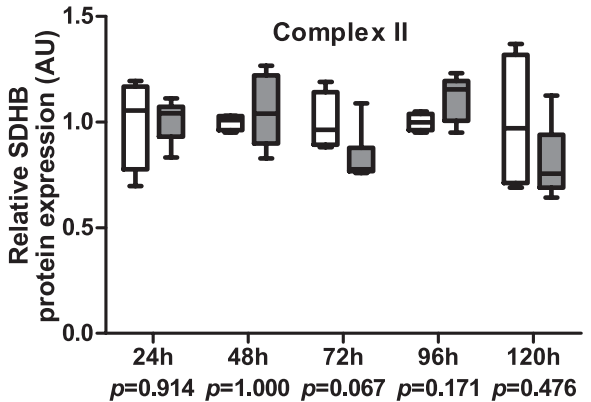

G

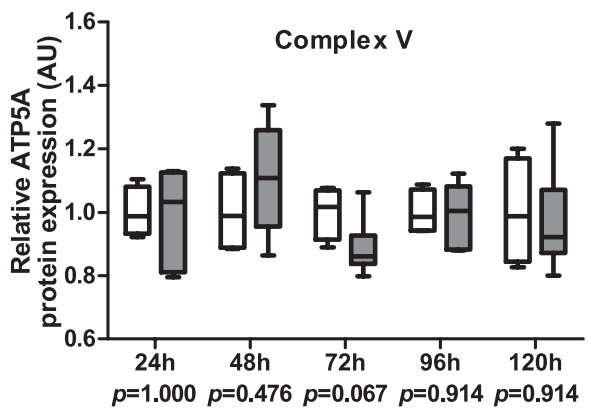

B

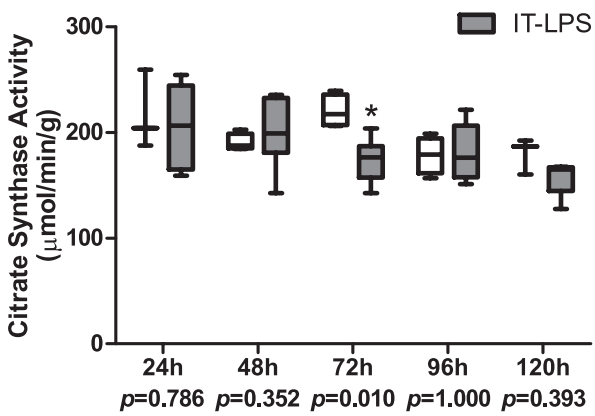

D

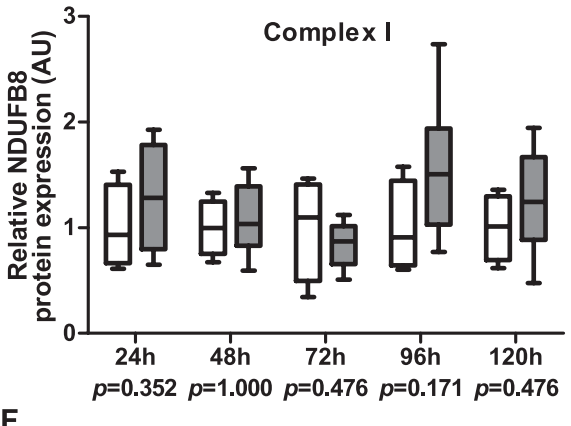

F

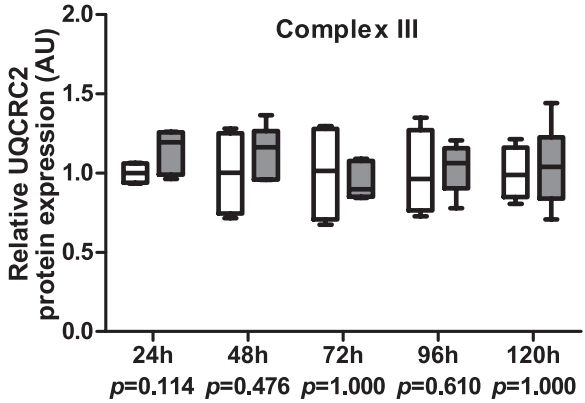

H

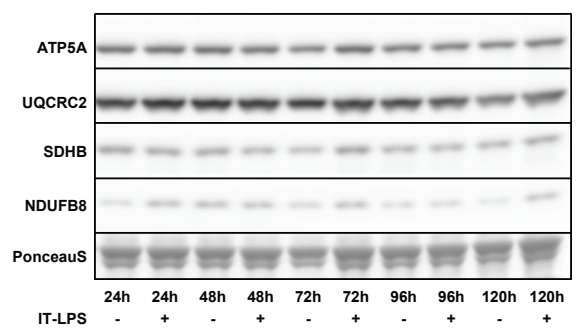

78 
Figure 9: Expression and activity of key mitochondrial constituents in $m$. gastrocnemius in response to IT-LPS. mtDNA corrected for gDNA in m. gastrocnemius is depicted (A). CS (B) and HADH (C) activity in $m$. gastrocnemius are depicted. Protein expression of the following OXPHOS subunits in m. gastrocnemius is depicted; NDUFB8 of complex I (D), SDHB of complex II (E), UQCRC2 of complex III (F), and ATP5A of complex V (G). Representative immunoblots and a representative part of Ponceau Sstaining are shown, with adjusted contrast equally applied to whole photograph $(\mathbf{H})$. Samples were equally divided over multiple gels which were derived and processed in parallel. Data is presented as box plots indicating median and interquartile range, with whiskers indicating min and max. $p$-value and significance is depicted between groups within each time-point ${ }^{*} p<0.05$.

levels were unaltered (Fig. $8 \mathrm{~A}-\mathrm{C}$ ). In addition, in line with mRNA expression levels, protein levels of DNM1L were significantly lower $72 \mathrm{~h}$ post instillation in the IT-LPS mice (Fig. 8 D). With regard to mediators of mitochondrial fusion, only Mfn2 transcript levels were lower $48 \mathrm{~h}$ post instillation in the IT-LPS mice (Fig. $8 \mathrm{E}-\mathrm{F}$ ), while no differences were found for both $M f n 1$ and mitochondrial dynamin like GTPase (Opa1) (Fig. $8 \mathrm{E}-\mathrm{G}$ ).

Mitochondrial content in skeletal muscle in response to IT-LPS. A secondary objective of this study was to explore whether the abovementioned alterations in abundance of molecular signalling constituents of mitophagy and mitochondrial biogenesis in muscle in response to an acutely-administered single bolus of IT-LPS were sufficient to induce changes in skeletal muscle mitochondrial content within the timeframe of the study. Therefore, we measured mtDNA copy number, protein levels of subunits of 4 mitochondrial OXPHOS complexes as well as activity levels of CS and HADH, 2 key mitochondrial enzymes respectively involved in the Krebs cycle and fatty acid $\beta$-oxidation.

mtDNA copy number tended to be lower $120 \mathrm{~h}$ post instillation in mice subjected to IT-LPS compared with IT-NaCl (Fig. 9 A). CS enzyme activity was lower $72 \mathrm{~h}$ post instillation, while no significant differences for HADH enzyme activity were found in mice subjected to IT-LPS compared with controls (Fig. 9 B-C). Protein levels of subunits from OXPHOS complex II and $V$ tended to be lower in the LPS group $72 \mathrm{~h}$ post instillation (Fig. $9 \mathrm{E}-\mathrm{G}$ ).

\section{Discussion}

As described previously by our group, intra-tracheal instillation of LPS is a well-established model of pulmonary-induced systemic inflammation (23). The inflammatory response in the IT-LPS model has been extensively characterized, with the presence of prominent pulmonary inflammation, as identified by inflammatory cell influx and activation of inflammatory gene expression, and 
systemic inflammation evidenced by increased presence of TNF- $\alpha$, IL-1 $\alpha$, IL-1 $\beta$, IL-6, CXCL1, RANTES and G-CSF in the circulation (23-25). The IT-LPS induced systemic cytokine profile has a delayed response and smaller magnitude compared with mice subjected to i.p. LPS $(23,30)$. Moreover, activation of inflammatory NF-KB signalling in muscle has been described in the IT-LPS model, which is in line with increased levels of inflammatory gene expression in our current study (23).

We and others have previously demonstrated that IT-LPS instillation adversely impacts the peripheral musculature as evidenced by the activation of muscle catabolic signalling and atrophy of the tissue. Our current study now shows, for the first time, that a single intra-tracheally instilled bolus of LPS results in modulation of mitophagy-associated signalling in peripheral skeletal muscle. These changes were associated with an initial decrease of transcript abundance of PPARGC1B, PPARA and ESRRA, decreased transcript abundance of several OXPHOS complexes, and with reductions in transcript and protein levels of several proteins essentially involved in mitochondrial fission events.

Whether inflammation impacts mitophagy or its key regulatory proteins in skeletal muscle in vivo is largely unexplored. In cardiac muscle however, i.p. LPS injection was shown to increase mitophagy, characterized by a rapid induction of protein levels of BNIP3 and its binding partner LC3B, and a delayed increase of BNIP3L protein and mRNA expression levels of its binding partner GABARAPL1 (31). Others reported a skeletal muscle-based induction of general autophagy upon i.p. LPS injection, which included an induction in mRNA expression of mitophagy receptor BNIP3 (32). Although BNIP3 and BNIP3L have also been reported as mediators of cell death (33), our collective findings in combination with the existing literature suggest that LPS-induced (systemic) inflammation can indeed modulate mitophagy-associated processes in peripheral tissues such as the heart and skeletal muscle thereby potentially impacting mitochondrial homeostasis in these organs.

In contrast to BNIP3 and BNIP3L activation, which has been reported to be transcriptionallyregulated (34), FUNDC1 activation, anditssubsequentmitophagymediated breakdown, is mainly determined by post-translational modifications (e.g. phosphorylation) (35-38). In line with this, we report no profound alter1ations in Fundc1 transcript levels while FUNDC1 protein abundance decreased in response to LPS. However, recent literature also reports that during acute stress, FUNDC1 can rapidly be degraded by mitophagy-independent breakdown to prevent the tissue from excess mitophagy (39), which makes interpretation of our FUNDC1-related data speculative. Although FUNDC1- 
mediated mitophagy has extensively been studied in several mammalian cell types during hypoxia $(14,37)$, its activation in skeletal muscle in response to inflammation has not been reported in vivo thus far.

Although the abovementioned alterations in mitophagy signalling were not associated by changes in PPARGC1A, NRF1 or TFAM transcript or protein expression, they coincided with prominent reductions in the transcript levels of PPARGC1B, PPARA and ESRRA. PPARGC1B has been described to play an important role in the skeletal muscle oxidative phenotype (40), where it promotes transcription of mitochondrial genes when linked to transcription factors like NRFs and ESRRA or fatty acid oxidation when linked to PPARs (41). In line with the decreased transcript levels of PPARGC1B and ESRRA, we observed decreased transcript, but not protein, abundance of several nuclear- and mitochondrial-encoded OPXHOS subunits. Although we did not find profound changes in the skeletal muscle oxidative phenotype in our mice, others reported that i.p. LPS injection resulted in the decrease of the reduced respiratory control ratio (RCR), and ADP- and FCCP-stimulated respiration in isolated mitochondria from skeletal muscle of mice (7). Whether the alterations in mitophagy and mitochondrial biogenesis signalling in the initial phase of IT-LPS-induced inflammation represent a coordinated response remains to be established.

As we previously demonstrated a direct causal relationship between activation of NF-KB signalling and impairment of oxidative metabolism and the regulation thereof by the PPARGC1 network in cultured muscle cells $(6,9)$, it is feasible that IT-LPS-induced activation of muscle NF-KB signalling, which has been described by our group previously (23), is implicated in the impairments in the constituents controlling mitochondrial metabolism that we observed in the initial phase post IT-LPS. Also, autophagy-related gene-expression was found to be NF-KB-dependent in both IT-LPS and i.p. LPS inflammation models, while BNIP3 gene-expression was not $(23,32)$. Although NF-KB dependency was not examined in the current study, the increase of BNIP3, BNIP3L levels, and to a lesser extent the decrease of FUNDC1, together with the increase of their supporting autophagy-binding partners LC3B and GABARAPL1, coincides with the previously reported increased skeletal muscle NF-KB signalling in response to IT-LPS instillation suggesting that these processes may well be related (23).

Intriguingly, in recent years mitophagy has been identified, not only as an essential mechanism of mitochondrial clearance but also as a key process involved in mitochondrial biogenesis (22). For example, whereas receptormediated mitophagy (mediated by e.g. BNIP3, BNIP3L) seemed to be mainly induced during conditions of mitochondrial clearance, PINK1/PARK2- 
based mitophagy was found to be initiated during biogenesis and remodelling of mitochondria in cardiac muscle following cardiac stress $(22,42)$. In line with this notion, knock-out of PARK2 protein in cardiac muscle of mice subjected to i.p. LPS-induced sepsis preferentially prevented recovery of mitochondria rather than initial mitochondrial clearance (31). Based on available evidence, PINK1/PARK2 can regulate mitochondrial biogenesis by modulating Parkin-interacting substrate (PARIS), an inhibitor of PPARGC1A and its target genes (43). Although we report only a marginal increase in PARK2 protein levels at $96 \mathrm{~h}$ post IT-LPS, a previous study showed increased PARK2 in isolated mitochondria of heart tissue $24 \mathrm{~h}$ after i.p. LPS (31). Since we might have missed a re-localization of PARK2 from the cytoplasm to the mitochondria while the absolute PARK2 quantity remained unaltered, it remains to be established whether or not activation of the PINK1/PARK2 pathway is involved in the recovery phase at later time-points after IT-LPS. Although we did not find altered total protein abundance of PPARGC1A, TFAM or NRF1, alterations in subcellular distribution or post-translational modification of these proteins (44) may well have contributed to the recovery of transcript levels of OXPHOS subunits or constituents of the PPARGC1 signalling network.

Mitochondrial fission and fusion events are of key importance in maintaining mitochondrial homeostasis and are tightly linked to both mitochondrial biogenesis and mitophagy (22). Indeed, PINK1, PARK2 as well as FUNDC1 are heavily involved in regulating fusion and fission events (15, 35, 45-47). In addition to this interplay with mitophagy, mitochondrial fission/fusion also has tight links with mitochondrial biogenesis. For example, it was shown that transcription of Mfn2 is controlled by PPARGC1 and ESRRA $(48,49)$. In line with this, decreased transcript abundance of PPARGC1B and ESRRA in our study coincided with decreased mRNA expression of MFN2. Although we did not measure actual fission or fusion, our data shows a clear modulation of the transcriptional regulation of these processes.

The abovementioned alterations in abundance of molecular signalling constituents of mitophagy and mitochondrial biogenesis were not associated with reductions in parameters of mitochondrial content, likely due to the acute nature of the model precluding robust changes in mitochondrial content.

\section{Limitations of the study}

Although we report data on a comprehensive set of proteins involved in the execution and regulation of mitophagy, autophagy, mitochondrial biogenesis, and mitochondrial dynamics measured in an extensive time-course after 
instillation of IT-LPS, we are aware that our study has some limitations. First, we quantified abundance of many key players heavily involved in, and indicative of mitophagy initiation but no actual mitophagy or autophagy flux was measured in our samples. Therefore, the increased protein abundance of the mitophagy and autophagy-related proteins might also be the result of impaired breakdown instead of increased synthesis. Moreover, we describe associations between changes in the processes of autophagy/mitophagy and mitochondrial biogenesis, but the causality of these associations remains unclear in our study. In addition to the known decrease in physical activity, it has been shown that food intake is reduced in this model (28). As decreased physical activity and food intake have both been shown to be able to modulate mitophagy and mitochondrial biogenesis in muscle, a potential contribution of these aspects to the changes we observed in muscle of IT-LPS-instilled animals cannot be discarded. More specifically, with regard to starvation in this model, it has been shown previously that reduced food intake accounts for $60 \%$ of the loss in body weight and $55 \%$ of the loss of muscle weight $24 \mathrm{~h}$ after IT-LPS instillation. Additionally, this study also demonstrated that IT-LPS-induced increased mRNA expression of BNIP3 was not, and that of GABARAPL was only partly, due to activation of muscle NF-KB signalling in this model (23). Therefore, as we did not include a pair-fed group, the relative contributions of starvation and inflammation to the activation of the autophagy/mitophagy pathways in peripheral muscle cannot be discerned in the current study. Lastly, although we observed significant differences in expression levels and abundance of several proteins essentially involved in mitophagy and mitochondrial biogenesis, the relatively small group sizes in the current study may have precluded statistically significant differences in some of the proteins we investigated (e.g. FUNDC1 and BNIP3L protein abundance).

Despite these limitations, this is the first study reporting a comprehensive overview of changes in key proteins controlling mitophagy, mitochondrial biogenesis and mitochondrial dynamics which suggest modulation of these processes in peripheral muscle in vivo in response to IT-LPS instillation, which hasn't been described in literature so far.

In conclusion, IT-LPS instillation resulted in molecular patterns indicative of activation of mitophagy in skeletal muscle. This coincided with modulation of mRNA transcript abundance of genes involved in mitochondrial fusion and fission as well as an initial decrease and subsequent recovery of transcript levels of key proteins involved in the molecular regulation of mitochondrial biogenesis. Given the interconnectivity of molecular pathways regulating the processes of mitophagy and mitochondrial biogenesis, the timeframe of the alterations 
observed in these pathways might well be illustrative of a coordinated physiological response of skeletal musculature to inflammation, induced by acute in vivo LPS administration. However, no solid differences in markers for mitochondrial content were found. Therefore, these data suggest that one bolus of IT-LPS results in the temporal modulation of mitochondrial clearance and biogenesis signalling, but is insufficient to result in reductions of indices of mitochondrial content.

\section{Acknowledgements}

We thank Douglas Gray for providing the transgenic murine strain used.

\section{Funding}

This work was supported by the Nutrim Graduate Programme; and the Netherland's Lung Foundation (grant number 5.2.13.067JO). 


\section{References}

1. Romanello V, Sandri M. Mitochondrial Quality Control and Muscle Mass Maintenance. Frontiers in physiology. 2015;6:422. PubMed PMID: 26793123. Pubmed Central PMCID: 4709858.

2. Kinugawa S, Takada S, Matsushima S, Okita K, Tsutsui H. Skeletal Muscle Abnormalities in Heart Failure. International heart journal. 2015;56(5):475-84. PubMed PMID: 26346520.

3. Maltais F, Decramer M, Casaburi R, Barreiro E, Burelle Y, Debigare R, et al. An official American Thoracic Society/European Respiratory Society statement: update on limb muscle dysfunction in chronic obstructive pulmonary disease. American journal of respiratory and critical care medicine. 2014 May 1;189(9): e15-62. PubMed PMID: 24787074. Pubmed Central PMCID: 4098112.

4. Molina AJ, Bharadwaj MS, Van Horn C, Nicklas BJ, Lyles MF, Eggebeen J, et al. Skeletal Muscle Mitochondrial Content, Oxidative Capacity, and Mfn2 Expression Are Reduced in Older Patients With Heart Failure and Preserved Ejection Fraction and Are Related to Exercise Intolerance. JACC Heart failure. 2016 May 3. PubMed PMID: 27179829.

5. Lavine KJ, Sierra OL. Skeletal muscle inflammation and atrophy in heart failure. Heart failure reviews. 2017 Mar;22(2):179-89. PubMed PMID: 28091823. Pubmed Central PMCID: 5352459.

6. Remels AH, Gosker HR, Langen RC, Polkey M, Sliwinski P, Galdiz J, et al. Classical NF-kappaB activation impairs skeletal muscle oxidative phenotype by reducing IKK-alpha expression. Biochimica et biophysica acta. 2014 Feb;1842(2):175-85. PubMed PMID: 24215713.

7. Frisard MI, Wu Y, McMillan RP, Voelker KA, Wahlberg KA, Anderson AS, et al. Low levels of lipopolysaccharide modulate mitochondrial oxygen consumption in skeletal muscle. Metabolism: clinical and experimental. 2015 Mar;64(3):416-27. PubMed PMID: 25528444. Pubmed Central PMCID: 4501015.

8. Tanaka K, Tanaka M, Takegaki J, Fujino H. Preventive effects of electrical stimulation on inflammation-induced muscle mitochondrial dysfunction. Acta histochemica. 2016 May 18. PubMed PMID: 27209425.

9. Remels AH, Gosker HR, Bakker J, Guttridge DC, Schols AM, Langen RC. Regulation of skeletal muscle oxidative phenotype by classical NF-kappaB signalling. Biochimica et biophysica acta. 2013 Aug;1832(8):1313-25. PubMed PMID: 23563317.

10. Gomes LC, Scorrano L. Mitochondrial morphology in mitophagy and macroautophagy. Biochimica et biophysica acta. 2013 Jan;1833(1):205-12. PubMed PMID: 22406072.

11. Wei H, Liu L, Chen Q. Selective removal of mitochondria via mitophagy: distinct pathways for different mitochondrial stresses. Biochimica et biophysica acta. 2015 Oct;1853(10 Pt B):2784-90. PubMed PMID: 25840011.

12. Schwarten M, Mohrluder J, Ma P, Stoldt M, Thielmann Y, Stangler T, et al. Nix directly binds to GABARAP: a possible crosstalk between apoptosis and autophagy. Autophagy. 2009 Jul;5(5):690-8. PubMed PMID: 19363302. 
13. Hanna RA, Quinsay MN, Orogo AM, Giang K, Rikka S, Gustafsson AB. Microtubuleassociated protein 1 light chain 3 (LC3) interacts with Bnip3 protein to selectively remove endoplasmic reticulum and mitochondria via autophagy. The Journal of biological chemistry. 2012 Jun 1;287(23):19094-104. PubMed PMID: 22505714. Pubmed Central PMCID: 3365942.

14. Yamaguchi O, Murakawa T, Nishida K, Otsu K. Receptor-mediated mitophagy. Journal of molecular and cellular cardiology. 2016 Mar 25(95):50-6. PubMed PMID: 27021519.

15. Gegg ME, Cooper JM, Chau KY, Rojo M, Schapira AH, Taanman JW. Mitofusin 1 and mitofusin 2 are ubiquitinated in a PINK1/parkin-dependent manner upon induction of mitophagy. Human molecular genetics. 2010 Dec 15;19(24):4861-70. PubMed PMID: 20871098. Pubmed Central PMCID: 3583518.

16. Eiyama A, Okamoto K. PINK1/Parkin-mediated mitophagy in mammalian cells. Current opinion in cell biology. 2015 Apr;33:95-101. PubMed PMID: 25697963.

17. Lazarou M, Sliter DA, Kane LA, Sarraf SA, Wang C, Burman JL, et al. The ubiquitin kinase PINK1 recruits autophagy receptors to induce mitophagy. Nature. 2015 Aug 20;524(7565):309-14. PubMed PMID: 26266977.

18. Correia JC, Ferreira DM, Ruas JL. Intercellular: local and systemic actions of skeletal muscle PGC-1s. Trends in endocrinology and metabolism: TEM. 2015 Jun;26(6): 305-14. PubMed PMID: 25934582.

19. Lin J, Wu H, Tarr PT, Zhang CY, Wu Z, Boss O, et al. Transcriptional co-activator PGC-1 alpha drives the formation of slow-twitch muscle fibres. Nature. 2002 Aug 15;418(6899):797-801. PubMed PMID: 12181572.

20. Erlich AT, Tryon LD, Crilly MJ, Memme JM, Moosavi ZSM, Oliveira AN, et al. Function of specialized regulatory proteins and signaling pathways in exercise-induced muscle mitochondrial biogenesis. Integrative medicine research. 2016 Sep;5(3): 187-97. PubMed PMID: 28462117. Pubmed Central PMCID: 5390460.

21. Remels AH, Schrauwen P, Broekhuizen R, Willems J, Kersten S, Gosker HR, et al. Peroxisome proliferator-activated receptor expression is reduced in skeletal muscle in COPD. The European respiratory journal. 2007 Aug;30(2):245-52. PubMed PMID: 17459894.

22. Palikaras K, Tavernarakis N. Mitochondrial homeostasis: The interplay between mitophagy and mitochondrial biogenesis. Experimental gerontology. 2014 Jan 28(56):182-8. PubMed PMID: 24486129.

23. Langen RC, Haegens A, Vernooy JH, Wouters EF, de Winther MP, Carlsen $\mathrm{H}$, et al. NF-kappaB activation is required for the transition of pulmonary inflammation to muscle atrophy. American journal of respiratory cell and molecular biology. 2012 Sep;47(3):288-97. PubMed PMID: 22538866.

24. Haegens A, Heeringa P, van Suylen RJ, Steele C, Aratani Y, O'Donoghue RJ, et al. Myeloperoxidase deficiency attenuates lipopolysaccharide-induced acute lung inflammation and subsequent cytokine and chemokine production. Journal of immunology. 2009 Jun 15;182(12):7990-6. PubMed PMID: 19494324.

25. Gamble L, Bagby GJ, Quinton LJ, Happel KI, Mizgerd JP, Zhang P, et al. The systemic and pulmonary LPS binding protein response to intratracheal lipopolysaccharide. Shock. 2009 Feb;31(2):212-7. PubMed PMID: 18520704. 
26. Grundy D. Principles and standards for reporting animal experiments in The Journal of Physiology and Experimental Physiology. The Journal of physiology. 2015 Jun 15;593(12):2547-9. PubMed PMID: 26095019. Pubmed Central PMCID: 4500341.

27. Tsirigotis $M$, Thurig S, Dube M, Vanderhyden BC, Zhang M, Gray DA. Analysis of ubiquitination in vivo using a transgenic mouse model. BioTechniques. 2001 Jul;31(1):120-6, 8, 30. PubMed PMID: 11464505.

28. Ceelen JJM, Schols A, Thielen NGM, Haegens A, Gray DA, Kelders M, et al. Pulmonary inflammation-induced loss and subsequent recovery of skeletal muscle mass require functional poly-ubiquitin conjugation. Respiratory research. 2018 May 2;19(1):80. PubMed PMID: 29720191. Pubmed Central PMCID: 5932886.

29. Slot IG, Schols AM, de Theije CC, Snepvangers FJ, Gosker HR. Alterations in Skeletal Muscle Oxidative Phenotype in Mice Exposed to 3 Weeks of Normobaric Hypoxia. Journal of cellular physiology. 2016 Feb;231(2):377-92. PubMed PMID: 26129845.

30. Juskewitch JE, Knudsen BE, Platt JL, Nath KA, Knutson KL, Brunn GJ, et al. LPS-induced murine systemic inflammation is driven by parenchymal cell activation and exclusively predicted by early MCP-1 plasma levels. The American journal of pathology. 2012 Jan;180(1):32-40. PubMed PMID: 22067909. Pubmed Central PMCID: 3338351.

31. Piquereau J, Godin R, Deschenes S, Bessi VL, Mofarrahi M, Hussain SN, et al. Protective role of PARK2/Parkin in sepsis-induced cardiac contractile and mitochondrial dysfunction. Autophagy. 2013 Nov 1;9(11):1837-51. PubMed PMID: 24121678.

32. Mofarrahi M, Sigala I, Guo Y, Godin R, Davis EC, Petrof B, et al. Autophagy and skeletal muscles in sepsis. PloS one. 2012;7(10):e47265. PubMed PMID: 23056618. Pubmed Central PMCID: 3467208.

33. Zhang J, Ney PA. Role of BNIP3 and NIX in cell death, autophagy, and mitophagy. Cell death and differentiation. 2009 Jul;16(7):939-46. PubMed PMID: 19229244. Pubmed Central PMCID: 2768230.

34. Burton TR, Gibson SB. The role of Bcl-2 family member BNIP3 in cell death and disease: NIPping at the heels of cell death. Cell death and differentiation. 2009 Apr;16(4):515-23. PubMed PMID: 19136941. Pubmed Central PMCID: 3158804.

35. Chen M, Chen Z, Wang Y, Tan Z, Zhu C, Li Y, et al. Mitophagy receptor FUNDC1 regulates mitochondrial dynamics and mitophagy. Autophagy. 2016 Apr 2;12(4):689-702. PubMed PMID: 27050458. Pubmed Central PMCID: 4836026.

36. Wu W, Tian W, Hu Z, Chen G, Huang L, Li W, et al. ULK1 translocates to mitochondria and phosphorylates FUNDC1 to regulate mitophagy. EMBO reports. 2014 May;15(5):566-75. PubMed PMID: 24671035. Pubmed Central PMCID: 4210082.

37. Liu L, Feng $D$, Chen $G$, Chen $M$, Zheng $Q$, Song $P$, et al. Mitochondrial outermembrane protein FUNDC1 mediates hypoxia-induced mitophagy in mammalian cells. Nature cell biology. 2012 Feb;14(2):177-85. PubMed PMID: 22267086.

38. Lv M, Wang C, Li F, Peng J, Wen B, Gong Q, et al. Structural insights into the recognition of phosphorylated FUNDC1 by LC3B in mitophagy. Protein \& cell. 2017 Jan;8(1):25-38. PubMed PMID: 27757847. Pubmed Central PMCID: 5233613. 
39. Chen Z, Liu L, Cheng Q, Li Y, Wu H, Zhang W, et al. Mitochondrial E3 ligase MARCH5 regulates FUNDC1 to fine-tune hypoxic mitophagy. EMBO reports. 2017 Mar;18(3):495-509. PubMed PMID: 28104734. Pubmed Central PMCID: 5331199.

40. Arany Z. PGC-1 coactivators and skeletal muscle adaptations in health and disease. Current opinion in genetics \& development. 2008 Oct;18(5):426-34. PubMed PMID: 18782618. Pubmed Central PMCID: 2629557.

41. Lin J, Handschin C, Spiegelman BM. Metabolic control through the PGC-1 family of transcription coactivators. Cell metabolism. 2005 Jun;1(6):361-70. PubMed PMID: 16054085.

42. Dorn GW, 2nd. Central Parkin: The evolving role of Parkin in the heart. Biochimica et biophysica acta. 2016 Aug;1857(8):1307-12. PubMed PMID: 26992930. Pubmed Central PMCID: 4893897.

43. Shin JH, Ko HS, Kang H, Lee $\mathrm{Y}$, Lee $\mathrm{YI}$, Pletinkova O, et al. PARIS (ZNF746) repression of PGC-1alpha contributes to neurodegeneration in Parkinson's disease. Cell. 2011 Mar 4;144(5):689-702. PubMed PMID: 21376232. Pubmed Central PMCID: 3063894.

44. Fernandez-Marcos PJ, Auwerx J. Regulation of PGC-1alpha, a nodal regulator of mitochondrial biogenesis. The American journal of clinical nutrition. 2011 Apr;93(4):884S-90. PubMed PMID: 21289221. Pubmed Central PMCID: 3057551.

45. Poole AC, Thomas RE, Yu S, Vincow ES, Pallanck L. The mitochondrial fusionpromoting factor mitofusin is a substrate of the PINK1/parkin pathway. PloS one. 2010;5(4):e10054. PubMed PMID: 20383334. Pubmed Central PMCID: 2850930.

46. Chen Y, Dorn GW, 2nd. PINK1-phosphorylated mitofusin 2 is a Parkin receptor for culling damaged mitochondria. Science. 2013 Apr 26;340(6131):471-5. PubMed PMID: 23620051. Pubmed Central PMCID: 3774525.

47. Pryde KR, Smith HL, Chau KY, Schapira AH. PINK1 disables the anti-fission machinery to segregate damaged mitochondria for mitophagy. The Journal of cell biology. 2016 Apr 25;213(2):163-71. PubMed PMID: 27091447.

48. Liesa M, Borda-d'Agua B, Medina-Gomez G, Lelliott CJ, Paz JC, Rojo M, et al. Mitochondrial fusion is increased by the nuclear coactivator PGC-1beta. PloS one. 2008;3(10):e3613. PubMed PMID: 18974884. Pubmed Central PMCID: 2570954.

49. Soriano FX, Liesa M, Bach D, Chan DC, Palacin M, Zorzano A. Evidence for a mitochondrial regulatory pathway defined by peroxisome proliferator-activated receptor-gamma coactivator-1 alpha, estrogen-related receptor-alpha, and mitofusin 2. Diabetes. 2006 Jun;55(6):1783-91. PubMed PMID: 16731843. 


\section{Early-onset alterations in mitophagy and mitochondrial biogenesis signalling during skeletal muscle unloading in mice and men}

P.A. Leermakers, A.E.M. Kneppers, A.M.W.J. Schols, M.C.J.M. Kelders, C.C. de Theije, L.B. Verdijk, L.J.C. van Loon, R.C.J. Langen, H.R. Gosker

Submitted 


\section{Abstract}

Physical inactivity significantly contributes to rapid loss of muscle mass and muscle performance in hospitalized and bed-bound patients. Although, the underlying loss of skeletal muscle mitochondrial content and oxidative capacity has been well-established in rodent and human models for skeletal muscle unloading, the complete molecular mechanism remains unclear. Since mitochondrial content is determined by the balance between mitochondrial biogenesis and mitochondrial breakdown (e.g. by mitochondrial autophagy (mitophagy)), we hypothesized that the unloading-induced loss of muscle mitochondrial quantity is preceded by an increase in mitophagy- and a decrease in mitochondrial biogenesis-signalling during the early stages of muscle unloading. To address this, we analysed a comprehensive set of molecular markers involved in the execution and regulation of mitophagy, autophagy, mitochondrial biogenesis, and mitochondrial dynamics, as well as markers for mitochondrial quantity, in the $m$. gastrocnemius of mice subjected to 0 - and 3-days hind limb suspension, and in biopsies from human $m$. vastus lateralis obtained before and after 7-days of one-leg immobilization. We show that both in mice and men short-term skeletal muscle unloading results in molecular marker patterns indicative of increased receptor-mediated mitophagy and decreased mitochondrial biogenesis, before the loss of mitochondrial content. In conclusion, these results emphasize the early-onset of skeletal muscle disuse-induced mitochondrial remodelling, which may have implications for hospitalized and bed-bound patients, as well as inactive patients with chronic diseases. 


\section{Introduction}

Physical inactivity significantly contributes to rapid loss of muscle mass and muscle performance in hospitalized and bed-bound patients $(1,2)$. Moreover, the loss of skeletal muscle mitochondrial content and oxidative capacity, which are both determinants of muscle performance, has been reported in men exposed to 1 week of strict bed rest, and 2 weeks of one-leg skeletal muscle immobilization $(1,2)$, and has been well-established in different models of both rat and murine skeletal muscle unloading (3-10). However, both data on the molecular regulation of mitochondrial breakdown during the early-phase of muscle unloading in murine models of muscle disuse, and data on the molecular regulation of mitochondrial breakdown in human unloaded subjects remains limited to date.

Since maintenance of a healthy mitochondrial pool requires constant mitochondrial renewal, the total mitochondrial content is determined by the balance between mitochondrial biogenesis and breakdown, of which mitochondrial breakdown primarily occurs through mitophagy (11). Mitochondrial biogenesis (i.e. de novo synthesis of mitochondria) is mainly regulated by the peroxisome proliferator-activated receptor (PPAR) co-activator (PGC) signalling network (12), while mitophagy (i.e. a mitochondria-specific form of autophagy (13)) can be regulated by pathways like the receptor-mediated and mitochondrial-based PTEN-induced putative kinase 1 (PINK1)/Parkin-mediated mitophagy, although crosstalk between these pathways has been shown $(14,15)$. Furthermore, skeletal muscle mitochondria are highly dynamic organelles which constantly undergo fission (i.e. separation of mitochondria) and fusion (i.e. merging of mitochondria) events, of which mitochondrial fission is often considered a prerequisite for mitophagy as it isolates small mitochondria from the network $(15,16)$. Therefore, a net loss of mitochondrial content during muscle inactivity may be driven by modulations in mitochondrial biogenesis, mitophagy, dynamics, or a combination of these.

Mitochondrial biogenesis has been extensively studied in rodent models for skeletal muscle inactivity, and biogenesis markers are generally found to be decreased in these models $(3-8,17,18)$. Moreover, modulations in protein or mRNA expression of mitophagy-related markers have been found in several rodent models of skeletal muscle inactivity (i.e. hind limb immobilization, suspension, and denervation), all performed with at least 7 days of inactivity when the loss in mitochondrial content is already settled $(4,9,10,19)$. Therefore, it is difficult to establish whether these modulations caused the loss of 
mitochondrial content or are simply the result of a renewed balance after the loss of mitochondrial content. Interestingly, mitophagy signalling has not been studied in the early phases of inactivity preceding overt loss of mitochondrial content. Moreover, many studies have reported expression of mitochondrial dynamics markers in rodent models of muscle inactivity, but convincing evidence indicating increased fission is still lacking $(3,4,6-9,18)$. In human studies, it has been shown that expression of key regulators of mitochondrial biogenesis are lower in the skeletal muscle of chronically inactive compared with active subjects (20-22), and that transcript abundance of some autophagy- and mitophagy-related markers was lower in inactive subjects (22). However, there is only very limited data available on the regulation of mitochondrial biogenesis and mitochondrial dynamics during acute muscle disuse (23-25), and none on regulation of mitophagy as far as we know.

Therefore, we hypothesized that an increase of mitophagy- and a decrease of mitochondrial biogenesis-signalling is present during the early stages of muscle unloading, preceding the loss of mitochondrial quantity, in both the murine and human skeletal muscle. To study this, we analysed a comprehensive set of molecular markers involved in the execution and regulation of mitophagy, autophagy, mitochondrial biogenesis, and mitochondrial dynamics, as well as markers for mitochondrial quantity, in a murine and human model of leg muscle unloading.

\section{Materials and methods}

\section{Ethical approval}

Animal procedures were performed in accordance with the European Directive 2010/63/EU guidelines and conform the journal regulations (26). This study was performed under a protocol approved by the Institutional Animal Care Committee of Maastricht University (DEC-2014-101) in accordance to the National Institutes of Health guide for the care and use of Laboratory animals. The human samples were used from a previously performed double-blind, randomized, placebo-controlled intervention trial, approved by the Medical Ethics Committee of the Maastricht University Medical Centre (registered at www.clinicaltrials.gov as NCT01894737) in accordance with the Declaration of Helsinki, with written informed consent obtained from all subjects (27). 


\section{Animal study design}

Male C57BL/6J wild-type mice were generated, housed, and fed as described previously (28), with exception for the paired fed mice, who received a daily feed amount matched to the consumption of the animals subjected to hind limb suspension. Mice were divided in three groups, which were subjected to no experimental procedure $(B L, n=8), 3$ days of muscle unloading by hind limb suspension (HLS, $n=9$ ), or 3 days of paired feeding (PF, n=9). The HLS protocol was performed as previously described $(28,29)$ with the exception that isoflurane (Abbott, Abbott park, U.S.A.) was used as an anaesthetic instead of halothane. In short, the tail of the animals was wrapped in a tail suspension device and connected to a swivel hook to allow circular motility. This hook was attached to a PVC ring, which slid over an iron rod spanning the length of the cage to allow longitudinal motility. The mice were raised just until the hind limbs were unable to touch the ground or sides of the cage. Body weight and feed intake was monitored throughout the experiment. The PF group received the matched amount of feed consumed by the HLS group, to correct for feed intake. After euthanasia by i.p. sodium pentobarbital (115 $\mathrm{mg} / \mathrm{kg}$ ) followed by exsanguination, lower leg muscles were excised, weighed, snap frozen in liquid nitrogen and stored at $-80^{\circ} \mathrm{C}$ until further analysis.

\section{Human study design and muscle biopsy}

The study group consisted of 13 healthy young men, who previously participated in a trial which was primarily designed to test the potential protective effect of creatine supplementation on disuse-induced loss of muscle mass and function, but the outcome was negative. The study design and primary data were previously published (27).

Briefly, subjects were subjected to muscle unloading through 7 days of one-leg immobilization by means of a full leg cast. Vastus lateralis muscle biopsies of the casted leg were taken following an overnight fast at baseline (BL) and after unloading (UL), and were frozen in liquid nitrogen and stored at $-80^{\circ} \mathrm{C}$ until further analysis. Furthermore, muscle mass (cross sectional area; CSA) was assessed by a computed tomography (CT) scan of the upper legs, muscle strength was estimated using a one-repetition maximum (1-RM) knee extension test, and body weight was measured with a digital balance. None of the subjects performed progressive resistance-type exercise training, or took any medication interfering with muscle metabolism in the previous 6 months. Inclusion in the current study was based on the availability of muscle biopsy material before and after the immobilization period. 


\section{Molecular analyses}

To study molecular markers in the murine model, we analysed the $m$. gastrocnemius, since this murine muscle is considered a mixed muscle as in mice type IIA fibres are the most oxidative fibre type and $m$. gastrocnemius consists of $+/-30 \%$ type IIA and type I fibres (30). In both $m$. gastrocnemius and $m$. vastus lateralis biopsies, we quantified protein- and/or gene-expression of mitophagy markers; BCL2/Adenovirus E1B $19 \mathrm{kDa}$ protein-interacting protein 3 (BNIP3), BNIP3-like (BNIP3L), FUN14 domain-containing protein 1 (FUNDC1), mitochondrial-based PTEN-induced putative kinase 1 (PINK1), and Parkin (PARK2), autophagy markers; microtubule associated protein 1A/1B-light chain 3 beta (MAP1LC3B or LC3B), $y$-aminobutiric acid receptor-associated protein-like 1 (GABARAPL1), sequestosome 1 (SQSTM1), optineurin (OPTN), and Calcium Binding And Coiled-Coil Domain 2 (CALCOCO2), mitochondrial biogenesis markers; proliferative activated receptor (PPAR), gamma, coactivator 1 alpha (PPARGC1A) and beta (PPARGC1B), PPAR delta (PPARD), mitochondrial (TFAM), and nuclear respiratory factor 1 (NRF1), mitochondrial quantity markers; NADH:ubiquinone oxidoreductase subunit B8 (NDUFB8), succinate dehydrogenase complex, subunit B (SDHB), ubiquinol cytochrome c reductase core protein 2 (UQCRC2), mitochondrially encoded cytochrome c oxidase I (mt-COI), ATP synthase, $\mathrm{H}+$ transporting, mitochondrial $\mathrm{F} 1$ complex, alpha (ATP5A), and translocase of outer mitochondrial membrane 20 (TOMM20), mitochondrial dynamics markers; Dynamin 1 Like (DNM1L), Mitochondrial fission 1 protein (FIS1), Optic Atrophy 1 (OPA1), and Mitofusins (MFN1/2), as well as enzyme activity of citrate synthase (CS), hydroxy-acyl CoA dehydrogenase (HADH), and phosphofructokinase (PFK). Enzyme activity was measured only in $m$. gastrocnemius due to sample limitations of the human biopsies.

\section{RNA extraction \& quantitative PCR}

M. gastrocnemius was crushed while frozen. RNA was extracted from crushed tissue using the RNeasy (fibrous tissue) kit (Qiagen, Venlo, the Netherlands) according to the manufacturer's protocol, using a 20 gauge needle for homogenization. 200 ng of RNA was used for cDNA synthesis using the tetro cDNA synthesis kit (Bioline, Alphen aan de Rijn, the Netherlands) according to manufacturer's protocol.

M. vastus lateralis biopsies were crushed while frozen. Crushed tissue was lysed and homogenized using TRIzol (Invitrogen, Carlsbad, CA, USA). Total RNA was isolated by phenol/chloroform extraction and subsequent precipitation from the aqueous phase using glycogen-containing isopropanol. $400 \mathrm{ng}$ of RNA was used for cDNA synthesis using the Transcriptor cDNA synthesis kit (Roche, Almere, the Netherlands) according to manufacturer's protocol. 
4.4 $\mu$ l of diluted cDNA was used for quantitative PCR amplification using target and species specific primers (table 1 ) and $2 X$ Sensimix $^{\mathrm{TM}}$ SYBR $^{\circledR}$ \& Fluorescein mix (Bioline, Alphen aan de Rijn, The Netherlands) and were run in a 384 well white opaque plate on a LightCycler 480 system (Roche, Almere, The Netherlands). Melting curves were analysed to verify specificity of the amplification, and relative quantity of the targets was assessed by LinRegPCR software (v2014.8.1). Study-specific reference genes (i.e. murine; Rplp0, Rpl13A, Ppia, human; RPLPO, $B 2 M, P P I A$ (table 1)) were used to calculate a GeNorm correction factor, which was used to normalize expression of the target genes.

\section{Western Blotting}

Crushed $\mathrm{m}$. gastrocnemius tissue was homogenized in 350-400 $\mu \mathrm{IIP}$ lysis buffer (50 mM Tris, 150 mM NaCl, 10\% glycerol, 0.5\% Nonidet P40, 1 mM EDTA, 1 mM Na3VO4, 5 mM NaF, 10 mM $\beta$-glycerophosphate, 1 mM Na4O7P2, 1 mM DTT, $10 \mu \mathrm{g} / \mu \mathrm{l}$ leupeptin, 1\% apropeptin, $1 \mathrm{mM}$ PMSF, pH 7.4) with the Polytron PT 1600 E (Kinematica). Lysates were incubated while rotating for $15 \mathrm{~min}$ at $4{ }^{\circ} \mathrm{C}$, and subsequently centrifuged at $20,000 \times \mathrm{g}$ for $30 \mathrm{~min}$ at $4{ }^{\circ} \mathrm{C}$. Protein

Table 1a: primers (murine)

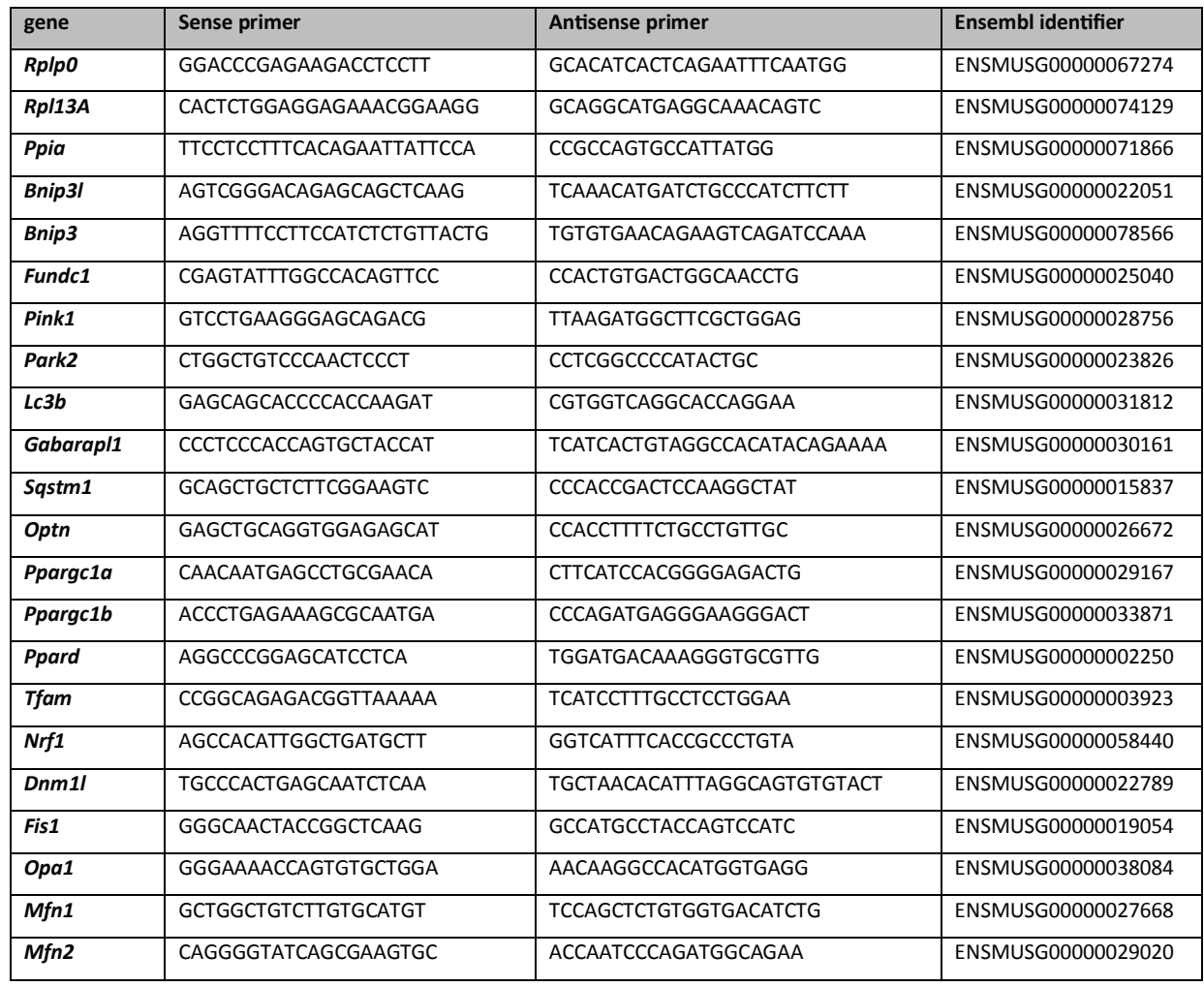


Table 1b: primers (human)

\begin{tabular}{|c|c|c|c|}
\hline gene & Sense primer & Antisense primer & Ensembl identifier \\
\hline RPLPO & TCTACAACCCTGAAGTGCTTGATATC & GCAGACAGACACTGGCAACATT & ENSG00000089157 \\
\hline$B 2 M$ & CATCTGCACTGCCAAGACTGA & TTCATGCCTTCTTTCACTTTGC & ENSG00000196262 \\
\hline PPIA & CTGTGCTCGCGCTACTCTCTCTT & TGAGTAAACCTGAATCTTTGGAGTACGC & ENSG00000166710 \\
\hline BNIP3L & CTGCGAGGAAAATGAGCAGTCTCT & GCCCCCCATTTTTCCCATTG & ENSG00000104765 \\
\hline BNIP3 & AGCGCCCGGGATGCA & CCCGTTCCCATTATTGCTGAA & ENSG00000176171 \\
\hline FUNDC1 & GAAACGAGCGAACAAAGCAG & GCAAAAAGCCTCCCACAAAT & ENSG00000069509 \\
\hline PINK1 & GTCTCGGCCTGTCAGGAGAT & CAAGCGTCTCGTGTCCAAC & ENSG00000158828 \\
\hline PRKN & GGTTTGCCTTCTGCCGGGAATG & CTTTCATCGACTCTGTAGGCCTG & ENSG00000185345 \\
\hline$\angle C 3 B$ & ACCATGCCGTCGGAGAAGAC & TCTCGAATAAGTCGGACATCTTCTACTCT & ENSG00000140941 \\
\hline GABARAPL1 & ATCGGAAAAAGGAAGGAGAAAAGATC & CAGGCACCCTGGCTTTTGG & ENSG00000139112 \\
\hline SQSTM1 & GGTGCACCCCAATGTGATCT & CGCAGACGCTACACAAGTCG & ENSG00000161011 \\
\hline OPTN & AAGGAGCAACTGGCATTGCA & TCTCCATCAAGGACTGCCTG & ENSG00000123240 \\
\hline CALCOCO2 & TCTTTGCCGTATCAAGTACCTACT & GGCATTTCTTGATGGAGAGCG & ENSG00000136436 \\
\hline PPARGC1A & GACCAGTGCTACCTGAGAGAGACTT & GCTCGGCTCGGATTTCCT & ENSG00000109819 \\
\hline PPARGC1B & GGCGCTTTGAAGTGTTTGGTGA & TGATGAAGCCGTACTTCTCGCCT & ENSG00000155846 \\
\hline PPARD & TGACCAAAAAGAAGGCCCGC & GTCGTGGATCACAAAGGGCG & ENSG00000112033 \\
\hline TFAM & GAAAGATTCCAAGAAGCTAAGGGTGATT & TCCAGTTTTCCTTTACAGTCTTCAGCTTTT & ENSG00000108064 \\
\hline NRF1 & GCACCTTTGGAGAATGTGGT & CTGGGATAAATGCCCGAAG & ENSG00000106459 \\
\hline DNM1L & CGACTCATTAAATCATATTTTCTCATTGTCAG & TGCATTACTGCCTTTGGCACACT & ENSG00000087470 \\
\hline FIS1 & CCTGGTGCGGAGCAAGTACAA & TCCTTGCTCCCTTTGGGCAG & ENSG00000214253 \\
\hline OPA1 & TACCAAAGGCATTTTGTAGATTCTGAGTT & GCATGCGCTGTATACGCCAA & ENSG00000198836 \\
\hline MFN1 & CTGAGGATGATTGTTAGCTCCACG & CAGGCGAGCAAAAGTGGTAGC & ENSG00000171109 \\
\hline MFN2 & TGGACCACCAAGGCCAAGGA & TCTCGCTGGCATGCTCCAC & ENSG00000116688 \\
\hline
\end{tabular}

concentrations were determined using the Pierce ${ }^{\mathrm{TM}}$ BCA Protein Assay Kit (Thermo Scientific, Landsmeer, the Netherlands) according to the manufacturer's protocol. Lysate $(1 \mu \mathrm{g} / \mu \mathrm{l})$ was aliquoted in sample buffer $(0.25 \mathrm{M}$ Tris- $\mathrm{HCl}$, $8 \%(\mathrm{w} / \mathrm{v})$ SDS, 40\% (v/v) glycerol, 0.4 M DTT, 0.04\% (w/v) Bromphenol Blue, $\mathrm{pH}$ 6.8) and boiled for $5 \mathrm{~min}$ at $100{ }^{\circ} \mathrm{C}$.

Crushed $m$. vastus lateralis tissue was homogenized in lysis buffer $(20 \mathrm{mM}$ Tris-HCL, 1\% Nonidet P40, 5 mM EDTA, 2 mM Na3VO4, 100 mM NaF, 10 mM Na4P2O7, $10 \mathrm{ug} / \mathrm{ml}$ leupeptin, $10 \mathrm{ug} / \mathrm{ml}$ aprotinin, $3 \mathrm{mM}$ Benzamidine, $1 \mathrm{mM}$ PMSF) using the Ultra-Turrax. Lysates were centrifuged for 10 minutes at $10,000 \mathrm{~g}$ at $4^{\circ} \mathrm{C}$. Protein concentrations were determined using the Pierce ${ }^{\mathrm{TM}} \mathrm{BCA}$ Protein Assay Kit (Thermo Scientific, Landsmeer, the Netherlands) according to the manufacturer's protocol. Lysate $(1 \mu \mathrm{g} / \mu \mathrm{l})$ was aliquoted in sample buffer $(0.35 \mathrm{M}$ Tris-HCL, 10\% (w/v) SDS, 30\% (v/v) glycerol, $0.6 \mathrm{M} \mathrm{DTT,} \mathrm{0.02 \%} \mathrm{bromophenol}$ blue), boiled for 5 minutes at $100{ }^{\circ} \mathrm{C}$. 
$10 \mu \mathrm{g}$ of protein per sample was run through a Criterion XT Precast 4-12 or $12 \%$ Bis-Tris gel (Bio-Rad Laboratories B.V., Veenendaal, the Netherlands) in XT MOPS or MES running buffer (Bio-Rad Laboratories B.V., Veenendaal, the Netherlands), and was subsequently blotted on a Nitrocellulose membrane (Bio-Rad Laboratories B.V., Veenendaal, the Netherlands) by electroblotting. At least two protein ladders were loaded on each gel (Precision Plus Protein ${ }^{\text {TM }}$ All Blue Standards \#161-0373, Bio-Rad Laboratories B.V., Veenendaal, the Netherlands).

Membranes were stained with $0.2 \%$ PonceauS in $1 \%$ acetic (Sigma-Aldrich, Zwijndrecht, the Netherlands) and imaged using the Amersham ${ }^{\text {TM }}$ Imager 600 (GE Healthcare Life Sciences, Eindhoven, the Netherlands) to quantify for total protein content. Subsequently, the membranes were blocked at room temperature (RT) in Tween20Tris-buffered saline (TBST; $20 \mathrm{mM}$ Tris, $137 \mathrm{mM} \mathrm{NaCl}, 0.1 \%$ (vol/vol) Tween 20, pH 7.6) containing 3\% (w/v) nonfat dry milk (Campina, Eindhoven, the Netherlands), washed in TBST, and incubated overnight with primary antibody different protein-specific primary antibodies against: oxidative phosphorylation (OXPHOS) complex subunits (MitoScience LLC Cat\# MS604, RRID:AB_2629281), BNIP3L (Cell Signalling Technology Cat\# 12396, RRID:AB_2688036), BNIP3 (Cell Signalling Technology Cat\# 3769S, RRID:AB_2259284), FUNDC1 (Santa Cruz Biotechnology Cat\# sc-133597, RRID:AB_10609242), PARK2 (Cell Signalling Technology Cat\# 4211, RRID:AB_2159920), LC3B (Cell Signalling Technology Cat\# 2775, RRID:AB_915950), GABARAPL1 (Proteintech Group Cat\# 11010-1-AP, RRID:AB_2294415), SQSTM1 (Cell Signalling Technology Cat\# 5114, RRID:AB_10624872), PPARGC1A (Cell Signalling Technology Cat\# 2178S, RRID:AB_823600), TFAM (Millipore Cat\# DR1071, RRID:AB_10682431), NRF1 (Abcam Cat\# ab55744, RRID:AB_2154534), and DNM1L (Cell Signalling Technology Cat\# 8570, RRID:AB_10950498) all diluted 1:1,000-1:10,000 in TBST with $3 \%(\mathrm{w} / \mathrm{v})$ BSA or non-fat dry milk at $4{ }^{\circ} \mathrm{C}$. Membranes were washed, incubated with a HRP-conjugated secondary antibody (\#BA-9200, \#BA-1000, Vector Laboratories, Amsterdam, the Netherlands), diluted 1:10,000 in $3 \%(\mathrm{w} / \mathrm{v})$ non-fat dry milk in TBST, washed, incubated in Supersignal West PICO or FEMTO Chemiluminescent Substrate (Thermo Scientific, Landsmeer, the Netherlands), and imaged using the Amersham ${ }^{\mathrm{TM}}$ Imager 600. Images were quantified with Image Quant software (GE Healthcare Life Sciences, Eindhoven, the Netherlands). Measured protein quantity was corrected for total protein content.

In the murine model, two separate bands were detected for mitophagy receptor BNIP3L, as previously described (Leermakers, Remels), which were termed BNIP3L (upper band) and BNIP3L-II (lower band). 


\section{Enzyme activity assays}

Crushed $m$. gastrocnemius tissue was homogenized in $240 \mu$ SET buffer (250 mM sucrose, 2 mM EDTA, 10 mM Tris, pH 7.4) using the Mini-BeadBeater (Biospec, Bartlesville, U.S.A.) for $30 \mathrm{sec}$. The solution was snap frozen in liquid nitrogen, defrosted and incubated for $30 \mathrm{~min}$ at $4{ }^{\circ} \mathrm{C}$, and centrifuged at $20,000 \times \mathrm{g}$ for $10 \mathrm{~min}$ at $4{ }^{\circ} \mathrm{C} .12 \mu \mathrm{l} 10 \%(\mathrm{w} / \mathrm{v}) \mathrm{BSA}$ was added to $108 \mu \mathrm{l}$ supernatant (resulting in $1 \%(\mathrm{w} / \mathrm{v}) \mathrm{BSA}$ ), which was stored at $-80{ }^{\circ} \mathrm{C}$. Protein concentration was determined by the Pierce ${ }^{\mathrm{TM}}$ BCA Protein Assay Kit in the remaining supernatant.

Enzymatic assays for CS, HADH, and PFK were performed as previously described (31), and were measured using the Multiscan Spectrum machine (Thermo Scientific, Landsmeer, The Netherlands).

\section{Data presentation and statistics}

Data from the murine study are depicted as mean + SEM in either absolute numbers or in case of semi-quantitative measurements as fold change compared with baseline. Group means were tested for significance using an ANOVA with a Bonferroni correction. General characteristics and body composition from the human study are depicted as absolute numbers or as $\Delta$ unloading-baseline as median and minimum maximum. For all molecular markers, $\Delta$ values of unloading-baseline are depicted as a boxplot with median and interquartile range with whiskers indicating minimum and maximum. To test differences between unloading and baseline measurement, Wilcoxon signed-rank tests were performed. All statistical analyses were performed using IBM SPSS 22 software.
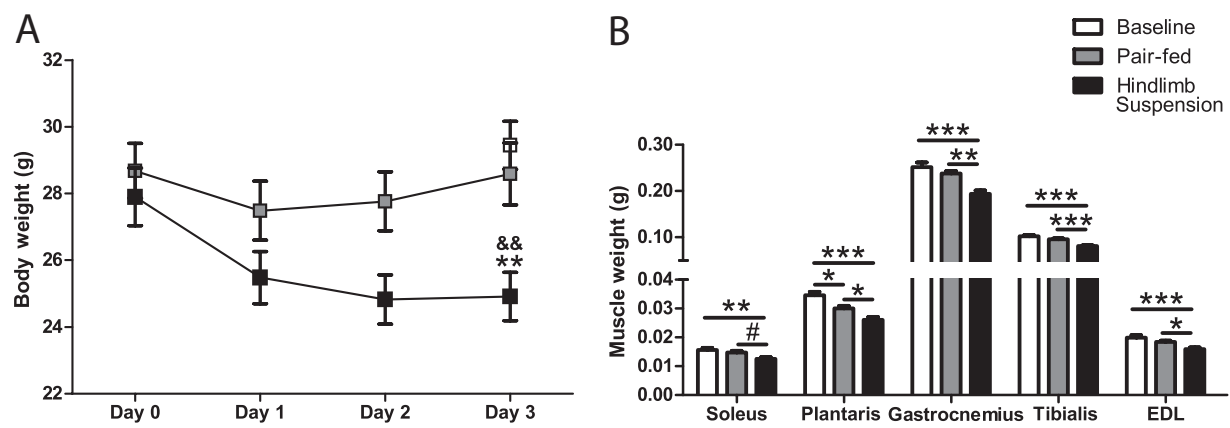

Figure 1: Hind limb suspension-induced body composition changes in mice. Body weight (A) and muscle weights of hind limb muscles (B) are depicted of the murine study. White squares and bars represent $B L$, grey squares and bars represent $P F$, black squares and bars represent HLS. Statistical significance between indicated bars ${ }^{*} p<0.1,{ }^{*} p<0.05,{ }^{* *} p<0.01,{ }^{* * *} p<0.001$, or compared with pair-fed ${ }^{\& \&} p<0.01$ are depicted. 


\section{Results}

\section{Whole body and muscle response to unloading.}

Mouse body weights were lower in the HLS compared with both the PF and BL group after three days of unloading (fig $1 \mathrm{~A}$ ). Muscle weights of all assessed hind limb muscles were lower in the HLS group, while only the $m$. plantaris weight was significantly lower in the PF group compared with BL (fig 1 B).

Human subjects did not lose body weight during 7 days of muscle unloading. However, they lost muscle strength (as measured by 1-RM), and muscle mass (as measured by CT-derived thigh muscle CSA and quadriceps CSA) (table 2).

Table 2: Immobilization-induced body composition changes in men.

\begin{tabular}{|l|l|}
\hline $\begin{array}{l}\text { Characteristic } \\
(\mathrm{n}=13)\end{array}$ & $22[18-28]$ \\
\hline Age (years) & $1.78[1.56-1.89]$ \\
\hline Height $(\mathrm{m})$ & $74[55.8-83.1]$ \\
\hline Body Weight $(\mathrm{kg})$ & $23[19.3-25.2]$ \\
\hline BMl $\left(\mathrm{kg} \mathrm{m}^{-2}\right)$ & $-0.24[-2.20-2.07]$ \\
\hline$\Delta$ Body Weight (\%) & $-12.22[-25.37-8.11]^{* *}$ \\
\hline$\Delta 1$-RM (\%) & $-3.43[-6.54-0.98]^{* *}$ \\
\hline$\Delta$ Thigh Muscle CSA (\%) & $-4.86[-8.48--1.04]^{* *}$ \\
\hline$\Delta$ Quadriceps CSA (\%) & \\
\hline
\end{tabular}

Abbreviations: BMI, body mass index; 1-RM, 1 repetition max; CSA, cross-sectional area. Significant differences ${ }^{* *} p<0.01$ are depicted.

\section{Mitochondrial quantity is unaltered during muscle unloading}

It is well-established that there is a decrease in muscle mitochondrial content upon extended hind limb unloading, which subsequently recovers during hind limb reloading (3). To investigate whether the loss of mitochondrial content is already initiated in an early stage of unloading, we quantified several markers of mitochondrial content. None of the mitochondrial proteins were significantly lower in HLS compared with PF or BL (fig $2 \mathrm{~A}$ ). To obtain a more functional overview of the skeletal muscle mitochondrial capacity, citrate synthase and HADH enzyme activity was determined in the murine study, as well as the more glycolytic phosphofructokinase. In agreement with the mitochondrial protein expression data, no differences were found for any of these enzymes (fig $2 \mathrm{C}$ ). In line with the murine data, no significant differences were found for markers of mitochondrial quantity during unloading of human skeletal muscles either (fig 2 B). 


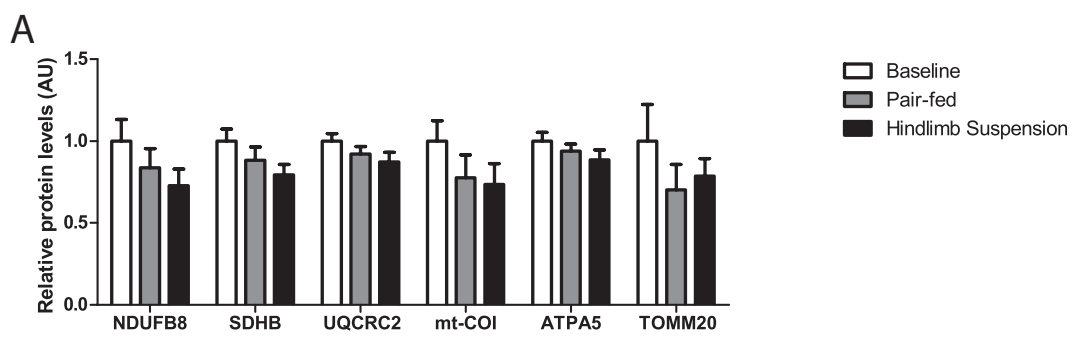

B

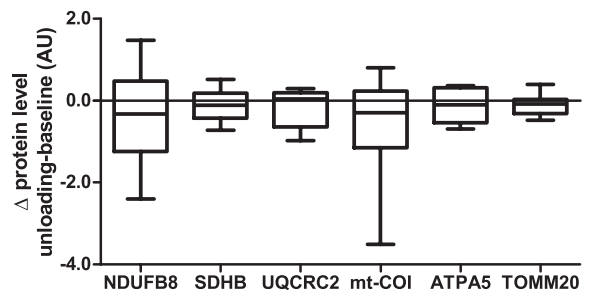

C

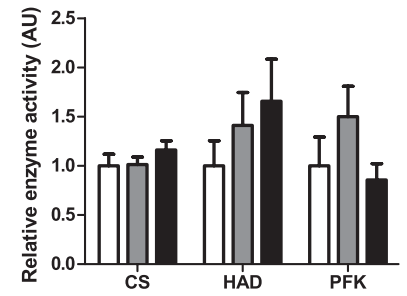

Figure 2: Mitochondrial quantity in response to muscle unloading. Murine $m$. gastrocnemius mitochondrial content-associated protein levels (A), and enzyme activity (C) are depicted. White bars represent $\mathrm{BL}$, grey bars represent PF, black bars represent HLS. Human v. lateralis $\Delta$ (unloadingbaseline) values of mitochondrial content-associated protein levels are depicted (B). No statistical significance was found between indicated bars $(\mathbf{A}, \mathbf{C})$ or between $\triangle \mathrm{UL}-\mathrm{BL}(\mathbf{B})$.

Modulations in receptor-mediated mitophagy- and autophagy-signalling during muscle unloading

HLS had lower BNIP3L and higher BNIP3L-II protein levels compared with BL. In addition, BNIP3 protein levels were higher in HLS. Moreover, both Bnip3l and Bnip3 gene-expression was higher in HLS compared with both BL and PF. FUNDC1 protein levels were lower in HLS compared with BL, while Fundc1 gene-expression was higher in PF compared with both BL and HLS (fig $3 \mathrm{~A}, \mathrm{C}$ ). Pink1 mRNA expression was not different in HLS compared with BL. Interestingly, Parkin protein levels were lower in HLS, without corresponding changes in Park2 mRNA expression (fig $3 \mathrm{~A}, \mathrm{C}$ ). No differences were found in either LC3B-I, LC3B-II or their ratio in the unloaded mice. GABARAPL1 protein levels were higher in HLS compared with PF but not BL. On transcript level however, $L c 3 b$ but not Gabarap/1 expression was potently higher in HLS compared with both PF and BL. Interestingly, autophagy adaptor protein SQSTM1 was higher both on protein and mRNA expression level in HLS. In addition, mRNA expression of Optn was higher in $\mathrm{HLS}$ as well (fig $3 \mathrm{E}, \mathrm{G}$ ).

In the human subjects, both BNIP3L protein levels and BNIP3L mRNA expression increased upon muscle unloading, similar to the BNIP3L-II protein and Bnip3I mRNA expression in the murine study. Moreover, although $\triangle$ protein as well as 


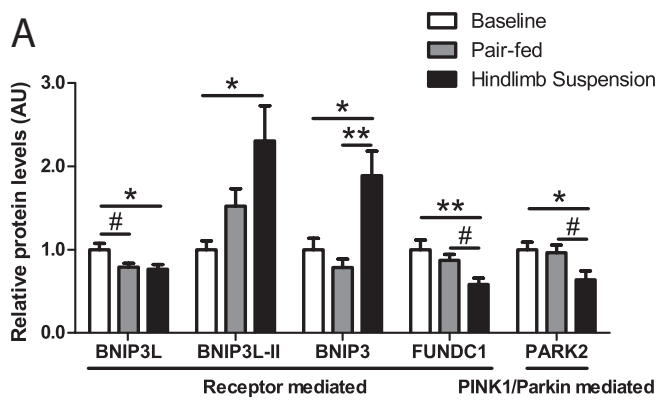

B

C

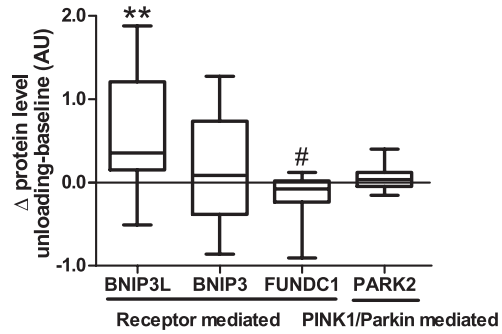

$\mathrm{D}$
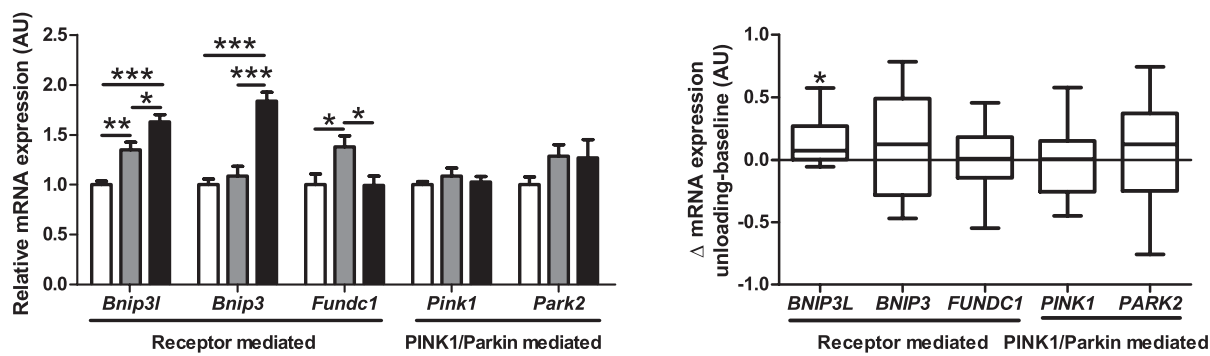

$\mathrm{E}$

$\mathrm{F}$
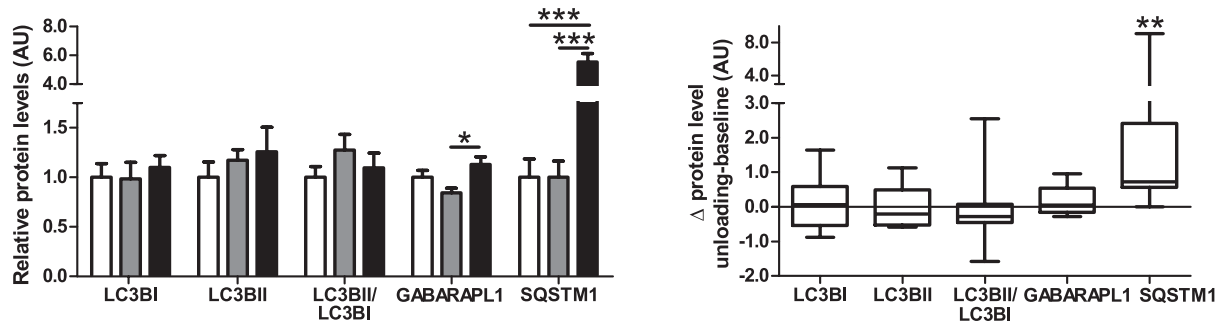

G

$\mathrm{H}$
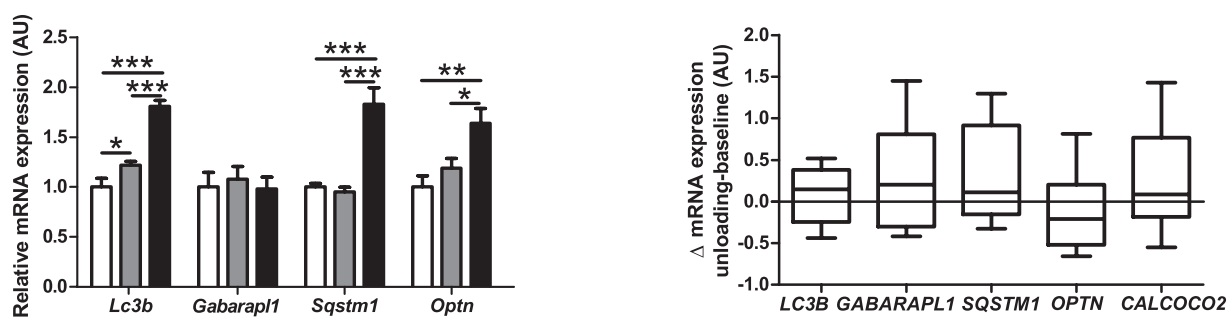

Figure 3: Mitophagy- and autophagy-associated protein and mRNA expression in response to muscle unloading. Murine $m$. gastrocnemius mitophagy-associated protein levels (A) and mRNA expression (C), and autophagy-associated protein levels (E) and mRNA expression (G) are depicted. White bars represent $\mathrm{BL}$, grey bars represent PF, black bars represent HLS. Human $v$. lateralis $\Delta$ (unloading-baseline) values of mitophagy-associated protein levels (B) and mRNA expression (D), and autophagy-associated protein levels (F) and mRNA expression (H) are depicted. Statistical significance is indicated between indicated bars $(\mathbf{A}, \mathbf{C}, \mathbf{E}, \mathbf{G})$ or between $\triangle \mathrm{UL}-\mathrm{BL}(\mathrm{B}, \mathrm{D}, \mathrm{F}, \mathrm{H}){ }^{*} p<0.1,{ }^{*} p<0.05,{ }^{* *} p<0.01,{ }^{* * *} p<0.001$. 
$\triangle$ mRNA expression of BNIP3 and FUNDC1 showed similar patterns compared with the murine study, these data did not reach statistical significance. In addition, no changes for either PINK1 or Parkin on protein or mRNA expression level were found in the human subjects upon muscle unloading (fig $3 \mathrm{~B}, \mathrm{D}$ ). With regard to general autophagy markers, SQSTM1 protein levels were potently increased during muscle unloading in the human subjects, which is in line with the data from the murine study. In addition, no significant changes were found for the autophagy-related mRNA expression in the human subjects (fig $3 \mathrm{~F}, \mathrm{H}$ ).

\section{Transcriptional regulation of mitochondrial biogenesis is decreased during muscle unloading}

Since mitochondrial breakdown and synthesis together determine the mitochondrial content, we also investigated whether skeletal muscle unloading resulted in alterations of mitochondrial biogenesis and the molecular regulation thereof. PPARGC1A, TFAM, and NRF1 protein levels were not different in HLS compared with PF or BL, although TFAM tended to be higher in HLS compared with PF (fig 4 A). In contrast, Ppargc1a and Ppargc1b mRNA expression was found to be lower HLS, without corresponding differences in Ppard, Tfam and Nrf1. Surprisingly, Tfam and Nrf1 mRNA expression were higher in PF compared with $\mathrm{BL}$ (fig $4 \mathrm{C}$ ). In concert with the murine data, no differences in mitochondrial biogenesis related protein or mRNA levels were found during muscle unloading in the human subjects, although PARGC1A mRNA expression tended to be decreased (fig 4 B, D).

\section{Mitochondrial dynamics related signalling is unaltered by muscle unloading}

Since mitochondrial fission is often described as a pre-requisite for mitophagy, we investigated markers for both mitochondrial fission and fusion events. In the murine study, no differences were observed in mitochondrial dynamicsassociated protein levels or mRNA expression (fig 5 A, C). In concert with the murine data, no changes were found for any of the measured mitochondrial dynamics-associated markers in the human subjects (fig 5 B, D). 

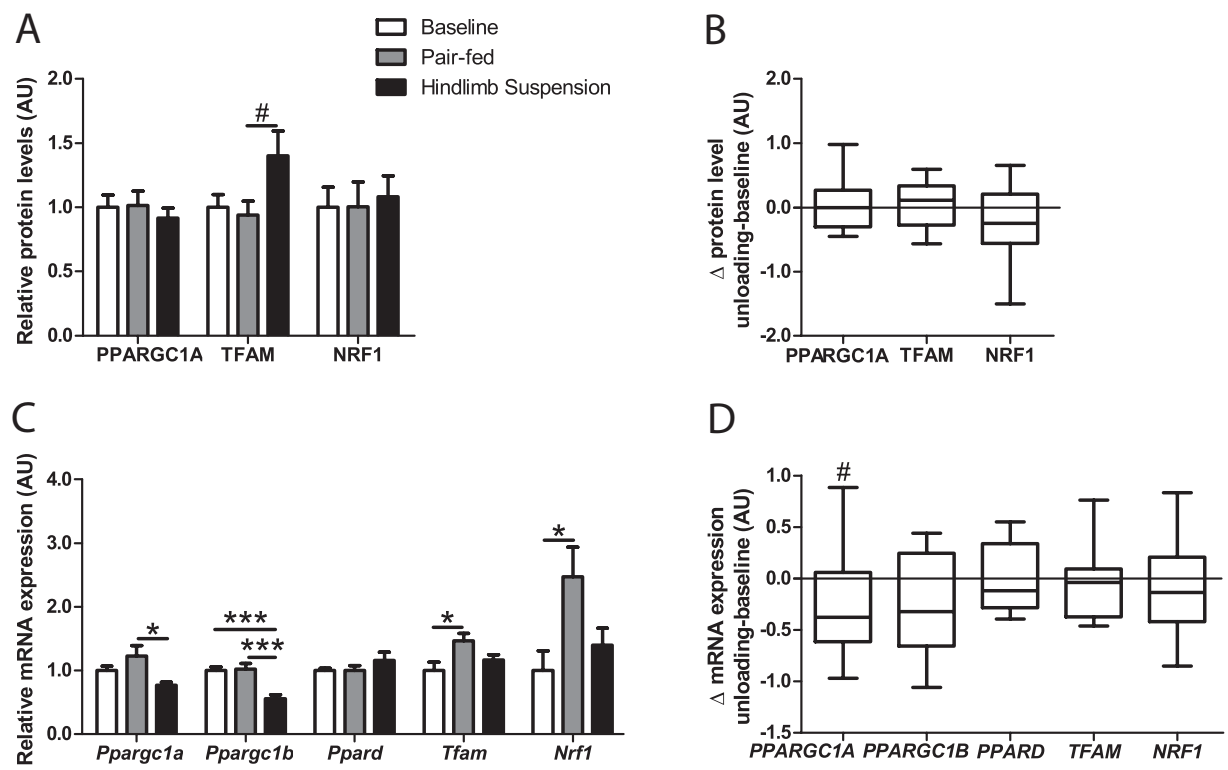

Figure 4: Mitochondrial biogenesis-associated protein and mRNA expression in response to muscle unloading. Murine $m$. gastrocnemius mitochondrial biogenesis-associated protein levels (A) and mRNA expression (C) are depicted. White bars represent BL, grey bars represent PF, black bars represent HLS. Human $v$. lateralis $\Delta$ (unloading-baseline) values mitochondrial biogenesis-associated protein levels (B) and mRNA expression (D) are depicted. Statistical significance is indicated between indicated bars (A, C) or between $\Delta \mathrm{UL}-\mathrm{BL}(\mathbf{B}, \mathbf{D}){ }^{*} p<0.1,{ }^{*} p<0.05$, ${ }_{* *}^{*} p<0.01, * * * p<0.001$.

A
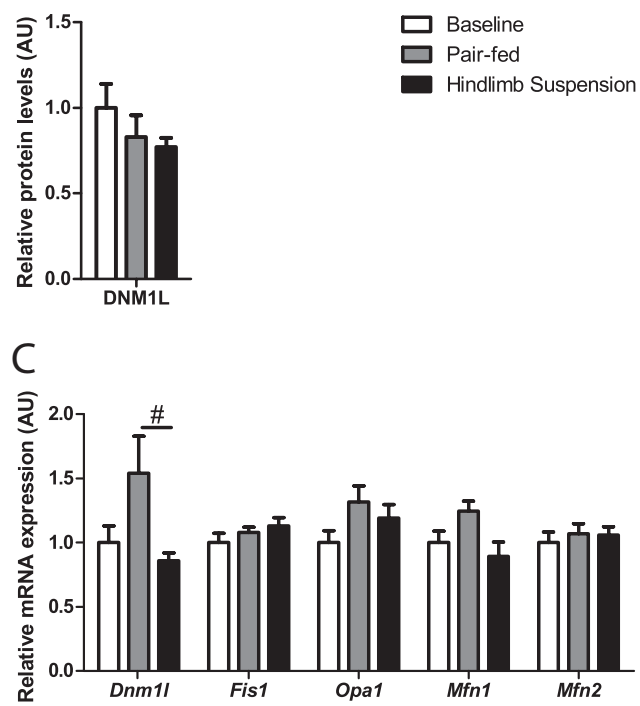

B

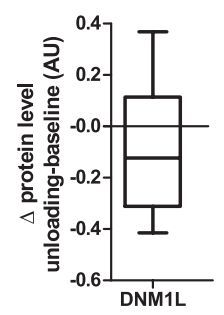

D

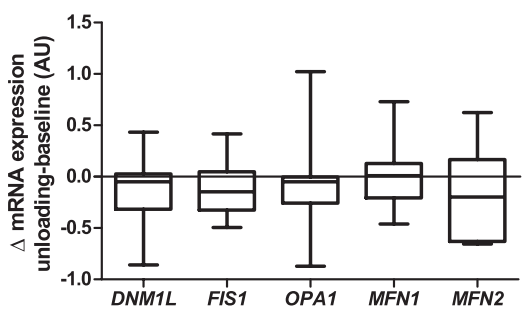

Figure 5: Mitochondrial dynamics-associated protein and mRNA expression in response to muscle unloading. Murine $m$. gastrocnemius mitochondrial dynamics-associated protein levels (A) and mRNA expression (C) are depicted. White bars represent $\mathrm{BL}$, grey bars represent PF, black bars represent HLS. Human $v$. lateralis $\Delta$ (unloading-baseline) values of mitochondrial dynamics-associated protein levels (B) and mRNA expression (D) are depicted. Statistical significance is indicated between indicated bars $(\mathbf{A}, \mathbf{C})$ or between $\triangle \mathrm{UL}-\mathrm{BL}(\mathbf{B}, \mathbf{D}){ }^{\#} p<0.1$. 


\section{Discussion}

The current study shows for the first time that skeletal muscle unloading results in a pattern of early-onset pro-mitophagy signalling in murine skeletal muscle confirmed by a similar signalling pattern in unloaded human skeletal muscle, both still without an apparent decline of skeletal muscle mitochondrial content. These data suggest that the pro-mitophagy signalling precedes the loss of mitochondrial content and therefore might play an important role in the onset of disuse-induced loss of mitochondrial content.

The loss of mitochondrial content and oxidative capacity has been wellestablished in different immobilization models in both rat and murine skeletal muscle (3-10). In line with this, 7 days of strict bedrest, and 14 but not 2 days of human limb immobilization resulted in decreased mitochondrial content markers $(1,2,23)$. Currently, we report no changes in mitochondrial quantity markers in both the murine ( 3 days of HLS) and the human ( 7 days of UL) unloaded muscle, which suggest that the above mentioned changes in molecular signalling are prior to the well-established loss of mitochondrial quantity during muscle unloading.

Mitophagy can be initiated by several mitochondrial-localized mitophagy receptors (e.g. BNIP3, BNIP3L, and FUNDC1), which stimulates their binding to proteins of the LC3/GABARAP family (32-34). Subsequently, the mitochondrion is engulfed in an autophagosomal membrane and broken down via the lysosomal pathway. Previous studies provide mixed evidence, having found lower m. tibialis protein levels of BNIP3 (9), and lower gene-expression but higher protein levels of BNIP3L (10) after 7 days of hind limb immobilization by wrapping or denervation. Moreover, m. gastrocnemius Bnip3 and Bnip3I gene-expression was unchanged after prolonged unloading (4). We report higher transcript and protein abundance of BNIP3 and BNIP3L during the first stage of unloading in mice, indicating more pronounced mitophagy during the initial stages of unloading. Interestingly, the same pattern was found in human unloaded skeletal muscle, suggesting this molecular response is conserved between species.

FUNDC1-mediated mitophagy, activated by post-translational modifications (e.g. by phosphorylation) of available FUNDC1 protein rather than increasing FUNDC1 protein abundance (35-38), remains poorly described during muscle unloading. In our study we show that FUNDC1 protein levels are lower in skeletal muscle unloaded mice, while its mRNA transcript levels are unaltered, suggesting post-translational regulation of FUNDC protein abundance during the 
early phases of unloading. Interestingly, we report the same pattern in human unloaded skeletal muscle. Although it is likely that the observed FUNDC1 depletion contributes to subsequent mitochondrial quantity loss, the causality of this relationship remains to be established.

In a different pathway of mitophagy initiation, the PINK1/Parkin-mediated mitophagy, a loss of mitochondrial membrane potential promotes the stabilization of PINK1, resulting in either the recruitment and activation of the E3 ubiquitin ligase Parkin and downstream autophagy-receptor SQSTM1, or in the Parkin independent recruitment and activation of autophagy-receptors like OPTN and CALCOCO2 (39-41). Like the mitophagy-receptors, these autophagy receptors are able to bind LC3 or GABARAP family proteins. In extension to our data which shows no differences or changes for PINK1 protein or mRNA expression in both the murine and human model, PINK1 protein levels were found unaltered after 7 and 14 days of hind limb immobilization, but were found to be lower after 21 days of immobilization in murine $m$. tibialis anterior (9). The same study reports higher Parkin protein levels after 7 days of hind limb immobilization, while we found lower levels after three days (9). Since these studies show differences in timing, type of muscle analysed, and protocol of muscle unloading, the exact cause of this difference remains to be established. We did not find any differences in PINK1 or Parkin protein or transcript expression in human skeletal muscle after unloading, suggesting that this pathway does not play a major role in the loss of mitochondrial quantity during acute unloading.

Autophagy-related proteins are instrumental for the process of mitophagy, and their transcript levels were generally found to be higher in the unloaded mice compared with the controls in our murine model. Although these differences were only marginally reflected by their protein levels, SQSTM1 protein levels were increased in both the unloaded murine and human skeletal muscle in our study, suggesting similar molecular autophagy-signalling response between species. Combined with data from previous studies, generally showing increased presence of protein and/or transcript abundance of autophagy-related proteins $(4,9,10)$, autophagy-related signalling is likely induced shortly after the start of muscle unloading.

Many rodent studies have been performed to study mitochondrial biogenesis at various time-points and in various different muscles during muscle unloading $(3-8,17,18)$. In short, 1 day of hind limb unloading resulted in decreased expression of many genes involved in mitochondrial biogenesis, except for increased PPARD transcript and protein levels in $m$. gastrocnemius, and 3 days of hind limb unloading resulted in lower PPARGC1A protein and transcript 
abundance in murine $m$. soleus but not in $m$. gastrocnemius $(8,17,18)$. These studies are in concert with our data, showing profoundly lower Ppargc1b mRNA expression in the unloaded muscle, while Ppargc1a mRNA expression was only marginally affected. In addition, it was previously reported that transcript levels of multiple mitochondrial biogenesis and metabolism markers were lower at 4-11 days post human limb immobilization, while another study reported only marginal changes at 2 days $(24,42)$. Our results combined with literature suggest an early-onset decreased transcriptional regulation of mitochondrial biogenesis during muscle unloading.

Skeletal muscle mitochondria are part of a highly dynamic network of organelles, constantly undergoing fission (i.e. separation of mitochondria, regulated by e.g. DNM1L and FIS1) and fusion (i.e. merging of mitochondria, regulated by e.g. OPA1 and MFN1/2) events (15). Since the autophagosomal size is limited, mitochondrial fission is often considered a prerequisite for mitophagy as it isolates small mitochondria from the network $(15,16)$. Although transcript and protein expression data related to mitochondrial fission and fusion is difficult to interpret, partly due to the post-transcriptional nature of their regulation, we included some measurements to obtain a general overview of major regulatory changes. In addition to our results, which show no differences in any of the fission and fusion markers for both the murine and human skeletal muscle unloading models, many other rodent studies have been performed which generally report decreased or equal transcript and protein levels of the above mentioned markers $(3,4,6-9,18)$. Together with the literature, our data make a profound shift from a fission/fusion homeostasis towards dominion of either fission or fusion unlikely in our models.

Although no actual mitophagy or autophagy flux was measured in our samples, we report a comprehensive set of markers involved in the execution and regulation of mitophagy, autophagy, mitochondrial biogenesis, and mitochondrial dynamics measured in the early phase after muscle unloading. Therefore, the increased protein abundance of the mitophagy and autophagy-related proteins might also be the result of impaired breakdown instead of increased synthesis. Moreover, we describe associations between changes in the processes of autophagy/mitophagy and mitochondrial biogenesis, but the causality of these associations remains unclear in our study. However, the current results provide novel insights which may have implications for hospitalized and bed-bound patients, and to inactive patients with chronic diseases as well. 
In conclusion, we now show that both in mice and men skeletal muscle unloading results in early-onset molecular marker patterns indicative of increased receptor-mediated mitophagy and decreased mitochondrial biogenesis, before the loss of mitochondrial content. These results emphasize the early onset of skeletal muscle disuse-induced mitochondrial remodelling, which is likely to underlie the loss of mitochondrial quantity in a later stage of muscle disuse. Moreover, we report similar expression patterns in the murine and human skeletal muscle, which suggests this molecular response is conserved between species and emphasize the early-onset of human skeletal muscle disuse-induced mitochondrial remodelling.

\section{Funding}

The original work from which human muscle biopsies were obtained (27) was funded by TI Food and Nutrition, a public-private partnership on precompetitive research in food and nutrition. The mouse study was funded by a grant from the Lung Foundation/Netherlands Asthma Foundation (NAF 3.2.07.017) and the transnational University Limburg (tUL). 


\section{References}

1. Gram M, Vigelso A, Yokota T, Hansen CN, Helge JW, Hey-Mogensen M, et al. Two weeks of one-leg immobilization decreases skeletal muscle respiratory capacity equally in young and elderly men. Experimental gerontology. 2014 Oct;58:269-78. PubMed PMID: 25193555.

2. Dirks ML, Wall BT, van de Valk B, Holloway TM, Holloway GP, Chabowski A, et al. One Week of Bed Rest Leads to Substantial Muscle Atrophy and Induces Whole-Body Insulin Resistance in the Absence of Skeletal Muscle Lipid Accumulation. Diabetes. 2016 Oct;65(10):2862-75. PubMed PMID: 27358494.

3. Remels $A H$, Pansters NA, Gosker HR, Schols AM, Langen RC. Activation of alternative NF-kappaB signaling during recovery of disuse-induced loss of muscle oxidative phenotype. American journal of physiology Endocrinology and metabolism. 2014 Mar;306(6):E615-26. PubMed PMID: 24425759.

4. $\quad$ Liu J, Peng Y, Cui Z, Wu Z, Qian A, Shang P, et al. Depressed mitochondrial biogenesis and dynamic remodeling in mouse tibialis anterior and gastrocnemius induced by 4-week hindlimb unloading. IUBMB life. 2012 Nov;64(11):901-10. PubMed PMID: 23024034.

5. Cassano P, Sciancalepore AG, Pesce V, Fluck M, Hoppeler H, Calvani M, et al. Acetyl-L-carnitine feeding to unloaded rats triggers in soleus muscle the coordinated expression of genes involved in mitochondrial biogenesis. Biochimica et biophysica acta. 2006 Sep-Oct;1757(9-10):1421-8. PubMed PMID: 16814248.

6. Qi Z, Zhang Y, Guo W, Ji L, Ding S. Increased insulin sensitivity and distorted mitochondrial adaptations during muscle unloading. International journal of molecular sciences. 2012 Dec 11;13(12):16971-85. PubMed PMID: 23443131. Pubmed Central PMCID: 3546734.

7. Wagatsuma A, Kotake N, Kawachi T, Shiozuka M, Yamada S, Matsuda R. Mitochondrial adaptations in skeletal muscle to hindlimb unloading. Molecular and cellular biochemistry. 2011 Apr;350(1-2):1-11. PubMed PMID: 21165677.

8. Cannavino J, Brocca L, Sandri M, Bottinelli R, Pellegrino MA. PGC1-alpha overexpression prevents metabolic alterations and soleus muscle atrophy in hindlimb unloaded mice. The Journal of physiology. 2014 Oct 15;592(20):4575-89. PubMed PMID: 25128574. Pubmed Central PMCID: 4287741.

9. Kang C, Yeo D, Ji LL. Muscle Immobilization Activates Mitophagy and Disrupts Mitochondrial Dynamics in Mice. Acta physiologica. 2016 Apr 16. PubMed PMID: 27083499.

10. Vainshtein A, Desjardins EM, Armani A, Sandri M, Hood DA. PGC-1alpha modulates denervation-induced mitophagy in skeletal muscle. Skeletal muscle. 2015;5:9. PubMed PMID: 25834726. Pubmed Central PMCID: 4381453.

11. Palikaras K, Tavernarakis N. Mitochondrial homeostasis: The interplay between mitophagy and mitochondrial biogenesis. Experimental gerontology. 2014 Jan 28(56):182-8. PubMed PMID: 24486129.

12. Correia JC, Ferreira DM, Ruas JL. Intercellular: local and systemic actions of skeletal muscle PGC-1s. Trends in endocrinology and metabolism: TEM. 2015 Jun;26(6): 305-14. PubMed PMID: 25934582. 
13. Zhang T, Xue L, Li L, Tang C, Wan Z, Wang R, et al. BNIP3 Protein Suppresses PINK1 Kinase Proteolytic Cleavage to Promote Mitophagy. The Journal of biological chemistry. 2016 Oct 07;291(41):21616-29. PubMed PMID: 27528605. Pubmed Central PMCID: 5076832.

14. Gao F, Chen D, Si J, Hu Q, Qin Z, Fang M, et al. The mitochondrial protein BNIP3L is the substrate of PARK2 and mediates mitophagy in PINK1/PARK2 pathway. Human molecular genetics. 2015 May 01;24(9):2528-38. PubMed PMID: 25612572.

15. Romanello V, Sandri M. Mitochondrial Quality Control and Muscle Mass Maintenance. Frontiers in physiology. 2015;6:422. PubMed PMID: 26793123. Pubmed Central PMCID: 4709858.

16. Gomes LC, Scorrano L. Mitochondrial morphology in mitophagy and macroautophagy. Biochimica et biophysica acta. 2013 Jan;1833(1):205-12. PubMed PMID: 22406072.

17. Mazzatti DJ, Smith MA, Oita RC, Lim FL, White AJ, Reid MB. Muscle unloadinginduced metabolic remodeling is associated with acute alterations in PPARdelta and UCP-3 expression. Physiological genomics. $2008 \mathrm{Jul}$ 15;34(2):149-61. PubMed PMID: 18445701.

18. Cannavino J, Brocca L, Sandri M, Grassi B, Bottinelli R, Pellegrino MA. The role of alterations in mitochondrial dynamics and PGC-1alpha over-expression in fast muscle atrophy following hindlimb unloading. The Journal of physiology. 2015 Apr 15; 593(8):1981-95. PubMed PMID: 25565653. Pubmed Central PMCID: 4405755.

19. Furuya N, Ikeda S, Sato S, Soma S, Ezaki J, Oliva Trejo JA, et al. PARK2/Parkinmediated mitochondrial clearance contributes to proteasome activation during slow-twitch muscle atrophy via NFE2L1 nuclear translocation. Autophagy. 2014 Apr; 10(4):631-41. PubMed PMID: 24451648. Pubmed Central PMCID: 4091150.

20. Cobley JN, Bartlett JD, Kayani A, Murray SW, Louhelainen J, Donovan T, et al. PGC-1alpha transcriptional response and mitochondrial adaptation to acute exercise is maintained in skeletal muscle of sedentary elderly males. Biogerontology. 2012 Dec;13(6):621-31. PubMed PMID: 23187721.

21. Safdar A, Hamadeh MJ, Kaczor JJ, Raha S, Debeer J, Tarnopolsky MA. Aberrant mitochondrial homeostasis in the skeletal muscle of sedentary older adults. PloS one. 2010 May 24;5(5):e10778. PubMed PMID: 20520725. Pubmed Central PMCID: 2875392.

22. Drummond MJ, Addison O, Brunker L, Hopkins PN, McClain DA, LaStayo PC, et al. Downregulation of E3 ubiquitin ligases and mitophagy-related genes in skeletal muscle of physically inactive, frail older women: a cross-sectional comparison. The journals of gerontology Series A, Biological sciences and medical sciences. 2014 Aug; 69(8):1040-8. PubMed PMID: 24526667. Pubmed Central PMCID: 4111292.

23. Abadi A, Glover El, Isfort RJ, Raha S, Safdar A, Yasuda N, et al. Limb immobilization induces a coordinate down-regulation of mitochondrial and other metabolic pathways in men and women. PloS one. 2009 Aug 05;4(8):e6518. PubMed PMID: 19654872. Pubmed Central PMCID: 2716517.

24. Reich KA, Chen YW, Thompson PD, Hoffman EP, Clarkson PM. Forty-eight hours of unloading and $24 \mathrm{~h}$ of reloading lead to changes in global gene expression patterns related to ubiquitination and oxidative stress in humans. J Appl Physiol (1985). 2010 Nov;109(5):1404-15. PubMed PMID: 20798274. Pubmed Central PMCID: 3774122. 
25. Standley RA, Distefano G, Pereira SL, Tian M, Kelly OJ, Coen PM, et al. Effects of beta-hydroxy-beta-methylbutyrate on skeletal muscle mitochondrial content and dynamics, and lipids after 10 days of bed rest in older adults. J Appl Physiol (1985). 2017 Nov 1;123(5):1092-100. PubMed PMID: 28705993.

26. Grundy D. Principles and standards for reporting animal experiments in The Journal of Physiology and Experimental Physiology. The Journal of physiology. 2015 Jun 15; 593(12):2547-9. PubMed PMID: 26095019. Pubmed Central PMCID: 4500341.

27. Backx EMP, Hangelbroek R, Snijders T, Verscheijden ML, Verdijk LB, de Groot L, et al. Creatine Loading Does Not Preserve Muscle Mass or Strength During Leg Immobilization in Healthy, Young Males: A Randomized Controlled Trial. Sports medicine. 2017 Aug;47(8):1661-71. PubMed PMID: 28054322. Pubmed Central PMCID: 5507980.

28. Pansters NA, Schols AM, Verhees KJ, de Theije CC, Snepvangers FJ, Kelders MC, et al. Muscle-specific GSK-3beta ablation accelerates regeneration of disuseatrophied skeletal muscle. Biochimica et biophysica acta. 2015 Mar;1852(3): 490-506. PubMed PMID: 25496993.

29. Langen RC, Van Der Velden JL, Schols AM, Kelders MC, Wouters EF, Janssen-Heininger YM. Tumor necrosis factor-alpha inhibits myogenic differentiation through MyoD protein destabilization. FASEB journal : official publication of the Federation of American Societies for Experimental Biology. 2004 Feb;18(2):227-37. PubMed PMID: 14769817.

30. Gouspillou G, Sgarioto N, Norris B, Barbat-Artigas S, Aubertin-Leheudre M, Morais $J A$, et al. The relationship between muscle fiber type-specific PGC-1alpha content and mitochondrial content varies between rodent models and humans. PloS one. 2014;9(8):e103044. PubMed PMID: 25121500. Pubmed Central PMCID: 4133187.

31. Slot IG, Schols AM, de Theije CC, Snepvangers FJ, Gosker HR. Alterations in Skeletal Muscle Oxidative Phenotype in Mice Exposed to 3 Weeks of Normobaric Hypoxia. Journal of cellular physiology. 2016 Feb;231(2):377-92. PubMed PMID: 26129845.

32. Yamaguchi O, Murakawa T, Nishida K, Otsu K. Receptor-mediated mitophagy. Journal of molecular and cellular cardiology. 2016 Mar 25(95):50-6. PubMed PMID: 27021519.

33. Wei H, Liu L, Chen Q. Selective removal of mitochondria via mitophagy: distinct pathways for different mitochondrial stresses. Biochimica et biophysica acta. 2015 Oct;1853(10 Pt B):2784-90. PubMed PMID: 25840011.

34. Stotland A, Gottlieb RA. Mitochondrial quality control: Easy come, easy go. Biochimica et biophysica acta. 2015 Oct;1853(10 Pt B):2802-11. PubMed PMID: 25596427. Pubmed Central PMCID: 4501896.

35. Chen M, Chen Z, Wang Y, Tan Z, Zhu C, Li Y, et al. Mitophagy receptor FUNDC1 regulates mitochondrial dynamics and mitophagy. Autophagy. 2016 Apr 2;12(4):689-702. PubMed PMID: 27050458. Pubmed Central PMCID: 4836026.

36. Wu W, Tian W, Hu Z, Chen G, Huang L, Li W, et al. ULK1 translocates to mitochondria and phosphorylates FUNDC1 to regulate mitophagy. EMBO reports. 2014 May;15(5):566-75. PubMed PMID: 24671035. Pubmed Central PMCID: 4210082.

37. Liu L, Feng D, Chen $G$, Chen $M$, Zheng $Q$, Song $P$, et al. Mitochondrial outermembrane protein FUNDC1 mediates hypoxia-induced mitophagy in mammalian cells. Nature cell biology. 2012 Feb;14(2):177-85. PubMed PMID: 22267086. 
38. Lv M, Wang C, Li F, Peng J, Wen B, Gong $Q$, et al. Structural insights into the recognition of phosphorylated FUNDC1 by LC3Bin mitophagy. Protein \& cell. 2017 Jan; 8(1):25-38. PubMed PMID: 27757847. Pubmed Central PMCID: 5233613.

39. Matsuda N. Phospho-ubiquitin: upending the PINK-Parkin-ubiquitin cascade. Journal of biochemistry. 2016 Apr;159(4):379-85. PubMed PMID: 26839319.

40. Lazarou M, Sliter DA, Kane LA, SarrafSA, Wang C, Burman JL, et al. The ubiquitin kinase PINK1 recruits autophagy receptors to induce mitophagy. Nature. 2015 Aug 20; 524(7565):309-14. PubMed PMID: 26266977.

41. Eiyama A, Okamoto K. PINK1/Parkin-mediated mitophagy in mammalian cells. Current opinion in cell biology. 2015 Apr;33:95-101. PubMed PMID: 25697963.

42. Chen YW, Gregory CM, Scarborough MT, Shi R, Walter GA, Vandenborne K. Transcriptional pathways associated with skeletal muscle disuse atrophy in humans. Physiological genomics. 2007 Nov 14;31(3):510-20. PubMed PMID: 17804603. 


\section{6}

Coordinated regulation of skeletal muscle mass and metabolic plasticity during recovery from disuse

A.E.M Kneppers, P.A. Leermakers, N.A.M. Pansters, E.M.P. Backx, H.R. Gosker, L.J.C. van Loon, A.M.W.J. Schols, R.C.J. Langen, L.B. Verdijk

FASEB J. 2018 Aug 22:fj201701403RRR. 


\section{Abstract}

Skeletal muscle regeneration after disuse is essential for muscle maintenance and involves the regulation of both mass- and metabolic plasticity-related processes. However, the relation between these processes during recovery from disuse remains unclear. In this study, we explored the potential interrelationship between the molecular regulation of muscle mass and oxidative metabolism during recovery from disuse. Molecular profiles were measured in biopsies from the vastus lateralis of healthy men after 1-leg cast immobilization and after 1 wk reloading, and in mouse gastrocnemius obtained before and after hindlimb suspension and during reloading ( $\mathrm{RL}-1,-2,-3,-5$, and $-8 \mathrm{~d}$ ). Cluster analysis of the human recovery response revealed correlations between myogenesis and autophagy markers in 2 clusters, which were distinguished by the presence of markers of early myogenesis, autophagosome formation, and mitochondrial turnover vs. markers of late myogenesis, autophagy initiation, and mitochondrial mass. In line with these findings, an early transient increase in B-cell lymphoma-2 interacting protein 3 (BNIP3) and sequestosome-1 (SQSTM1) protein, and GABA type A receptor-associated protein like-1 (GABARAPL1) protein and $m R N A$ and a late increase in myomaker (MYMK) and myosin heavy chain-8 (MYH8) mRNA, microtubule-associated protein 1 light chain 3 (MAP1LC3)II:I ratio, and FUN14 domain-containing-1 (FUNDC1) mRNA and protein were observed in mice. In summary, the regulatory profiles of protein, mitochondrial, and myonuclear turnover are correlated and temporally associated, suggesting a coordinated regulation of muscle mass- and oxidative metabolism-related processes during recovery from disuse. 


\section{Introduction}

Skeletal muscles are essential to generate the forces for postural support and physical function. Furthermore, representing half of the body mass and being highly metabolically active, skeletal muscle tissue is an important site for control of metabolism (1). These functions are supported by the muscles' biochemical and morphologic makeup, which are tightly regulated and can rapidly adapt to alterations in mechanical and metabolic demands - referred to as skeletal muscle plasticity.

Muscle disuse results from low skeletal muscle mechanical loading, such as occurs with bed rest during hospitalization after surgery or acute illness, with immobilization after a fracture, and with certain chronic diseases. It is well established that disuse leads to a reduction of skeletal muscle strength (2) and mass $(2,3)$, which results from a negative balance in muscle protein turnover $(2,4)$. In addition, disuse affects muscle quality, including a transition from a slow to fast fiber type (5-7), a shift from fat oxidation toward glycolysis (8), a decrease in mitochondrial mass and function (9-12), and a reduction in vascularization (13).

The above-mentioned qualitative alterations in response to disuse collectively reduce the capacity for oxidative metabolism. It has been suggested that these metabolic alterations represent an adaptation to a decreased energy requirement resulting from a reduction in the level of protein turnover upon disuse (8). On the other hand, a study showed that the inhibition of disuseinduced mitochondrial alterations prevents the loss of muscle mass (12). These findings indicate that muscle disuse-induced mass and metabolic alterations are highly interdependent.

Although disuse-induced alterations in skeletal muscle mass and function may be part of a normal physiologic response, the negative implications of muscle disuse atrophy for general health, quality of life (14), and even survival (15-17) have been clearly demonstrated. Both nutritional interventions and exercise mimetics have been used to prevent muscle disuse atrophy, but none serve as a complete substitute for the mechanical and metabolic stimulation during normal muscle use (18-22). Nevertheless, in otherwise healthy individuals, a normalization of muscle mass and function occurs upon reloading after disuse $(23,24)$. However, the negative consequences of disuse may persist when muscle regeneration is impaired, as occurs in elderly (25-27) and chronically ill persons (28). To identify and target factors that hamper skeletal muscle recovery during 
resumed physical activity after disuse in such persons, a better understanding of the molecular alterations driving skeletal muscle plasticity during reloadinginduced recovery is imperative.

In contrast to the wealth of data on the molecular alterations during skeletal muscle disuse in both humans and animals, insight into the molecular response to reloading to induce skeletal muscle regeneration is less abundant and depends mainly on data from studies in rodents. From these studies, it is clear that protein synthesis signaling related to the control of mRNA translation is increased upon reloading after disuse $(27,29,30)$ and that markers of the ubiquitin proteasome system (UPS) return to baseline levels during reloading $(24,30)$. Rather than a passive normalization of the protein turnover balance, several studies suggest a transient activation of protein degradation signaling during reloading-induced recovery, particularly of the autophagy-lysosome pathway $(24,27,31,32)$. In addition, myogenesis seems to be activated during skeletal muscle reloading $(4,30,33)$.

These reloading-induced molecular alterations are also implicated in intrinsic remodeling of skeletal muscle. Indeed, metabolic alterations take place upon skeletal muscle reloading, including an activation of processes that regulate mitochondrial biogenesis and function $(32,34,35)$ and an increase in mitochondrial mass $(32,34)$. Similar to the interdependency of mass and metabolic alterations upon muscle disuse, these processes may be linked during reloading-induced recovery. However, such a potential relation is largely understudied. In the present study, we therefore sought to provide insight in the interrelationship between the regulation of muscle mass and muscle metabolic plasticity during recovery from disuse. A better understanding of these relations is essential for the identification of the molecular origin, and subsequent treatment, of impaired remobilization-induced skeletal muscle recovery in the elderly and chronically ill. We explored these relations by measuring a selected set of indicators and molecular regulators and mediators of muscle mass- and oxidative metabolism-related processes and studied their interrelationship during reloading-induced recovery after disuse in both humans and rodents. 


\section{Materials and Methods}

\section{Subjects, study design, and measurements}

Skeletal muscle molecular profiles of 14 healthy young men (age, $23 \pm 1 \mathrm{yr}$; body mass index, $22.9 \pm 0.6 \mathrm{~kg} / \mathrm{m2}$ ) who participated in a double-blind, randomized, placebo-controlled intervention trial (www.clinicaltrials.gov; NCT01894737) were analyzed. Written informed consent was obtained from all subjects, and the study was approved by the Maastricht University Medical Center+ (Maastricht, The Netherlands) ethics review board (13-3-023) and performed in accordance with the Declaration of Helsinki. The study design and primary data were published previously (36). In brief, participants were subjected to $7 \mathrm{~d}$ of 1-leg immobilization by means of a full leg cast [unloading (UL)], followed by $7 \mathrm{~d}$ of remobilization [reloading (RL)]. Subjects were asked to maintain habitual dietary intake and refrain from any heavy physical exercise during the entire intervention period. Their compliance was checked and confirmed by dietary intake records and activity journals. Furthermore, the subjects consumed standardized meals the evening before all test days. Needle biopsies from the vastus lateralis of the casted leg were taken after an overnight fast after UL and again after RL. Samples were frozen in liquid nitrogen and stored at $-80^{\circ} \mathrm{C}$ until further analysis. Furthermore, muscle mass, measured by cross-sectional area (CSA) was assessed by a computed tomographic scan of the upper legs, muscle strength was assessed with a 1-repetition maximum (1-RM) knee extension test, and body weight was measured with a digital scale. None of the subjects performed progressive resistance-type exercise training or took creatine supplements or any medication that would interfere with muscle metabolism in the 6 mo before the study.

Only subjects with a complete set of available muscle biopsies were included in the current subanalysis $(n=14)$, of whom 5 received placebo and 9 received creatine supplementation. Based on an increase of $<10 \mathrm{mM} / \mathrm{kg}$ in muscle total creatine content (36), 4 subjects were considered nonresponders to creatine loading. Furthermore, creatine supplementation did not have any impact on muscle disuse or subsequent recovery (36).

\section{Animals and study design}

The animal study was approved by the Institutional Animal Care Committee of Maastricht University (DEC-2009-074). The study design has been published $(30,34)$. In the current subanalysis, only the control (i.e., GSK-3ßfl/fl MLC1f-Cre-/-) animals were used. In brief, 13-wk-old male C57/BI6 mice were housed in a temperature-controlled room $\left(21-22{ }^{\circ} \mathrm{C}\right)$ with a $12-12 \mathrm{~h}$ light-dark cycle and 
standard chow pellets and water ad libitum. Mice were subjected to either no experimental procedure (baseline, BL; $n=9$ ), $14 \mathrm{~d}$ of muscle $U \mathrm{~L}$ by hindlimb suspension ( $U L, n=8$ ), or $1,2,3,5$, or $8 d$ of $R L$ after hindlimb suspension ( $n=8$ per time point). Mice were euthanized at each time point by injection with pentobarbital sodium, and hindlimb muscles were excised with standardized dissection methods. Muscles were cleaned of excess fat and connective tissue, pair weighed on an analytical scale, and snap frozen in liquid nitrogen (biochemical analyses) or embedded in Tissue-Tek optimal cutting temperature compound (Sakura Finetek, Zoeterwolde, The Netherlands) and frozen in melting isopentane (histochemical analyses). Samples were stored at $-80^{\circ} \mathrm{C}$ until further analysis. The gastrocnemius was used for protein and mRNA analyses, as its response to hindlimb UL is pronounced and well characterized in studies (37-39). Furthermore, it has a fiber type composition and distribution that is representative of many muscles in the hindlimb and is similar to the fiber type composition observed in the human vastus lateralis.

\section{Protein isolation and Western blot analysis}

For protein analysis, frozen mouse gastrocnemius was powdered and lysed in a whole-cell lysis buffer (50 mM Tris, $150 \mathrm{mM} \mathrm{NaCl}, 10 \%$ glycerol, $0.5 \%$ Nonidet P40, $1 \mathrm{mM}$ EDTA, $500 \mu \mathrm{M}$ Na3VO4, $500 \mu \mathrm{M} \mathrm{NaF}, 100 \mu \mathrm{M} \beta$-glycerophosphate, $100 \mu \mathrm{M}$ Na4P2O7, $1 \mathrm{mM}$ DTT, $10 \mu \mathrm{g} / \mathrm{ml}$ leupeptin, and $1 \%$ aprotinin) for $1 \mathrm{~h}$ on a tube rotator at $4{ }^{\circ} \mathrm{C}$, followed by $30 \mathrm{~min}$ of centrifugation at $14,000 \mathrm{~g}$ at $4{ }^{\circ} \mathrm{C}$. Lysates were aliquoted in sample buffer [0.25M Tris-HCL, 8\% (w/v) SDS, $40 \%(\mathrm{v} / \mathrm{v})$ glycerol, $0.4 \mathrm{M}$ DTT, and $0.02 \%(\mathrm{w} / \mathrm{v})$ bromophenol blue], boiled for $5 \mathrm{~min}$ at $95{ }^{\circ} \mathrm{C}$, and stored at $-80^{\circ} \mathrm{C}$. Human vastus lateralis biopsies were homogenized in lysis buffer ( $20 \mathrm{mM}$ Tris-HCL, 1\% Nonidet P40, $5 \mathrm{mM}$ EDTA, $2 \mathrm{mM}$ Na3VO4, $100 \mathrm{mM} \mathrm{NaF}, 10 \mathrm{mM}$ Na4P2O7, $10 \mu \mathrm{g} / \mathrm{ml}$ leupeptin, $10 \mu \mathrm{g} / \mathrm{ml}$ aprotinin, $3 \mathrm{mM}$ benzamidine, and $1 \mathrm{mM}$ PMSF) using an Ultra-Turrax (IKA Works, Wilmington, NC, USA). Lysates were centrifuged for $10 \mathrm{~min}$ at 10,000 $\mathrm{g}$ at $4{ }^{\circ} \mathrm{C}$. Lysates were aliquoted in sample buffer [0.35M Tris-HCL, $10 \%(\mathrm{w} / \mathrm{v})$ SDS, 30\% (v/v) glycerol, 0.6M DTT, and 0.02\% bromophenol blue], boiled for $5 \mathrm{~min}$ at $100{ }^{\circ} \mathrm{C}$, and stored at $-80{ }^{\circ} \mathrm{C}$. Total protein concentration was determined with the Pierce BCA Protein Assay Kit (Thermo Fisher Scientific, Waltham, MA, USA), according to the manufacturer's instructions.

Ten micrograms protein was separated on a Criterion XT Precast Bis-Tris Gel (Bio-Rad, Hercules, CA, USA) in XT-3-[N-morpholino]propanesulfonic acid or XT-3-[N-morpholino]ethanesulfonic acid running buffer (Bio-Rad) by gel electrophoresis. Proteins were transferred to a $0.45-\mu \mathrm{m}$ nitrocellulose membrane (Bio-Rad) by electroblotting at $100 \mathrm{~V}$ for $60 \mathrm{~min}$ in transfer buffer [25 mM Tris, $192 \mathrm{mM}$ glycine, 20\% (v/v) methanol]. For total protein 
detection, membranes were stained with PonceauS solution $(0.2 \%$ PonceauS in $1 \%$ acetic acid; MilliporeSigma, Burlington, MA, USA) for 3-5 min, washed in double-distilled $\mathrm{H} 2 \mathrm{O}$ for $30 \mathrm{~s}$ on a shaker, and imaged with the Amersham imager 600RGB (GE Healthcar, Chicago, IL, USA). The membranes were blocked for $1 \mathrm{~h}$ at room temperature in Tris-buffered saline with Tween 20 [TBST; 20 $\mathrm{mM}$ Tris, $137 \mathrm{mM} \mathrm{NaCl}, 0.1 \%(\mathrm{v} / \mathrm{v})$ Tween 20, pH 7.6] containing 5\% (w/v) nonfat dry milk (Campina, Eindhoven, The Netherlands). The membranes were washed in TBST, followed by overnight incubation at $4{ }^{\circ} \mathrm{C}$ with primary antibody diluted in TBST with $3 \%$ bovine serum albumin (Table S1). After a wash in TBST, membranes were incubated with a peroxidase-conjugated secondary antibody solution (Vector Laboratories, Burlingame, CA, USA) for $1 \mathrm{~h}$ at room temperature and targets were visualized by chemiluminescence (Supersignal West PICO or FEMTO Chemiluminescent substrate; Thermo Fisher Scientific), according to the manufacturer's instructions, and detected with the Amersham Imager 600RGB. Signals were quantified with Image Quant TL software (GE Healthcare). Samples from each group were distributed within and between blots, to allow for correction of between-session variation (40), and protein expression and phosphorylation levels were corrected for protein loading assessed by Ponceaus.

\section{RNA isolation and RT-qPCR}

For RNA analysis, powdered mouse gastrocnemius was lysed in RLT solution containing 1\% 2-ME. Total RNA was extracted using an on-column RNA isolation kit (Qiagen, Hilden, Germany) according to the manufacturer's instructions and stored at $-80^{\circ} \mathrm{C}$. Human vastus lateralis biopsies were lysed in Trizol (Thermo Fisher Scientific). Total RNA was isolated by phenol-chloroform extraction and subsequent precipitation from the aqueous phase with glycogen-containing isopropanol. RNA was reconstituted in nuclease-free $\mathrm{H}_{2} \mathrm{O}$ and stored at $-80^{\circ} \mathrm{C}$.

RNA concentration was determined with a spectrophotometer (Nanodrop ND-1000 UV-Vis; Thermo Fisher Scientific). RNA (400 ng/sample) was reverse transcribed into CDNA with a Transcriptor CDNA synthesis kit (Roche, Basel, Switzerland), according to the manufacturer's instructions. Primers were designed based on Ensemble transcript sequences and ordered from MilliporeSigma, with primer details shown in Table S2. qPCR reactions contained Sensimix Sybr Rox (GC Biotech, Alphen aan den Rijn, The Netherlands) and primer mix and were run in a 384-well white opaque plate (Roche Diagnostics, Indianapolis, IN, USA) on a LightCycler 480 system (Roche Diagnostics). Melting curves were analyzed to verify specificity of the amplification, and relative quantity of the targets was assessed by LinRegPCR software (v2014.8; http://www.hartfaalcentrum.nl) (Heart Failure Research 
Centre, Amsterdam, The Netherlands). Study-specific reference genes (Table S2) were used to calculate a GeNorm correction factor (https://genorm.cmgg.be/), which was used to normalize expression levels of the target genes.

\section{Immunohistochemistry}

OCT-embedded, frozen mouse gastrocnemius was cut $(7 \mu \mathrm{m})$, defrosted, and air dried at room temperature. Tissue was fixed with $4 \%$ paraformaldehyde and permeabilized with $0.2 \%$ Triton X-100 in PBS. Subsequently, samples were treated for $60 \mathrm{~min}$ with $3 \% \mathrm{H} 2 \mathrm{O} 2$, blocked for $60 \mathrm{~min}$ in blocking solution $[5 \%(\mathrm{v} / \mathrm{v})$ goat serum, $2 \%(\mathrm{w} / \mathrm{v})$ bovine serum albumin, 1:40 mouse-on-mouse blocking reagent (MOM; Vector Laboratories)] at room temperature, and incubated overnight at $4{ }^{\circ} \mathrm{C}$ with primary antibodies directed against laminin and paired box (PAX)-7 (1:250) (Table S1). Samples were incubated in horseradish peroxidase-conjugated streptavidin and goat anti-rabbit 555 (1:200) for 60 min at room temperature, and subsequently for $7 \mathrm{~min}$ in tyramide working solution and reaction stop reagent, according to the manufacturer's protocol (Tyramide SuperBoost Kit; Thermo Fisher Scientific). Nuclei were stained with DAPI, after which cover slips were mounted with Mowiol (MilliporeSigma). After staining, images were digitally captured with fluorescence microscopy (Olympus IX-81; Olympus, Hamburg, Germany) connected to a digital camera (EXi Aqua; Qlmaging, Surrey, BC, Canada) at x400 magnification. Image processing and quantitative analyses were performed with ImageJ (National Institutes of Health, Bethesda, MD, USA).

Laminin staining was used to determine the cell borders. Within each image, the mean fiber CSA, the number of myonuclei per fiber, and the mean fiber CSA per nucleus were quantified. Satellite cells (SCS) were manually counted and were defined as PAX7+ nuclei located within the basal lamina.

\section{Data analysis and statistics}

To address the relation between alterations in skeletal muscle mass- and oxidative metabolism-related processes during RL after disuse, clustering of human skeletal muscle biochemical and physiologic parameters was performed with the SciPy (v.0.19.1) toolkit (41). The RL response (RL-UL) per parameter was expressed as the percentage change from UL. The Spearman correlation coefficient between each pair of relative $\mathrm{RL}$ responses (i.e., $\mathrm{Rj}, \mathrm{k}$ ) was computed and subsequently transformed to Euclidean distance (Dj,k) with (Eq. 1):

$$
D_{j, k}=V\left(1-\left|R_{j, k}\right|\right)
$$


The resulting Euclidean distance matrix was hierarchically clustered based on the between-cluster average linkage using SciPy's cluster.hierarchy.linkage module and visualized using the clustermap module within Seaborn v.0.8.1 (42). Differences in the distribution of markers between clusters were tested by Pearson's $\chi 2$ test with post-hoc analysis based on adjusted residuals with Bonferroni correction.

The RL response per parameter of paired data (human biopsies) was tested by paired-samples t-test. The time-dependent reloading response (mouse muscle) was tested by 1-way ANOVA with Dunnett's post-hoc test, using UL as a reference group. Data are expressed as means \pm SEM, unless indicated otherwise. Statistical analyses were performed with SPSS Statistics (v.22.0; IBM, Armonk, NY, USA).

\section{Results}

One week of remobilization after leg immobilization-induced atrophy tended to increase quadriceps CSA (Table 1). However, neither quadriceps CSA nor muscle force measured by 1-RM had returned to preimmobilization baseline levels, indicating that recovery was still ongoing.

\section{Regulators and mediators of myogenesis, autophagy, and mitochondrial mass and dynamics correlate during recovery from muscle disuse in humans}

To address whether there is a correlation between alterations in skeletal muscle mass- and oxidative metabolism-related processes during RL after disuse, we measured the protein and mRNA expression of several regulators and mediators of protein turnover, mitochondrial turnover, and myonuclear turnover, before and after $7 \mathrm{~d}$ of RL in humans and clustered the correlations between relative $\mathrm{RL}$ responses based on similarity. From this, 4 clusters of parameters were identified (Figure 1).

Cluster 1 appeared enriched in markers of protein turnover signaling, whereas cluster 2 seemed to be enriched in markers of muscle mass and function (Table S3). Furthermore, two major clusters (clusters $3 \& 4$ ) occurred in which markers of myogenesis and autophagy were strongly represented (Table S3). This strong representation of myogenesis and autophagy markers was accompanied by a high percentage of markers of mitochondrial turnover and mitochondrial dynamics in cluster 3 , whereas cluster 4 was concomitantly enriched in markers of mitochondrial mass and appeared enriched in markers of mitochondrial dynamics, and to some extent, protein 


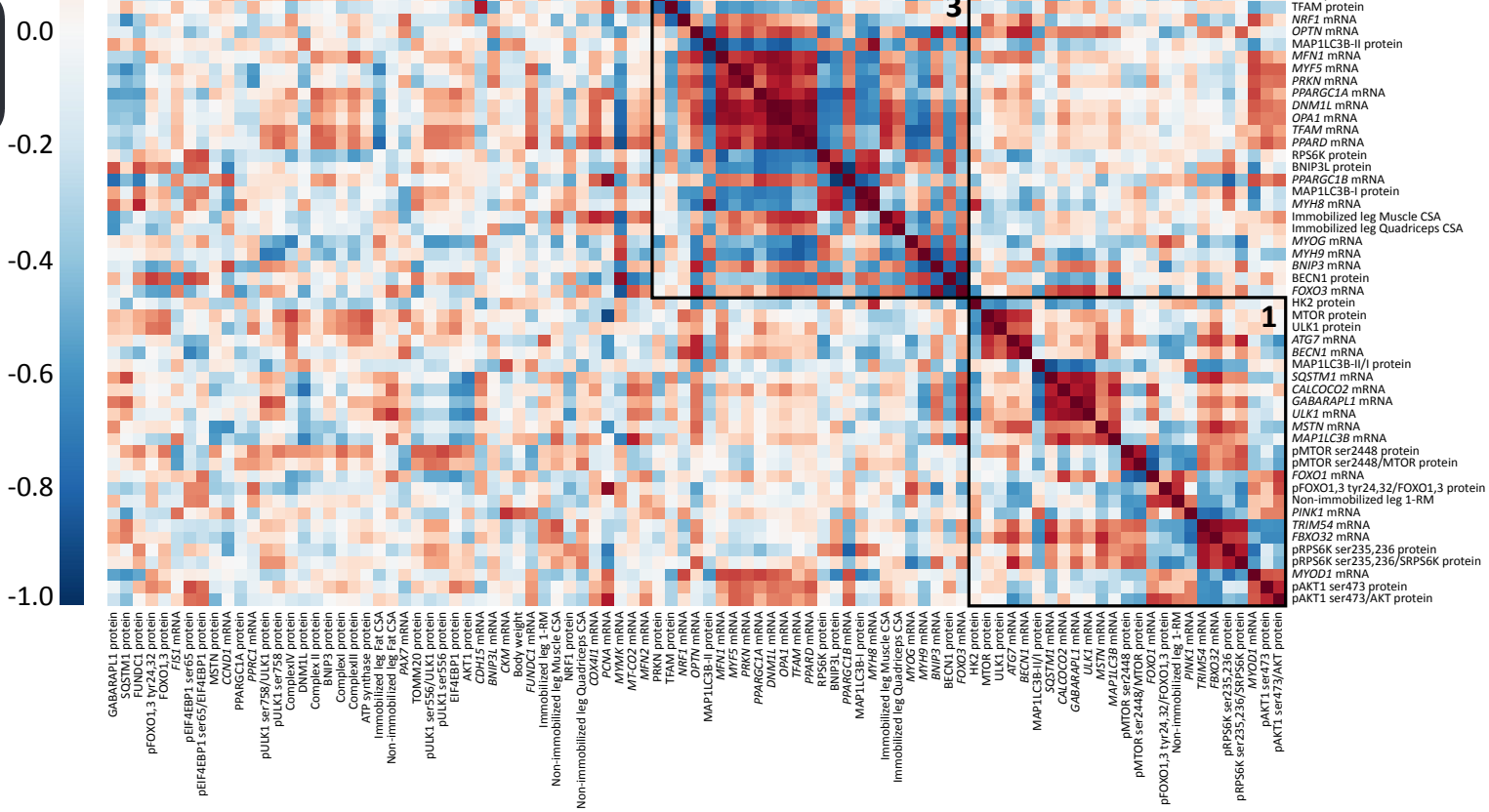

Figure 1. A correlation heatmap and cluster dendrogram of biochemical and functional parameters during skeletal muscle recovery from disuse in humans. Each square represents the Spearman correlation coefficient between the relative reloading responses of parameters displayed on the $x$ and $y$ axis, with colors indicating the strength and direction of the correlation. 
turnover (Table S3). Furthermore, most of the myogenesis markers represented in cluster 3 [i.e., myogenic factor (MYF)-5, myosin heavy chain (MYH)-8, myogenin (MYOG), and MYH9 mRNA] are associated with late stages of myogenesis (i.e., differentiation and fusion), whereas most of the myogenesis regulators and markers represented in cluster 4 [i.e., myostatin (MSTN) protein, and cyclin D1, PAX7, and cadherin $15 \mathrm{mRNA}$ ] are associated with early stages of myogenesis (i.e., proliferation). Moreover, most of the autophagy markers in cluster 3 [i.e., optine urin (OPTN) mRNA, and microtubule associated protein 1 light chain 3 b (MAP1LC3B)-II, MAP1LC3B-I, and beclin (BECN)-1 protein] are indicators of autophagosome formation, whereas most of the autophagy markers in cluster 4 [i.e., phosphorylated unc-51 like autophagy activating kinase 1 (ULK1) ser556, pULK1ser556:ULK1 ratio, pULK1ser758, pULK1ser758:ULK1 ratio, GABA type A receptor associated protein like 1 (GABARAPL1), and sequestosome 1 (SQSTM1) protein] are indicators of autophagy initiation.

\section{Induction of late myogenic markers after $\mathbf{5} \mathbf{d}$ of $\mathbf{R L}$ in mice}

The distinct clustering of early (cluster 4 ) and late (cluster 3 ) myogenesis markers upon remobilization in human subjects may reflect a time-dependent expression pattern of these markers, as previously observed for early myogenesis markers by Pansters et al. (30). This RL time-course mouse study was therefore used to address those time-dependent expression patterns. In this study, UL decreased the myofiber CSA, myonuclear domain, and number of nuclei per fiber (Figure $2 \mathrm{~A}-\mathrm{C}$ ). Ongoing recovery was indicated by an incomplete normalization of CSA and myonuclear domain up to $\mathrm{RL}-8$, and the number of nuclei per fiber was significantly restored at RL-3 and -8 . This restoration of the myonuclei was paralleled by a tendency toward an increased number of SCs at RL-3 (Figure 2D-E).

To assess whether late myogenesis markers indeed display a distinct temporal expression pattern, we measured markers of SC fusion and early myofiber regeneration in mouse gastrocnemius at $1,2,3,5$, and $8 \mathrm{~d}$ of $\mathrm{RL}$ after hindlimb suspension. At RL-1 and -2, Myh9 was transiently increased and gradually normalized at RL-5 and -8 (Figure 3). The expression of another, novel fusion marker, myomaker (Mymk), was strongly but transiently induced at RL-5. Furthermore, Myh8 was induced at RL-5 and -8. Expression of the musclespecific gene creatine kinase $(\mathrm{Ckm})$ was reduced upon UL, and was transiently further reduced at RL-1, -2 and -3 , whereas expression of the negative regulator of muscle mass Mstn was reduced at RL-5 and -8 . 
A

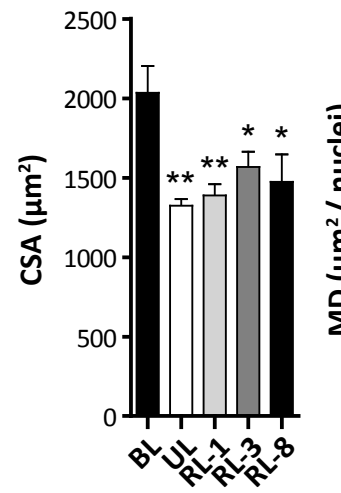

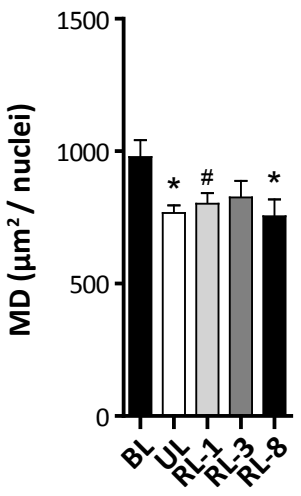

C

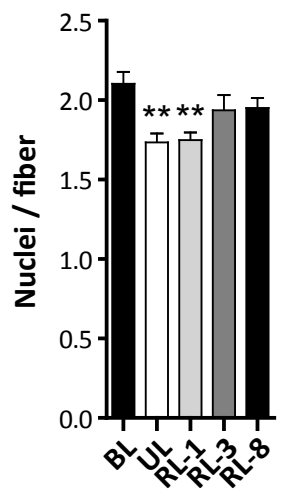

D

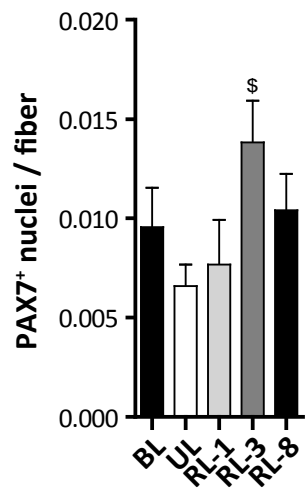

E

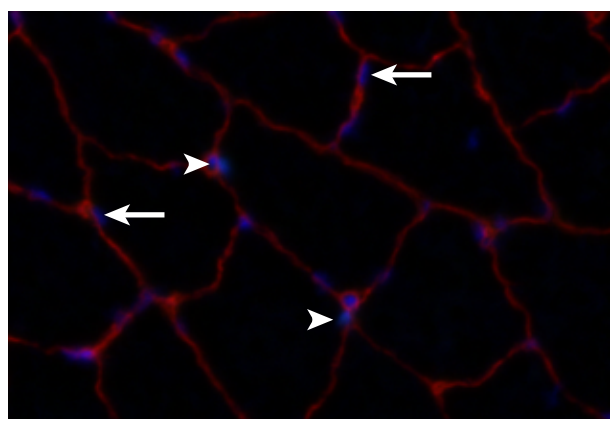

Laminin + PAX7

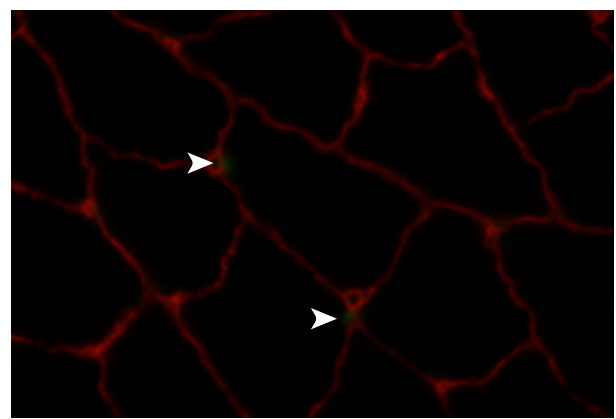

Figure 2. Changes in myofiber CSA, MD, myonuclear number, and satellite cell number during muscle disuse and recovery in mice. $M$. gastrocnemius muscle was collected at baseline (BL), after 14 days of unloading (UL), or after 1,3 , or 8 days of reloading (RL), for immunohistochemical determination of: A) Average muscle fiber cross-sectional area (CSA) ( $\mu \mathrm{m} 2)$. B) Myonuclear domain (MD) expressed as average $\mu \mathrm{m} 2 /$ myonucleus. C) Average number of myonuclei per muscle fiber. D) Average number of PAX7+ satellite cells per muscle fiber. E) Representation of immunohistochemical detection of Laminin (red) and PAX7 (green), and nuclei stained with DAPI (blue). Arrow heads indicate PAX7+ satellite cells, arrows indicate myofiber nuclei. ${ }^{\#} p<0.1, *^{*} p<0.05$, $* * p<0.01, * * * p<0.001$ compared to $\mathrm{BL},{ }^{\$} p<0.05$ compared to UL.

\section{Parallel modulation of autophagy and myogenesis in mice}

To address whether the temporal expression patterns of early and late myogenesis markers coincide with a temporal regulation of autophagy (as suggested from clusters 3 and 4), mRNA and protein levels of indicators of autophagosome formation and markers of autophagy capacity and autophagy initiation were measured.

The relative protein expression of MAP1LC3B-I displayed an acute but transient increase at RL-1, -2 and -3 , whereas MAP1LC3B-II levels remained largely unaltered (Figure 4). The MAP1LC3B-II:I ratio was decreased at RL-1, but subsequently normalized, and even increased at RL-8. Furthermore, the relative 


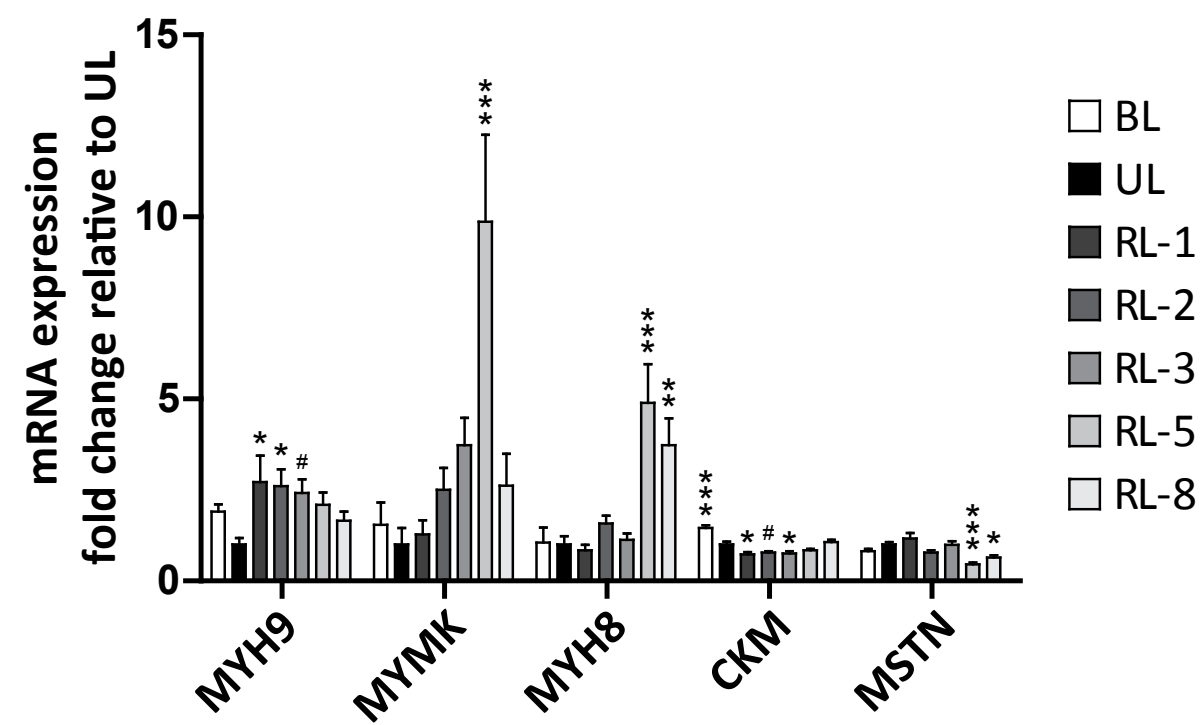

Figure 3. Temporal changes in mRNA expression of markers and mediators of late myogenesis upon recovery from muscle disuse in mice. mRNA expression of MYH9, MYMK, MYH8, CKM, and MSTN was assessed in $M$. gastrocnemius upon recovery from 14-days hindlimb suspension. UL, unloading; RL, reloading. ${ }^{\#} p<0.1,{ }^{*} p<0.05,{ }^{* *} p<0.01, * * * p<0.001$ compared to UL.

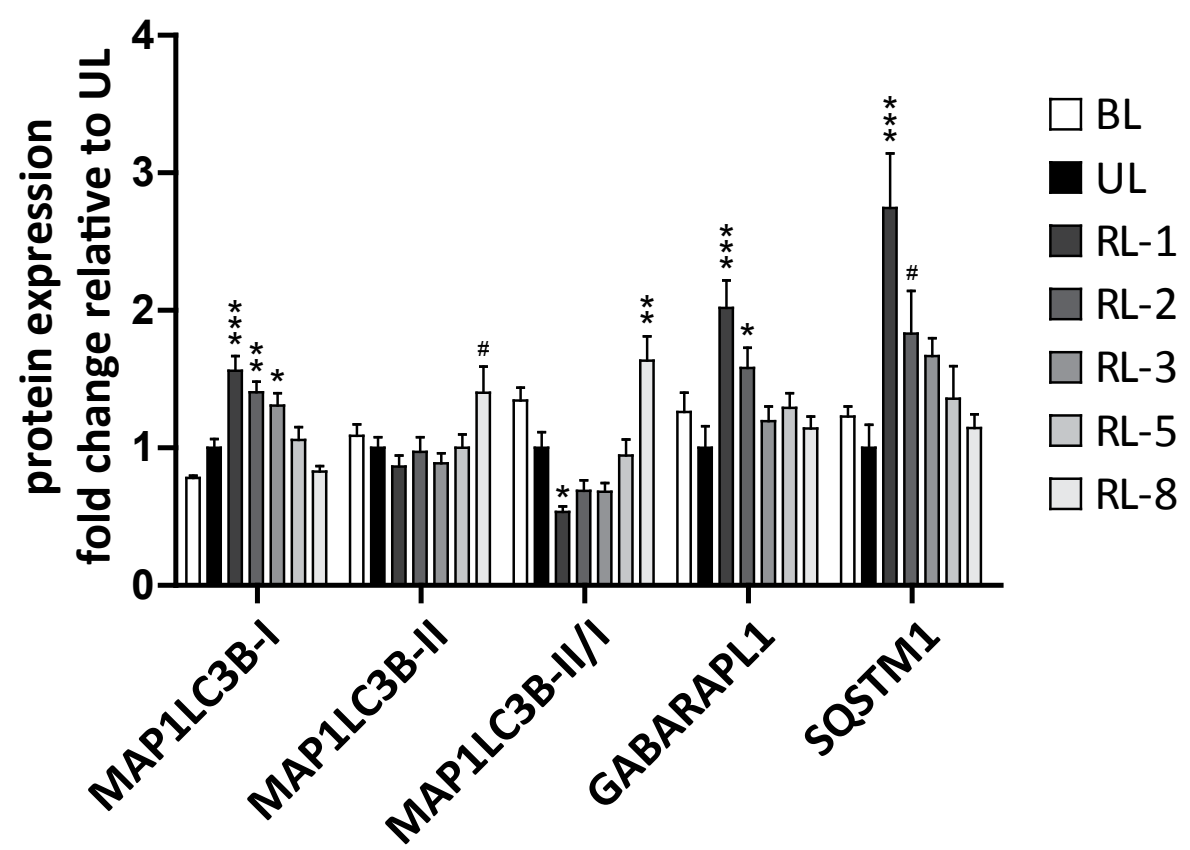

Figure 4. Temporal changes in protein expression of autophagosome formation indicators upon recovery from muscle disuse in mice. Protein expression of MAP1LC3B-I, MAP1LC3B-II, MAP1LC3B-II/I, GABARAPL1 and SQSTM1 was assessed in M. gastrocnemius upon recovery from 14-days hindlimb suspension. UL, unloading; RL, reloading. ${ }^{*} p<0.1,{ }^{*} p<0.05,{ }^{* *} p<0.01,{ }^{* * *} p<0.001$ compared to UL. 


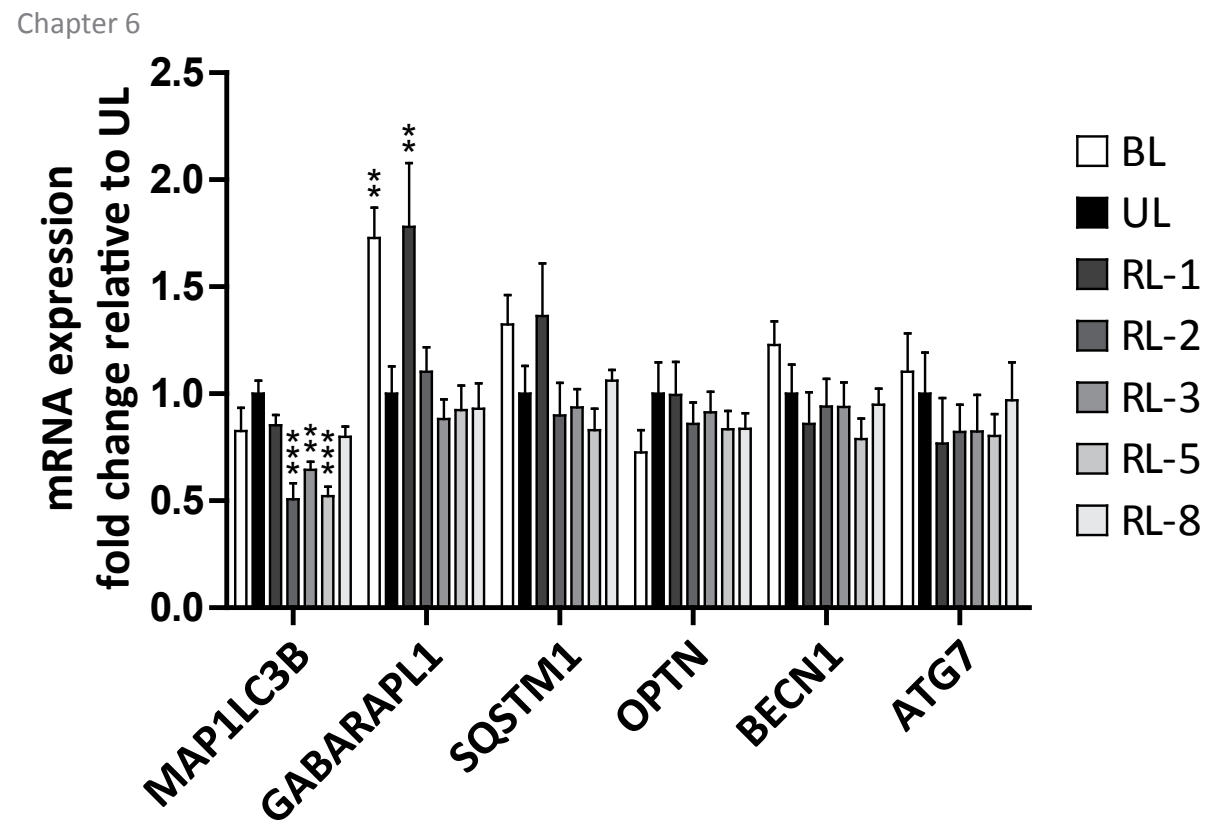

Figure 5. Temporal changes in mRNA expression of autophagy-related genes upon recovery from muscle disuse in mice. mRNA expression of MAP1LC3B, GABARAPL1, SQSTM1, OPTN, BECN1, and ATG7 was assessed in $m$. gastrocnemius upon recovery from 14-days hindlimb suspension. UL, unloading; RL, reloading. ${ }^{*} p<0.1,{ }^{* *} p<0.01,{ }^{* *} p<0.001$ compared to UL.

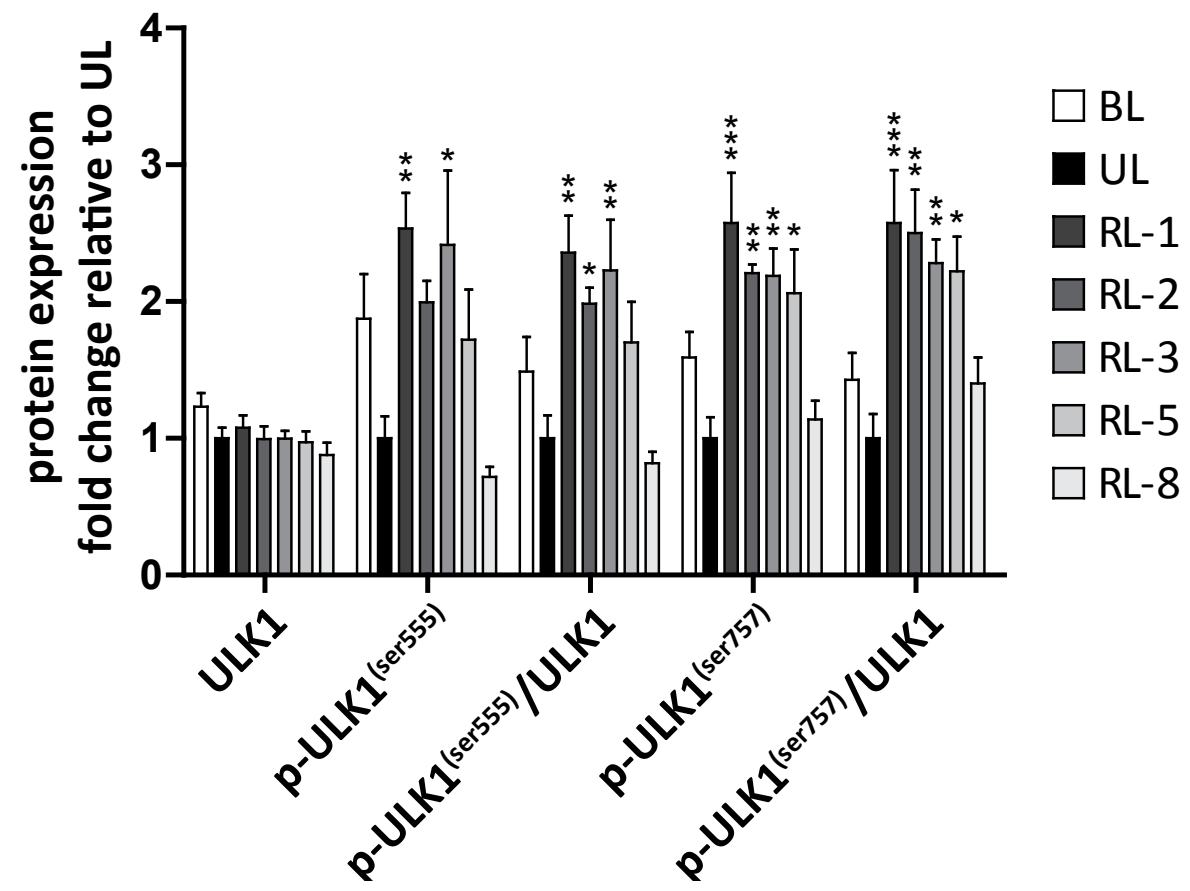

Figure 6. Temporal changes in protein expression of markers of autophagy initiation upon recovery from muscle disuse in mice. Protein expression of ULK1, p-ULK1(ser555), p-ULK1(ser555)/ULK1 ratio, p-ULK1(ser757), and p-ULK1(ser757)/ULK1 ratio was assessed in $m$. gastrocnemius upon recovery from 14-days hindlimb suspension. UL, unloading; RL, reloading. ${ }^{*} p<0.1,{ }^{*} p<0.05, * * p<0.01,{ }^{* * *} p<0.001$ compared to UL. 
mRNA expression of Map1/c3b was decreased at RL-2, -3 and -5 , and seemed to be partially normalized at RL-8 (Figure 5). In contrast, GABARAPL1 and SQSTM1 protein and mRNA expression displayed an acute but transient increase at RL-1 compared to UL, although Gabarap/1 mRNA was initially reduced upon UL (Figure 4, Figure 5). The mRNA expression of Optn, Becn1 and autophagy related-7 were unaltered during RL (Figure 5).

To further address a main regulatory step in the conversion of changes in autophagy capacity into flux, the activating and inhibiting phosphorylation of ULK1 was assessed. Both absolute and relative activating ULK1 phosphorylation (ser555) were increased during RL, up to RL-3 (Figure 6). However, in parallel, both absolute and relative inhibiting ULK1 phosphorylation (ser757) were increased during RL, up to RL-5.

\section{Temporal induction of mitophagy during recovery from disuse in mice}

Next to the predominance of markers of autophagosome formation in cluster 3 , this cluster also appeared to be enriched in markers of mitophagy. We therefore assessed the temporal expression of mitophagy markers during recovery from disuse in mice.

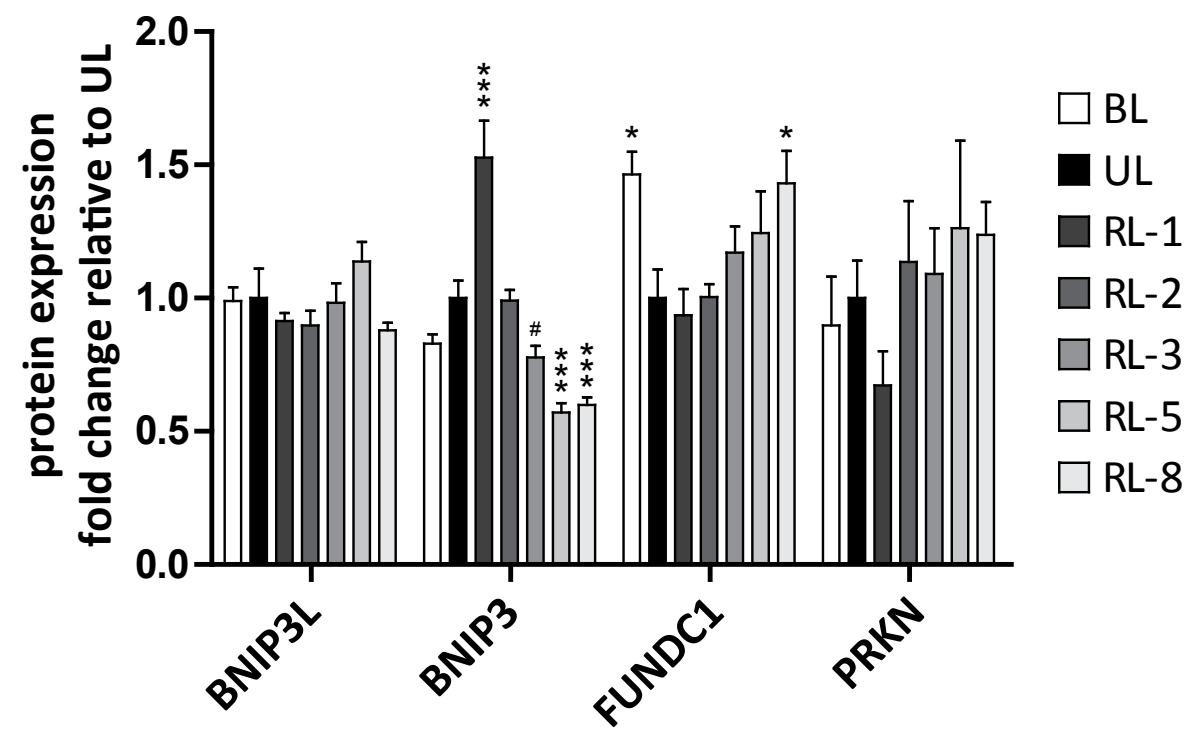

Figure 7. Temporal changes in protein expression of mitophagy regulators and mediators upon recovery from muscle disuse in mice. Protein expression of BNIP3L, BNIP3, FUNDC1, and PRKN was assessed in $m$. gastrocnemius upon recovery from 14-days hindlimb suspension. UL, unloading; RL, reloading. ${ }^{*} p<0.05,{ }^{* *} p<0.01,{ }^{* * *} p<0.001$ compared to UL. 


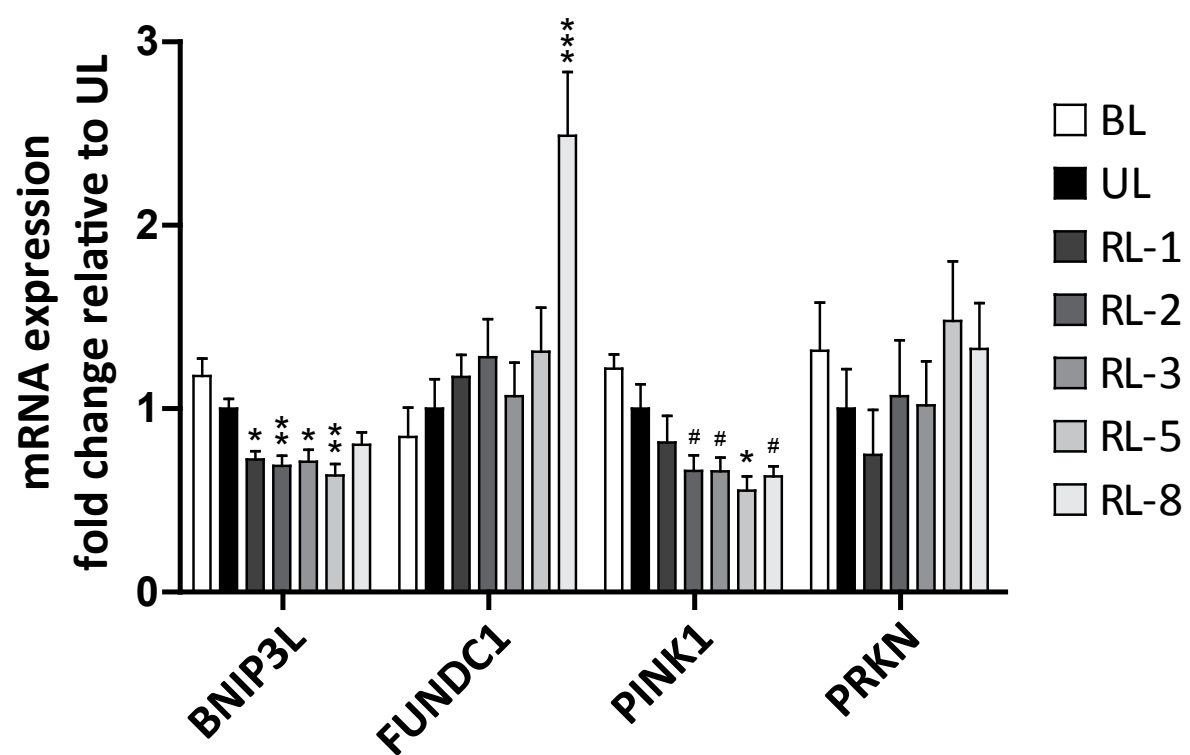

Figure 8. Temporal changes in mRNA expression of mitophagy-related genes upon recovery from muscle disuse in mice. mRNA expression of BNIP3L, BNIP3, FUNDC1, PINK1, and PRKN was assessed in $m$. gastrocnemius upon recovery from 14-days hindlimb suspension. UL, unloading; $\mathrm{RL}$, reloading. ${ }^{*} p<0.1,{ }^{* *} p<0.01,{ }^{* * *} p<0.001$ compared to UL.

No significant alterations in protein expression of BCL2 interacting protein 3 like (BNIP3L) or parkin RBR E3 ubiquitin protein ligase (PRKN) were observed during recovery after disuse. However, the protein expression of BNIP3 increased at $\mathrm{RL}-1$, subsequently returned to $\mathrm{UL}$ values, and decreased at RL-5 and -8 (Figure 7). Furthermore, FUN14 domain containing 1 (FUNDC1) protein expression was increased at RL-8 compared to UL, although an initial decrease was observed upon UL. Corresponding to its change in protein abundance, Fundc1 mRNA expression was increased at RL-8 (Figure 8). Furthermore, Bnip31 mRNA expression was decreased, and phosphatase and tensin homolog (PTEN)induced putative kinase 1 (Pink)-1 tended to be decreased upon remobilization, up to $d \mathrm{RL}-8$, but no changes in the expression of Prkn mRNA were observed.

\section{Discussion}

Alterations in the regulation of both muscle mass and metabolic plasticity during recovery from disuse have been reported, but the interaction between these processes is understudied. Hence, we explored this potential interaction by measuring established molecular regulators and mediators of processes that determine muscle mass and metabolism in human and mouse muscle and showed that molecular markers of myogenesis, autophagy, and mitochondrial mass and dynamics change coordinately during recovery from disuse. 
In the current human study, 1 wk of remobilization after leg immobilizationinduced atrophy increased quadriceps CSA compared to postimmobilization, but did not return quadriceps CSA or muscle force measured by 1-RM to preimmobilization baseline levels. This finding is in line with those in studies that showed an incomplete normalization of CSA $(43,44)$ and muscle strength (44) upon $1 \mathrm{wk}$ of recovery after immobilization and a complete normalization of muscle mass and strength after 2 wk of recovery $(25,44-47)$. This result shows that after 1 wk of remobilization, skeletal muscle recovery is still ongoing, and thus allows us to study the processes that are associated with recovery.

We detected substantial interindividual variation in the human molecular responses to RL (Figure S1), which was not affected by correction for creatine supplementation. We exploited this variation to assess the relation between molecular RL responses. Based on hierarchical cluster analysis of the correlations between relative RL responses, we identified 4 clusters of parameters. The coappearance of markers and mediators of classic muscle mass- and oxidative metabolism-related processes within clusters 3 and 4 may point at an interdependency or joint regulation of these processes during recovery from atrophy. Although we cannot exclude creatine supplementation as a potential confounding factor and a modulatory effect of creatine supplementation on certain molecular processes has been suggested $(48,49)$, there is no indication of an effect of creatine supplementation on the interrelationship between the regulatory processes associated with recovery from muscle atrophy, and correction for creatine supplementation does not alter the pattern of correlations between RL effects. Markers that regulate and mediate different stages of myogenesis and autophagy displayed a distinct clustering, which may be related to differences in their temporal expression patterns. Between-subject asynchronicity in such temporal expression patterns may contribute to the observed variation, which - despite its applicability in the assessment of between-variable associations - limits the statistical power to detect relevant changes on a group level. Furthermore, interindividual asynchronicity, combined with a single time-point observation during recovery in humans, complicates the synthesis of a univocal conclusion on the molecular RL response. The high-resolution time-course study in mice was thus instrumental in the indepth exploration of the time-dependent molecular regulation of muscle mass and metabolic plasticity during disuse-induced atrophy recovery.

Similar to the study in humans, mice displayed an incomplete normalization of muscle weight (30) and muscle fiber CSA, which supports ongoing recovery over $8 \mathrm{~d}$ of RL. Three days of RL after hindlimb suspension transiently increased the number of PAX7+ SCs per fiber. This finding is in line with previous studies 
assessing SC numbers after relative short-term remobilization in rodents (50-52) and humans (46) and may explain the unaltered number of SCs upon longer term remobilization (53). In accordance with the early induction of SC proliferation, our lab showed in another study an early and potentially biphasic induction of markers and mediators of the initial steps in myogenesis in this model (30). In the current work, we showed for the first time that, subsequent to this increase in early myogenesis markers, the markers and mediators of late stages of myogenesis Mymk and Myh8 are increased toward the end of the $8 \mathrm{~d}$ RL phase. Furthermore, in line with previous studies (54-56), there was a partial restoration of the number of nuclei per fiber after $3 \mathrm{~d}$ of RL, which supports the notion that SCs functionally contribute to skeletal muscle recovery after disuse (57). Indeed, because the myonuclear domain was unaltered during remobilization, the increase in myonuclear content is expected to result in an increase in muscle fiber CSA. However, as this change in fiber CSA is not yet significantly detectable, we conclude that muscle fiber CSA is incompletely restored up to $8 \mathrm{~d}$ of remobilization. These findings could be speculated to result from a lag time between myonuclear accretion and a functional contribution of the newly incorporated nucleus to protein synthesis or to reflect a role for myonuclear accretion in muscle fiber metabolic plasticity. Despite the incomplete normalization of muscle fiber CSA and myonuclear domain over the time course examined in this mouse study, their recovery seemed to start as early as RL-1. This is in agreement with the previously observed acute and sustained increase in protein synthesis signaling $(30,58-60)$ and increased protein synthesis rates upon RL (61). This anabolic state precedes myonuclear accretion, which supports the notion that hypertrophy can occur to some extent independent of SCs (62). Furthermore, previous studies have consistently shown a suppression of mediators of the UPS during recovery from disuse $(27,30,44,52,59,60,63-65)$, whereas a transient increase in proteasome activity $(27,61)$ and ubiquitin-conjugated proteins $(60)$ have been observed simultaneously.

In this study, we conducted a comprehensive analysis of markers of autophagosome formation, autophagy capacity, and autophagy initiation. From this, we conclude that autophagy is actively regulated both acutely, as well as after 5 and $8 \mathrm{~d}$ of RL, which appears to parallel the temporal regulation of myonuclear accretion. We showed an acute but transient increase in the autophagosome formation markers MAP1LC3B-I, GABARAPL1 and SQSTM1 protein, and a decrease and subsequent increase in the MAP1LC3B-II:I ratio that parallels the regulation of late stages of myogenesis. In line with our data, previous studies showed an increase in Sqstm1 protein expression (27) and an increase in MAP1LC3B-II:I ratio (31). In addition, White et al. (63) reported an unaltered MAP1LC3B-II:I 
ratio after $14 \mathrm{~d}$ of $\mathrm{RL}$, potentially reflecting a normalization of autophagy signaling upon completed recovery. Furthermore, in line with previous studies $(59,66)$, we showed a transient decrease in the mRNA expression of the autophagy capacity indicator Map1/c3b and a transient increase in the mRNA expression of Gabarap/1 relative to UL. In addition to the reported increase in ULK1 inhibiting phosphorylation $(27,29)$, we report a concomitant induction of ULK1 inhibiting and activating phosphorylation. This temporal regulation of autophagy initiation resembles the expression pattern of early myogenesis markers observed by Pansters et al. (30).

Despite our comprehensive analysis of autophagy regulation, we did not measure autophagy flux and therefore cannot draw an unambiguous conclusion regarding the net effect of RL on the autophagy flux itself. Furthermore, alterations in expression levels of several autophagy regulators and mediators seem contradictory, which emphasizes the complexity of autophagy regulation during recovery after disuse. We speculate that this complexity reflects the degradation of specific targets (e.g., mitochondria), which is in accordance with a possible involvement of autophagy in skeletal muscle metabolic plasticity in addition to its classic role in muscle mass regulation $(67,68)$. Recent papers suggest a crucial role for autophagy, and more specifically, mitophagy, in skeletal muscle functional regeneration $(69,70)$.

During recovery after disuse in mice, the gene expression of the mitochondrial damage-related mitophagy regulator Pink1 tends to be decreased, whereas Prkn is unaltered. In addition, PRKN protein expression is unaltered during remobilization, although it should be noted that this mitophagy pathway is also modulated by posttranslational modifications (71), which were not assessed in this study. Receptor-mediated mitophagy via BNIP3L, BNIP3, and FUNDC1 can be induced by both hypoxia and increased oxidative stress (72), of which an increase during $\mathrm{RL}$ has been documented $(73,74)$. BNIP3 protein expression transiently increased, and subsequently decreased during recovery, which parallels the previously reported alterations in Bnip3 mRNA expression in this mouse study (30), and the increase in Bnip3 protein expression reported by Kang and Ji (74). In contrast, BNIP3L protein expression is unaltered upon recovery after disuse, whereas Bnip3/ mRNA expression displays a persistent decrease up to RL-5. The mRNA and protein expression of Fundc1 is specifically induced at $\mathrm{RL}-8$, suggesting a late-phase response.

These molecular alterations point toward a temporal induction of mitophagy both acutely and after $8 \mathrm{~d}$ of recovery after disuse. Nevertheless, previous data suggest a normalization of mitochondrial content upon several days of RL 
$(34,73-75)$, likely driven by an increase in mitochondrial biogenesis $(34,73)$. The induction of mitophagy in the presence of a recovery of mitochondrial content suggests the induction of mitochondrial remodeling during remobilization. Indeed, an increase in mitochondrial remodeling (75) and mitochondrial dynamics $(34,75)$ has been observed during recovery from disuse. This RL-induced mitochondrial remodeling may be a response to increased oxphos (76) and could serve to meet the altered metabolic demands during remobilization. Another observation is that a late increase in Fundc1 expression paralleled the temporal regulation of late-stage myogenesis markers. It is becoming increasingly clear that mitophagy (77), mitochondrial dynamics $(78,79)$, and metabolic reprogramming (80-83) are crucial for myogenic differentiation. Furthermore, Mishra et al. (76) showed that mitochondrial proteins compartmentalize to discrete domains located around a nucleus of origin that originates from SC-mediated myonuclear accretion. In line, myogenesis seems to be involved in the aerobic adaptation in response to endurance-type exercise (84). Clearly, this indicates a close relation between muscle mass- and oxidative metabolism-related processes.

\section{Conclusion}

We showed, through the combination of a cluster analysis and high-resolution time-course study, that the molecular regulation of protein turnover, mitochondrial turnover, and myonuclear turnover are correlated and temporally associated during recovery from atrophy. This result suggests an interdependency or joint regulation of these muscle mass- and oxidative metabolism-related processes during recovery from atrophy and emphasizes the need to study these processes by integrated molecular analyses. 


\section{Acknowledgements}

The authors thank Marco Kelders, Chiel de Theije, and Antoine Zorenc for their excellent technical assistance in performing the molecular analyses, and Roy Haast for his support in conducting the cluster analysis. The original work from which human muscle biopsies were obtained [36] was funded by TI Food and Nutrition, a public-private partnership on precompetitive research in food and nutrition. The mouse study was funded by a grant from the Lung Foundation/Netherlands Asthma Foundation (NAF 3.2.07.017) and the transnational University Limburg (tUL). For the current work, the researchers had sole responsibility for the study design, data collection and analysis, decision to publish, and preparation of the manuscript. The authors declare no conflicts of interest. 


\section{References}

1. Egan B, Zierath JR. Exercise metabolism and the molecular regulation of skeletal muscle adaptation. Cell Metab 2013;17(2):162-84.

2. Wall BT, van Loon LJ. Nutritional strategies to attenuate muscle disuse atrophy. Nutr Rev 2013;71(4):195-208.

3. Wall BT, Dirks ML, Snijders T, et al. Substantial skeletal muscle loss occurs during only 5 days of disuse. Acta Physiol (Oxf) 2014;210(3):600-11.

4. Brooks NE, Myburgh KH. Skeletal muscle wasting with disuse atrophy is multidimensional: the response and interaction of myonuclei, satellite cells and signaling pathways. Frontiers in physiology 2014;5:99.

5. Bierbrauer J, Koch S, Olbricht C, et al. Early type II fiber atrophy in intensive care unit patients with nonexcitable muscle membrane. Crit Care Med 2012;40(2):647-50.

6. Pette D, Staron RS. Transitions of muscle fiber phenotypic profiles. Histochem Cell Biol 2001;115(5):359-72.

7. Yu ZB, Gao F, Feng HZ, et al. Differential regulation of myofilament protein isoforms underlying the contractility changes in skeletal muscle unloading. Am J Physiol Cell Physiol 2007;292(3):C1192-203.

8. Stein TP, Wade CE. Metabolic consequences of muscle disuse atrophy. J Nutr 2005;135(7):1824S-28S.

9. Gram M, Dahl R, Dela F. Physical inactivity and muscle oxidative capacity in humans. Eur J Sport Sci 2014;14(4):376-83.

10. Dirks ML, Wall BT, van de Valk B, et al. One Week of Bed Rest Leads to Substantial Muscle Atrophy and Induces Whole-Body Insulin Resistance in the Absence of Skeletal Muscle Lipid Accumulation. Diabetes 2016;65(10):2862-75.

11. Gram M, Vigelso A, Yokota $\mathrm{T}$, et al. Skeletal muscle mitochondrial $\mathrm{H} 2 \mathrm{O} 2$ emission increases with immobilization and decreases after aerobic training in young and older men. J Physiol 2015;593(17):4011-27.

12. Romanello V, Guadagnin E, Gomes L, et al. Mitochondrial fission and remodelling contributes to muscle atrophy. EMBO J 2010;29(10):1774-85.

13. Seene $T$, Kaasik P, Riso EM. Review on aging, unloading and reloading: changes in skeletal muscle quantity and quality. Arch Gerontol Geriatr 2012;54(2):374-80.

14. Rizzoli R, Reginster JY, Arnal JF, et al. Quality of life in sarcopenia and frailty. Calcif Tissue Int 2013;93(2):101-20.

15. Schols AM, Broekhuizen R, Weling-Scheepers CA, et al. Body composition and mortality in chronic obstructive pulmonary disease. Am J Clin Nutr 2005;82(1):53-9.

16. Martin L, Birdsell L, Macdonald N, et al. Cancer cachexia in the age of obesity: skeletal muscle depletion is a powerful prognostic factor, independent of body mass index. J Clin Oncol 2013;31(12):1539-47.

17. Marzetti E, Calvani R, Tosato M, et al. Sarcopenia: an overview. Aging Clin Exp Res 2017;29(1):11-17.

18. Dirks ML, Wall BT, Snijders T, et al. Neuromuscular electrical stimulation prevents muscle disuse atrophy during leg immobilization in humans. Acta Physiol (Oxf) 2014;210(3):628-41.

19. Dirks ML, Wall BT, Nilwik R, et al. Skeletal muscle disuse atrophy is not attenuated by dietary protein supplementation in healthy older men. J Nutr 2014;144(8):1196-203. 
20. Ferrando AA, Paddon-Jones D, Hays NP, et al. EAA supplementation to increase nitrogen intake improves muscle function during bed rest in the elderly. Clin Nutr 2010;29(1):18-23.

21. Paddon-Jones D, Sheffield-Moore M, Urban RJ, et al. Essential amino acid and carbohydrate supplementation ameliorates muscle protein loss in humans during 28 days bedrest. J Clin Endocrinol Metab 2004;89(9):4351-8.

22. Reidy PT, McKenzie Al, Brunker P, et al. Neuromuscular Electrical Stimulation Combined with Protein Ingestion Preserves Thigh Muscle Mass But Not Muscle Function in Healthy Older Adults During 5 Days of Bed Rest. Rejuvenation Res 2017

23. Dideriksen K, Boesen AP, Kristiansen JF, et al. Skeletal muscle adaptation to immobilization and subsequent retraining in elderly men: No effect of antiinflammatory medication. Exp Gerontol 2016;82:8-18.

24. Tanner RE, Brunker LB, Agergaard J, et al. Age-related differences in lean mass, protein synthesis and skeletal muscle markers of proteolysis after bed rest and exercise rehabilitation. J Physiol 2015;593(18):4259-73.

25. Suetta C, Hvid LG, Justesen L, et al. Effects of aging on human skeletal muscle after immobilization and retraining. Journal of applied physiology 2009;107(4):1172-80.

26. Joanisse S, Nederveen JP, Snijders T, et al. Skeletal Muscle Regeneration, Repair and Remodelling in Aging: The Importance of Muscle Stem Cells and Vascularization. Gerontology 2017;63(1):91-100.

27. Baehr LM, West DW, Marcotte G, et al. Age-related deficits in skeletal muscle recovery following disuse are associated with neuromuscular junction instability and ER stress, not impaired protein synthesis. Aging (Albany NY) 2016;8(1):127-46.

28. Theriault $M E$, Pare $M E$, Lemire $B B$, et al. Regenerative defect in vastus lateralis muscle of patients with chronic obstructive pulmonary disease. Respir Res 2014;15:35.

29. Kelleher AR, Pereira SL, Jefferson LS, et al. REDD2 expression in rat skeletal muscle correlates with nutrient-induced activation of mTORC1: responses to aging, immobilization, and remobilization. Am J Physiol Endocrinol Metab 2015;308(2):E122-9.

30. Pansters NA, Schols AM, Verhees KJ, et al. Muscle-specific GSK-3beta ablation accelerates regeneration of disuse-atrophied skeletal muscle. Biochim Biophys Acta 2015;1852(3):490-506.

31. Takahashi H, Suzuki Y, Mohamed JS, et al. Epigallocatechin-3-gallate increases autophagy signaling in resting and unloaded plantaris muscles but selectively suppresses autophagy protein abundance in reloaded muscles of aged rats. Exp Gerontol 2017;92:56-66.

32. Kang C, Yeo D, Ji LL. Muscle immobilization activates mitophagy and disrupts mitochondrial dynamics in mice. Acta Physiol (Oxf) 2016;218(3):188-97.

33. McClung JM, Davis JM, Wilson MA, et al. Estrogen status and skeletal muscle recovery from disuse atrophy. Journal of applied physiology 2006;100(6):2012-23.

34. Remels $A H$, Pansters NA, Gosker HR, et al. Activation of alternative NF-kappaB signaling during recovery of disuse-induced loss of muscle oxidative phenotype. Am J Physiol Endocrinol Metab 2014;306(6):E615-26.

35. Lopez-Lluch G. Essential role of mitochondrial dynamics in muscle physiology. Acta Physiol (Oxf) 2017;219(1):20-21. 
36. Backx EM, Hangelbroek R, Snijders T, et al. Creatine Loading Does Not Preserve Muscle Mass or Strength During Leg Immobilization in Healthy, Young Males: A Randomized Controlled Trial. Sports Med 2017

37. Bodine SC, Latres $\mathrm{E}$, Baumhueter $\mathrm{S}$, et al. Identification of ubiquitin ligases required for skeletal muscle atrophy. Science 2001;294(5547):1704-8.

38. Ferreira R, Neuparth MJ, Ascensao A, et al. Skeletal muscle atrophy increases cell proliferation in mice gastrocnemius during the first week of hindlimb suspension. Eur J Appl Physiol 2006;97(3):340-6.

39. Hanson $\mathrm{AM}$, Harrison $\mathrm{BC}$, Young $\mathrm{MH}$, et al. Longitudinal characterization of functional, morphologic, and biochemical adaptations in mouse skeletal muscle with hindlimb suspension. Muscle Nerve 2013;48(3):393-402.

40. Ruijter JM, Thygesen $\mathrm{HH}$, Schoneveld OJ, et al. Factor correction as a tool to eliminate between-session variation in replicate experiments: application to molecular biology and retrovirology. Retrovirology 2006;3:2.

41. Oliphant TE. Python for scientific computing. Comput Sci Eng 2007;9(3):10-20.

42. mwaskom/seaborn: v0.8.1 (September 2017) [program]. September 3 version. https://doi.org/10.5281/zenodo.883859, 2017.

43. Hvid LG, Suetta C, Nielsen JH, et al. Aging impairs the recovery in mechanical muscle function following 4 days of disuse. Exp Gerontol 2014;52:1-8.

44. Jones SW, Hill RJ, Krasney PA, et al. Disuse atrophy and exercise rehabilitation in humans profoundly affects the expression of genes associated with the regulation of skeletal muscle mass. FASEB journal : official publication of the Federation of American Societies for Experimental Biology 2004;18(9):1025-7.

45. Hortobagyi T, Dempsey L, Fraser D, et al. Changes in muscle strength, muscle fibre size and myofibrillar gene expression after immobilization and retraining in humans. J Physiol 2000;524 Pt 1:293-304.

46. Suetta C, Frandsen U, Mackey AL, et al. Ageing is associated with diminished muscle re-growth and myogenic precursor cell expansion early after immobilityinduced atrophy in human skeletal muscle. J Physiol 2013;591(15):3789-804.

47. Hespel P, Op't Eijnde B, Van Leemputte $M$, et al. Oral creatine supplementation facilitates the rehabilitation of disuse atrophy and alters the expression of muscle myogenic factors in humans. J Physiol 2001;536(Pt 2):625-33.

48. Kim J, Lee J, Kim S, et al. Role of creatine supplementation in exercise-induced muscle damage: A mini review. J Exerc Rehabil 2015;11(5):244-50.

49. Deldicque $L$, Atherton $P$, Patel $R$, et al. Effects of resistance exercise with and without creatine supplementation on gene expression and cell signaling in human skeletal muscle. Journal of applied physiology 2008;104(2):371-8.

50. Stevens-Lapsley JE, Ye F, Liu M, et al. Impact of viral-mediated IGF-I gene transfer on skeletal muscle following cast immobilization. Am J Physiol Endocrinol Metab 2010;299(5):E730-40.

51. Ye $F$, Mathur S, Liu M, et al. Overexpression of insulin-like growth factor-1 attenuates skeletal muscle damage and accelerates muscle regeneration and functional recovery after disuse. Exp Physiol 2013;98(5):1038-52.

52. Park S, Brisson BK, Liu M, et al. Mature IGF-I excels in promoting functional muscle recovery from disuse atrophy compared with pro-IGF-IA. Journal of applied physiology 2014;116(7):797-806. 
53. Jackson JR, Mula J, Kirby TJ, et al. Satellite cell depletion does not inhibit adult skeletal muscle regrowth following unloading-induced atrophy. Am J Physiol Cell Physiol 2012;303(8):C854-61.

54. Mozdziak PE, Pulvermacher PM, Schultz E. Muscle regeneration during hindlimb unloading results in a reduction in muscle size after reloading. Journal of applied physiology 2001;91(1):183-90.

55. Wang XD, Kawano F, Matsuoka $Y$, et al. Mechanical load-dependent regulation of satellite cell and fiber size in rat soleus muscle. Am J Physiol Cell Physiol 2006;290(4):C981-9.

56. Mitchell PO, Pavlath GK. Skeletal muscle atrophy leads to loss and dysfunction of muscle precursor cells. Am J Physiol Cell Physiol 2004;287(6):C1753-62.

57. Mitchell PO, Pavlath GK. A muscle precursor cell-dependent pathway contributes to muscle growth after atrophy. Am J Physiol Cell Physiol 2001;281(5):C1706-15.

58. Washington TA, White JP, Davis JM, et al. Skeletal muscle mass recovery from atrophy in IL-6 knockout mice. Acta Physiol (Oxf) 2011;202(4):657-69.

59. Smith HK, Matthews KG, Oldham JM, et al. Translational signalling, atrogenic and myogenic gene expression during unloading and reloading of skeletal muscle in myostatin-deficient mice. PLoS One 2014;9(4):e94356.

60. Kramerova I, Kudryashova E, Venkatraman G, et al. Calpain 3 participates in sarcomere remodeling by acting upstream of the ubiquitin-proteasome pathway. Hum Mol Genet 2005;14(15):2125-34.

61. Lang SM, Kazi AA, Hong-Brown L, et al. Delayed recovery of skeletal muscle mass following hindlimb immobilization in mTOR heterozygous mice. PLoS One 2012;7(6):e38910.

62. McCarthy JJ, Mula J, Miyazaki M, et al. Effective fiber hypertrophy in satellite cell-depleted skeletal muscle. Development 2011;138(17):3657-66.

63. White JR, Confides AL, Moore-Reed S, et al. Regrowth after skeletal muscle atrophy is impaired in aged rats, despite similar responses in signaling pathways. Exp Gerontol 2015;64:17-32.

64. Andrianjafiniony $\mathrm{T}$, Dupre-Aucouturier $\mathrm{S}$, Letexier $\mathrm{D}$, et al. Oxidative stress, apoptosis, and proteolysis in skeletal muscle repair after unloading. Am J Physiol Cell Physiol 2010;299(2):C307-15.

65. Chacon-Cabrera A, Lund-Palau H, Gea J, et al. Time-Course of Muscle Mass Loss, Damage, and Proteolysis in Gastrocnemius following Unloading and Reloading: Implications in Chronic Diseases. PLoS One 2016;11(10):e0164951.

66. Slimani L, Micol D, Amat J, et al. The worsening of tibialis anterior muscle atrophy during recovery post-immobilization correlates with enhanced connective tissue area, proteolysis, and apoptosis. Am J Physiol Endocrinol Metab 2012;303(11):E1335-47.

67. Ferraro E, Giammarioli AM, Chiandotto S, et al. Exercise-induced skeletal muscle remodeling and metabolic adaptation: redox signaling and role of autophagy. Antioxid Redox Signal 2014;21(1):154-76.

68. Kaur J, Debnath J. Autophagy at the crossroads of catabolism and anabolism. Nat Rev Mol Cell Biol 2015;16(8):461-72.

69. Call JA, Wilson RJ, Laker RC, et al. Ulk1-mediated autophagy plays an essential role in mitochondrial remodeling and functional regeneration of skeletal muscle. Am J Physiol Cell Physiol 2017;312(6):C724-C32. 
70. Nichenko AS, Southern WM, Atuan M, et al. Mitochondrial maintenance via autophagy contributes to functional skeletal muscle regeneration and remodeling. Am J Physiol Cell Physiol 2016;311(2):C190-200.

71. Durcan TM, Fon EA. The three 'P's of mitophagy: PARKIN, PINK1, and posttranslational modifications. Genes \& development 2015;29(10):989-99.

72. Wu H, Chen Q. Hypoxia activation of mitophagy and its role in disease pathogenesis. Antioxid Redox Signal 2015;22(12):1032-46.

73. Kang C, Goodman CA, Hornberger TA, et al. PGC-1alpha overexpression by in vivo transfection attenuates mitochondrial deterioration of skeletal muscle caused by immobilization. FASEB journal : official publication of the Federation of American Societies for Experimental Biology 2015;29(10):4092-106.

74. Kang C, Ji LL. PGC-1alpha overexpression via local transfection attenuates mitophagy pathway in muscle disuse atrophy. Free Radic Biol Med 2016;93:32-40.

75. Liu J, Peng Y, Feng Z, et al. Reloading functionally ameliorates disuse-induced muscle atrophy by reversing mitochondrial dysfunction, and similar benefits are gained by administering a combination of mitochondrial nutrients. Free Radic Biol Med 2014;69:116-28.

76. Mishra P, Varuzhanyan G, Pham AH, et al. Mitochondrial Dynamics is a Distinguishing Feature of Skeletal Muscle Fiber Types and Regulates Organellar Compartmentalization. Cell Metab 2015;22(6):1033-44.

77. Sin J, Andres AM, Taylor DJ, et al. Mitophagy is required for mitochondrial biogenesis and myogenic differentiation of C2C12 myoblasts. Autophagy 2016;12(2):369-80.

78. Kim B, Kim JS, Yoon $Y$, et al. Inhibition of Drp1-dependent mitochondrial division impairs myogenic differentiation. Am J Physiol Regul Integr Comp Physiol 2013;305(8):R927-38.

79. Barbieri E, Battistelli M, Casadei L, et al. Morphofunctional and Biochemical Approaches for Studying Mitochondrial Changes during Myoblasts Differentiation. J Aging Res 2011;2011:845379.

80. Koopman R, Ly CH, Ryall JG. A metabolic link to skeletal muscle wasting and regeneration. Frontiers in physiology 2014;5:32.

81. Ceco E, Weinberg SE, Chandel NS, et al. Metabolism and Skeletal Muscle Homeostasis in Lung Disease. Am J Respir Cell Mol Biol 2017;57(1):28-34.

82. Fortini $P$, Ferretti $C$, lorio $E$, et al. The fine tuning of metabolism, autophagy and differentiation during in vitro myogenesis. Cell Death Dis 2016;7:e2168.

83. Fortini P, lorio E, Dogliotti E, et al. Coordinated Metabolic Changes and Modulation of Autophagy during Myogenesis. Frontiers in physiology 2016;7:237.

84. Abreu P, Mendes SV, Ceccatto VM, et al. Satellite cell activation induced by aerobic muscle adaptation in response to endurance exercise in humans and rodents. Life Sci 2017;170:33-40. 


\section{Supplemental information}

A

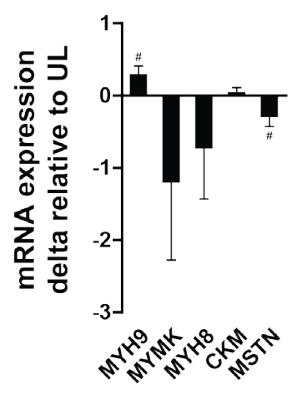

$E$

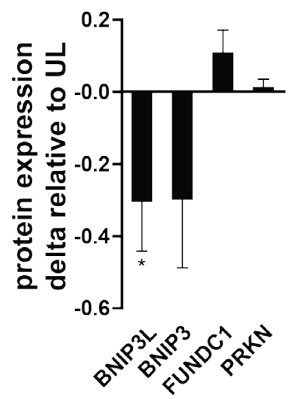

B

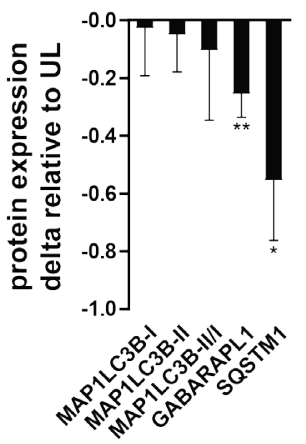

F

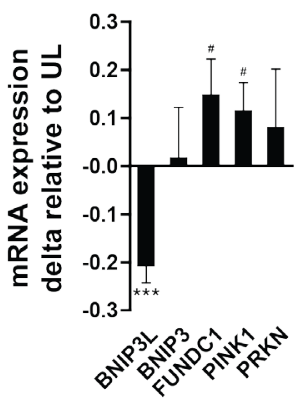

C

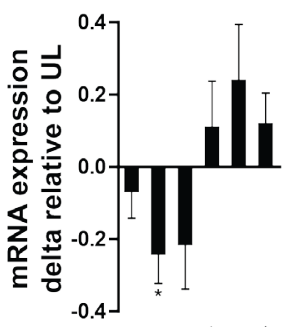

के

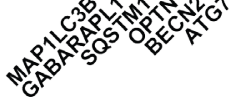

D
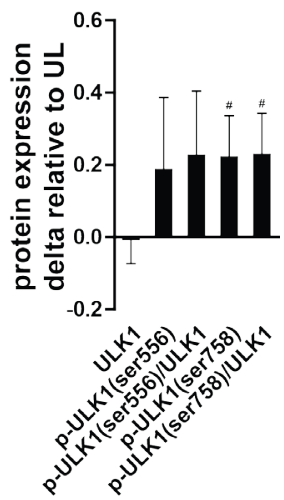

Figure S1: Change in mRNA and protein expression in human $m$. vastus lateralis upon 1-week recovery from disuse. Relative change in mRNA and protein expression upon reloading (RL), in fold change compared to unloading (UL). A. Relative mRNA expression of markers and mediators of late myogenesis. B. Relative protein expression of indicators of autophagosome formation. C. Relative mRNA expression of autophagy-related genes. D. Relative protein expression of markers of autophagy initiation. E. Relative protein expression of mitophagy regulators and mediators. F. Relative mRNA expression of mitophagy-related genes. $\# p<0.1,{ }^{*} p<0.05, * * p<0.01$, $* * * p<0.001$ compared to UL. 
Chapter 6

Table S1. Primary antibody list

\begin{tabular}{|c|c|c|c|c|}
\hline Target & Species & Supplier & Product No. & RRID \\
\hline Laminin & Mouse (histo) & Sigma & L9393 & AB_477163 \\
\hline PAX7 & Mouse (histo) & DSHB & AB528428 & AB_528428 \\
\hline AKT1 & Human & Cell signaling & 2920 & $A B \_1147620$ \\
\hline AKT1 (Ser473) & Human & Cell signaling & 4060 & AB_2315049 \\
\hline BECN1 & Human & Novus Biologicals & NB110-87318SS & AB_1236726 \\
\hline DNM1L & Human & Cell signaling & 8570 & AB_10950498 \\
\hline EIF4EBP1 & Human & Cell signaling & 9452 & AB_331692 \\
\hline EIF4IBP1 (Ser65) & Human & Cell Signaling & 9451 & AB_330947 \\
\hline FOX01 & Human & Cell signaling & 2880 & AB_2106495 \\
\hline FOXO3 & Human & Cell signaling & 2497 & AB_836876 \\
\hline FOX01 (Tyr24)/FOXO3 (Tyr32) & Human & Cell Signaling & 9464 & AB_329842 \\
\hline HK2 & Human & Cell Signaling & 2867 & AB_2232946 \\
\hline MSTN & Human & Santa Cruz & SC-6885-R & AB_640938 \\
\hline mTOR & Human & Cell signaling & 2983 & AB_2105622 \\
\hline mTOR (Ser2448) & Human & Cell Signaling & 2971 & AB_330970 \\
\hline NRF1 & Human & Abcam & AB175932 & AB_2629496 \\
\hline OXPHOS complexes & Human & MitoSciences & MS604 & AB_2629281 \\
\hline PPARGC1A & Human & Cell Signaling & 2178 & AB_823600 \\
\hline RPS6K & Human & Cell singaling & 2317 & AB_2238583 \\
\hline RPS6K (Ser235/236) & Human & Cell singaling & 4858 & AB_916156 \\
\hline TFAM & Human & Calbiochem & DR1071 & AB_10632341 \\
\hline TOMM20 & Human & Abcam & AB186734 & AB_2716623 \\
\hline BNIP3 & Human/Mouse & Cell signaling & 3769 & AB_2259284 \\
\hline BNIP3L & Human/Mouse & Cell signaling & 12396 & $A B \_2688036$ \\
\hline FUNDC1 & Human/Mouse & Santa Cruz & SC-133597 & AB_10609242 \\
\hline GABARAPL1 & Human/Mouse & Proteintech & 11010-1-AP & AB_2294415 \\
\hline MAP1LC3B & Human/Mouse & Cell signaling & 2775 & AB_915950 \\
\hline PRKN & Human/Mouse & Cell Signaling & 4211 & AB_2159920 \\
\hline SQSTM1 & Human/Mouse & Cell Signaling & 5114 & AB_10624872 \\
\hline ULK1 & Human/Mouse & Cell Signaling & 8054 & AB_11178668 \\
\hline pULK (ser556)/pULK1 (ser555) & Human/Mouse & Cell Signaling & 5869 & AB_10707365 \\
\hline pULK (ser758)/pULK1 (ser757) & Human/Mouse & Cell Signaling & 6888 & AB_10829226 \\
\hline
\end{tabular}


Table S2a: qPCR primer sequences of human genes of interest and reference genes

\begin{tabular}{|c|c|c|c|}
\hline \multicolumn{4}{|c|}{ Genes of interest (human) } \\
\hline Target & Source & Forward $\left(5^{\prime}-3^{\prime}\right)$ & Reverse (5'-3') \\
\hline ATG7 & ENSG00000197548 & GATTGCCTAGGGGGTGGTAA & TGCTCATGTTGAATCCTCTGG \\
\hline BECN1 & ENSG00000126581 & AATGCAACCTTCCACATCTGGC & CCCAGCCTGAAGTTATTGATTGTGC \\
\hline BNIP3 & ENSG00000176171 & AGCGCCCGGGATGCA & CCCGTTCCCATTATTGCTGAA \\
\hline BNIP3L & ENSG00000104765 & CTGCGAGGAAAATGAGCAGTCTCT & GCCCCCCATTTTTCCCATTG \\
\hline CALCOCO2 & ENSG00000136436 & TCTTTGCCGTATCAAGTACCTACT & GGCATTTCTTGATGGAGAGCG \\
\hline CCND1 & ENSG00000110092 & ACCGCCTCACACGCTTCCTCT & GCCTGGCGCAGGCTTGACTC \\
\hline CDH15 & ENSG00000129910 & CCTGGACATCGCCGACTTCA & TGGGGTCACTATCTGCAGCC \\
\hline CKM & ENSG00000104879 & CTGAGCAAGCACCCCAAGTT & CCACCATGAGCTTCACACCA \\
\hline COX4I1 & ENSG00000131143 & CCATGGATGAGAAAGTCGAGT & CGTTCGAGCCCCTGTTCA \\
\hline DNM1L & ENSG00000087470 & CGACTCATTAAATCATATTTTCTCATTGTCAG & TGCATTACTGCCTTTGGCACACT \\
\hline FBXO32 & ENSG00000156804 & GAAGAAACTCTGCCAGTACCACTTC & CCCTTTGTCTGACAGAATTAATCG \\
\hline FIS1 & ENSG00000214253 & CCTGGTGCGGAGCAAGTACAA & TCCTTGCTCCCTTTTGGGCAG \\
\hline FOXO1 & ENSG00000150907 & CCTGGACATGCTCAGCAGACATC & TTGGGTCAGGCGGTTCATACC \\
\hline FOXO3 & ENSG00000118689 & CACTGGTGCTAAGCAGGCCTCAT & ATGGCGTGGGATTCACAAAGGT \\
\hline FUNDC1 & ENSG00000069509 & GAAACGAGCGAACAAAGCAG & GCAAAAAGCCTCCCACAAAT \\
\hline GABARAPL1 & ENSG00000139112 & ATCGGAAAAAGGAAGGAGAAAAGATC & CAGGCACCCTGGCTTTTGG \\
\hline MAP1LC3B & ENSG00000140941 & ACCATGCCGTCGGAGAAGAC & TCTCGAATAAGTCGGACATCTTCTACTCT \\
\hline MFN1 & ENSG00000171109 & CTGAGGATGATTGTTAGCTCCACG & CAGGCGAGCAAAAGTGGTAGC \\
\hline MFN2 & ENSG00000116688 & TGGACCACCAAGGCCAAGGA & TCTCGCTGGCATGCTCCAC \\
\hline MSTN & ENSG00000138379 & AACCTTCCCAGGACCAGGAGAA & TGTCTGTTACCTTGACCTCTAAAAACGG \\
\hline MT-CO2 & ENSG00000198712 & ACCTGCGACTCCTTGACGTT & GGGGGCTTCAATCGGGAGTA \\
\hline MYF5 & ENSG00000111049 & TGCCCGAATGTAACAGTCCT & AGCAATCCAAGCTGGATAAGG \\
\hline MYH8 & ENSG00000133020 & CAGGACTTGGTAGATAAATTACAGGCGA & AGCATTGGATTGTTCCTCAGCC \\
\hline MYH9 & ENSG00000100345 & AACGCCGAGCAGTACAAGGA & GAGCTGACTTCGCGGTTCAT \\
\hline MYMK & ENSG00000187616 & CGTCACGACATCCTGGAGTA & ATGTTGACCTCTTGGGTTCG \\
\hline MYOD1 & ENSG00000129152 & CAATCCAAACCAGCGGTTGC & CCTCGATATAGCGGATGGCGT \\
\hline MYOG & ENSG00000122180 & TCAGCGCCAACCCAGG & GGTGAGGGAGTGCAGGTTGT \\
\hline NRF1 & ENSG00000106459 & GCACCTTTGGAGAATGTGGT & CTGGGATAAATGCCCGAAG \\
\hline OPA1 & ENSG00000198836 & TACCAAAGGCATTTTGTAGATTCTGAGTT & GCATGCGCTGTATACGCCAA \\
\hline OPTN & ENSG00000123240 & AAGGAGCAACTGGCATTGCA & TCTCCATCAAGGACTGCCTG \\
\hline PAX7 & ENSG00000009709 & TACAGCACCACCGGCTACAGCGTG & CTGGCCGTACTGGCCGTACTGATAG \\
\hline PCNA & ENSG00000132646 & TCTGCAAGTGGAGAACTTGGAA & GAAGTTCAGGTACCTCAGTGCAA \\
\hline PINK1 & ENSG00000158828 & GTCTCGGCCTGTCAGGAGAT & CAAGCGTCTCGTGTCCAAC \\
\hline PPARD & ENSG00000112033 & TGACCAAAAAGAAGGCCCGC & GTCGTGGATCACAAAGGGCG \\
\hline PPARGC1A & ENSG00000109819 & GACCAGTGCTACCTGAGAGAGACTT & GCTCGGCTCGGATTTCCT \\
\hline PPARGC1B & ENSG00000155846 & GGCGCTTTGAAGTGTTTGGTGA & TGATGAAGCCGTACTTCTCGCCT \\
\hline PPRC1 & ENSG00000148840 & GCCCTTTGATCTCTGCTTTGGG & AAGTCTTCCCGGTTGGAGTCAAG \\
\hline PRKN & ENSG00000185345 & GGTTTGCCTTCTGCCGGGAATG & CTTTCATCGACTCTGTAGGCCTG \\
\hline SQSTM1 & ENSG00000161011 & GGTGCACCCCAATGTGATCT & CGCAGACGCTACACAAGTCG \\
\hline TFAM & ENSG00000108064 & GAAAGATTCCAAGAAGCTAAGGGTGATT & TCCAGTTTTCCTTTACAGTCTTCAGCTTTT \\
\hline TRIM54 & ENSG00000158022 & GCGAGGTGGCCCCATT & GATGGTCTGCACACGGTCATT \\
\hline ULK1 & ENSG00000177169 & TGCCCGGTGTCCTGGTCCTC & CAGGGGCAGCAGTTGAGGCG \\
\hline \multicolumn{4}{|c|}{ Reference genes } \\
\hline RPLPO & ENSG00000089157 & TCTACAACCCTGAAGTGCTTGATATC & GCAGACAGACACTGGCAACATT \\
\hline$B 2 M$ & ENSG00000196262 & CATCTGCACTGCCAAGACTGA & TTCATGCCTTCTTTCACTTTGC \\
\hline PPIA & ENSG00000166710 & CTGTGCTCGCGCTACTCTCTCTT & TGAGTAAACCTGAATCTTTGGAGTACGC \\
\hline
\end{tabular}


Table S2b: qPCR primer sequences of mouse genes of interest and reference genes

\begin{tabular}{|c|c|c|c|}
\hline Target & Source & Forward (5'-3') & Reverse (5'-3') \\
\hline Atg7 & ENSMUSG00000030314 & TTCCTGTCAGCCTGGCATTT & GCAGGGTAAGACCGGTCAAG \\
\hline Becn1 & ENSMUSG00000035086 & CAGGATGGTGTCTCTCGAAGATT & TCTCCGGCTGAGGTTCTCC \\
\hline Bnip3l & ENSMUSG00000022051 & AGTCGGGACAGAGCAGCTCAAG & TCAAACATGATCTGCCCATCTTCTT \\
\hline Ckm & ENSMUSG00000030399 & AGGTTTTCCGCCGCTTCT & CGGTGCCCAGGTTGGA \\
\hline Fundc1 & ENSMUSG00000025040 & CGAGTATTTGGCCACAGTTCC & CCACTGTGACTGGCAACCTG \\
\hline Gabarapl1 & ENSMUSG00000030161 & CCCTCCCACCAGTGCTACCAT & TCATCACTGTAGGCCACATACAGAAAA \\
\hline Map1/c3b & ENSMUSG00000031812 & GAGCAGCACCCCACCAAGAT & CGTGGTCAGGCACCAGGAA \\
\hline Mstn & ENSMUSG00000026100 & GGCCATGATCTTGCTGTAACCT & CGGCAGCACCGGGATT \\
\hline Myh8 & ENSMUSG00000055775 & GGAAGACCGCAAGAATGTGC & TCTGCGCTGATTTTGGTGTG \\
\hline Myh9 & ENSMUSG00000022443 & AACGCGGAACAGTTCAAGGA & CGGTCTCTGTGGCATCTTCC \\
\hline Mymk & ENSMUSG00000009214 & CTGGCCGACTTTGATGAACC & TGCTCTTGTCGGGGTACAGG \\
\hline Optn & ENSMUSG00000026672 & GAGCTGCAGGTGGAGAGCAT & CCACCTTTTCTGCCTGTTGC \\
\hline Pink1 & ENSMUSG00000028756 & GTCCTGAAGGGAGCAGACG & TTAAGATGGCTTCGCTGGAG \\
\hline Prkn & ENSMUSG00000023826 & CTGGCTGTCCCAACTCCCT & CCTCGGCCCCATACTGC \\
\hline Sqstm1 & ENSMUSG00000015837 & TGTGGAACATGGAGGGAAGAGA & GCAGCTGCTTGGCTGAGTGT \\
\hline \multicolumn{4}{|c|}{ Reference genes } \\
\hline Rplpo & ENSMUSG00000067274 & GGACCCGAGAAGACCTCCTT & GCACATCACTCAGAATTTCAATGG \\
\hline Canx & ENSMUSG00000020368 & GCAGCGACCTATGATTGACAACC & GCTCCAAACCAATAGCACTGAAAGG \\
\hline Gapdh & ENSMUSG00000057666 & CAACTCACTCAAGATTGTCAGCAA & TGGCAGTGATGGCATGGA \\
\hline Ppia & ENSMUSG00000071866 & CATCTGCACTGCCAAGACTGA & TTCATGCCTTCTTTCACTTTGC \\
\hline Tuba1a & ENSMUSG00000072235 & CGTAGACCTGGAACCCACGGT & TGCCTGTGATGAGCTGCTCA \\
\hline Axin1 & ENSMUSG00000024182 & AGTGGATCATTGAGGGAGAGA & GCCCCAGGACGCTCGAT \\
\hline
\end{tabular}


Table S3: Cluster composition

\begin{tabular}{|c|c|c|c|c|c|c|}
\hline \multicolumn{2}{|c|}{ Process } & Cluster 1 & Cluster 2 & Cluster 3 & Cluster 4 & Total \\
\hline \multicolumn{7}{|c|}{ Protein turnover } \\
\hline \multirow{2}{*}{ Protein synthesis } & Count & 7 & 0 & 1 & 4 & 12 \\
\hline & $\%$ within process & $58.3 \% * *$ & $0.0 \%$ & $8.3 \%$ & $33.3 \%$ & $100.0 \%$ \\
\hline \multirow{2}{*}{$\begin{array}{l}\text { Ubiquitin proteasome } \\
\text { system }\end{array}$} & Count & 4 & 0 & 1 & 2 & 7 \\
\hline & $\%$ within process & $57.1 \%$ & $0.0 \%$ & $14.3 \%$ & $28.6 \%$ & $100.0 \%$ \\
\hline \multirow{2}{*}{ Autophagy } & Count & 9 & 0 & 4 & 6 & 19 \\
\hline & $\%$ within process & $47.4 \%$ & $0.0 \%$ & $21.1 \%$ & $31.6 \%$ & $100.0 \%$ \\
\hline \multicolumn{7}{|c|}{ Mitochondrial turnover } \\
\hline \multirow{2}{*}{$\begin{array}{l}\text { Mitochondrial } \\
\text { biogenesis }\end{array}$} & Count & 1 & 3 & 6 & 2 & 12 \\
\hline & $\%$ within process & $8.3 \%$ & $25.0 \%$ & $50.0 \%$ & $16.7 \%$ & $100.0 \%$ \\
\hline \multirow{2}{*}{ Mitophagy } & Count & 1 & 2 & 4 & 2 & 9 \\
\hline & $\%$ within process & $11.1 \%$ & $22.2 \%$ & $44.4 \%$ & $22.2 \%$ & $100.0 \%$ \\
\hline \multirow{2}{*}{ Mitochondrial mass } & Count & 0 & 0 & 0 & 6 & 6 \\
\hline & $\%$ within process & $0.0 \%$ & $0.0 \%$ & $0.0 \%$ & $100.0 \% * *$ & $100.0 \%$ \\
\hline \multirow{2}{*}{$\begin{array}{l}\text { Mitochondrial } \\
\text { dynamics }\end{array}$} & Count & 0 & 1 & 3 & 2 & 6 \\
\hline & $\%$ within process & $0.0 \%$ & $16.7 \%$ & $50.0 \%$ & $33.3 \%$ & $100.0 \%$ \\
\hline \multicolumn{7}{|c|}{ Myonuclear turnover } \\
\hline \multirow{2}{*}{ Myogenesis } & Count & 2 & 3 & 4 & 4 & 13 \\
\hline & $\%$ within process & $15.4 \%$ & $23.1 \%$ & $30.8 \%$ & $30.8 \%$ & $100.0 \%$ \\
\hline \multirow{2}{*}{$\begin{array}{l}\text { Muscle mass and } \\
\text { function }\end{array}$} & Count & 1 & 4 & 2 & 2 & 9 \\
\hline & $\%$ within process & $11.1 \%$ & $44.4 \%$ & $22.2 \%$ & $22.2 \%$ & $100.0 \%$ \\
\hline \multirow{2}{*}{ Total } & Count & 25 & 13 & 25 & 30 & 93 \\
\hline & $\%$ within process & $26.9 \%$ & $14.0 \%$ & $26.9 \%$ & $32.3 \%$ & $100.0 \%$ \\
\hline
\end{tabular}

Distribution of markers and mediators associated with the indicated processes, presented as the number (counts) or percentage (\% within process) of markers per cluster. ${ }^{* *} p<0.01$ Pearson's chi-square post-hoc based on adjusted residuals, with Bonferroni correction. 
Iron deficiency-induced loss of skeletal muscle mitochondria; the role of mitophagy and secretion of mitochondriacontaining vesicles

P.A. Leermakers, A.H.V. Remels, M.I. Zonneveld, K.M. Rouschop, A.M.W.J. Schols, H.R. Gosker

Submitted 


\section{Abstract}

Background: Iron homeostasis is essential for proper mitochondrial function. Iron deficiency has been associated with skeletal muscle weakness and a decreased exercise capacity in patients with chronic disorders including heart failure and chronic obstructive pulmonary disease. We hypothesised that iron deficiency-induced loss of skeletal muscle mitochondrial content is caused by increased mitochondrial clearance.

Methods: Differentiated murine $\mathrm{C} 2 \mathrm{C} 12$ skeletal muscle cells (myotubes) were subjected to the iron chelator deferiprone. Subsequently, mitochondrial content and function, as well as abundance of key constituents of mitophagy pathways were studied in the presence or absence of pharmacological inhibition of general autophagy or knockdown of specific mitophagy-related proteins. Furthermore, we explored if mitochondria were present in extracellular vesicles (EV) in response to iron chelation.

Results: Iron chelation in myotubes resulted in an increase in BCL2/Adenovirus E1B $19 \mathrm{kDa}$ protein-interacting protein 3 (BNIP3) and BNIP3-like (BNIP3L) gene- and protein levels, and the appearance of mitochondria encapsulated by lysosome-like vesicular structures. In addition, we found that mitochondria were secreted via EV. These changes were associated with decreased cellular mitochondrial function and content. The development of these mitochondrial impairments was unaltered by autophagy inhibition, knockdown of specific mitophagy-related proteins BNIP3 and BNIP3L, or knockdown of their upstream regulator hypoxia-inducible factor 1 alpha (HIF1A).

Conclusion: Mitophagy is not essential for development of iron deficencyinduced reductions in mitochondrial content or function. The secretion of mitochondria-containing EV could represent an additional pathway via which mitochondrial content can be reduced in iron chelation-exposed myotubes. 


\section{Introduction}

Mitochondria are highly dynamic, cytoplasmic organelles that play a key role in a variety of cellular functions, of which the oxidative phosphorylationmediated synthesis of ATP from ADP is the best known. Due to its high energy requirements, skeletal muscle is a metabolically active and mitochondriarich tissue. Illustrative of the plasticity of the mitochondrial network in skeletal muscle, the number of mitochondria can vary greatly depending on physiological requirements (e.g. during physical activity or inactivity) and resource availability (e.g. during starvation) (1). Iron homeostasis is essential for proper mitochondrial function as iron is an essential component for several of the protein complexes that make up the electron transport chain, and hence is essential for cellular respiration and subsequent ATP production. Indeed, it is well established that iron deficiency in vivo results in decreased skeletal muscle mitochondrial quantity and function culminating in impaired oxidative substrate metabolism (2-7). Illustrative of its clinical significance, iron deficiency has been associated with muscle weakness and decreased exercise capacity in patients with congestive heart failure and chronic obstructive pulmonary disease $(8,9)$. However, the intra-cellular mechanisms of how iron deficiency drives this loss of skeletal muscle mitochondrial quantity and quality remain to be elucidated.

Mitochondrial clearance can be achieved via mitochondrial breakdown (1) or via secretion of mitochondria in the extracellular environment (10-12). There are several routes of mitochondrial breakdown, including mitophagy (i.e. mitochondrial autophagy), mitochondrial-derived vesicles and proteasome-mediated mitochondrial protein degradation $(1,13)$. Mitophagy has the largest breakdown capacity, as it is able to clear complete mitochondria, and it is best described in literature. During mitophagy, mitochondria are engulfed by an autophagosomal membrane which subsequently fuses with a lysosomal structure in which the mitochondria are degraded. Mitophagy can roughly be divided into receptor-mediated and ubiquitin-mediated mitophagy. Receptor-mediated mitophagy is initiated by activation of mitophagy receptors like BCL2/Adenovirus E1B $19 \mathrm{kDa}$ protein-interacting protein 3 (BNIP3), BNIP3-like (BNIP3L), and FUN14 domain-containing protein 1 (FUNDC1) (14-16), while ubiquitin-mediated mitophagy is initiated by stabilization of PTEN-induced putative kinase 1 (PINK1) and mitochondrial recruitment of Parkin (PARK2) (17-19). Although iron deficiency has been shown to result in increased mitophagy in several models including C. elegans, yeast, osteosarcoma, and neuroblastoma cells, evidence of iron deficiency-induced mitophagy in skeletal muscle is still lacking (20-22). Recently, the secretion of mitochondria via extracellular vesicles (EV) has been described 
as an alternative mechanism for mitochondrial clearance $(10,11)$. However, there is currently no data reporting secretion of mitochondria in $\mathrm{EV}$ from skeletal muscle, nor in relation to alterations in iron metabolism.

In this study, we hypothesised that loss of skeletal muscle mitochondrial content and function induced by iron deficiency is caused by increased mitochondrial clearance. Differentiated murine skeletal muscle cells were subjected to an iron chelating compound. Subsequently, parameters related to mitochondrial content and function, as well as abundance of key constituents of mitophagy pathways were studied in the presence or absence of inhibition of general autophagy or specific knockdown of several important mitophagy-related proteins. Furthermore, we explored if mitochondria were packaged into extracellular vesicles in response to iron chelation.

\section{Materials \& Methods}

\section{Cell culture}

Murine skeletal muscle cells (C2C12) were obtained from the American Type Culture Collection (CRL1772, Manassas, VA, USA). Cells were cultured $\left(37^{\circ} \mathrm{C}\right.$, $5 \%$ CO2), and seeded in matrigel-coated dishes (\#354234, BD Biosciences, Breda, the Netherlands) at $85 \%$ confluence, in low glucose $(1 \mathrm{~g} / \mathrm{L}$ ) Dulbecco's Modified Eagle Medium (DMEM; \#22320-022) containing $50 \mathrm{U} / \mathrm{ml}$ penicillin and $50 \mu \mathrm{g} / \mathrm{ml}$ streptomycin (\#15140-122) and 9\% fetal bovine serum (all from Gibco, Bleiswijk, the Netherlands). Cells were subsequently differentiated for 5 days in high glucose DMEM (4.5 g/L) (\#41966-029) containing $50 \mathrm{U} / \mathrm{ml}$ penicillin and $50 \mu \mathrm{g} / \mathrm{ml}$ streptomycin (\#15140-122, all from Gibco) and 1\% heat-inactivated fetal bovine serum (differentiation medium: DM). For indicated experiments, a C2C12 cell line stably expressing a hypoxia-inducible factor (HIF) reporter plasmid, which expresses the luciferase gene controlled by a promoter bearing five consecutive HIF-responsive elements (HREs) from the human vascular endothelial growth factor A (VEGFA) promoter (5HRE/hCMVmp-luc), was used. Subsequently, cells were exposed to $300 \mu \mathrm{M}$ DFP (Deferiprone, \#379409, Sigma-Aldrich, Zwijndrecht, the Netherlands) in presence or absence of 100 MM Fe(III)Cl3, 5 mM 3-MA (3-methyladenine, \#s2762, Bioconnect, Huissen, the Netherlands), or $10 \mathrm{nM}$ BAFA1 (Bafilomycin A1, \#B1793, Sigma-Aldrich).

For indicated experiments, transfection of $2 \mathrm{nM}$ of target-mRNA-specific silencer select siRNA (BNIP3 (\#s63059), BNIP3L (\#s63062), GABARAPL1 (\#s81311)) or silencer select scrambled control siRNA (\#4390843) (all from Thermo Scientific, Landsmeer, the Netherlands) was initiated in presence of 
0.12\% RNAiMAX (\#13778150, Thermo Scientific). Similarly, transfection of $5 \mathrm{nM}$ of HIF1A-specific stealth siRNA (\#MSS205126) or low GC scrambled control siRNA (\#12935200) (Thermo Scientific) was initiated in presence of $0.2 \%$ RNAiMAX. All transfections were performed in DM $24 \mathrm{~h}$ prior to DFP exposure.

\section{Isolation of extracellular vesicles}

Cells were cultured for $24 \mathrm{~h}$ in EV-depleted DM, generated by overnight centrifugation of DM in polyallomer tubes (Beckman Coulter, Woerden, the Netherlands) at $100,000 \times \mathrm{g}$ in a SW32 rotor in a XPN-90 Ultracentrifuge (Beckman Coulter) at $4^{\circ} \mathrm{C}$. Conditioned medium was collected from $150 \mathrm{~cm} 2$ differentiated myotubes, centrifuged at $200 \times \mathrm{g}$ for $10 \mathrm{~min}$ at $4{ }^{\circ} \mathrm{C}$, and transferred to a new tube and centrifuged at $2,000 \times \mathrm{g}$ for $10 \mathrm{~min}$ at $4{ }^{\circ} \mathrm{C}$ to discard dead cells and debris. Medium was transferred to polyallomer tubes and centrifuged at $16,000 \times \mathrm{g}$ for $30 \mathrm{~min}$ at $4{ }^{\circ} \mathrm{C}$ and subsequently at $100,000 \times \mathrm{g}$ for $65 \mathrm{~min}$ at $4{ }^{\circ} \mathrm{C}$. The pellets from the $16,000 \times \mathrm{g}$ and $100,000 \times \mathrm{g}$ centrifugation steps were resuspended in Phosphate Buffered Saline (PBS) and overlayed by a $2.5-0.4 \mathrm{M}$ sucrose gradient in polyallomer tubes and centrifuged overnight at $188,000 \mathrm{xg}$ in an SW41 rotor at $4{ }^{\circ} \mathrm{C}(23,24)$. EV-containing sucrose fractions were collected from both gradients based on EV density characteristics $(1.12-1.19 \mathrm{~g} / \mathrm{ml})$, and were subsequently diluted in PBS and centrifuged at $100,000 \mathrm{xg}$ in an SW32 rotor for $65 \mathrm{~min}$ at $4{ }^{\circ} \mathrm{C}$. Pellets were dissolved in $30 \mu \mathrm{IBS}$ and were used for subsequent analyses.

\section{RNA extraction and qPCR}

Cells were lysed in $350 \mu \mathrm{l}$ RLT lysis buffer containing $1 \% \beta$-mercaptoethanol by running the sample through a 20 gauge needle. RNA was extracted using the RNeasy plus mini kit (\#74134, Qiagen, Venlo, the Netherlands) according to the manufacturer's protocol. 400 ng of RNA was used for cDNA synthesis using the tetro cDNA synthesis kit (\#BIO-65043, Bioline, Alphen aan de Rijn, the Netherlands) according to manufacturer's protocol.

$4.4 \mu \mathrm{l}$ of $1 / 50$ diluted cDNA was used for quantitative PCR amplification using target- and species-specific primers (Table 1 ) and $2 \mathrm{X}$ Sensimix $^{\mathrm{TM}}$ SYBRC \& Fluorescein mix (\#QT615-20, Bioline) on a LightCycler480 384-wells PCR machine (Roche, Almere, the Netherlands). Specificity of PCR amplification was tested with melt curve analysis, and expression levels of genes of interest were corrected using a normalization factor calculated based on the expression of 4 different housekeepers (B2m, Ppia, RplpO, and Rp/13A) using the GeNorm software (Primerdesign, Southampton, USA). 
Table 1: Primers used for qPCR

\begin{tabular}{|c|c|c|c|}
\hline & Sense primer & Antisense primer & Ensembl identifier \\
\hline$B 2 m$ & СTTTCTGGTGCTTGTCTCACTGA & GTATGTTCGGCTTCCCATTCTC & ENSMUSG00000060802 \\
\hline Ppia & TTCCTCCTTTCACAGAATTATTCCA & CCGCCAGTGCCATTATGG & ENSMUSG00000071866 \\
\hline Rp/13A & САCTCTGGAGGAGAAACGGAAGG & GCAGGCATGAGGCAAACAGTC & ENSMUSG00000074129 \\
\hline Rplpo & GGACCCGAGAAGACCTCCTT & GCACATCACTCAGAATTTCAATGG & ENSMUSG00000067274 \\
\hline Bnip3 & AGGTTTTCCTTCCATCTCTGTTACTG & TGTGTGAACAGAAGTCAGATCCAAA & ENSMUSG00000078566 \\
\hline Bnip3l & AGTCGGGACAGAGCAGCTCAAG & TCAAACATGATCTGCCCATCTTCTT & ENSMUSG00000022051 \\
\hline Fundc1 & CGAGTATTTGGCCACAGTTCC & CCACTGTGACTGGCAACCTG & ENSMUSG00000025040 \\
\hline Pink1 & GTCCTGAAGGGAGCAGACG & TTAAGATGGCTTCGCTGGAG & ENSMUSG00000028756 \\
\hline Park2 & CTGGCTGTCCCAACTCCCT & CCTCGGCCCCATACTGC & ENSMUSG00000023826 \\
\hline$m t-\operatorname{Cox} 2$ & CCATCCCAGGCCGACTAA & ATTTCAGAGCATTGGCCATAGAA & ENSMUSG00000064354 \\
\hline Hif1a & AATGAAGTGCACCCTAACAAGCCG & TGGCCCGTGCAGTGAAGC & ENSMUSG00000021109 \\
\hline
\end{tabular}

\section{Mitochondrial DNA copy number}

Cells were lysed in $350 \mu$ l DNA lysis buffer $(0.1 \mathrm{M}$ Tris/ $\mathrm{HCl}$ pH 8.5, $5 \mathrm{mM}$ EDTA $\mathrm{pH} 8.0,0.2 \%$ SDS and $0.2 \mathrm{M} \mathrm{NaCl}$ ) or $5 \mu \mathrm{l}$ of EV in PBS was added to $350 \mu \mathrm{LNA}$ lysis buffer. Proteinase $\mathrm{K}$ was added to a final concentration of $0.2 \mathrm{mg} / \mathrm{ml}$ and the lysates were incubated overnight at $55^{\circ} \mathrm{C}$. Lysates were centrifuged at 20,000 x g for $5 \mathrm{~min}$ and DNA from the supernatants was precipitated with isopropanol. DNA pellets were washed twice with $70 \%$ ethanol and air-dried. DNA pellets were dissolved in elution buffer $(10 \mathrm{mM}$ Tris/ $\mathrm{HCl}, \mathrm{pH} 8.0)$ and heated to $55^{\circ} \mathrm{C}$ for $2 \mathrm{~h}$ followed by overnight incubation at $4{ }^{\circ} \mathrm{C}$. For DNA isolated from cells, $4.4 \mu \mathrm{l}$ 1/1000 diluted DNA was used for qPCR as described above using mitochondrial DNA (mtDNA) specific mt-Cox2 or genomic DNA (gDNA)-specific Ppia primers (table 1 ) and the mtDNA/gDNA ratio was calculated. For DNA isolated from EV, $4.4 \mu \mathrm{l}$ 1/20 diluted DNA was used for qPCR as described above, using mtDNA-specific mt-Cox2 primers (table 1 ).

\section{Protein extraction and Western Blotting}

Cells were lysed in $100 \mu \mathrm{l}$ whole cell lysis buffer $(150 \mathrm{mM} \mathrm{NaCl}, 1 \%$ Nonidet, 1mM DTT, 1x cOmplete protease inhibitor cocktail (\#11697498001, Sigma-Aldrich), $20 \mathrm{mM}$ tris, $\mathrm{pH}$ 7.4). Lysates were incubated while rotating for $30 \mathrm{~min}$ at $4{ }^{\circ} \mathrm{C}$ and subsequently centrifuged at $20,000 \times \mathrm{g}$ for $30 \mathrm{~min}$ at $4{ }^{\circ} \mathrm{C}$. Protein concentration was determined using the Pierce ${ }^{\mathrm{TM}}$ BCA Protein Assay Kit (\#23225, Thermo Scientific) according to the manufacturer's protocol. Lysates (1 $\mu \mathrm{g} / \mu \mathrm{l})$ were aliquoted in sample buffer $(0.25 \mathrm{M}$ Tris- $\mathrm{HCl}, 8 \%(\mathrm{w} / \mathrm{v}) \mathrm{SDS}$, $40 \%$ (v/v) glycerol, 0.4M DTT, 0.04\% (w/v) Bromophenol Blue, $\mathrm{pH} 6.8$ ) and boiled for $5 \mathrm{~min}$ at $95{ }^{\circ} \mathrm{C}$. $10 \mu \mathrm{g}$ of protein per sample was run through a Criterion 26-wells 12\% precast gel (\#3450119, Bio-Rad Laboratories B.V., Veenendaal, the Netherlands) in 1x MES buffer (\#1610796, Bio-Rad Laboratories B.V.) at 100 volts and was subsequently blotted on a nitrocellulose membrane by electroblotting. At least two protein ladders were loaded on each gel (\#1610373, Precision Plus 
Protein $^{\mathrm{TM}}$ All Blue Standards, Bio-Rad Laboratories B.V.). $20 \mu \mathrm{l}$ EV in PBS was combined with $20 \mu \mathrm{l} 2 \mathrm{x}$ sample buffer and boiled for $5 \mathrm{~min}$ at $95^{\circ} \mathrm{C}$. $20 \mu \mathrm{l} \mathrm{EV}$ in sample buffer was run through Criterion gels as described above.

Membranes were incubated in Ponceau S (\#P7170-1L, Sigma-Aldrich) for 5 min and washed with milliQ before they were imaged using the LAS-3000 (Fujifilm Life Sciences B.V., Tilburg, the Netherlands) or the Amersham ${ }^{\text {TM }}$ Imager 600 (GE Healthcare Life Sciences, Eindhoven, the Netherlands). Total protein Ponceau $\mathrm{S}$ quantification was used as correction for gel-loading. Subsequently, membranes were washed, blocked with 3\% non-fat, dried milk (Campina, Amersfoort, the Netherlands) in TBS-Tween-20 (0.05\%) for $1 \mathrm{~h}$, washed, and incubated overnight at $4{ }^{\circ} \mathrm{C}$ with different protein-specific primary antibodies against: BNIP3 (\#3769S, RRID:AB_2259284, Cell Signalling Technology), BNIP3L (\#12396, RRID:AB_2688036, Cell Signalling Technology), FUNDC1 (\#sc-133597, RRID:AB_10609242, Santa Cruz Biotechnology), microtubule-associated protein light chain 3 (LC3)B (\#2775, RRID:AB_915950, Cell Signalling Technology), Heatshock protein (HSP)70 (\#NB110-96425, RRID:AB_1262360, Novus), Flotillin-1 (FLOT1) (\#610821, RRID:AB_398140, BD Biosciences), and oxidative phosphorylation (OXPHOS) complex subunits (\#MS604, RRID:AB_2629281, MitoScience LLC) all diluted in $3 \%$ non-fat, dried milk or $5 \%$ bovine serum albumin in TBS-Tween-20. Membranes were washed and incubated with Horseradish peroxidase-labelled, primary antibody-specific, secondary antibody (\#BA-9200, \#BA-1000, Vector Laboratories, Amsterdam, the Netherlands) (1:10,000 diluted in 3\% non-fat, dried milk in TBS-Tween-20) for $1 \mathrm{~h}$ at room temperature. Membranes were washed and incubated with either $0.5 \mathrm{x}$ SuperSignal West Pico Chemiluminescent Substrate or $0.25 x$ SuperSignal West Femto Chemiluminescent Substrate (\#34578,\#34095, Thermo Scientific) for 5 min, depending on the expected signal strength. Photographs were taken with the LAS-3000 or Amersham ${ }^{\mathrm{TM}}$ Imager 600 and analysed with ImageQuant TL software (GE Healthcare Life Sciences).

\section{Enzyme activity assays}

Cells were lysed in $100 \mu \mathrm{l}$ SET buffer (250 mM sucrose, 2 mM EDTA, $10 \mathrm{mM}$ Tris, $\mathrm{pH}$ 7.4) and snap-frozen in liquid nitrogen. Subsequently, samples were thawed, incubated on ice for $30 \mathrm{~min}$, and centrifuged at 20,000 x g for $10 \mathrm{~min}$ at $4{ }^{\circ} \mathrm{C}$. A final concentration of $1 \%$ BSA was added to the $60 \mu$ of sample. Protein concentration was determined by the Pierce ${ }^{\mathrm{TM}}$ BCA Protein Assay Kit in the remaining supernatant. Enzyme activities were measured spectrophotometrically (Multiskan Spectrum, Thermo Lab Systems, Landsmeer, the Netherlands) as described previously for both citrate synthase (CS) and $\beta$-hydroxyacyl-CoA dehydrogenase (HADH) (25). 


\section{Respirometry}

A Seahorse XF96 sensor cartridge with Seahorse calibrant $(\mathrm{pH}=7.4)$ was incubated in a CO2-free incubator at $37{ }^{\circ} \mathrm{C} 24 \mathrm{~h}$ prior to the measurement (\#101085-004, Agilent, Santa Clara, USA). Cells were washed twice with XF assay media (DMEM with $1 x$ Glutamax, $1 \mathrm{mM}$ sodium pyruvate, $0.9 \mathrm{~g} / \mathrm{L} \mathrm{NaCl}$, $4.5 \mathrm{~g} / \mathrm{L}$ glucose, phenol red, $\mathrm{pH}=7.35 \pm 0.05$ ) and incubated for $1 \mathrm{~h}$ in assay media in a $\mathrm{CO} 2$-free incubator at $37^{\circ} \mathrm{C}$. A mitochondrial stress test was performed using final concentrations of $1 \mu \mathrm{M}$ oligomycin, $2 \mu \mathrm{M}$ carbonyl cyanide-4-(trifluoromethoxy)phenylhydrazone (FCCP) and $1 \mu \mathrm{M}$ rotenone/antimycin-A. Cells were lysed in $0.05 \%$ SDS in Hanks' Balanced Salt solution (HBSS), and protein concentration was determined with the Pierce ${ }^{\mathrm{TM}}$ BCA Protein Assay Kit to correct respirometry data.

\section{Luciferase activity}

Cells were lysed in 1x Reporter Lysis Buffer (\#E397A, Promega, Madison, USA) and snap frozen in liquid nitrogen. After thawing the lysate was centrifuged at $20,000 \times \mathrm{g}$ for $2 \mathrm{~min}$ and the soluble fraction was used for determination of luciferase activity. Samples were analysed with a single-tube luminometer with injector (\#LB9507, Berthold Technologies, Bad Wildbad, Germany) by measurement of relative luciferase units (RLU) after addition of Luciferase Assay Reagent (20 mM Tricine, $1.07 \mathrm{mM}$ [(MgCO3)4Mg(OH)2.5H2O], $2.67 \mathrm{mM} \mathrm{MgSO}$, $0.1 \mathrm{mM}$ EDTA, $33.3 \mathrm{mM}$ DTT, $270 \mu \mathrm{M}$ coenzyme $\mathrm{A}, 470 \mu \mathrm{M}$ beetle luciferin potassium salt and $580 \mu \mathrm{M}$ ATP, $\mathrm{pH}$ 7.8) to the sample. Protein concentration was determined with the Pierce ${ }^{\mathrm{TM}}$ BCA Protein Assay Kit, which was used to correct luciferase data.

\section{Electron microscopy}

Cells were fixed with $2.5 \%$ glutaraldehyde in $0.1 \mathrm{M}$ cacodylate buffer in the cell culture dish and kept in the plate until the polymerisation was completed. The cells were kept in the fixative during $24 \mathrm{~h}$ at $4{ }^{\circ} \mathrm{C}$. Subsequently, cells were washed with $0.1 \mathrm{M}$ cacodylate buffer and post-fixed with $1 \%$ osmium tetroxide in the same buffer containing $1.5 \%$ potassium ferricyanide during $1 \mathrm{~h}$ in darkness at $4{ }^{\circ} \mathrm{C}$. Then the samples were dehydrated in ethanol, infiltrated with Epon resin during 2 days, embedded in the same resin and polymerised at $60{ }^{\circ} \mathrm{C}$ during $48 \mathrm{~h}$. Ultrathin sections were obtained using a Leica Ultracut UCT ultramicrotome and mounting on Formvar-coated copper grids. They were stained with $2 \%$ uranyl acetate in water and lead citrate. Sections were observed in a Tecnai T12 electron microscope equipped with an Eagle 4kx4k CCD camera (Thermo Fisher Scientific). 
Ultrastructural features resembling a second membrane encapsulating mitochondria (indicative of autophagosomal-vesicle formation) or structures resembling lysosomes containing mitochondria (indicative of lysosomal breakdown) were identified. EV were defined as separate membrane-enclosed structures near the plasma membrane of approximately 100-1000 nm in diameter. Quantification was performed by selecting 10 random cells and counting EV across the length of approximately $50 \mu \mathrm{m}$ of plasma membrane and presenting the average number of vesicles per $10 \mu \mathrm{m}$ plasma membrane. This quantification was performed in three independent samples.

\section{Statistics}

Data were analysed using an unpaired student's t-test in case of one comparison of two datasets, or one-way ANOVA with Bonferroni as a post-hoc test in case of multiple comparisons in multiple datasets, all executed with the GraphPad Prism 5.0 software. Data are expressed as mean \pm SEM. Depicted representative western blots had equally applied brightness and contrast adjustments. All western blot quantifications were performed on original and unadjusted photographs. 


\section{Results}

Iron chelation results in impaired mitochondrial function and decreased mitochondrial quantity.

Myotubes subjected to iron chelation, i.e. exposure to $300 \mu \mathrm{M}$ DFP, had a lower maximal oxygen consumption rate (OCR), mtDNA copy number, and protein levels of the OXPHOS complex subunits NDUFB8 (complex I) and SDHB (complex II). No decreases in HADH activity, CS activity or protein levels of the OXPHOS complexes CQCRC2 (complex III) and ATP5A (complex V) were observed (Fig 1A-E).

A

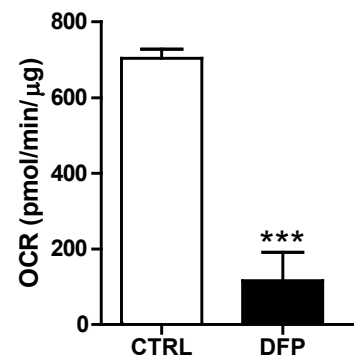

D

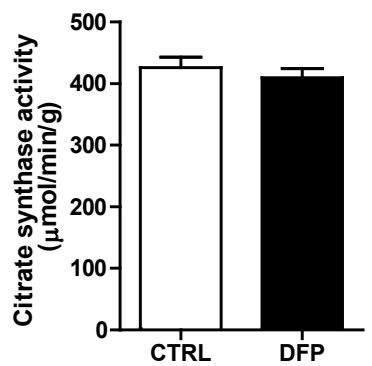

B

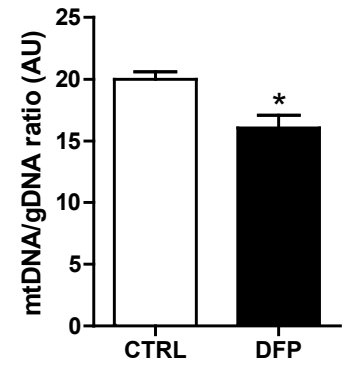

E

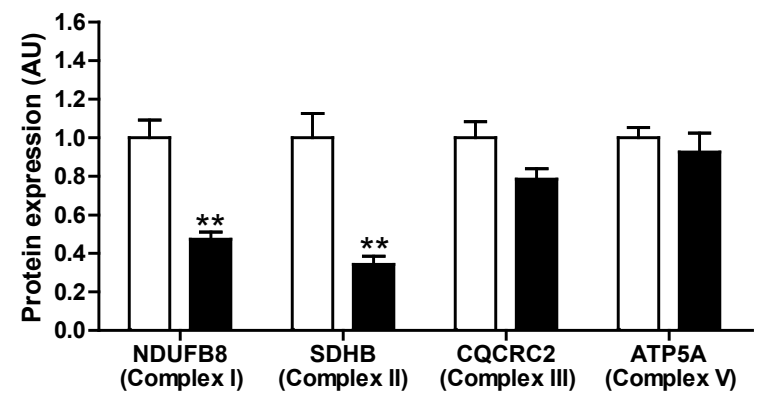

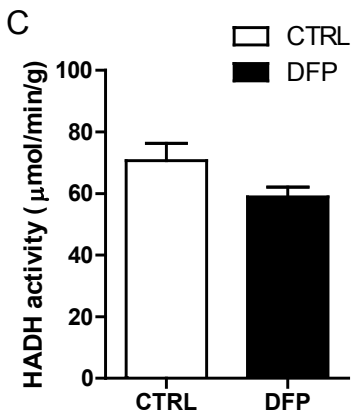

TRL

PonceauS 
To demonstrate that this decreased mitochondrial quantity resulted specifically from the iron chelation and were not mediated by any other non-specific DFP-related effect, cells were exposed to the pre-saturated iron chelator (300 $\mu \mathrm{M}$ DFP $+100 \mu \mathrm{M} \mathrm{FeCl3)}$. Exposure to pre-saturated DFP resulted in a rescue of the DFP-induced reductions in mitochondrial content (Fig $2 A, B$ ). These experiments indicate that the DFP-induced reductions in mitochondrial content did indeed result specifically from myotube iron chelation.

Iron chelation results in mitochondria engulfed in double layered membranes, mitochondrial-lysosomal co-localisation, and increased BNIP3 and BNIP3Llevels Mitochondria surrounded by double-layered membranes and mitochondria in lysosomal-like structures were observed in myotubes subjected to iron chelation, while these were not detected in untreated cells (Fig 3A). In line with this, mRNA expression of mitophagy receptors BNIP3 and BNIP3L was higher in myotubes exposed to iron chelation compared to control myotubes (Fig 3B). However, PINK1, PARK2, and FUNDC1 mRNA expression was not significantly

A

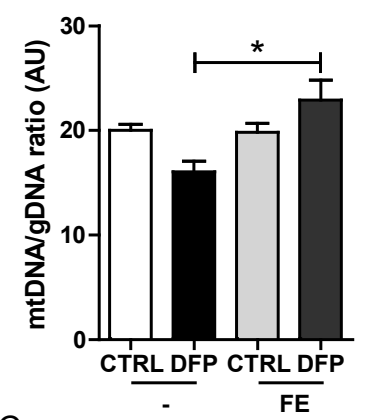

C

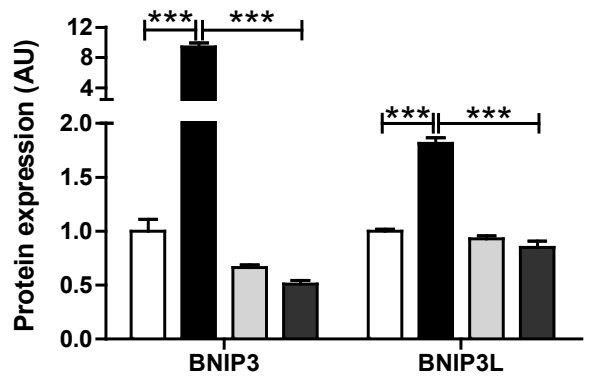

B

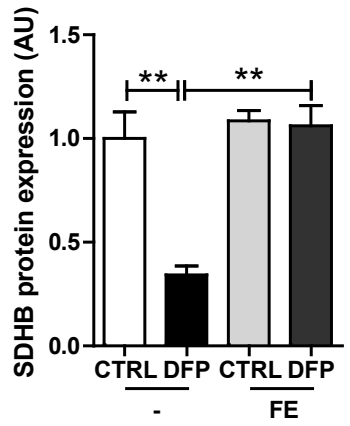

Figure 2: Pre-saturation of DFP with iron attenuates DFP-induced changes. mtDNA/gDNA ratio (A), SDHB (B), and BNIP3 and BNIP3L (C) protein levels are depicted of myotubes exposed for 24h to control (white bars), $300 \mu \mathrm{M}$ DFP (black bars), control + $100 \mu \mathrm{M}$ FE (light grey bars), or $300 \mu \mathrm{M}$ DFP + $100 \mu \mathrm{M} F E$ (dark grey bars). Data are depicted as mean + SEM $(n=3)$, and significant differences are depicted $* p<0.05, * * p<0.01, * * * p<0.001$. 
A
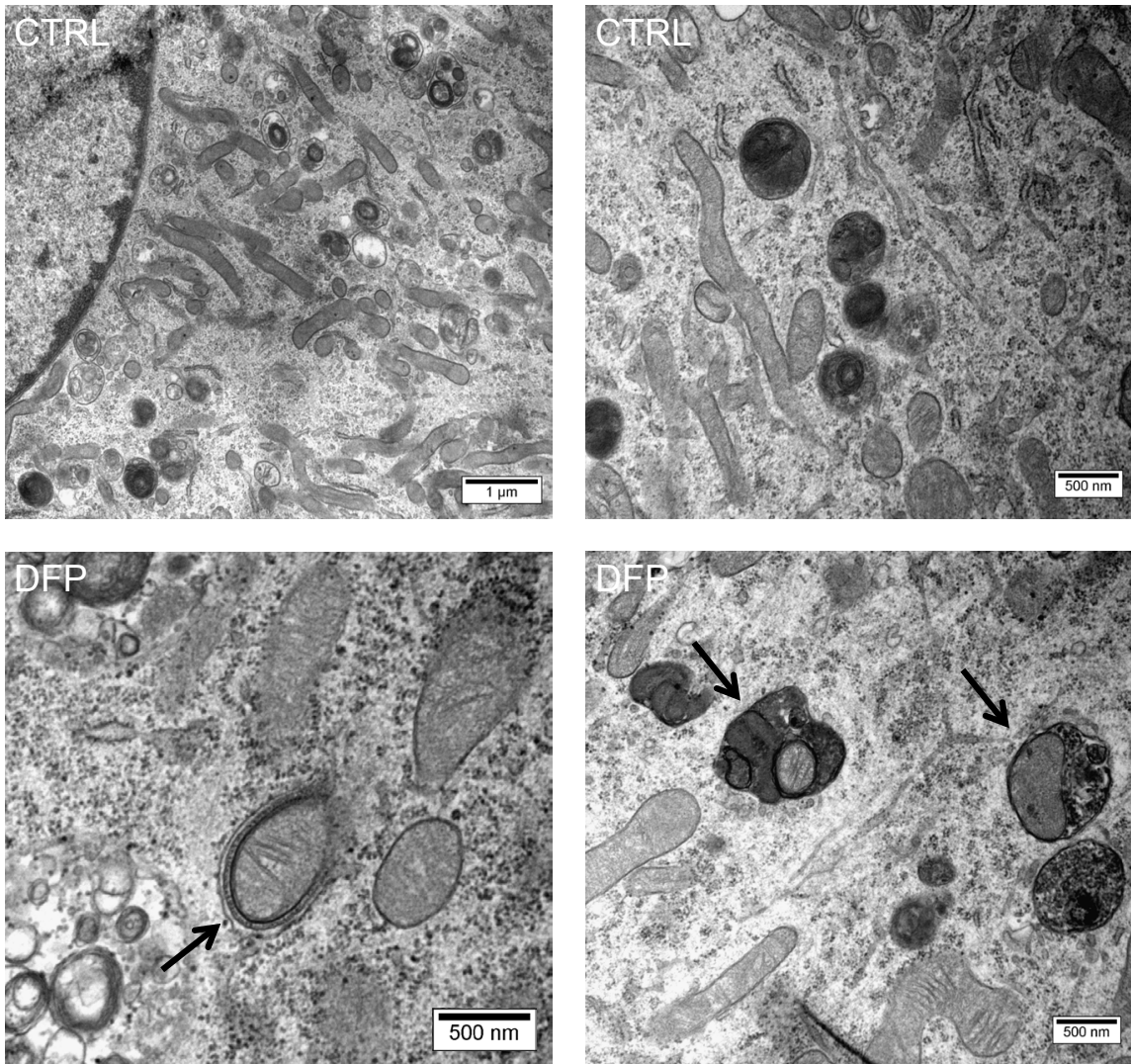

B

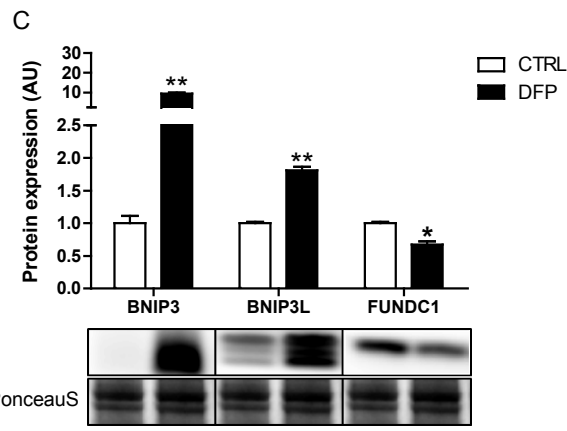

Figure 3: Iron chelation in myotubes induces mitophagy. Presence of mitophagy-events, i.e. autophagosomal-enclosed mitochondria or lysosomal-enclosed mitochondria (black arrows) in myotubes exposed for $24 \mathrm{~h}$ to control or $300 \mu \mathrm{M}$ DFP (A). mRNA expression of mitophagy receptors Bnip3, Bnip3l, and Fundc1, and ubiquitin-mediated mitophagy effectors Pink1, and Park2 is depicted of myotubes exposed for $24 \mathrm{~h}$ to control (white bars) or $300 \mu \mathrm{M}$ DFP (black bars) (B). Protein expression of mitophagy receptors BNIP3, BNIP3L and FUNDC1 is depicted of myotubes exposed to control (white bars) or $24 \mathrm{~h}$ of $300 \mu \mathrm{M}$ DFP (black bars) (C). Data are depicted as mean $+\operatorname{SEM}(\mathrm{n}=3)$, and significant differences are depicted $* p<0.05,{ }^{* *} p<0.01$. 
different in exposed myotubes (Fig 3B). Moreover, both BNIP3 and BNIP3L protein levels were higher in myotubes exposed to iron chelation (Fig 3C, Fig 2C), while FUNDC1 protein levels were lower (Fig $3 \mathrm{C}$ ). Together, these data suggest that mitophagy is increased in response to iron chelation.

A

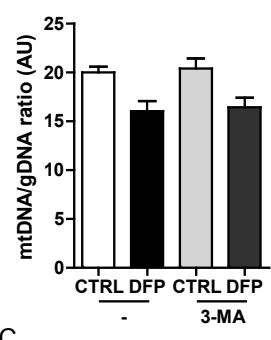

C

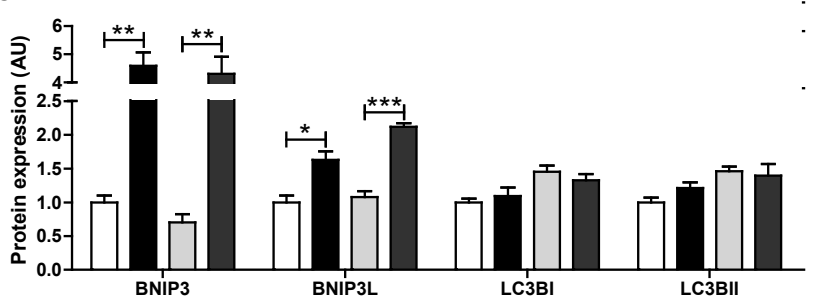

D

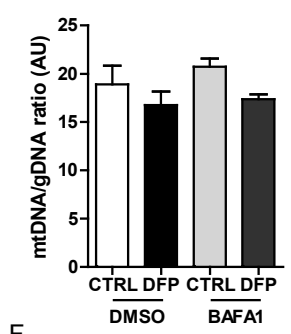

$\mathrm{F}$

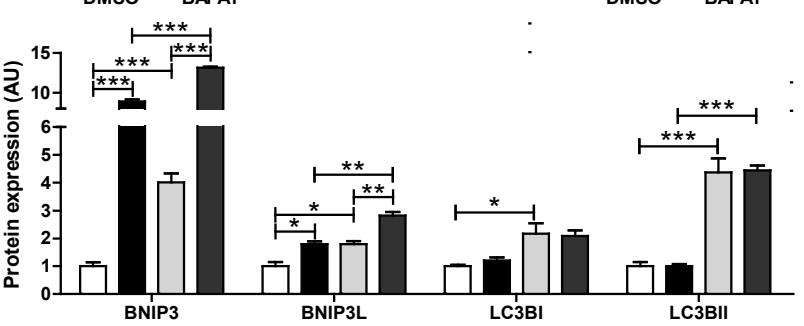

B

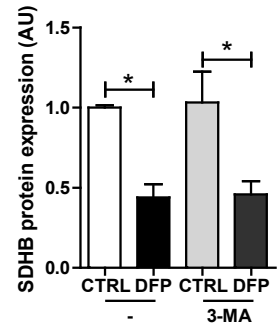

$\mathrm{E}$
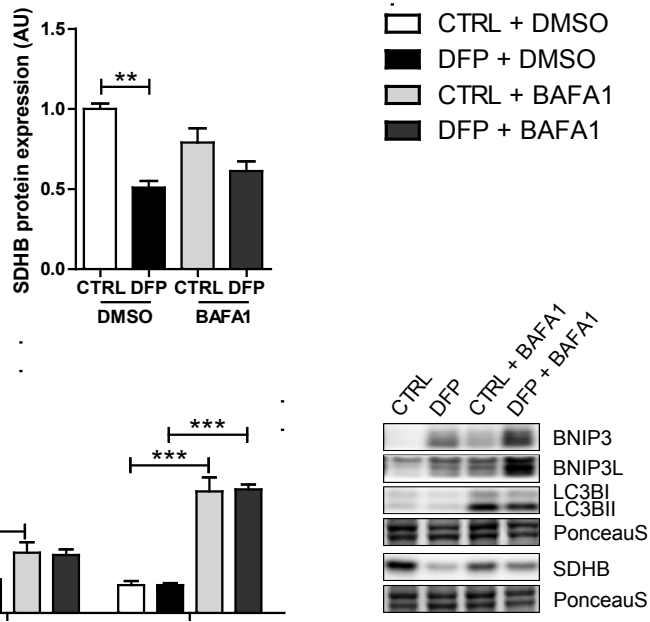

Figure 4: Inhibition of general autophagy initiation or lysosomal breakdown during iron chelation does not rescue loss of mitochondrial content. mtDNA/gDNA ratio (A), SDHB protein levels (B), BNIP3, BNIP3L, LC3BI, and LC3BII protein levels (C), are depicted of myotubes exposed for $24 \mathrm{~h}$ to control (white bars), $300 \mu \mathrm{M}$ DFP (black bars), control + 3-MA (light grey bars), or $300 \mu \mathrm{M}$ DFP + 3-MA (dark grey bars). mtDNA/gDNA ratio (D), SDHB protein levels (E), BNIP3, BNIP3L, LC3BI, and LC3BII protein levels (F), are depicted of myotubes exposed for $24 \mathrm{~h}$ to control (white bars),

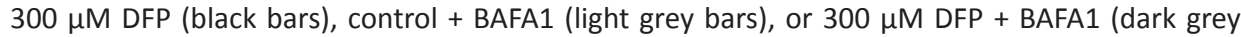
bars). Data are depicted as mean + SEM $(n=3)$, and significant differences are depicted compared with control or indicated bars $* p<0.05, * * p<0.01, * * * p<0.001$. 
Iron chelation-induced decrease in mitochondrial content is not autophagy-dependent

To test if the iron chelation-mediated decrease in mitochondrial content was autophagy-dependent, autophagy initiation and lysosomal breakdown were inhibited using the type III Phosphatidylinositol 3-kinases (PI-3K) inhibitor 3-MA and a vacuolar type $\mathrm{H}^{+}$-ATPase inhibitor BAFA1 respectively. DFP-induced decreases in mitochondrial content were not influenced by the presence of either 3-MA or BAFA1 (Fig 4A, B, D, E). Accumulation of BNIP3, BNIP3L and LC3B was not observed during inhibition of autophagy initiation with 3-MA (Fig 4C), while protein levels of BNIP3, BNIP3L and LC3B were accumulating after inhibition of lysosomal breakdown with BAFA1 (Fig 4F). Since SDHB showed the largest decrease after DFP exposure (Fig 1), SDHB protein levels were reported as a marker of the DFP-induced decreased mitochondrial content. Together, these data indicate that the iron chelation-induced decrease in mitochondrial content is not autophagy-dependent.

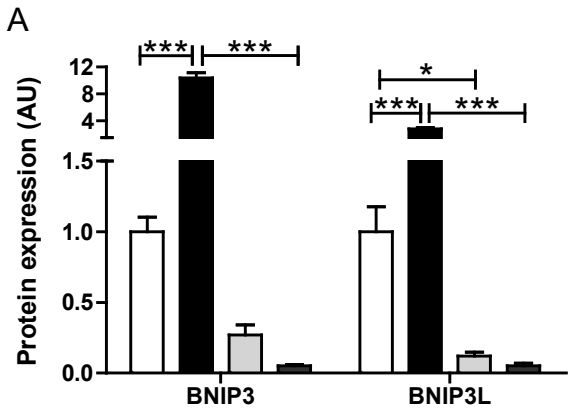

B

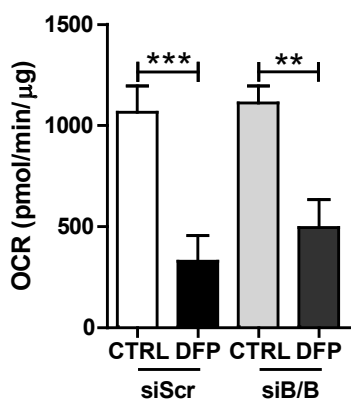

C

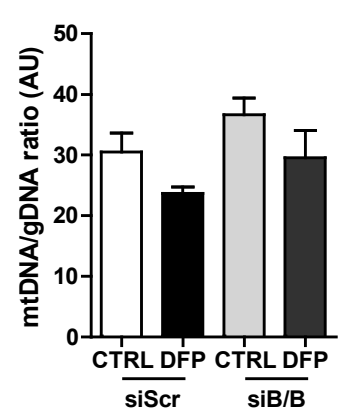

$\mathrm{CTRL}+$ siScr DFP + siScr

$\mathrm{CTRL}+\mathrm{siB} / \mathrm{B}$

$\mathrm{DFP}+\mathrm{siB} / \mathrm{B}$

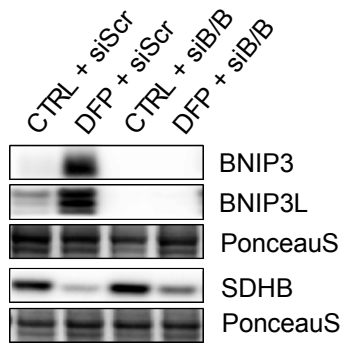

D

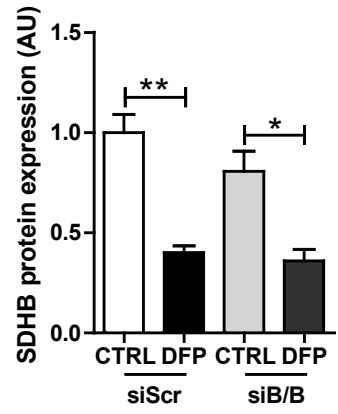

Figure 5: Knockdown of BNIP3 and BNIP3L does not attenuate DFP-induced loss of mitochondrial content and function. BNIP3 and BNIP3L protein levels $(n=3)(A)$, Maximal Oxygen Consumption Rate $(O C R)(n=8)(B)$, mtDNA/gDNA ratio $(n=3)(C)$, and SDHB protein levels $(n=3)(D)$ are depicted of myotubes exposed for $24 \mathrm{~h}$ to control (white bars), $300 \mu \mathrm{M}$ DFP (black bars), control + BNIP3/BNIP3L knockdown (light grey bars), or $300 \mu \mathrm{M}$ DFP + BNIP3/BNIP3L knockdown (dark grey bars). Data are depicted as mean + SEM, and significant differences are depicted compared with control or indicated bars $* p<0.05, * * p<0.01, * * * p<0.001$. siScr $=$ Scramble siRNA, $\mathrm{SiB} / \mathrm{B}=\mathrm{BNIP3} / \mathrm{BNIP} 3 \mathrm{~L}$ siRNA. 
Iron chelation-induced decrease in mitochondrial content and function is not BNIP3/BNIP3L-dependent

To test if the iron chelation-mediated decrease in mitochondrial content and function was dependent on activation of the mitophagy receptors BNIP3 and BNIP3L, we performed a siRNA-mediated knockdown of BNIP3 and BNIP3L and subsequently investigated SDHB protein levels, mtDNA copy number and cellular respiration in response to DFP treatment. The knockdown of BNIP3/BNIP3L resulted in abolishment of the basal as well as DFP-induced increase in BNIP3 and BNIP3L levels (Fig 5A). However, in line with our findings after chemical inhibition of autophagy, the knockdown of BNIP3/BNIP3L did not result in a rescue of either the loss of mitochondrial quantity or mitochondrial function in myotubes exposed to DFP (Fig 5B-D), suggesting that the iron chelation-induced decrease in mitochondrial content and function is not BNIP3/BNIP3L-dependent.

Iron chelation-induced decrease in mitochondrial content and function is not HIF1A-dependent

It is known that shortage of free iron results in HIF1A stabilization in several cell types, and HIF1A has been positioned as a major up-stream regulator of BNIP3 and BNIP3L expression $(20,26,27)$. To test if HIF1A plays a role in iron chelation-induced loss of mitochondrial content and function independently of BNIP3 and BNIP3L, we first studied if iron chelation induced HIF transcriptional activity, and we subsequently studied whether DFP-induced reductions in mitochondrial content and function were dependent on HIF1A in our model.

HIF transcriptional activity was potently increased in myotubes exposed to iron chelation (Fig 6A). Moreover, the siRNA-mediated knockdown of HIF1A (Fig 6B) resulted in the abolishment of the increase in BNIP3 and BNIP3L protein levels in myotubes exposed to DFP (Fig 6C). Knockdown of HIF1A however, did not rescue the loss of mitochondrial quantity nor mitochondrial respiration in myotubes exposed to DFP (Fig 6D-F).

Extracellular vesicles containing mitochondrial cargo appear after iron chelation Since our data showed that iron chelation-induced mitochondrial clearance was not dependent on autophagy, lysosomal breakdown, BNIP3/BNIP3L or HIF1A signalling, we subsequently investigated if iron chelation-induced mitochondrial clearance could also be executed via secretion of mitochondriacontaining EV. As depicted in Fig 7, myotubes exposed to iron chelation were found to secrete large amounts of EV in their extracellular milieu, while unexposed myotubes were not. These EV varied in size from $<100 \mathrm{~nm}$ to +/- $500 \mathrm{~nm}$ (Fig 7A-C). Neither knockdown of GABARAPL1, which has been implicated in both the intra-cellular trafficking of vesicles to the plasma 
membrane during hypoxia, and in mitochondrial breakdown $(28,29)$, nor knockdown of BNIP3/BNIP3L, which are both highly transcriptionally upregulated during iron chelation, prevented DFP-induced secretion of these EV as analysed with electron microscopy (Fig 7B-C). Interestingly, clear mitochondrial structures were detected in several of the larger EV by electron microscopy (Fig 7A).

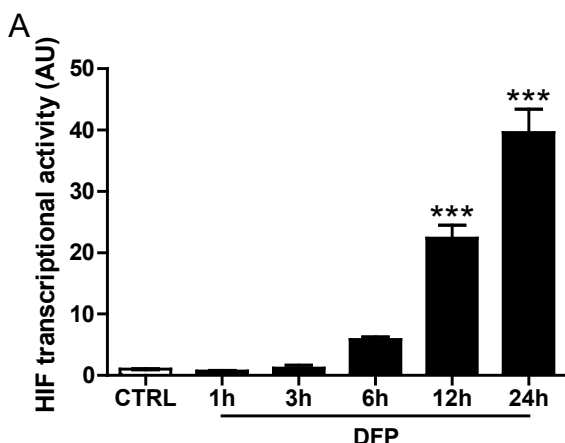

C

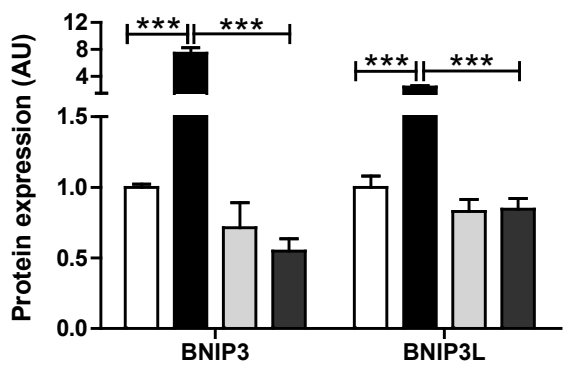

D

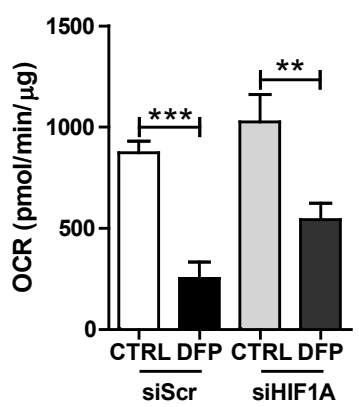

$\mathrm{E}$

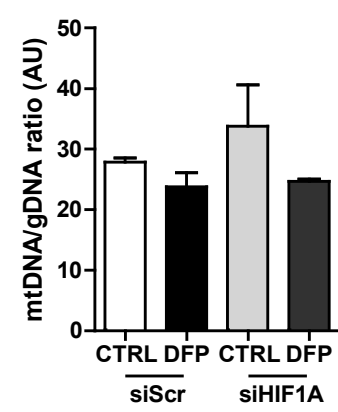

B

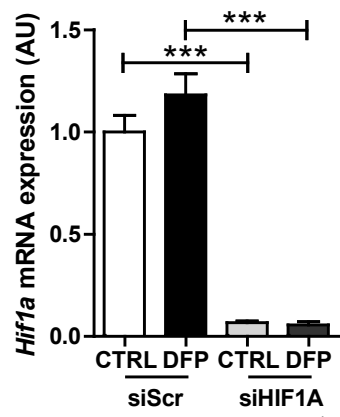

CTRL + siScr

$\mathrm{DFP}+$ siScr

CTRL + SIHIF1A

$\mathrm{DFP}+$ SiHIF1A

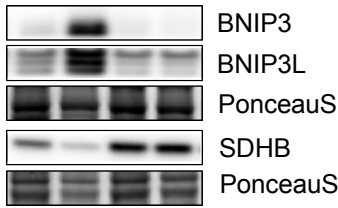

$\mathrm{F}$

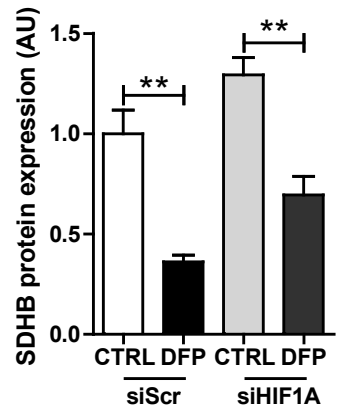

Figure 6: Knockdown of HIF1A does not attenuate DFP-induced loss of mitochondrial content and function. HIF transcriptional activity $(n=3)(A)$, Hif1a mRNA expression $(n=3)(B)$, BNIP3 and BNIP3L protein levels ( $n=3$ ) (C), Maximal Oxygen Consumption Rate (OCR) ( $=8)(D)$, mtDNA/gDNA ratio $(n=3)(E)$, and SDHB protein levels $(n=3)(F)$ are depicted of myotubes exposed for $24 \mathrm{~h}$ to control (white bars), $300 \mu \mathrm{M}$ DFP (black bars), control + HIF1A knockdown (light grey bars), or $300 \mu \mathrm{M}$ DFP + HIF1A knockdown (dark grey bars). Data are depicted as mean + SEM, and significant differences are depicted compared with control or indicated bars $* * p<0.01, * * * p<0.001$. siScr $=$ Scrambled siRNA, siHIF1A = HIF1A siRNA. 
A
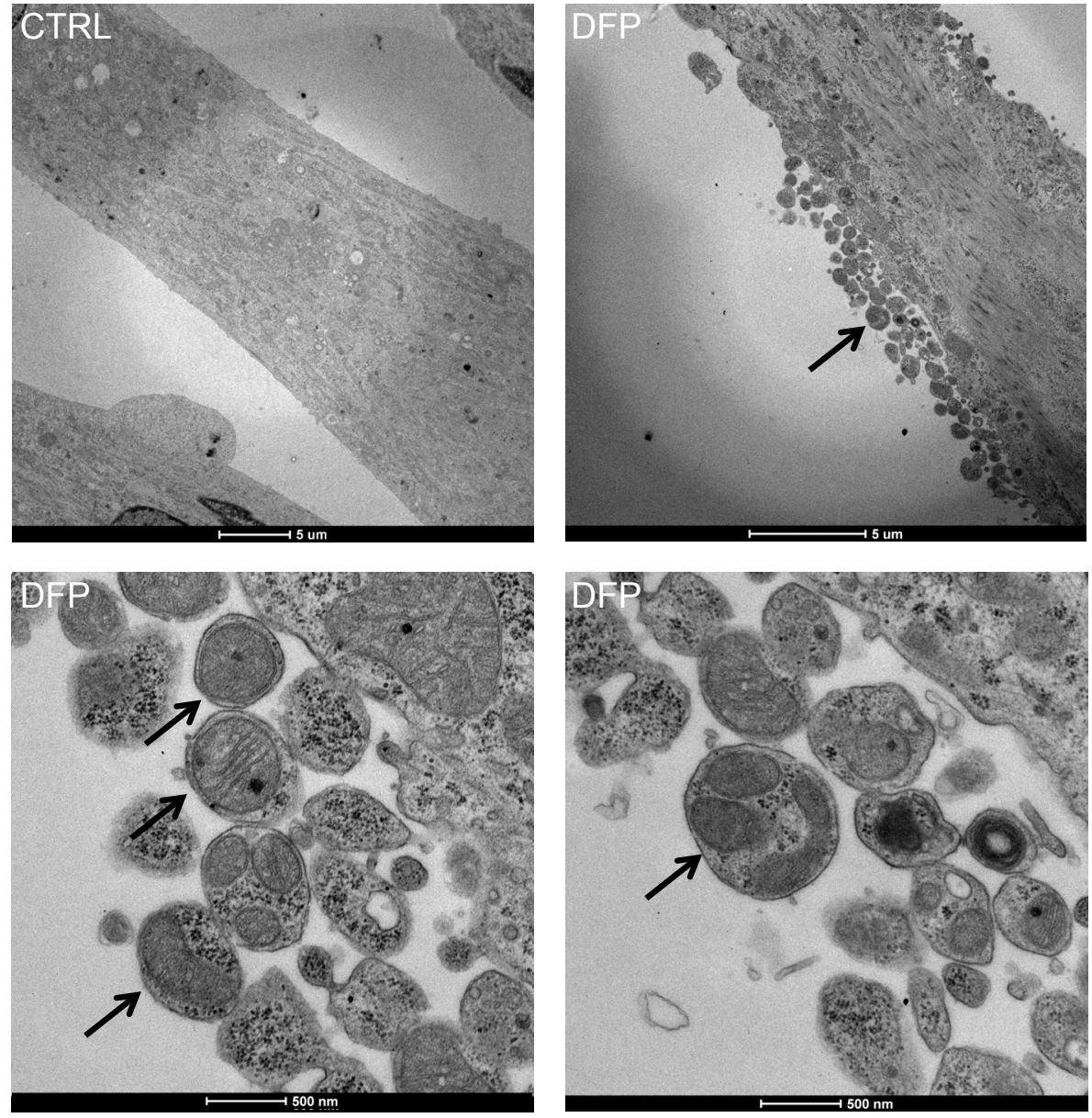

B

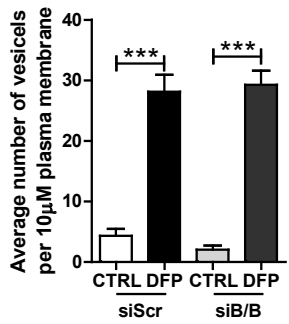

C

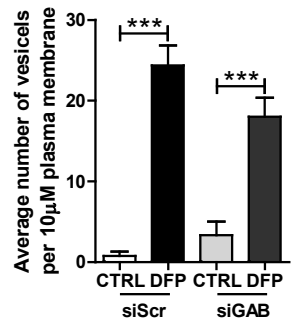

Figure 7: Iron chelation results in secretion of mitochondria-containing vesicles. Presence of mitochondrial-containing vesicles (black arrows) near the plasma membrane in iron chelationexposed myotubes (A). Quantification of vesicles in myotubes subjected to iron chelation in presence of scrambled, BNIP3/BNIP3L, or GABARAPL1 siRNA, 3 grids with 10 cells each were quantified (unless $<10$ cells were available on the grid) per condition (B, C). Data is depicted as mean + SEM, and significant differences are depicted $* * * p<0.001$. siScr $=$ Scramble siRNA, siB/B = BNIP3/BNIP3L siRNA, siGAB = GABARAPL1 siRNA. 
To verify this observation, EV were purified from $16,000 \times \mathrm{g}$ and $100,000 \times$ g pellets of DFP-exposed and unexposed myotubes, and both EV- and mitochondrial content markers were quantified. Indeed, a higher expression of the EV markers HSP70 and Flotillin-1, as well as higher levels of mitochondrial proteins and a higher mtDNA copy number were detected in the $16,000 \times \mathrm{g}$ EV fraction derived from DFP-exposed myotubes compared with unexposed myotubes (Fig 8A-B). These mitochondrial proteins were mainly detected in the $16,000 \times \mathrm{g}$, and not in the $100.00 \times$ g pellet (Fig 9).

A

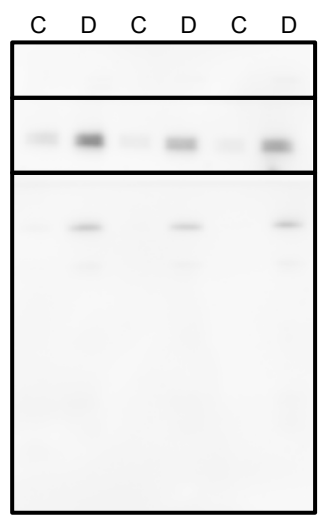

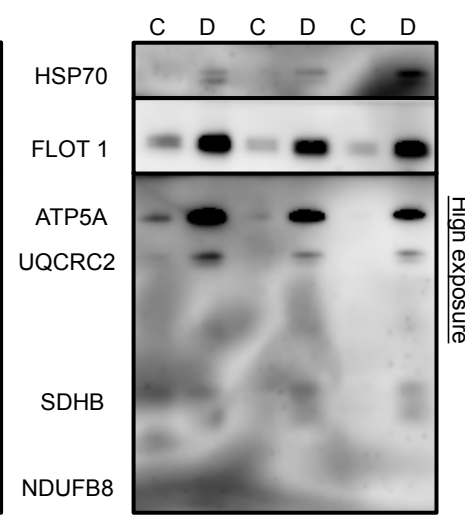

B

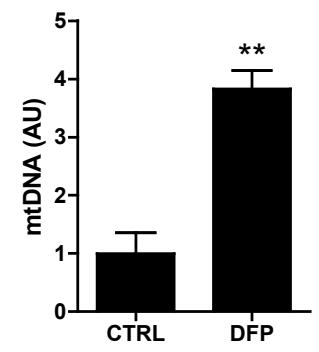

Figure 8: Mitochondrial constituents are present in extracellular vesicle fraction of myotubes subjected to iron chelation. Western blot for vesicle markers HSP70 and FLOT1, and mitochondrial components ATP5A, CQCRC2, SDHB and NDUFB8 in purified EV fraction from myotubes exposed for $24 \mathrm{~h}$ to control or $300 \mu \mathrm{M}$ DFP (A). mtDNA copy number in vesicle-fraction of myotubes exposed for $24 \mathrm{~h}$ to control or $300 \mu \mathrm{M}$ DFP (B). Data are depicted as mean + SEM ( $=3)$, and significant differences are depicted ${ }^{* *} p<0.01 . \mathrm{C}=$ unexposed, $\mathrm{D}=$ exposed to DFP.

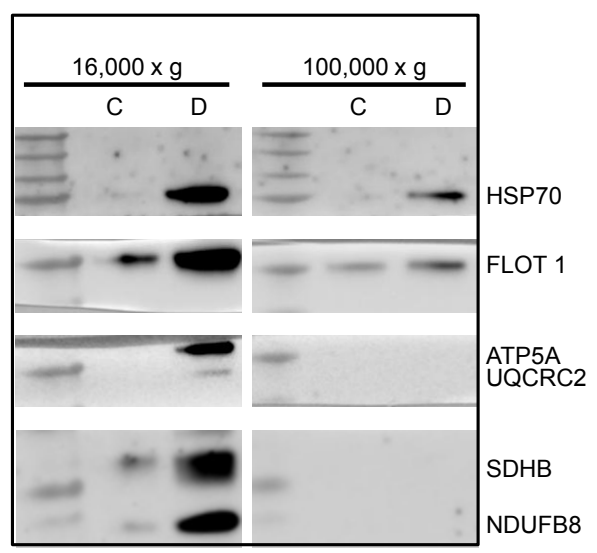

Figure 9: Mitochondrial constituents are present in $16,000 \times \mathrm{g}$ but not $100,000 \mathrm{x}$ g vesicle fraction of iron chelation-exposed myotubes. Western blot of purified $\mathrm{EV}$, from both $16,000 \times \mathrm{g}$ and $100,000 \times \mathrm{g}$ fraction, of myotubes exposed to $24 \mathrm{~h}$ of control or $300 \mu \mathrm{M}$ DFP. Vesicle markers HSP70 and FLOT1, and mitochondrial components ATP5A, CQCRC2, SDHB and NDUFB8 are depicted. $\mathrm{C}=$ unexposed, $\mathrm{D}=$ exposed to $300 \mu \mathrm{M}$ DFP. 


\section{Discussion}

This study is the first to show that iron deficiency in myotubes not only results in mitochondria surrounded by double membrane structures and mitochondriallysosomal co-localization, but also in increased expression levels of key proteins involved in mitophagy. Besides these indications for active mitophagy, iron chelation in myotubes in the current study also resulted in EV-mediated mitochondrial secretion in cultured myotubes. These changes were associated with decreased mitochondrial respiration and reductions in the abundance of specific mitochondrial proteins and mitochondrial DNA copy number. Experiments using pharmacological inhibitors of autophagy initiation or lysosomal degradation, or knockdown of specific components of the mitophagy machinery, revealed that these reductions were not dependent on general autophagy, BNIP3 and BNIP3L-mediated mitophagy, or HIF1A signalling. Mitochondrial secretion via EV might therefore represent an additional pathway for reducing mitochondrial content during iron deficiency. However, the exact mechanisms underlying the degradation of specific mitochondrial proteins as well as the molecular mechanisms and physiological relevance of the release of mitochondrial-containing vesicles in response to iron chelation in muscle remain to be elucidated.

From animal models, it is well-known that iron deficiency reduces mitochondrial function and content (2-7). Moreover, iron deficiency has been shown to have negative effects on exercise capacity and skeletal muscle performance in humans (reviewed in (7)). Collectively, this is in line with our data, as we clearly show that iron chelation induced a decline in mitochondrial respiration, and reduced mitochondrial DNA copy number in myotubes. However, although protein levels of SDHB and NDUFB8 (i.e. sub-units of OXPHOS complexes I and II) were reduced in response to iron chelation, HADH activity, CS activity and protein levels of CQCRC2 and ATP5A (i.e. subunits of OXPHOS complexes III and V) remained unaffected. Interestingly, other studies have also found that mitochondrial enzymes $(30-32)$ and OXPHOS complexes $(33,34)$ were unequally affected by iron deficiency in a similar manner. Although the simplest explanation for this discrepancy would be that iron chelation specifically targets ironcontaining, and not non-iron containing, proteins for breakdown or clearance, this does not completely explain our findings nor previously reported data. Indeed, although SDHB, NDUFB8, and CQCRC2 all contain iron, we observed that only SDHB and NDUFB8 were affected by iron chelation. It was previously proposed that iron sulphur clusters-based complexes are more sensitive to iron deficiency than haemoproteins (34), which might provide a logical explanation for our observations, since both SDHB and NDUFB8 contain iron sulphur 
clusters while CQCRC2 contains a haemoprotein. Moreover, it has been suggested that the Krebs cycle, of which CS is the rate-limiting enzyme, is upregulated in mitochondria exposed to iron deficiency to counteract the decreased electron flux in the electron transport chain $(31,32)$, which in our study could have compensated for any loss of CS activity. Combined, our results suggest that iron chelation not only decreases mitochondrial content, but also selectively targets mitochondrial proteins for degradation and hence changes mitochondrial composition. However, since this study did not focus on changes in mitochondrial composition, more research is needed to understand to what extent mitochondrial composition changes after iron chelation, and how this relates to decreased mitochondrial quantity and function.

In line with previous studies performed in non-skeletal muscle models (e.g. C. elegans and neuronal cells) (20-22), we are the first to report that mitochondrial clearance in increased in response to iron chelation in myotubes as well. Using electron microscopy, we found several double membrane-like structures enclosing mitochondria and we identified the presence of mitochondria in lysosomal-like structures in myotubes exposed to iron chelation. Furthermore, in line with a previous study using iron chelators in neuronal cells (20), protein levels of the transcriptionally-regulated mitophagy-receptors BNIP3 and BNIP3L were higher in response to iron chelation, while protein levels of the post-transcriptionally-regulated mitophagyreceptor FUNDC1 were lower. In addition, no changes in ubiquitin-mediated mitophagy-related transcription were observed in response to iron chelation. Together, these results indicate a role for receptor-mediated mitophagy in iron chelation-induced mitochondrial clearance.

Although these observations pointed towards increased receptor-mediated mitophagy after iron chelation, neither inhibition of autophagy initiation or lysosomal breakdown could prevent the loss of SDHB protein levels. Moreover, the specific knockdown of the mitophagy receptors BNIP3 and BNIP3L, or knockdown of HIF1A (which is known to be stabilized by iron chelation and to induce mitophagy via the BNIP3 pathway $(20,35)$ ), were unable to prevent the loss of mitochondrial respiration or SDHB protein levels as well. These data are in contrast with a previous report showing that the loss of mitochondrial quantity in SH-SY5Y cells in response to iron chelation is dependent on mitophagy, although these authors could likewise not prevent this with the siRNA-mediated knockdown of BNIP3 (20). In addition, this study also determined that iron chelation-induced mitophagy was PINK1/Parkin independent (20), which is in contrast to studies in $\mathrm{C}$. elegans, which reported that iron chelation-induced mitophagy is dependent on PINK/Parkin and BNIP3 (21). Our data implies 
that mitophagy might be present, but is not essential for the observed iron chelation-induced loss of mitochondrial content and function in myotubes, suggesting involvement of an alternative mechanism of mitochondrial clearance in the context of iron chelation.

Relevant in this context, we show for the first time that a large amount of EV-like structures are present near the plasma membrane of iron chelated myotubes. Furthermore, some of these vesicles appeared to contain mitochondrial cargo, or sometimes even apparent intact mitochondria. This suggests that mitochondria are being discarded from iron chelation-exposed myotubes via EV. Although myotubes have been reported to secrete $\mathrm{EV}$, and secretion of mitochondria via EV has previously been described in non-muscle cells, this is the first report describing mitochondria-containing EV in muscle cells $(10,11,36,37)$. As stated above, elimination of mitochondria through EV has recently been described as an alternative manner of mitochondrial clearance or horizontal mitochondrial transfer $(10,11)$. Since we showed that mitochondrial health is impaired after iron chelation, it could be speculated that, as an alternative to mitophagy, skeletal muscle cells donate impaired mitochondria to their environment where, possibly, cells (e.g. macrophages) that are better equipped to perform mitochondrial breakdown can clear them (10). Moreover, although we did not test if inhibition of autophagy or mitophagy resulted in an increased secretion of mitochondria-containing vesicles, it is even possible that the secretion of mitochondria is part of a pro-survival mechanism able to compensate for impaired mitophagy. Although no literature is present on the intracellular pathway leading towards iron chelation-induced vesicle secretion, we report that this pathway is independent of BNIP3 and BNIP3L, suggesting a dispensable role for receptor-mediated mitophagy signalling in this specific vesicle secretion. Moreover, we also report that it is independent of GABARAPL1, which has previously been positioned as a mediator of intracellular trafficking (28). Combined, our current results suggest that the secretion of mitochondrialcontaining EV might be an alternative, or additional, route to mitochondrial clearance under iron deprivation.

In conclusion, our results show that in myotubes exposed to iron chelation, mitochondria are packed into vesicles, are present in lysosomal structures, and are present in secreted vesicles in the extracellular space. Moreover, iron chelation results in reductions in mitochondrial content and function in myotubes, which are not autophagy-dependent. Although there are currently no methods available for specifically inhibiting EV secretion to study the causal relationship between the secreted mitochondria and the observed loss of mitochondrial quantity, our current results suggest a possible interplay between 
intracellular mitochondrial breakdown and mitochondrial secretion, which likely contribute to the mitochondrial impairments observed upon iron chelation. In order to gain new knowledge about the role of iron in the mitochondrial homeostasis, future studies should focus on elucidating the physiological relevance of EV-secreted mitochondria.

\section{Acknowledgements}

We would like to thank the Microscopy Core Lab of M4I at FHML of the University of Maastricht for the scientific and technological support in the microscopy work. 


\section{References}

1. Romanello V, Sandri M. Mitochondrial Quality Control and Muscle Mass Maintenance. Frontiers in physiology. 2015;6:422.

2. Ackrell BA, Maguire JJ, Dallman PR, Kearney EB. Effect of iron deficiency on succinate- and NADH-ubiquinone oxidoreductases in skeletal muscle mitochondria. The Journal of biological chemistry. 1984;259(16):10053-9.

3. Davies KJ, Donovan CM, Refino CJ, Brooks GA, Packer L, Dallman PR. Distinguishing effects of anemia and muscle iron deficiency on exercise bioenergetics in the rat. The American journal of physiology. 1984;246(6 Pt 1):E535-43.

4. Esteva S, Panisello P, Casas M, Torrella JR, Pages T, Viscor G. Morphofunctional responses to anaemia in rat skeletal muscle. Journal of anatomy. 2008;212(6):836-44.

5. Hagler L, Askew EW, Neville JR, Mellick PW, Coppes RI, Jr., Lowder JF, Jr. Influence of dietary iron deficiency on hemoglobin, myoglobin, their respective reductases, and skeletal muscle mitochondrial respiration. The American journal of clinical nutrition. 1981;34(10):2169-77.

6. Macdonald VW, Charache S, Hathaway PJ. Iron deficiency anemia: mitochondrial alpha-glycerophosphate dehydrogenase in guinea pig skeletal muscle. The Journal of laboratory and clinical medicine. 1985;105(1):11-8.

7. Stugiewicz M, Tkaczyszyn M, Kasztura M, Banasiak W, Ponikowski P, Jankowska EA. The influence of iron deficiency on the functioning of skeletal muscles: experimental evidence and clinical implications. European journal of heart failure. 2016.

8. Tkaczyszyn M, Drozd M, Wegrzynowska-Teodorczyk K, Flinta I, Kobak K, Banasiak $W$, et al. Depleted iron stores are associated with inspiratory muscle weakness independently of skeletal muscle mass in men with systolic chronic heart failure. Journal of cachexia, sarcopenia and muscle. 2018;9(3):547-56.

9. Barberan-Garcia A, Rodriguez DA, Blanco I, Gea J, Torralba Y, Arbillaga-Etxarri A, et al. Non-anaemic iron deficiency impairs response to pulmonary rehabilitation in COPD. Respirology. 2015;20(7):1089-95.

10. Phinney DG, Di Giuseppe M, Njah J, Sala E, Shiva S, St Croix CM, et al. Mesenchymal stem cells use extracellular vesicles to outsource mitophagy and shuttle microRNAs. Nature communications. 2015;6:8472.

11. Falchi AM, Sogos V, Saba F, Piras M, Congiu T, Piludu M. Astrocytes shed large membrane vesicles that contain mitochondria, lipid droplets and ATP. Histochemistry and cell biology. 2013;139(2):221-31.

12. Davis CH, Kim KY, Bushong EA, Mills EA, Boassa D, Shih T, et al. Transcellular degradation of axonal mitochondria. Proceedings of the National Academy of Sciences of the United States of America. 2014;111(26):9633-8.

13. Palikaras K, Tavernarakis N. Mitochondrial homeostasis: The interplay between mitophagy and mitochondrial biogenesis. Experimental gerontology. 2014(56):182-8.

14. Yamaguchi O, Murakawa T, Nishida K, Otsu K. Receptor-mediated mitophagy. Journal of molecular and cellular cardiology. 2016(95):50-6.

15. Wei $H$, Liu L, Chen $Q$. Selective removal of mitochondria via mitophagy: distinct pathways for different mitochondrial stresses. Biochimica et biophysica acta. 2015;1853(10 Pt B):2784-90. 
16. Stotland A, Gottlieb RA. Mitochondrial quality control: Easy come, easy go. Biochimica et biophysica acta. 2015;1853(10 Pt B):2802-11.

17. Matsuda N. Phospho-ubiquitin: upending the PINK-Parkin-ubiquitin cascade. Journal of biochemistry. 2016;159(4):379-85.

18. Lazarou M, Sliter DA, Kane LA, Sarraf SA, Wang C, Burman JL, et al. The ubiquitin kinase PINK1 recruits autophagy receptors to induce mitophagy. Nature. 2015;524(7565):309-14.

19. Eiyama A, Okamoto K. PINK1/Parkin-mediated mitophagy in mammalian cells. Current opinion in cell biology. 2015;33:95-101.

20. Allen GF, Toth R, James J, Ganley IG. Loss of iron triggers PINK1/Parkin-independent mitophagy. EMBO reports. 2013;14(12):1127-35.

21. Schiavi A, Maglioni S, Palikaras K, Shaik A, Strappazzon F, Brinkmann V, et al. Iron-Starvation-Induced Mitophagy Mediates Lifespan Extension upon Mitochondrial Stress in C. elegans. Current biology : CB. 2015;25(14):1810-22.

22. Nagi M, Tanabe K, Nakayama H, Ueno K, Yamagoe S, Umeyama T, et al. Iron-depletion promotes mitophagy to maintain mitochondrial integrity in pathogenic yeast Candida glabrata. Autophagy. 2016;12(8):1259-71.

23. Van Deun J, Mestdagh P, Sormunen R, Cocquyt V, Vermaelen K, Vandesompele J, et al. The impact of disparate isolation methods for extracellular vesicles on downstream RNA profiling. Journal of extracellular vesicles. 2014;3.

24. Lotvall J, Hill AF, Hochberg F, Buzas El, Di Vizio D, Gardiner C, et al. Minimal experimental requirements for definition of extracellular vesicles and their functions: a position statement from the International Society for Extracellular Vesicles. Journal of extracellular vesicles. 2014;3:26913.

25. Slot IG, Schols AM, de Theije CC, Snepvangers FJ, Gosker HR. Alterations in Skeletal Muscle Oxidative Phenotype in Mice Exposed to 3 Weeks of Normobaric Hypoxia. Journal of cellular physiology. 2016;231(2):377-92.

26. Bellot G, Garcia-Medina R, Gounon P, Chiche J, Roux D, Pouyssegur J, et al. Hypoxia-induced autophagy is mediated through hypoxia-inducible factor induction of BNIP3 and BNIP3L via their BH3 domains. Molecular and cellular biology. 2009;29(10):2570-81.

27. Wang X, Yang H, Yanagisawa D, Bellier JP, Morino K, Zhao S, et al. Mitochondrial ferritin affects mitochondria by stabilizing HIF-1alpha in retinal pigment epithelium: implications for the pathophysiology of age-related macular degeneration. Neurobiology of aging. 2016;47:168-79.

28. Keulers TG, Schaaf MB, Peeters HJ, Savelkouls KG, Vooijs MA, Bussink J, et al. GABARAPL1 is required for increased EGFR membrane expression during hypoxia. Radiotherapy and oncology : journal of the European Society for Therapeutic Radiology and Oncology. 2015;116(3):417-22.

29. Boyer-Guittaut M, Poillet L, Liang Q, Bole-Richard E, Ouyang X, Benavides GA, et al. The role of GABARAPL1/GEC1 in autophagic flux and mitochondrial quality control in MDA-MB-436 breast cancer cells. Autophagy. 2014;10(6):986-1003.

30. Ohira Y, Cartier LJ, Chen M, Holloszy JO. Induction of an increase in mitochondrial matrix enzymes in muscle of iron-deficient rats. The American journal of physiology. 1987;253(5 Pt 1):C639-44. 
31. Willis WT, Brooks GA, Henderson SA, Dallman PR. Effects of iron deficiency and training on mitochondrial enzymes in skeletal muscle. J Appl Physiol (1985). 1987;62(6):2442-6.

32. Cartier LJ, Ohira Y, Chen M, Cuddihee RW, Holloszy JO. Perturbation of mitochondrial composition in muscle by iron deficiency. Implications regarding regulation of mitochondrial assembly. The Journal of biological chemistry. 1986;261(29):13827-32.

33. Evans TC, Mackler B. Effect of iron deficiency on energy conservation in rat liver and skeletal muscle submitochondrial particles. Biochemical medicine. 1985;34(1):93-9.

34. Galy B, Ferring-Appel D, Sauer SW, Kaden S, Lyoumi S, Puy H, et al. Iron regulatory proteins secure mitochondrial iron sufficiency and function. Cell metabolism. 2010;12(2):194-201.

35. Zhang $H$, Bosch-Marce M, Shimoda LA, Tan YS, Baek JH, Wesley JB, et al. Mitochondrial autophagy is an HIF-1-dependent adaptive metabolic response to hypoxia. The Journal of biological chemistry. 2008;283(16):10892-903.

36. Forterre A, Jalabert A, Berger E, Baudet M, Chikh K, Errazuriz E, et al. Proteomic analysis of $\mathrm{C} 2 \mathrm{C} 12$ myoblast and myotube exosome-like vesicles: a new paradigm for myoblast-myotube cross talk? PloS one. 2014;9(1):e84153.

37. Kim S, Lee MJ, Choi JY, Park DH, Kwak HB, Moon S, et al. Roles of Exosome-Like Vesicles Released from Inflammatory C2C12 Myotubes: Regulation of Myocyte Differentiation and Myokine Expression. Cellular physiology and biochemistry : international journal of experimental cellular physiology, biochemistry, and pharmacology. 2018;48(5):1829-42. 


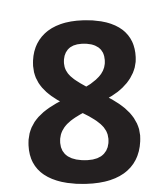

General discussion 


\section{General discussion}

The main objective of the research described in this thesis was to study if chronic obstructive pulmonary disease (COPD)-related manifestations muscle disuse, systemic inflammation, and more speculatively, iron deficiency, result in the onset of mitochondrial clearance, and consequently in the loss of muscle oxidative capacity. In this chapter, the research presented in this thesis is positioned in a broader context including identification and discussion of leads for further in depth investigation.

\section{Skeletal muscle mitochondrial clearance in COPD and beyond}

It has been well-established that patients with COPD, as well as patients with congestive heart failure (CHF) and type 2 diabetes (T2D) suffer from a loss of skeletal muscle oxidative capacity (1-9). Although several previous studies have described decreased expression of mitochondrial biogenesis regulators in skeletal muscle of patients with these chronic diseases (10-14), little is known about mitochondrial clearance. In a study addressing the role of autophagy in skeletal muscle wasting, Guo et al. reported some data indicative of mitochondrial breakdown in vastus lateralis of COPD patients (15), and Kruse et al. reported no differences in autophagy- and mitophagy-related markers in the vastus lateralis of obese and T2D subjects compared with healthy subjects (16). Although mitochondrial impairments are also present in skeletal muscle of $\mathrm{CHF}$ patients $(4,8)$, there is currently no data available on skeletal muscle mitophagy in these patients. Although the studies of Guo and Kruse both reported markers involved in the regulation of mitochondrial breakdown, they provide no proof for actual increased mitochondrial breakdown in the skeletal muscle of these patients $(15,16)$. Indeed, the measurement of the actual process of mitochondrial breakdown via mitophagy (i.e. the engulfment of mitochondria by autophagosomes and their subsequent breakdown in lysosomes; mitophagic flux) is challenging in in vivo studies, which is extensively discussed in the "limitations of measurements of mitochondrial clearance" section below.

In the current thesis, we reported molecular signalling patterns indicative of increased mitochondrial breakdown in skeletal muscle of patients with COPD, characterized by loss of muscle oxidative capacity (chapter $\mathbf{3}$ ). These results are generally in line with the conclusions of Guo et al, with the added value of our study being that we studied a much larger COPD patient population and more importantly, included measurements for mitochondrial quantity which enabled us to directly correlate indices of mitophagy with loss of mitochondrial content. Although this still provides no direct evidence of increased mitophagic flux per se, these findings strongly point towards a role for mitochondrial breakdown 
in the loss of mitochondrial content. The fact that Kruse et al. found no indications of enhanced mitophagy-signalling suggests that skeletal muscle mitochondrial breakdown plays a more important role in COPD compared with T2D, although more studies are needed to establish this, especially since the study of Kruse et al. did not include measurements for mitochondrial content (16).

In conclusion, research on mitochondrial clearance in the skeletal muscle of patients with chronic diseases is just starting to emerge, and current studies indicate that skeletal muscle mitophagy-regulation is increased in COPD. Whether this is also true for other chronic disorders such as T2D and CHF requires further investigation. Furthermore, future studies should preferably include continuous, or at least multiple, measurements of mitochondrial content and function, to establish a net mitochondrial loss or gain per patient, which can be associated with the individual mitophagy status. Ideally, these measurements can, combined, be used as a reasonably reliable surrogate marker of mitophagic flux, which is currently probably the second best option in a clinical setting.

\section{Pitfalls in the translation of mitochondrial clearance in models of systemic inflammation, muscle disuse, and iron deficiency to COPD}

Systemic inflammation, decreased physical activity, and iron deficiency are common in COPD patients and may, on their own or synergistically, affect mitochondrial homeostasis. As these triggers are not COPD-specific, they could also drive mitochondrial impairments in patients with other chronic diseases (chapter 2). We used several approaches to model these triggers in an isolated setting, and discuss their results in relation to the clinical setting in the following section.

\section{Systemic inflammation}

To study the role of systemic inflammation on skeletal muscle mitochondrial clearance, we used two approaches. First, we divided our patient cohort in patients with low- and high-CRP blood levels, and compared the skeletal muscle molecular signalling constituents between these groups. Secondly, we employed a murine model for pulmonary-induced systemic inflammation, the intratracheal instillation of lipopolysaccharide (IT-LPS) model (17), and measured these same skeletal muscle molecular signalling constituents over time after the induction systemic inflammation in this model.

In this thesis, we found that low-grade systemic inflammation was associated with increased BNIP3 and autophagy-related protein levels in muscle of COPD patients (chapter 3). Moreover, we showed that molecular signalling of mitophagy and autophagy was temporally increased, while transcriptional 
regulation of mitochondrial biogenesis was temporally decreased after acute induction of systemic inflammation (chapter 4). Combined, we report that in both the human and murine studies increased BNIP3 and autophagy-related molecular signalling in skeletal muscle was associated with the presence and induction of systemic inflammation.

Despite these similarities, there are some clear differences between the IT-LPS model and the clinical situation. Most importantly, patients with clinically stable COPD have chronic low-grade systemic inflammation whereas the systemic inflammation induced in our murine model is acute, temporal, and much more severe (18). Indeed, fold changes in observed molecular signalling (e.g. BNIP3 and BNIP3L protein and mRNA expression) between IT-LPS exposed mice and vehicle control mice were much more robust compared to the observed differences between high- and low-CRP COPD patients. To obtain more representative information for clinically stable COPD patients in future research, murine models for pulmonary-induced chronic inflammation are available, of which e.g. the chronic smoke exposure model (19) and the genetically altered SP-C/TNFa model (20) both develop low-grade systemic inflammation (21). Although the pulmonary compartment is thought to be a major initiator of systemic inflammation in COPD patients (22), other tissues are likely to contribute to its development as well. For example, adipose tissue has previously been identified as a possible source of systemic inflammation in COPD patients (23), and murine models of adipose tissue-induced systemic inflammation could therefore provide additional knowledge on the role of systemic inflammation in mitochondrial clearance. Moreover, as adipose-tissue induced systemic inflammation is also present in other diseases like obesity and T2D, these studies might yield new insights for these disorders as well (24).

Interestingly, we did not find any systemic-inflammation associated differences in mitochondrial content in both the human and murine study, despite differences in molecular markers of mitochondrial breakdown signalling. Although this suggests that the presence of systemic inflammation does not participate in the loss of mitochondrial content, it is also possible that the studied inflammatory insults were not long (in case of the murine study) or severe (in case of the clinical study) enough to develop a loss of mitochondrial content. Indeed, muscle inflammatory signalling was only temporary increased in our murine model and returned back to baseline within $48 \mathrm{~h}$ after IT-LPS. Moreover, in more severe models of systemic inflammation, Mofarrahi et al. showed that mice exposed to intraperitoneal (i.p.) injection of $20 \mathrm{mg} / \mathrm{kg}$ LPS developed ultrastructural injuries in the mitochondria of skeletal muscle (25), Piquereau et al. found decreased activity of cardiac muscle oxidative phosphorylation complex I 
and II after i.p. injection of $5 \mathrm{mg} / \mathrm{kg}$ LPS (26), and Tanaka et al. found decreased muscle CS and SDH activity after 4 executive days of i.p. injection of $10 \mathrm{mg} / \mathrm{kg}$ LPS (27). All these studies were performed with i.p. injection, which delivers the LPS directly in the system in contrast to our IT-LPS instillation. Furthermore, all these studies used a multitude of the dose that we have used. In conclusion, our results suggest that the effect of short-term or low-grade systemic inflammation on the mitochondrial content is limited, despite molecular signalling patterns indicative of increased mitochondrial clearance.

\section{Muscle disuse}

To study the role of muscle disuse on skeletal muscle mitochondrial clearance, we used two models. First, we subjected mice to 3 days of hind-limb suspension, where the hind-limbs were completely unloaded, and studied if skeletal muscle molecular signalling constituents were different compared to control. Secondly, we used a 7-day leg-cast on healthy men, and compared skeletal muscle molecular signalling constituents before and after the casting period.

There are multiple models for skeletal muscle disuse, of which muscle immobilisation, denervation, and suspension are the most common. In these models, disuse is a well-established initiator of skeletal muscle mitochondrial impairments including decreased oxidative capacity (28-37), decreased mitochondrial biogenesis $(30-35,38,39)$, and increased mitochondrial clearance $(31,36,37,40)$. Since most of these studies were performed with prolonged muscle unloading, the effects of short-term unloading remained relatively understudied. The results reported in this thesis (chapter 5) emphasize the early-onset of acute disuse-induced mitochondrial remodelling in skeletal muscle. Moreover, our results also indicate that this skeletal muscle mitochondrial remodelling is already present in humans exposed to short periods of complete muscle disuse, and is therefore relevant for virtually all hospitalized bed-bound patients with any disease.

Since our study was performed in acute disuse models based on complete unloading of muscle, while COPD patients with clinically stable disease usually suffer from physical inactivity-associated decreased muscle use, translation of these results to the clinical situation should be done with caution. Moreover, literature on the regulation of mitochondrial clearance in relation to the muscle disuse in COPD patients is currently lacking. To establish the association between mitochondrial clearance and the level of muscle disuse in clinically stable COPD patients, studies with extensive physical activity monitoring should be combined with measurements of muscle mitochondrial content and clearance. 


\section{Iron deficiency}

Iron deficiency is associated with impaired exercise capacity in $\operatorname{COPD}(41,42)$ and CHF patients $(43,44)$, and could very well be an important factor involved in the loss of oxidative capacity in these diseases as proposed in chapter $\mathbf{2}$, and more recently by others as well (45). To study the role of iron deficiency on mitochondrial clearance in skeletal muscle, we used two approaches. First, we divided our patient cohort in patients with and without systemic iron deficiency, and compared the skeletal muscle mitochondrial breakdown signalling constituents between these groups. Secondly, we performed an in vitro study in which we modelled iron deficiency using the iron chelating reagent deferiprone.

In this thesis we found that iron deficient COPD patients had lower skeletal muscle BNIP3L protein levels compared with non-iron deficient COPD patients, resembling the levels of healthy controls, in addition to higher BNIP3 and LC3B gene- but not protein-expression (chapter 3 ). This is in contrast with our in vitro report, where we found increased levels of both BNIP3 and BNIP3L protein in cultured skeletal muscle myotubes subjected to iron chelation (chapter 7).

Although our in vitro model of iron chelation-induced iron deficiency is not directly translatable to iron deficient COPD patients, the opposite changes in BNIP3L protein levels in muscle of iron deficient patients compared with the in vitro iron-deprived myotubes are remarkable and difficult to explain. As we studied iron deficiency as an isolated trigger in vitro, it is likely that iron deficiency indeed results in increased BNIP3 and BNIP3L expression in conditions of acute and absolute iron deficiency. Indeed, these results are highly comparable to similar studies in different cell lines (46). In our COPD patient cohort, iron deficiency was diagnosed according to clinical criteria based on blood ferritin levels and transferrin saturation. It is unknown if and how such chronic and systemic iron deficiency can be translated to iron metabolism locally in skeletal muscle, which makes it important to establish the intramuscular iron content in future studies (44). In conclusion, although we provide clear evidence that isolated and absolute iron deficiency induces mitochondrial clearance in myotubes, better controlled studies are needed to study if iron deficiency results in increased mitochondrial breakdown in skeletal muscle of COPD patients as well.

\section{Acute and synergistic effects}

Many COPD patients suffer from COPD exacerbations, which are defined by a worsening of the patient's respiratory symptoms that is beyond normal day-to-day variations and leads to a change in medication (47). These exacerbations are known for a significant and rather acute increase in pulmonary- and systemic- 
inflammation $(47,48)$. Moreover, exacerbating patients feel sick and may even need to be hospitalised, aggravating their muscle disuse due to a further reduction in physical activity levels or bed-confinement respectively $(4,49,50)$. With regard to iron deficiency in COPD patients, a prevalence of $18 \%$ (FEV1 of $40 \%$ predicted) was reported by Nickol et al. (51), and 30\% (FEV1 of $40 \%$ predicted) was reported by us (chapter $\mathbf{3}$ ) in clinically stable patients, while Silverberg et al. reported that $44 \%$ of the patients hospitalized for a COPD exacerbations were anaemic, of which $100 \%$ were iron deficient (52). Together, this suggests that iron deficiency is more prevalent in exacerbating COPD patients. However, since iron status was only measured in $3 \%$ of the nonanaemic and $38 \%$ anaemic patients in the study of Silverberg et al., better controlled studies are needed to prove this observation (52). Of note, the diagnosis of iron deficiency is highly influenced by systemic inflammation within these patients, which makes it difficult to study them completely separately in this setting $(42,53)$. For example, inflammatory processes are able to limit iron flux through the circulation and disturb the strong correlation that exists between serum ferritin and total iron storage in physiological conditions (53). Nevertheless, as systemic inflammation, muscle disuse, and iron deficiency acutely converge and intensify during COPD exacerbations, and because our experimental models were relatively acute and severe of nature, it is possible that these models more closely resemble an acute exacerbation rather than the stable phase of the disease. It is therefore feasible that mitochondrial clearance is especially associated to the unstable phase, especially because the synergistic presence of extra-pulmonary events during COPD exacerbations results in lasting impairments of muscle mass and function. It was therefore proposed that these exacerbations provide a window-ofopportunity to limit the detrimental effects of these events (54).

\section{Mitochondrial clearance via extracellular vesicular secretion}

Skeletal muscle has been identified as a secretory organ during the past decade, secreting many different kinds of products which exert autocrine, paracrine and endocrine effects (55). It was reported that at least part of the secrotome consists of extracellular vesicles (EV), i.e. small (100-1000 $\mu \mathrm{m})$ cargo-holding double-membrane structures secreted from the plasma membrane, in both murine muscle as well as in vitro cultured $\mathrm{C} 2 \mathrm{C} 12$ cells (56-58). In chapter 7 , we are the first to describe a high amount of EV-like structures near the plasma membrane of iron-deprived myotubes. Furthermore, some of these vesicles seemed to contain intact mitochondria, which suggests that mitochondria are being discarded from the iron chelation exposed myotubes via EV. 
Mitochondria-containing EV have recently been described in non-skeletal muscle models as an alternative for mitophagy. In detail, Phinney et al. reported that mesenchymal stem cells were able to donate impaired mitochondria to their environment, after which they were taken up by macrophages, which are better equipped to perform mitochondrial breakdown (59). Moreover, Davis et al. found that mitochondria can be 'shedded' from ganglion cell axons in order to outsource mitophagy to other cell-types as well (60). In line with the above described studies, our results could be indicative of mitochondria-containing EV secretion as alternative route for mitochondrial clearance, in addition or instead of mitophagy. Apart from being an alternative to mitochondrial breakdown, Falchi et al. suggested that astrocyte-secreted mitochondrialcontaining EV could be functionally reintegrated into acceptor-cells, boosting their mitochondrial capacity (61). However, it is unlikely that in our in vitro experiments in which myotubes are exposed to iron deprivation, mitochondria-containing EV are secreted in order to recover or maintain mitochondrial health elsewhere. Nevertheless, based on the concept that muscle cells can secrete mitochondria via EV one could speculate that this would work the other way around as well, implying that skeletal muscle cells are able to receive and integrate healthy mitochondria from extracellular sources, thereby restoring mitochondrial capacity in these cells. In conclusion, we are the first to describe mitochondria in skeletal muscle-derived $\mathrm{EV}$, which provides a novel paradigm in skeletal muscle cell plasticity research.

\section{Limitations of mitochondrial clearance measurements}

As described above, the measurement of actual mitochondrial breakdown via mitophagic flux is challenging in in vivo studies. Most importantly, this is due to the fact that the final step of mitophagy is the lysosomal breakdown of the mitochondrion-containing autophagosome, including most mitophagy- or autophagy-mediating proteins that are involved in the process. As an increased transcriptional and translational regulation of mitophagy, and inhibition of lysosomal degradation can both result in accumulation of mitophagy- and autophagy-specific constituents, accumulation of these constituents can be indicative of impairment degradation as well as increased initiation (62). So although gene and protein expression levels of key mitophagy mediators are valuable indicators of mitochondrial breakdown, actual breakdown itself cannot be proven by a single measurement of gene and protein expression only (62).

To be able to measure the actual flux through the clearance pathway, several experimental possibilities have been developed. First, the quantity of mitochondrial- and breakdown-specific constituents could be compared in conditions where lysosomal inhibition is present or absent during a 
period of time (where the difference in quantity then represents the lysosomalbreakdown over that specific time period) (62). Several compounds are available as clinically approved drugs for blocking lysosomal breakdown, e.g. chloroquine and hydroxychloroquine, but they have serious side effects which limits their applicability in clinical studies (63). Moreover, although these compounds have been used in animal studies in the past, they have been shown to be inducers of autophagy by themselves (62). Secondly, live-monitoring of the co-localisation of mitochondria with autophagosomes or lysosomes would provide actual proof of mitophagy as well, but no in vivo live mitophagy monitoring can be performed in human skeletal muscle at this moment. Currently, new possibilities are slowly evolving for measuring mitophagy in in vivo studies. For example, there are animal models in which mitochondria are tagged by tandem fluorescent dyes, of which one is quenched by low $\mathrm{pH}$ while the other is resistant to low $\mathrm{pH}$. When imaging these mitochondria, the detection of both dyes together indicates that the mitochondria are in a normal pH environment, while the detection of just the acid-resistant dye indicates that the mitochondria are located in lysosomes. The use of these models will limit animal numbers and between-animal variability in future studies (64).

To overcome these problems as good as possible, the simultaneous quantification of the mitochondrial content (as a surrogate marker for net mitophagy result) in addition to the mitophagy- as well as autophagy-related proteins provides information about the overall effect of the pathway and is an indication of the consequences of altered mitophagic flux. Sequential measurements in one subject would even provide information about the net change in individual mitochondrial content, which would highly benefit the accuracy of the assessment of changes in general mitochondrial content. However, these measurements can currently only be performed in invasive muscle biopsies which provides ethical limitations (62).

\section{Mitochondrial clearance in skeletal muscle, friend or foe?}

Although mitochondrial breakdown may lead to loss of mitochondrial content, it is also crucial for the maintenance of a healthy mitochondrial network (65). Indeed, mitophagy was initially described as a mechanism responsible for the clearance of dysfunctional mitochondria (66), and impaired mitophagy has been associated with decreased mitochondrial functioning and increased generation of reactive oxygen species (65). Furthermore, several studies report that inhibition of mitophagy ultimately results in impaired muscle function $(67,68)$. Moreover, mitophagy has often been described as part of mitochondrial remodelling during exercise-induced increase in oxidative capacity $(69,70)$. Interestingly, our group has recently shown that mitophagy signalling is part of the molecular signal- 
ling adaptation-response of skeletal muscle during physical therapy in patients with COPD (71). Furthermore, in chapter 6 we showed through the combination of a cluster analysis and high-resolution time-course study, that the molecular regulation of protein turnover, mitochondrial turnover (including mitophagy), and myonuclear turnover are correlated and temporally associated during recovery from muscle atrophy. These results suggest an interdependency or joint regulation of these muscle mass- and oxidative metabolism-related processes during recovery from atrophy and indicate that mitochondrial clearance is also important during periods of skeletal muscle recovery.

Together with the above described literature showing that mitochondrial clearance is highly relevant for maintenance of mitochondrial quality, the currently described association between mitochondrial breakdown and muscle recovery (chapter 6) further underlines that mitochondrial clearance may be indispensable for healthy skeletal muscle function. Indeed, as a main indicator of dysfunctional mitochondria (i.e. excess mitochondrial reactive oxygen species production) was previously shown to be increased in skeletal muscle of COPD patients (72), it could very well be that patterns of mitochondrial breakdown in skeletal muscle of COPD patients described in this thesis (chapter 3) function to clear dysfunctional mitochondria. Furthermore, as systemic inflammation, muscle disuse and iron deficiency are known to induce mitochondrial impairments $(44,73,74)$, mitochondrial clearance could prevent accumulation of dysfunctional mitochondria in these conditions as well, thereby protecting the muscle from further damage.

In conclusion, although our research identified mitochondrial clearance pathways as potential therapeutic targets in disorders characterized by loss of skeletal muscle mitochondrial capacity, it is likely that inhibition strategies might influence the overall health of the mitochondrial pool. To develop intervention strategies that target only pathological effects without interfering with normal mitochondrial homeostasis, it is essential to obtain additional information about the upstream mechanisms and triggers leading to mitochondrial dysfunction and mitochondrial clearance. If dysfunctional mitochondrial by themselves are triggers for mitochondrial clearance, prevention of mitochondrial dysfunction could be preferable over the inhibition of mitochondrial clearance per se. Lifestyle modifications, as well as nutritional or physical interventions targeting systemic inflammation, muscle disuse, or iron status could prove beneficial as upstream mitochondrial-clearance limiting and mitochondrial content-preserving therapies. 


\section{Conclusion}

This thesis shows that molecular signalling indicative of mitochondrial clearance is present in skeletal muscle of COPD patients who suffer from loss of muscle oxidative capacity. Moreover, systemic inflammation, muscle disuse, recovery of muscle disuse, and iron deficiency can individually contribute to the molecular (dys-)regulation of skeletal muscle mitochondrial clearance. However, since mitophagy is also part of the skeletal muscle recovery process, interfering with mitochondrial clearance is not recommended as oxidative capacity preserving therapy. Therefore, future research should be aimed to unravel if mitochondrial clearance-induced loss of oxidative capacity can be prevented using lifestyle modifications as well as nutritional or physical interventions, rather than pharmacological interventions. The successive monitoring of skeletal muscle composition and function, and early recognition of the loss of oxidative capacity are instrumental for this goal. Moreover, the role of mitochondrial-containing vesicles as part of the mitochondrial clearance machinery needs to be unravelled. 


\section{References}

1. van den Borst B, Gosker HR, Schols AM. Central fat and peripheral muscle: partners in crime in chronic obstructive pulmonary disease. American journal of respiratory and critical care medicine. 2013 Jan 1;187(1):8-13. PubMed PMID: 23281350.

2. Adams V, Doring C, Schuler G. Impact of physical exercise on alterations in the skeletal muscle in patients with chronic heart failure. Frontiers in bioscience : a journal and virtual library. 2008;13:302-11. PubMed PMID: 17981548.

3. Remels $A H$, Gosker HR, Langen RC, Polkey M, Sliwinski P, Galdiz J, et al. Classical NF-kappaB activation impairs skeletal muscle oxidative phenotype by reducing IKK-alpha expression. Biochimica et biophysica acta. 2014 Feb;1842(2):175-85. PubMed PMID: 24215713.

4. Maltais F, Decramer M, Casaburi R, Barreiro E, Burelle Y, Debigare R, et al. An official American Thoracic Society/European Respiratory Society statement: update on limb muscle dysfunction in chronic obstructive pulmonary disease. American journal of respiratory and critical care medicine. 2014 May 1;189(9): e15-62. PubMed PMID: 24787074. Pubmed Central PMCID: 4098112.

5. Mogensen M, Sahlin K, Fernstrom M, Glintborg D, Vind BF, Beck-Nielsen H, et al. Mitochondrial respiration is decreased in skeletal muscle of patients with type 2 diabetes. Diabetes. 2007 Jun;56(6):1592-9. PubMed PMID: 17351150.

6. Phielix E, Schrauwen-Hinderling VB, Mensink M, Lenaers E, Meex R, Hoeks J, et al. Lower intrinsic ADP-stimulated mitochondrial respiration underlies in vivo mitochondrial dysfunction in muscle of male type 2 diabetic patients. Diabetes. 2008 Nov;57(11):2943-9. PubMed PMID: 18678616. Pubmed Central PMCID: 2570390.

7. Kelley DE, He J, Menshikova EV, Ritov VB. Dysfunction of mitochondria in human skeletal muscle in type 2 diabetes. Diabetes. 2002 Oct;51(10):2944-50. PubMed PMID: 12351431.

8. Kinugawa S, Takada S, Matsushima S, Okita K, Tsutsui H. Skeletal Muscle Abnormalities in Heart Failure. International heart journal. 2015;56(5):475-84. PubMed PMID: 26346520.

9. Lavine KJ, Sierra OL. Skeletal muscle inflammation and atrophy in heart failure. Heart failure reviews. 2017 Mar;22(2):179-89. PubMed PMID: 28091823. Pubmed Central PMCID: 5352459.

10. Konokhova Y, Spendiff S, Jagoe RT, Aare S, Kapchinsky S, MacMillan NJ, et al. Failed upregulation of TFAM protein and mitochondrial DNA in oxidatively deficient fibers of chronic obstructive pulmonary disease locomotor muscle. Skeletal muscle. 2016;6:10. PubMed PMID: 26893822. Pubmed Central PMCID: 4758107.

11. Remels AH, Schrauwen P, Broekhuizen R, Willems J, Kersten S, Gosker HR, et al. Peroxisome proliferator-activated receptor expression is reduced in skeletal muscle in COPD. The European respiratory journal. 2007 Aug;30(2):245-52. PubMed PMID: 17459894.

12. van den Borst B, Slot IG, Hellwig VA, Vosse BA, Kelders MC, Barreiro E, et al. Loss of quadriceps muscle oxidative phenotype and decreased endurance in patients with mild-to-moderate COPD. Journal of applied physiology. 2013 May;114(9):1319-28. PubMed PMID: 22815389. 
13. Mootha VK, Lindgren CM, Eriksson KF, Subramanian A, Sihag S, Lehar J, et al. PGC-1alpha-responsive genes involved in oxidative phosphorylation are coordinately downregulated in human diabetes. Nature genetics. $2003 \mathrm{Jul}$; 34(3):267-73. PubMed PMID: 12808457.

14. Patti ME, Butte AJ, Crunkhorn S, Cusi K, Berria R, Kashyap S, et al. Coordinated reduction of genes of oxidative metabolism in humans with insulin resistance and diabetes: Potential role of PGC1 and NRF1. Proceedings of the National Academy of Sciences of the United States of America. 2003 Jul 8;100(14):8466-71. PubMed PMID: 12832613. Pubmed Central PMCID: 166252.

15. Guo Y, Gosker HR, Schols AM, Kapchinsky S, Bourbeau J, Sandri M, et al. Autophagy in locomotor muscles of patients with chronic obstructive pulmonary disease. American journal of respiratory and critical care medicine. 2013 Dec 1;188(11): 1313-20. PubMed PMID: 24228729.

16. Kruse R, Vind BF, Petersson SJ, Kristensen JM, Hojlund K. Markers of autophagy are adapted to hyperglycaemia in skeletal muscle in type 2 diabetes. Diabetologia. 2015 Sep;58(9):2087-95. PubMed PMID: 26048236.

17. Ceelen JJM, Schols A, Kneppers AEM, Rosenbrand R, Drozdz MM, van Hoof SJ, et al. Altered protein turnover signaling and myogenesis during impaired recovery of inflammation-induced muscle atrophy in emphysematous mice. Scientific reports. 2018 Jul 17;8(1):10761. PubMed PMID: 30018383. Pubmed Central PMCID: 6050248.

18. Langen RC, Haegens A, Vernooy JH, Wouters EF, de Winther MP, Carlsen H, et al. NF-kappaB activation is required for the transition of pulmonary inflammation to muscle atrophy. American journal of respiratory cell and molecular biology. 2012 Sep;47(3):288-97. PubMed PMID: 22538866.

19. Rinaldi M, Maes K, De Vleeschauwer S, Thomas D, Verbeken EK, Decramer M, et al. Long-term nose-only cigarette smoke exposure induces emphysema and mild skeletal muscle dysfunction in mice. Disease models \& mechanisms. 2012 May;5(3):333-41. PubMed PMID: 22279084. Pubmed Central PMCID: 3339827.

20. Langen RC, Schols AM, Kelders MC, van der Velden JL, Wouters EF, JanssenHeininger YM. Muscle wasting and impaired muscle regeneration in a murine model of chronic pulmonary inflammation. American journal of respiratory cell and molecular biology. 2006 Dec;35(6):689-96. PubMed PMID: 16794259.

21. Ceelen JJ, Langen RC, Schols AM. Systemic inflammation in chronic obstructive pulmonary disease and lung cancer: common driver of pulmonary cachexia? Current opinion in supportive and palliative care. 2014 Dec;8(4):339-45. PubMed PMID: 25158627.

22. Sinden NJ, Stockley RA. Systemic inflammation and comorbidity in COPD: a result of 'overspill' of inflammatory mediators from the lungs? Review of the evidence. Thorax. 2010 Oct;65(10):930-6. PubMed PMID: 20627907.

23. van den Borst B, Gosker HR, Wesseling G, de Jager W, Hellwig VA, Snepvangers FJ, et al. Low-grade adipose tissue inflammation in patients with mild-to-moderate chronic obstructive pulmonary disease. The American journal of clinical nutrition. 2011 Dec;94(6):1504-12. PubMed PMID: 22071714.

24. Nishimura S, Manabe I, Nagai R. Adipose tissue inflammation in obesity and metabolic syndrome. Discovery medicine. 2009 Aug;8(41):55-60. PubMed PMID: 19788868. 
25. Mofarrahi M, Sigala I, Guo Y, Godin R, Davis EC, Petrof B, et al. Autophagy and skeletal muscles in sepsis. PloS one. 2012;7(10):e47265. PubMed PMID: 23056618. Pubmed Central PMCID: 3467208.

26. Piquereau J, Godin R, Deschenes S, Bessi VL, Mofarrahi M, Hussain SN, et al. Protective role of PARK2/Parkin in sepsis-induced cardiac contractile and mitochondrial dysfunction. Autophagy. 2013 Nov 1;9(11):1837-51. PubMed PMID: 24121678.

27. Tanaka K, Tanaka M, Takegaki J, Fujino H. Preventive effects of electrical stimulation on inflammation-induced muscle mitochondrial dysfunction. Acta histochemica. 2016 May 18. PubMed PMID: 27209425.

28. Gram M, Vigelso A, Yokota T, Hansen CN, Helge JW, Hey-Mogensen M, et al. Two weeks of one-leg immobilization decreases skeletal muscle respiratory capacity equally in young and elderly men. Experimental gerontology. 2014 Oct;58:269-78. PubMed PMID: 25193555.

29. Dirks ML, Wall BT, van de Valk B, Holloway TM, Holloway GP, Chabowski A, et al. One Week of Bed Rest Leads to Substantial Muscle Atrophy and Induces WholeBody Insulin Resistance in the Absence of Skeletal Muscle Lipid Accumulation. Diabetes. 2016 Oct;65(10):2862-75. PubMed PMID: 27358494.

30. Remels AH, Pansters NA, Gosker HR, Schols AM, Langen RC. Activation of alternative NF-kappaB signaling during recovery of disuse-induced loss of muscle oxidative phenotype. American journal of physiology Endocrinology and metabolism. 2014 Mar;306(6):E615-26. PubMed PMID: 24425759.

31. Liu J, Peng Y, Cui Z, Wu Z, Qian A, Shang P, et al. Depressed mitochondrial biogenesis and dynamic remodeling in mouse tibialis anterior and gastrocnemius induced by 4-week hindlimb unloading. IUBMB life. 2012 Nov;64(11):901-10. PubMed PMID: 23024034.

32. Cassano P, Sciancalepore AG, Pesce V, Fluck M, Hoppeler H, Calvani M, et al. Acetyl-L-carnitine feeding to unloaded rats triggers in soleus muscle the coordinated expression of genes involved in mitochondrial biogenesis. Biochimica et biophysica acta. 2006 Sep-Oct;1757(9-10):1421-8. PubMed PMID: 16814248.

33. Qi Z, Zhang Y, Guo W, Ji L, Ding S. Increased insulin sensitivity and distorted mitochondrial adaptations during muscle unloading. International journal of molecular sciences. 2012 Dec 11;13(12):16971-85. PubMed PMID: 23443131. Pubmed Central PMCID: 3546734.

34. Wagatsuma A, Kotake N, Kawachi T, Shiozuka M, Yamada S, Matsuda R. Mitochondrial adaptations in skeletal muscle to hindlimb unloading. Molecular and cellular biochemistry. 2011 Apr;350(1-2):1-11. PubMed PMID: 21165677.

35. Cannavino J, Brocca L, Sandri M, Bottinelli R, Pellegrino MA. PGC1-alpha overexpression prevents metabolic alterations and soleus muscle atrophy in hindlimb unloaded mice. The Journal of physiology. 2014 Oct 15;592(20):4575-89. PubMed PMID: 25128574. Pubmed Central PMCID: 4287741.

36. Kang C, Yeo D, Ji LL. Muscle Immobilization Activates Mitophagy and Disrupts Mitochondrial Dynamics in Mice. Acta physiologica. 2016 Apr 16. PubMed PMID: 27083499.

37. Vainshtein A, Desjardins EM, Armani A, Sandri M, Hood DA. PGC-1alpha modulates denervation-induced mitophagy in skeletal muscle. Skeletal muscle. 2015;5:9. PubMed PMID: 25834726. Pubmed Central PMCID: 4381453. 
38. Mazzatti DJ, Smith MA, Oita RC, Lim FL, White AJ, Reid MB. Muscle unloadinginduced metabolic remodeling is associated with acute alterations in PPARdelta and UCP-3 expression. Physiological genomics. 2008 Jul 15;34(2):149-61. PubMed PMID: 18445701.

39. Cannavino J, Brocca L, Sandri M, Grassi B, Bottinelli R, Pellegrino MA. The role of alterations in mitochondrial dynamics and PGC-1alpha over-expression in fast muscle atrophy following hindlimb unloading. The Journal of physiology. 2015 Apr 15; 593(8):1981-95. PubMed PMID: 25565653. Pubmed Central PMCID: 4405755.

40. Furuya N, Ikeda S, Sato S, Soma S, Ezaki J, Oliva Trejo JA, et al. PARK2/Parkinmediated mitochondrial clearance contributes to proteasome activation during slow-twitch muscle atrophy via NFE2L1 nuclear translocation. Autophagy. 2014 Apr;10(4):631-41. PubMed PMID: 24451648. Pubmed Central PMCID: 4091150.

41. Barberan-Garcia A, Rodriguez DA, Blanco I, Gea J, Torralba Y, Arbillaga-Etxarri A, et al. Non-anaemic iron deficiency impairs response to pulmonary rehabilitation in COPD. Respirology. 2015 Oct;20(7):1089-95. PubMed PMID: 26148453.

42. Robalo Nunes A, Tata M. The impact of anaemia and iron deficiency in chronic obstructive pulmonary disease: A clinical overview. Revista portuguesa de pneumologia. 2017 May - Jun;23(3):146-55. PubMed PMID: 28233650.

43. Jankowska EA, Rozentryt P, Witkowska A, Nowak J, Hartmann O, Ponikowska B, et al. Iron deficiency predicts impaired exercise capacity in patients with systolic chronic heart failure. Journal of cardiac failure. 2011 Nov;17(11):899-906. PubMed PMID: 22041326.

44. Stugiewicz M, Tkaczyszyn M, Kasztura M, Banasiak W, Ponikowski P, Jankowska EA. The influence of iron deficiency on the functioning of skeletal muscles: experimental evidence and clinical implications. European journal of heart failure. 2016 Jan 21. PubMed PMID: 26800032.

45. Dziegala M, Josiak K, Kasztura M, Kobak K, von Haehling S, Banasiak W, et al. Iron deficiency as energetic insult to skeletal muscle in chronic diseases. Journal of cachexia, sarcopenia and muscle. 2018 Sep 4. PubMed PMID: 30178922.

46. Allen GF, Toth R, James J, Ganley IG. Loss of iron triggers PINK1/Parkin-independent mitophagy. EMBO reports. 2013 Dec;14(12):1127-35. PubMed PMID: 24176932.

47. Roca M, Verduri A, Corbetta L, Clini E, Fabbri LM, Beghe B. Mechanisms of acute exacerbation of respiratory symptoms in chronic obstructive pulmonary disease. European journal of clinical investigation. 2013 May;43(5):510-21. PubMed PMID: 23489139.

48. Zhou X, Li Q, Zhou X. Exacerbation of Chronic Obstructive Pulmonary Disease. Cell biochemistry and biophysics. 2015 Nov;73(2):349-55. PubMed PMID: 27352322.

49. Dumitru L, Iliescu A, Dinu H, Badea R, Savulescu S, Huidu S, et al. Disability in COPD and Chronic Heart Failure Is the Skeletal Muscle the Final Common Pathway? Maedica. 2013 Jun;8(2):206-13. PubMed PMID: 24371487. Pubmed Central PMCID: 3865132.

50. Wei M, Gibbons LW, Kampert JB, Nichaman MZ, Blair SN. Low cardiorespiratory fitness and physical inactivity as predictors of mortality in men with type 2 diabetes. Annals of internal medicine. 2000 Apr 18;132(8):605-11. PubMed PMID: 10766678. 
51. Nickol AH, Frise MC, Cheng HY, McGahey A, McFadyen BM, Harris-Wright T, et al. A cross-sectional study of the prevalence and associations of iron deficiency in a cohort of patients with chronic obstructive pulmonary disease. BMJ open. 2015 Jul 6;5(7):e007911. PubMed PMID: 26150144. Pubmed Central PMCID: 4499677.

52. Silverberg DS, Mor R, Weu MT, Schwartz D, Schwartz IF, Chernin G. Anemia and iron deficiency in COPD patients: prevalence and the effects of correction of the anemia with erythropoiesis stimulating agents and intravenous iron. BMC pulmonary medicine. 2014;14:24. PubMed PMID: 24564844. Pubmed Central PMCID: 3946070.

53. Nairz M, Theurl I, Wolf D, Weiss G. Iron deficiency or anemia of inflammation? Differential diagnosis and mechanisms of anemia of inflammation. Wiener medizinische Wochenschrift. 2016 Oct;166(13-14):411-23. PubMed PMID: 27557596. Pubmed Central PMCID: 5065583.

54. Lainscak M, Gosker HR, Schols AM. Chronic obstructive pulmonary disease patient journey: hospitalizations as window of opportunity for extra-pulmonary intervention. Current opinion in clinical nutrition and metabolic care. 2013 May;16(3):278-83. PubMed PMID: 23507875.

55. Pedersen BK, Febbraio MA. Muscles, exercise and obesity: skeletal muscle as a secretory organ. Nature reviews Endocrinology. 2012 Apr 3;8(8):457-65. PubMed PMID: 22473333.

56. Forterre A, Jalabert A, Berger E, Baudet M, Chikh K, Errazuriz E, et al. Proteomic analysis of $\mathrm{C} 2 \mathrm{C} 12$ myoblast and myotube exosome-like vesicles: a new paradigm for myoblast-myotube cross talk? PloS one. 2014;9(1):e84153. PubMed PMID: 24392111. Pubmed Central PMCID: 3879278.

57. Kim S, Lee MJ, Choi JY, Park DH, Kwak HB, Moon S, et al. Roles of Exosome-Like Vesicles Released from Inflammatory C2C12 Myotubes: Regulation of Myocyte Differentiation and Myokine Expression. Cellular physiology and biochemistry: international journal of experimental cellular physiology, biochemistry, and pharmacology. 2018;48(5):1829-42. PubMed PMID: 30092568.

58. Lauritzen HP, Brandauer J, Schjerling P, Koh HJ, Treebak JT, Hirshman MF, et al. Contraction and AICAR stimulate IL- 6 vesicle depletion from skeletal muscle fibers in vivo. Diabetes. 2013 Sep;62(9):3081-92. PubMed PMID: 23761105. Pubmed Central PMCID: 3749330.

59. Phinney DG, Di Giuseppe M, Njah J, Sala E, Shiva S, St Croix CM, et al. Mesenchymal stem cells use extracellular vesicles to outsource mitophagy and shuttle microRNAs. Nature communications. 2015 Oct 7;6:8472. PubMed PMID: 26442449. Pubmed Central PMCID: 4598952.

60. Davis CH, Kim KY, Bushong EA, Mills EA, Boassa D, Shih T, et al. Transcellular degradation of axonal mitochondria. Proceedings of the National Academy of Sciences of the United States of America. 2014 Jul 1;111(26):9633-8. PubMed PMID: 24979790. Pubmed Central PMCID: 4084443.

61. Falchi AM, Sogos V, Saba F, Piras M, Congiu T, Piludu M. Astrocytes shed large membrane vesicles that contain mitochondria, lipid droplets and ATP. Histochemistry and cell biology. 2013 Feb;139(2):221-31. PubMed PMID: 23108569. 
62. Klionsky DJ, Abdelmohsen K, Abe A, Abedin MJ, Abeliovich H, Acevedo Arozena $A$, et al. Guidelines for the use and interpretation of assays for monitoring autophagy (3rd edition). Autophagy. 2016;12(1):1-222. PubMed PMID: 26799652. Pubmed Central PMCID: 4835977.

63. Kimura T, Takabatake Y, Takahashi A, Isaka Y. Chloroquine in cancer therapy: a double-edged sword of autophagy. Cancer research. 2013 Jan 1;73(1):3-7. PubMed PMID: 23288916.

64. Rodger CE, McWilliams TG, Ganley IG. Mammalian mitophagy - from in vitro molecules to in vivo models. The FEBS journal. 2018 Apr;285(7):1185-202. PubMed PMID: 29151277. Pubmed Central PMCID: 5947125.

65. Romanello V, Sandri M. Mitochondrial Quality Control and Muscle Mass Maintenance. Frontiers in physiology. 2015;6:422. PubMed PMID: 26793123. Pubmed Central PMCID: 4709858.

66. Lemasters JJ. Selective mitochondrial autophagy, or mitophagy, as a targeted defense against oxidative stress, mitochondrial dysfunction, and aging. Rejuvenation research. 2005 Spring;8(1):3-5. PubMed PMID: 15798367.

67. Zhang W, Siraj S, Zhang R, Chen Q. Mitophagy receptor FUNDC1 regulates mitochondrial homeostasis and protects the heart from I/R injury. Autophagy. 2017 Jun 3;13(6):1080-1. PubMed PMID: 28323531. Pubmed Central PMCID: 5486361.

68. Zhang T, Xue L, Li L, Tang C, Wan Z, Wang R, et al. BNIP3 Protein Suppresses PINK1 Kinase Proteolytic Cleavage to Promote Mitophagy. The Journal of biological chemistry. 2016 Oct 07;291(41):21616-29. PubMed PMID: 27528605. Pubmed Central PMCID: 5076832.

69. Kim Y, Triolo M, Hood DA. Impact of Aging and Exercise on Mitochondrial Quality Control in Skeletal Muscle. Oxidative medicine and cellular longevity. 2017;2017:3165396. PubMed PMID: 28656072. Pubmed Central PMCID: 5471566.

70. Drake JC, Wilson RJ, Yan Z. Molecular mechanisms for mitochondrial adaptation to exercise training in skeletal muscle. FASEB journal : official publication of the Federation of American Societies for Experimental Biology. 2016 Jan;30(1):13-22. PubMed PMID: 26370848.

71. Kneppers AEM. Unbiased analysis of skeletal muscle molecular responses upon pulmonary rehabilitation in advanced COPD. (Abstract) Week van de longen. 2018 10-04-2018. Epub 10-04-2018.

72. Puente-Maestu L, Perez-Parra J, Godoy R, Moreno N, Tejedor A, GonzalezAragoneses $\mathrm{F}$, et al. Abnormal mitochondrial function in locomotor and respiratory muscles of COPD patients. The European respiratory journal. 2009 May;33(5): 1045-52. PubMed PMID: 19129279.

73. Powers SK, Wiggs MP, Duarte JA, Zergeroglu AM, Demirel HA. Mitochondrial signaling contributes to disuse muscle atrophy. American journal of physiology Endocrinology and metabolism. 2012 Jul 1;303(1):E31-9. PubMed PMID: 22395111. Pubmed Central PMCID: 3404565.

74. Ko F, Abadir P, Marx R, Westbrook R, Cooke C, Yang H, et al. Impaired mitochondrial degradation by autophagy in the skeletal muscle of the aged female interleukin 10 null mouse. Experimental gerontology. 2016 Jan;73:23-7. PubMed PMID: 26596403. Pubmed Central PMCID: 4725733. 
Summary 


\section{Summary}

Skeletal muscle weakness is common in Chronic Obstructive Pulmonary Disease (COPD) and contributes to increased morbidity, mortality, and decreased health status. In addition to the loss of muscle mass, loss of skeletal muscle oxidative capacity is a well-established driver of skeletal muscle weakness in COPD patients. Skeletal muscle oxidative capacity is mainly determined by mitochondrial quantity, which is regulated by the balance between mitochondrial biogenesis and mitochondrial clearance. The latter can be achieved via several routes, including mitophagy and via secretion of mitochondria-containing extracellular vesicles. Although mitochondrial biogenesis has been studied extensively in the skeletal muscle of COPD patients, little is known about mitochondrial clearance. The general aim of this thesis was to investigate if COPD-related manifestations including muscle disuse, systemic inflammation, and more speculatively, iron deficiency, result in the onset of mitochondrial clearance, and consequently in the loss of muscle oxidative capacity.

Chapter 2 describes a literature review in which several extra-pulmonary manifestations of COPD, i.e. muscle disuse, systemic inflammation, hypoxia, and iron deficiency, which have previously been associated with the loss of skeletal muscle oxidative capacity, are described as possible triggers for skeletal muscle mitophagy. As some of these manifestations are also present in congestive heart failure (CHF) and type 2 diabetes (T2D), a comparable regulation of mitophagyinduced loss of muscle oxidative capacity in these disorders was proposed. Moreover, it was noted that mitophagy is a mechanism to degrade dysfunctional mitochondria in physiological conditions, and that inhibition of mitophagy via pharmacological intervention might result in increased mitochondrial damage and aggravation of muscle pathology.

In chapter 3, we executed a cross-sectional study investigating if altered molecular mitochondrial breakdown signalling is present in COPD patients. We reported molecular signalling patterns indicative of increased mitochondrial breakdown in skeletal muscle of patients with COPD, which were characterized by loss of muscle oxidative capacity. Furthermore, iron deficient patients had lower BNIP3L protein and higher BNIP3 mRNA expression levels, while patients with high circulating CRP levels had higher BNIP3 and autophagy-related protein levels. These data suggest that mitochondrial breakdown is increased in patients with COPD, and underlines a possible role for systemic inflammation as a mitochondrial breakdown-inducing manifestation. 
In chapter 4, we used a murine model of pulmonary-induced systemic inflammation, to study if systemic inflammation is able to result in increased mitophagy- and decreased mitochondrial biogenesis signalling patterns in an isolated setting. Mice were subjected to an intra-tracheal instillation of LPS, and sacrificed $24,48,72,96$, or $120 \mathrm{~h}$ later. Clear signalling patterns indicating a temporal increase in mitophagy and decrease in mitochondrial biogenesis were found in the m. gastrocnemius of these mice, without an apparent loss of mitochondrial quantity. These data suggest that one bolus of intra-tracheal LPS instillation results in the temporal modulation of mitochondrial clearance and biogenesis signalling, but is insufficient to result in reductions in mitochondrial content.

To study if short-term muscle disuse results in increased mitophagy- and decreased mitochondrial biogenesis signalling in an isolated setting, we conducted a combined analysis in a murine and a human study model in chapter 5. Mice were subjected to 3 days of skeletal muscle unloading via hind-limb suspension and human participants were subjected to 7 days of leg casting. Both in mice ( $m$. gastrocnemius) and men ( $m$. vastus lateralis) short-term skeletal muscle unloading resulted in molecular marker patterns indicative of increased receptor-mediated mitophagy and decreased transcriptional regulation of mitochondrial biogenesis, before the loss of mitochondrial content. These results emphasize the early-onset of skeletal muscle disuse-induced mitochondrial remodelling.

To test if mitophagy signalling could also be part of physiological adaptation during recovery from disuse-induced muscle pathology, chapter 6 first describes a cluster analysis of molecular markers of several mass- and metabolic plasticity-related processes from human participants subjected to 7 days of muscle reloading after 7 days of skeletal muscle casting. This cluster analysis was complemented with a murine time-course study with 1, 2, 3, 5, and 8 days of reloading after 14 days of hind-limb suspension. This chapter describes that the molecular regulation of protein turnover, mitochondrial turnover, and myonuclear turnover are correlated and temporally associated during recovery from atrophy, which suggests an interdependency or joint regulation of these muscle mass- and oxidative metabolism-related processes during recovery from muscle disuse.

In chapter 7, we conducted an in vitro study to determine if iron chelation results in the decrease of skeletal muscle mitochondrial content and function, and if this decrease is caused by increased mitochondrial clearance via mitophagy. We 
show that both mitochondrial-lysosomal localisation, indicative of mitophagy, and secretion of mitochondrial-containing extracellular vesicles (EV) are present in iron chelation-exposed myotubes. Moreover, we show that mitophagy is not essential for development of iron chelation-induced mitochondrial impairments. Together, these results suggest a possible interplay between different pathways of mitochondrial clearance, which may contribute to the mitochondrial impairments observed upon iron chelation.

In chapter 8, we discuss and conclude that skeletal muscle mitophagyregulation is increased in COPD based on current evidence. Whether this is also true for other chronic disorders such as T2D and CHF requires further investigation. Furthermore, we established that systemic inflammation, muscle disuse, recovery after muscle disuse, and iron deficiency are all able to alter mitochondrial clearance regulation in skeletal muscle, but chronic versus acute exposure to these triggers needs to be studied in more detail. Interestingly, as skeletal muscle is able to achieve mitochondrial clearance via secretion of mitochondria via EV, this raises the question if muscle cells are also able to integrate functional mitochondria from EV in their mitochondrial network, providing new possibilities for future oxidative capacity preserving or boosting therapies. Since mitochondrial breakdown is an important part of the skeletal muscle recovery process, interfering with mitochondrial clearance itself as oxidative capacity preserving therapy may not be ideal. Future research should therefore also aim to determine whether mitochondrial clearance-induced loss of oxidative capacity can be prevented or restored using lifestyle modifications as well as nutritional or physical interventions. 
Nederlandse samenvatting 
COPD, de afkorting voor de Engelstalige term Chronic Obstructive Pulmonary Disease, is een veelvoorkomende chronische ziekte waarbij de longen onherstelbaar beschadigd zijn. COPD patiënten hebben niet alleen moeilijkheden met ademen maar ook de skeletspieren in bijvoorbeeld de armen en benen van deze patiënten zijn verzwakt. Spierzwakte verergert de ziektelast en wordt veroorzaakt door verlies van spiermassa en door verlies van oxidatieve capaciteit, het vermogen van de spier om zuurstof te gebruiken. Deze oxidatieve capaciteit wordt voornamelijk bepaald door de hoeveelheid mitochondriën in de spier.

\begin{abstract}
“Mitochondriën zijn organellen welke de 'energiefabriekjes' van de cel zijn. In de mitochondriën worden suikers en vetten met behulp van zuurstof omgezet in pakketjes met energie (ATP). Dit ATP kan door de cel gebruikt worden voor allerlei processen, zoals het samentrekken van de spieren. Zonder mitochondriën kan er wel ATP worden geproduceerd, maar dit gaat veel minder efficiënt."
\end{abstract}

De aanmaak (biogenese) en afbraak van mitochondriën worden nauwkeurig gereguleerd voor het in stand houden van de totale hoeveelheid mitochondriën in de spier. Tot op heden is alleen de mitochondriële biogenese uitgebreid onderzocht in de skeletspier van COPD patiënten. Er is daarentegen maar weinig bekend over de regulatie van de mitochondriële afbraak of andere processen die bij verlies van mitochondriën betrokken zijn. Mitochondriën kunnen in de cel worden afgebroken door verschillende processen waarvan mitofagie de belangrijkste is. Mitochondriën kunnen ook door de cel worden uitgescheiden via zogenaamde vesikels.

\footnotetext{
"Mitofagie is een selectieve vorm van autofagie waarbij specifiek mitochondriën worden afgebroken. Tijdens autofagie wordt een autofagosomaal membraan rondom een bepaald stuk cel of organel aangelegd, wat deze isoleert van de rest van de cel. Dit wordt het autofagosoom genoemd. Vervolgens wordt het autofagosoom samengevoegd met een lysosoom, waarin zich eiwitten bevinden die gespecialiseerd zijn in het afbreken van organellen en andere eiwitten. Hier wordt vervolgens de inhoud van het autofagosoom afgebroken. Autofagie wordt mitofagie genoemd als specifiek mitochondriën worden opgenomen en afgebroken in deze autofagosomen en lysosomen. Behalve een autofagosomaal membraan kunnen mitochondriën ook opgenomen worden in vesikels. Tijdens dit proces wordt het te verwijderen mitochondrion omgeven door een membraan en wordt dit membraan met mitochondrion erin volledig door de cel uitgescheiden."
} 
De overkoepelende hypothese van de studies beschreven in deze dissertatie is dat diverse COPD-gerelateerde manifestaties zoals verminderd spiergebruik door lichamelijke inactiviteit, systemische ontsteking, en mogelijk ook ijzertekort, leiden tot initiatie van mitochondriële afbraak en/of uitscheiding via vesikels, en daarmee tot het verlies van oxidatieve capaciteit in de spier.

In hoofdstuk 2 wordt een literatuurstudie beschreven waarin we eerst de huidige studies naar mitochondriële afbraak in de skeletspier van de chronische ziektes COPD, Diabetes type 2 (T2D) en hartfalen (CHF) beschrijven. Vervolgens analyseren we wat het huidige bewijs is dat manifestaties die veel voorkomen bij deze ziektes, zoals verminderd spiergebruik, systemische ontsteking, zuurstoftekort en ijzertekort, kunnen aanzetten tot mitochondriële afbraak. We concluderen dat het waarschijnlijk is dat de aanwezigheid van deze manifestaties bij COPD patiënten kunnen leiden tot een verhoogde mitochondriële afbraak. Verder benadrukken we dat de mitochondriële afbraak een belangrijk proces is voor behoud van een gezonde mitochondriële populatie, en dat het remmen van mitochondriële afbraak dus mogelijk niet alleen tot een verhoogde mitochondriële hoeveelheid zal leiden, maar wellicht ook nadelig kan zijn wanneer beschadigde mitochondriën zich ophopen.

Voor hoofdstuk 3 is een cross-sectionele studie uitgevoerd waarin we de moleculaire signalering die mitochondriële afbraak aanstuurt in COPD patiënten en gezonde controles hebben onderzocht. We beschrijven de aanwezigheid van signaleringspatronen die indicatief zijn voor mitochondriële afbraak in de skeletspier van COPD patiënten waarbij de oxidatieve capaciteit in de spier verlaagd is. Verder laten we zien dat patiënten met een ijzertekort lagere BNIP3L eiwit en hogere BNIP3 genexpressie hebben, en dat patiënten met hoge systemische ontstekingswaarden hoge BNIP3 en autofagie-gerelateerde eiwit expressie hebben. Gecombineerd maken deze gegevens het aannemelijk dat mitochondriële afbraak verhoogd is in COPD patiënten vergeleken met gezonde controles, en dat systemische ontsteking de mitochondriële afbraak kan verhogen.

"BNIP3 en BNIP3L zijn eiwitten die op mitochondriën zitten en verantwoordelijk zijn voor het rekruteren van autofagosomale membranen tijdens mitofagie. De hoeveelheden van deze eiwitten zijn sterk afhankelijk van de condities waarin de spiercellen zich bevinden en de hoeveelheid eiwitten op mitochondriën kan zeer snel groter of kleiner worden als de spier zich moet aanpassen." 
Om te kijken of systemische ontsteking tot verhoogde signalering van mitochondriële afbraak en verlaagde signalering van mitochondriële aanmaak kan leiden, hebben we in hoofdstuk 4 muizen blootgesteld aan een injectie met LPS of $\mathrm{NaCl}$ in de longen en skeletspieren uit de achterpoot geïsoleerd op $24,48,72,96$, en 120 u na de blootstelling. We vonden signaleringspatronen die indicatief zijn voor tijdelijk verhoogde mitofagie en verlaagde mitochondriële biogenese, zonder dat dit leidde tot een verlies in mitochondriële massa. Deze resultaten maken het aannemelijk dat één acute LPS-geïnduceerde luchtwegontsteking voldoende is om tijdelijke verhoging in signalering van mitofagie en verlaging van signalering van mitochondriële aanmaak te bewerkstellingen, maar waarschijnlijk onvoldoende is om uiteindelijk te resulteren in een verlies van mitochondriële hoeveelheid.

"Intra-tracheale injectie met LPS (IT-LPS) wordt toegepast in een muizenmodel waar een ontsteking wordt gemodelleerd in de longen van muizen door een bacteriële component (genaamd LPS) via de luchtpijp (trachea) in de longen te injecteren. De longontsteking die hierop volgt bereidt zich vervolgens uit naar het bloed waardoor de ontsteking in het gehele lichaam, en dus ook bij de spieren, terecht komt."

Vervolgens hebben we in hoofdstuk 5 gekeken of het kortdurend niet gebruiken van skeletspieren genoeg is om te zorgen voor een verhoogde mitochondriële afbraak en een verlaagde mitochondriële aanmaak signalering in zowel een muizenstudie als een humane studie. We maakten het de muizen onmogelijk om gedurende drie dagen hun spieren in hun achterpoot te belasten, door deze muizen in een harnas te hangen zodat ze alleen met de voorpoten en niet met de achterpoten op de grond konden komen. Bij gezonde vrijwilligers is één been één week lang ingegipst en werd gevraagd om dit been zo veel mogelijk te ontlasten. In dit hoofdstuk laten we zien dat zowel in de spieren van de muizen als ook in de spieren van de mensen het kortdurende verminderd belasten van de spier leidde tot signaleringspatronen welke indicatief zijn voor verhoogde mitofagie en verlaagde regulatie van mitochondriële aanmaak, nog vóór het verlies van mitochondriële hoeveelheid meetbaar is. Deze resultaten tonen aan dat als spieren niet belast worden, processen van mitochondriële regulatie snel veranderen ten nadele van de oxidatieve capaciteit.

In hoofdstuk 6 hebben we onderzocht of mitofagie-signalering ook onderdeel is van de herstelrespons van de spier na een periode van verminderde belasting van de spier. Om dit te bewerkstelligen hebben we veranderingen in verschillende processen vergeleken in de beenspier van gezonde vrijwilligers 
gedurende een één-week-durende herstelperiode na één week van verminderde spierbelasting door ingipsen. Deze analyse hebben we gecombineerd met analyses voor dezelfde signalering in een muizenstudie waarin muizen twee weken hun achterpoot niet konden belasten, gevolgd door een herstelperiode van 1, 2, 3, 5, of 8 dagen. In dit hoofdstuk beschrijven we dat de moleculaire regulatie van spiermassa-herstel geassocieerd is met de moleculaire regulatie van mitochondriële afbraak. Ook laten we zien dat de moleculaire regulatie van mitochondriële afbraak op verschillende momenten gedurende de herstelfase tijdelijk veranderd is. Deze resultaten maken het aannemelijk dat de processen van spiermassa-groei, spiermassaafbraak en mitochondriële afbraak van elkaar afhankelijk zijn en dat zij samen voor het spierherstel zorgen tijdens de herstelfase na verminderde belasting van de spier.

In hoofdstuk 7 beschrijven we een in vitro studie waarin we hebben bepaald of ijzertekort leidt tot het verlies van mitochondriële massa en functie in spiercellen en of dit verlies wordt veroorzaakt door mitochondriële afbraak via mitofagie. We laten zien dat mitochondriën aanwezig zijn in lysosomale structuren in de cellen die blootgesteld waren aan ijzertekort, wat duidt op meer mitofagie. Deze mitofagie bleek echter niet essentieel te zijn voor het verlies van mitochondriële massa en functie, waaruit we concludeerde dat er nog een ander mechanisme van mitochondrieel verlies geactiveerd moest zijn. Inderdaad deden we een erg interessante en vernieuwende observatie dat mitochondriën aanwezig waren in vesikels die waren uitgescheiden door aan ijzertekort blootgestelde spiercellen, wat een mogelijk alternatief mechanisme is voor het verlies van mitochondriën. Gecombineerd maken deze resultaten het aannemelijk dat ijzertekort leidt tot een dynamisch samenspel van verschillende mechanismen die leiden tot een verlies in mitochondriële hoeveelheid.

In hoofdstuk 8 bediscussiëren en concluderen we dat de huidige beschikbare wetenschappelijke studies het aannemelijk maken dat mitofagie-signalering verhoogd is in de skeletspieren van COPD patiënten, maar dat de wetenschappelijke onderbouwing hiervoor in patiënten met hartfalen en diabetes type 2 nog niet toereikend is. Verder hebben we het aannemelijk gemaakt dat systemische ontsteking, verminderde spierbelasting, en ijzertekort allen kunnen leiden tot verhoogde mitochondriële afbraak en verlies. Mogelijke verschillen tussen chronische en acute blootstelling aan deze triggers dient echter nog verder onderzocht te worden. Erg interessant is de observatie dat spiercellen mitochondriën via vesikels kunnen uitscheiden, wat leidt tot de nieuwe onderzoeksvraag of mitochondriën via deze vesikels ook intact kunnen 
worden opgenomen door spiercellen. Deze onderzoekslijn zou tot onderzoek naar nieuwe therapieën kunnen leiden voor behoud van de oxidatieve capaciteit. Omdat mitochondriële afbraak een essentieel onderdeel is van het herstelproces na spierschade, is het remmen van mitochondriële afbraak ten behoeve van een verhoogde mitochondriële massa niet ideaal. Toekomstig onderzoek zal zich daarom ook moeten bezighouden met de vraag of de initiatie van mitochondriële afbraak kan worden voorkomen met behulp van veranderingen in de levensstijl van patiënten, of het aanpassen van voeding of fysieke training voor patiënten. 
Valorisation 


\section{Societal relevance}

Chronic obstructive pulmonary disease (COPD) is a lung disease characterized by irreversible airflow limitation in the lungs, and is currently the third leading cause of death in the world (1). Currently, there are almost 600.000 COPD patients in the Netherlands alone (2), and over 250 million patients worldwide (3). Although COPD is primarily a lung disease, patients often suffer from skeletal muscle dysfunction of which the two major underlying impairments include muscle wasting and the loss of mitochondrial quantity. These impairments may also contribute to the development of co-morbidities such as diabetes or cardiovascular disease (4). Extrapulmonary impairments and comorbidities contribute to an increased mortality, decreased quality of life, and increased healthcare costs (4-6). Prevention or reversion of skeletal muscle impairments can therefore contribute to increasing quality of life and decreasing COPD-related healthcare costs.

The loss of skeletal muscle mitochondrial quantity in COPD patients suggests that these patients have an altered balance between mitochondrial biogenesis and mitochondrial clearance. The role of impaired mitochondrial biogenesis regulation has been reported in several previous studies (7-9), but mitochondrial clearance has hardly been studied in muscle of COPD patients to date. Therefore, research aimed to unravel the role of skeletal muscle mitochondrial clearance is essential to understand the development of mitochondrial impairments in skeletal muscle of these patients (10).

In this thesis, we studied the regulation of mitochondrial clearance in skeletal muscle of COPD patients. Moreover, we applied in vivo and in vitro experimental models to study the effect of several isolated COPD-related factors that may affect skeletal muscle oxidative capacity $(11,12)$. Our results show that molecular signalling indicative of mitochondrial clearance is present in skeletal muscle of COPD patients who suffer from loss of muscle oxidative capacity. Moreover, systemic inflammation, muscle disuse, recovery of muscle disuse, and iron deficiency can individually contribute to the molecular regulation and dysregulation of skeletal muscle mitochondrial clearance. As other chronic diseases (e.g. congestive heart failure and type 2 diabetes) are also characterised by at least some of these factors, and suffer from skeletal muscle mitochondrial impairments, the work presented in this thesis may translate to these diseases as well. Therefore, we identified mitochondrial clearance as a potential therapeutic target in disorders characterized by loss of skeletal muscle mitochondrial capacity. Furthermore, as prevention of mitochondrial 
dysfunction could be preferred over the inhibition of mitochondrial clearance per se, we propose that lifestyle modifications, as well as nutritional or physical interventions targeting the upstream effectors (such as systemic inflammation, muscle disuse, or iron status) could prove beneficial as mitochondrial-clearance limiting and mitochondrial content-preserving therapies for a broad range of patients.

\section{Non-scientific audience}

As the presence of many of the above-mentioned factors, i.e. muscle disuse and iron deficiency, can be prevented by a healthy lifestyle, it is essential that we increase patients' and healthcare professionals awareness of their benefits. For example, skeletal muscle mitochondrial health greatly benefits from physical activity and a healthy diet. Moreover, since loss of skeletal muscle oxidative capacity already presents itself in mild-to-moderate COPD patients (9), the prevention of loss of oxidative capacity should already start in subjects at high risk for- or in an early stage of COPD.

\section{Innovation}

As stated above, several previous studies have reported changes in regulation of mitochondrial biogenesis in skeletal muscle of patients with COPD, and similar results were reported for patients with congestive heart failure and type 2 diabetes as well $(7-9,13,14)$. Interestingly, our study investigating mitochondrial clearance in skeletal muscle of COPD patients, is one of the first to report a comprehensive set of protein and mRNA expression levels of markers for mitochondrial breakdown and autophagy in combination with measurements for markers of mitochondrial quantity $(10,15)$. Therefore, our current results underline the importance to study mitochondrial homeostasis in a comprehensive approach, targeting multiple mechanisms of mitochondrial quantity regulation, even in the absence of changes in mitochondrial quantity.

The identification of mitochondria in extracellular vesicles secreted by skeletal muscle, as described in chapter 7 , is likely to have a high impact on our view of skeletal muscle mitochondrial homeostasis. The metabolic phenotype of skeletal muscle has been a popular research topic for many years, and has been studied in relation to many different diseases. Our observation that mitochondria can be secreted by skeletal muscle cells provides a novel paradigm 
in skeletal muscle cell plasticity research where regulation of mitochondrial homeostasis is currently still regarded as a single-cell and intra-cellular process (16).

Our data describing secretion of mitochondria-containing extracellular vesicles from muscle cells, in combination with previous studies reporting that muscle cells are able to secrete extracellular vesicles and non-muscular cells are able to secrete mitochondria via extracellular vesicles (17-20), suggests that skeletal muscle mitochondrial clearance is regulated at the inter-cellular level, providing interesting new targets for future oxidative capacity preserving therapies. Moreover, our work opens the door to a highly relevant research question: "Are skeletal muscle cells able to reintegrate functioning mitochondria from their extracellular environment?". Although highly speculative, this line of research could provide several important improvements to skeletal muscle oxidative capacity boosting therapies. For example, it might be opportunistic to explore possibilities to boost mitochondrial biogenesis and mitochondrialcontaining vesicle secretion in non-skeletal muscle cells, which could subsequently be incorporated into skeletal muscle. In addition, it might eventually be possible to generate a therapy in which patients are treated with elsewhere created intact mitochondria-containing vesicles which are incorporated into skeletal muscles, thus improving their oxidative capacity, contributing to increased exercise capacity and quality of life.

Since the pathways of mitochondrial homeostasis are greatly preserved in different tissues, it is likely that the fundamental knowledge concerning the initiation of mitochondria-containing vesicular excretion could be applied to other research disciplines as well. Especially studies on metabolic active tissues (e.g. cardiac muscle, liver, or brain) might benefit from knowledge obtained in the current thesis.

In conclusion, this thesis provides new insights in the etiology of chronic disease-driven muscle mitochondrial impairments and the putative role of mitophagy and mitochondrial clearance via vesicles secretion herein. These insights do not directly lead to new treatments at this moment, but are potentially important in generation of novel therapies aimed at muscle mitochondrial impairments in COPD and other chronic diseases. 


\section{References}

1. WHO. Fact sheets: The top 10 causes of death 20182018 [Available from: http:// www.who.int/news-room/fact-sheets/detail/the-top-10-causes-of-death.

2. Volksgezondhiedszorg. Prevalentie en nieuwe gevallen van COPD 2018 [Available from: https://www.volksgezondheidenzorg.info/onderwerp/copd/cijfers-context/ huidige-situatie\#node-prevalentie-en-nieuwe-gevallen-van-copd.

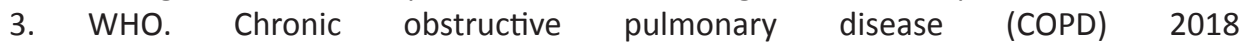
[Available from: http://www.who.int/news-room/fact-sheets/detail/ chronic-obstructive-pulmonary-disease-(copd).

4. Maltais F, Decramer M, Casaburi R, Barreiro E, Burelle $Y$, Debigare R, et al. An official American Thoracic Society/European Respiratory Society statement: update on limb muscle dysfunction in chronic obstructive pulmonary disease. American journal of respiratory and critical care medicine. 2014;189(9):e15-62.

5. Mannino DM, Higuchi K, Yu TC, Zhou H, Li Y, Tian H, et al. Economic Burden of COPD in the Presence of Comorbidities. Chest. 2015;148(1):138-50.

6. Jansson SA, Backman H, Ronmark E, Lundback B, Lindberg A. Hospitalization Due to Co-Morbid Conditions is the Main Cost Driver Among Subjects With COPD-A Report From the Population-Based OLIN COPD Study. Copd. 2015;12(4):381-9.

7. Konokhova Y, Spendiff S, Jagoe RT, Aare S, Kapchinsky S, MacMillan NJ, et al. Failed upregulation of TFAM protein and mitochondrial DNA in oxidatively deficient fibers of chronic obstructive pulmonary disease locomotor muscle. Skeletal muscle. 2016;6:10.

8. Remels AH, Schrauwen P, Broekhuizen R, Willems J, Kersten S, Gosker HR, et al. Peroxisome proliferator-activated receptor expression is reduced in skeletal muscle in COPD. The European respiratory journal. 2007;30(2):245-52.

9. van den Borst B, Slot IG, Hellwig VA, Vosse BA, Kelders MC, Barreiro E, et al. Loss of quadriceps muscle oxidative phenotype and decreased endurance in patients with mild-to-moderate COPD. Journal of applied physiology. 2013;114(9):1319-28.

10. Guo Y, Gosker HR, Schols AM, Kapchinsky S, Bourbeau J, Sandri M, et al. Autophagy in locomotor muscles of patients with chronic obstructive pulmonary disease. American journal of respiratory and critical care medicine. 2013;188(11):1313-20.

11. Gosker HR, Lencer NH, Franssen FM, van der Vusse GJ, Wouters EF, Schols AM. Striking similarities in systemic factors contributing to decreased exercise capacity in patients with severe chronic heart failure or COPD. Chest. 2003;123(5):1416-24.

12. Gosker HR, Wouters EF, van der Vusse GJ, Schols AM. Skeletal muscle dysfunction in chronic obstructive pulmonary disease and chronic heart failure: underlying mechanisms and therapy perspectives. The American journal of clinical nutrition. 2000;71(5):1033-47.

13. Mootha VK, Lindgren CM, Eriksson KF, Subramanian A, Sihag S, Lehar J, et al. PGC-1alpha-responsive genes involved in oxidative phosphorylation are coordinately downregulated in human diabetes. Naturegenetics. 2003;34(3):267-73.

14. Patti ME, Butte AJ, Crunkhorn S, Cusi K, Berria R, Kashyap S, et al. Coordinated reduction of genes of oxidative metabolism in humans with insulin resistance and diabetes: Potential role of PGC1 and NRF1. Proceedings of the National Academy of Sciences of the United States of America. 2003;100(14):8466-71. 
15. Kruse R, Vind BF, Petersson SJ, Kristensen JM, Hojlund K. Markers of autophagy are adapted to hyperglycaemia in skeletal muscle in type 2 diabetes. Diabetologia. 2015;58(9):2087-95.

16. Romanello V, Sandri M. Mitochondrial Quality Control and Muscle Mass Maintenance. Frontiers in physiology. 2015;6:422.

17. Phinney DG, Di Giuseppe M, Njah J, Sala E, Shiva S, St Croix CM, et al. Mesenchymal stem cells use extracellular vesicles to outsource mitophagy and shuttle microRNAs. Nature communications. 2015;6:8472.

18. Falchi AM, Sogos V, Saba F, Piras M, Congiu T, Piludu M. Astrocytes shed large membrane vesicles that contain mitochondria, lipid droplets and ATP. Histochemistry and cell biology. 2013;139(2):221-31.

19. Forterre A, Jalabert A, Berger E, Baudet M, Chikh K, Errazuriz E, et al. Proteomic analysis of $\mathrm{C} 2 \mathrm{C} 12$ myoblast and myotube exosome-like vesicles: a new paradigm for myoblast-myotube cross talk? PloS one. 2014;9(1):e84153.

20. Kim S, Lee MJ, Choi JY, Park DH, Kwak HB, Moon S, et al. Roles of Exosome-Like Vesicles Released from Inflammatory C2C12 Myotubes: Regulation of Myocyte Differentiation and Myokine Expression. Cellular physiology and biochemistry : international journal of experimental cellular physiology, biochemistry, and pharmacology. 2018;48(5):1829-42. 
Dankwoord 
In dit hoofdstuk wil ik graag iedereen bedanken die op de een of andere manier heeft bijgedragen aan de totstandkoming van dit proefschrift. Dit zijn veel meer mensen dan ik hier kan noemen, maar ik wil toch een aantal mensen in het bijzonder bedanken.

Allereerst wil ik mijn promotor Annemie bedanken. Ik waardeer alle kansen die je me hebt gegeven en de ruimte die ik kreeg om mijn eigen weg te gaan. Daarnaast heb ik ongelooflijk veel van je geleerd tijdens mijn PhD. Ik zal onze eerste ontmoeting op de tennisbaan - tijdens de Stennis/Ready mix - nooit vergeten, waarbij wij samen als team werden gekoppeld en een paar wedstrijden wisten te winnen. In die tijd was ik nog Bachelor student en het zou nog jaren duren voordat ik met mijn stage begon bij PUL. Tijdens die stage, tijdens welke wij elkaar wederom op de tennisbaan tegenkwamen, werd het plan gesmeed om een beursaanvraag te schrijven en de rest is geschiedenis. Helaas hebben we na de derby tussen de professoren (jij en Frederik-Jan) en de studenten (ik en Dirk) niet vaak meer op de baan gestaan, maar ik heb goede hoop dat dit in de toekomst weer zal gebeuren.

Harry, ook jij hebt een gigantische bijdrage gehad in alles wat ik heb geleerd en bereikt. Zonder jouw input was mijn PhD proposal nooit geaccepteerd. Ik waardeer de nuchtere en to-the-point samenwerking die wij hebben gehad, en accepteer dat jij het af en toe zwaar moet hebben gehad om mij gemotiveerd te houden na de 16e IT-LPS-manuscript afwijzing. Daarnaast heb ik erg genoten van de vele BBQs en borrels in zowel jouw als mijn tuin, deze waren altijd welkom. $\mathrm{Nu}$ heeft er vijf jaar lang enige onenigheid tussen ons bestaan, en daarom denk ik dat het op de valreep nog even belangrijk is om in deze wetenschappelijke publicatie te vermelden dat een boterham uit twee sneeën brood bestaat, zodat we die discussie ook eindelijk af kunnen sluiten.

Alex, ook jij hebt een flinke bijdrage geleverd aan de kansen die ik heb gekregen en het werk wat ik heb gedaan. Niet alleen stond jij direct klaar om met zeer opbouwend en sterk commentaar de kwaliteit van mijn GP-proposal flink te verbeteren, maar hebben we in de jaren van mijn $\mathrm{PhD}$ veel samengewerkt. Ook ben ik ongelooflijk dankbaar voor de twee maanden durende samenwerking die we na mijn promotie hebben gehad waar hopelijk nog veel publicaties uit komen. Daarnaast waren de vele gedeelde bier/BBQ momenten ook bijzonder welkom.

Ramon, ook jou wil ik graag bedanken. Niet alleen voor de gezelligheid en natuurlijk jouw gastvrijheid (mede als oppasadres voor Bowie), maar ook voor het delen van jouw uitgebreide kennis. Met name de diermodellen die ik heb 
gebruikt zijn vrijwel allemaal door jou, of in samenwerking met jou, ontwikkeld, en ik ben hier erg dankbaar voor. Verder waardeer ik het dat ik altijd even tussendoor bij je kon binnenschieten als ik iets nodig had.

Wouter, allereerst bedankt dat je mijn paranimf wilt zijn. Het was me een genoegen om je te verwelkomen binnen onze afdeling en ik was verrast door de vele hobby's die we deelde. Jij was eigenlijk altijd wel in voor biertjes, whiskietjes, BBQs, festivals, of fitness. Daarnaast heb ik je nog weten te overtuigen om een mooie racefiets aan te schaffen en hebben we sindsdien vele en vele kilometers samen versleten (onder andere in Nederland, België, Duitsland, Italië, en misschien binnenkort wel Denemarken). Ik kijk uit naar onze toekomstige fietstochten, al kan ik je allang niet meer bijhouden. Oh, en voordat ik het vergeet, het chauffeuren van de Maserati op mijn bruiloft werd ook erg gewaardeerd. Jouw promotie is nog redelijk ver weg maar ik ben ervan overtuigd dat het met vlag-en-wimpel lukt.

Anita, jij bent zonder twijfel degene geweest waar ik het meest mee samen heb gewerkt. Zo zal ik nooit vergeten dat we bij het afsluiten van een vergadering om 19.00 u het verzoek kregen om een humane-spier-database even opnieuw te analyseren voor de vergadering van $08.30 \mathrm{~h}$ de volgende dag, en dat PonceauS eigenlijk maar een slechte staining is. Maar meer nog blijven mij de vakantie in de Ardeche, en de tripjes naar bijvoorbeeld London bij (waarbij je vriendelijk vroeg of ik niet jouw -meer dan dubieuze- cosmetica in mijn rugzak door de vliegtuigbeveiliging wilde loodsen, om vervolgens zelf aangehouden te worden door de beveiliging). Ik hoop dat jij en Roy weer snel bij elkaar zullen zijn, en dat jullie droom om op hetzelfde continent mooie wetenschap te bedrijven snel werkelijkheid wordt. Lyon, Toronto, London, London, Nimes, Maastricht of Aarhus, ik hoop dat we elkaar nog regelmatig zullen tegenkomen en dat er eventueel nog een vakantietje inzit.

Wessel, ik heb jou altijd een fijne en relaxte onderzoeker gevonden. Ik heb bij jou nog altijd het idee dat elk experiment gewoon de eerste keer lukt, maar dat kan ook komen omdat je niet veel klaagt en gewoon doorgaat. Ik hoop dat je ervoor kiest om in het onderzoek te blijven want je zou een aanwinst zijn. Ook nog bijzonder bedankt voor de spelletjesavonden (motto: al het goud is voor Wessel), de PUL-ritjes die we maakte (alleen bij mooi weer), en de (ginger)biertjes die we dronken. Rosanne, al zaten onze skills misschien wat verder van elkaar af, ben ook jij een onderzoeker waarvan ik $100 \%$ zeker weet dat je goed terecht komt. Ik wil niet eens weten hoeveel koffie wij samen hebben gedronken en ook wij hebben de nodige drankjes, BBQs, en borrels versleten (allemaal zeer welkom). Al heb je jouw prioriteiten nog steeds niet helemaal op orde (handbal vs. 
borrels), ik heb er vertrouwen in dat dit binnenkort goed komt. Sarah, bedankt voor de tijd die we samen op de kamer zaten, ik hoop dat je een mooie toekomst tegemoet gaat. Chiel, jij bent zonder twijfel degene die mij het meest geleerd heeft in het lab. Ik ben erg dankbaar voor alle technische vaardigheden die ik van je kon overnemen, en heb in het bijzonder genoten van die blauwe maandag dat we regelmatig samen mountainbikete (met uitzondering van het incidentje waarbij ik vanuit stilstand zo een struik brandnetels in kieperde). Marco, ook jou ben ik erg dankbaar voor de ondersteuning die jij mij gaf in de afgelopen 5 jaar. Karin, wij zijn even lang lid geweest van de PUL-familie en het was leuk om te zien dat we vaak in dezelfde fases terecht kwamen. Zo liepen we tegelijkertijd stage, schreven we een proposal, proberen we onze artikelen te publiceren en als allerlaatst schreven we ons boekje. Ik hoop dat je gaat genieten van het arts-zijn. Mieke, jij was degene waar ik onderwijs-technisch het meeste van heb geleerd. Ik wil je graag bedanken voor alle hulp bij mijn BKO, en dat ik in een aantal van jouw blokken onderwijs heb mogen verzorgen. Niki, wij hebben vooral een uitgebreide labuitje-geschiedenis. Ofwel samen in de organisatie, of samen in een team. Ik wil je bedanken voor alle strijd die we hebben mogen leveren tijdens deze dagen. Sara, Martijn, Lisanne, Bregtje, Jules, Juliette, Rianne, Caspar, Cheryl, Juanita, Viviënne, Coby, \& Vasili, ook jullie bedankt voor de gezelligheid tijdens de lunch, sinterklaasborrels, kerstborrels, labuitjes en ga zo maar even door. Ik waardeer het erg dat pulmonologie een hechte groep is, en hoop dat dit nog lang zo zal zijn.

Verder wil ik Kasper \& Marijke in het bijzonder bedanken voor de gastvrijheid en de kennis van de autophagy groep, en het altijd mogen lenen van antilichamen, vesikelisolatiedevices ed. Ook Lex \& Luc heel erg bedankt voor de prettige samenwerking binnen de unloading en reloading studies. In addition, Mitja, thank you for the nice collaboration and the acces to all the biopsies.

Buiten het werk zijn ook mijn vrienden en familie erg belangrijk voor mij geweest. Zonder jullie is het leven een stuk minder leuk en ik ben jullie daarom erg dankbaar dat jullie er voor me zijn. Ook hiervan wil ik een aantal mensen in het bijzonder noemen.

Onder andere mijn andere paranimf, Gerben, wil ik graag bedanken. Ik weet dat ik in jouw hoofd nog steeds een student ben die mijn tijd, en jouw belastinggeld, vergooid met een beetje knutselen (aan energiefabriekjes) in een laboratorium, maar ik weet ook dat je altijd voor me klaarstaat. Stefan, ook jij bedankt! Jullie weten beide donders goed wat jullie voor me betekenen. Ik kijk graag uit naar onze gezamenlijke uitjes in de breedste zin van het woord; of het nu de zoektocht is naar net dat unieke biertje wat we nog niet hebben gehad, de beklimming van 
een col zo episch als Pico Del Teide, de moshpit van BZB in Helmond, een ritje met taxi Pol uit Dinant, of de KlapsMühle in Keulen, het kan eigenlijk niet mis. Oh, en tennis is ook leuk natuurlijk. Rens, ik zie je niet zo vaak meer, maar de whisky's smaken er niet minder om en de elpees draaien nog net zo lekker. Ik zal de platenavonden niet vergeten, Space Oddity van David Bowie blijft een van mijn all-time favorieten na het vastloop-incidentje op de Rivierstraat. Natuurlijk ook Albert, Maarten \& Maurice, erg bedankt voor de jaren dat we samen voor de BZB speelde, de vele balletjes die we sloegen en de biertjes die we dronken. Verder wil ik ook alle andere leden van TCE en EST Fellenoord bedanken voor de mooie tijd die ik daar heb mogen spenderen.

Ben, -ik heb het even opgezocht op het moment van schrijven- we verbleven in het laatste jaar 65 uur en 19 min in de gym waarbij we in goede maanden $80.000 \mathrm{~kg} / \mathrm{maand}$ verzette (ik weet het dit getal slaat nergens op). Maar belangrijker nog dan deze reps, vond ik de gesprekken die we konden voeren na goede- en slechte- dagen op het werk. Eigenlijk alles wat me dwarszat op het werk kon ik wel met jou bespreken, en dan was de conclusie vaak dat we ons niet moesten aanstellen en door moesten gaan. Fitness zal het komende jaar wat lastiger worden maar ik hoop dat we toch weer ooit samen in de ijzers zullen hangen. Ook van onze tijd samen in Dubbel Fout heb ik erg genoten, en ik wil daarom ook graag alle huidige en ex-leden van de band bedanken voor onze avonturen samen.

Robin, ik mag je weer landgenoot noemen, \& Frank, we kennen elkaar al heel lang. Elke keer als we weer samen komen dan herinner ik me waarom wij ooit bevriend zijn geraakt al die tijd geleden, het is altijd leuk. Ik hoop dat we weer ooit 'en groupe' beerfest of super troopers zullen bekijken want die films blijven onlosmakelijk met jullie verbonden.

Boys and Girls van het MUMC+ cycling team, ook jullie hebben veel van mij moeten aanhoren tijdens de 270 uur die ik het afgelopen jaar op de fiets besteedde. Jullie hebben moeten luisteren naar alles wat in mijn promotietraject gebeurde tot en met mijn solicitatie-endeavours aan toe, dank daarvoor. Met name de tripjes naar Bad Goisern en Aosta waren een ervaring op zich.

Daarnaast wil ik graag mijn schoonfamilie Anita, Gerard, Esther, Martijn, Floor, Pleun, Frank, Ilse, Fem, Raf \& Mark bedanken voor alle mooie momenten die wij hebben mogen delen. Ik ben erg blij dat ik een plaatsje heb gekregen in jullie familie en hoop dat we nog veel ijsjes zullen eten, spelletjes zullen spelen en dansjes zullen wagen. En eventueel hier en daar een drankje met Carnaval en de kermis in Weert natuurlijk. 
Lilian \& Bram, zonder jullie was ik niet de persoon geweest die ik nu ben. Ik ging in Maastricht wonen en jullie in een 'echte' stad waardoor we elkaar niet zo vaak zien als ik zou willen, en dat zal -door mijn schuld- misschien dit jaar nog wat minder worden. Maar ik ben er trots op dat jullie mijn grote zus en kleine broer zijn en heb nu al zin in de festivalletjes en weekendjes die er allemaal nog aan gaan komen. Ik wil jullie, en ook Leonie -een fantastische aanwinst voor de familie- graag bedanken voor alle tijd die we samen hebben besteed.

Lieve pap \& mam, jullie hebben me alles gegeven wat ik nodig had. Heel veel liefde, kennis, een mooie jeugd, en de voorliefde voor de wetenschap. Zonder jullie was dit boekje er sowieso nooit geweest en ik wil jullie heel erg bedanken voor alles wat jullie ooit voor me hebben gedaan, en nog gaan doen. Deze alinea is te kort om uit te leggen wat jullie voor me betekenen, maar ik ben ongelooflijk blij met alle steun en het vertrouwen wat jullie me geven, zelfs als ik van het verre zuiden naar het nog verdere noorden vertrek.

Judith, ik ben er ongelooflijk trots op dat ik jou mijn vrouw mag noemen en dat jij met mij samen het avontuur in Denemarken aan wilt gaan. Ik heb in jou mijn maatje gevonden wat ik nooit meer kwijt wil. Ook dit heb ik deels te danken aan mijn promotie-onderzoek, want als mijn PhD proposal niet was goedgekeurd waren wij misschien wel nooit getrouwd. Jij bent de drijvende kracht geweest achter het maken van het daadwerkelijke boekje dat je nu in je hand houdt, en ook jij hebt veel te verduren gekregen tijdens de laatste fase van mijn promotie. Zo wilde ik eerst in Toronto werken, toen in Lyon, toen in Schotland en uiteindelijk in Denemarken, en jij vond eigenlijk alles wel prima "als we maar samen zijn!" Ik weet zeker dat wij, samen met Bowie, een mooi gezin worden en het ongelooflijk naar ons zin gaan krijgen en ik kijk erg uit naar alle avonturen die wij samen nog gaan beleven. Wij gaan lekker verder als Dr. \& Dr. Leermakers. 
Publications 
P.A. Leermakers, A.H.V. Remels, M.I. Zonneveld, K.M. Rouschop, A.M.W.J. Schols, H.R. Gosker. Iron deficiency-induced loss of skeletal muscle mitochondria; the role of mitophagy and secretion of mitochondria-containing vesicles. Submitted

P.A. Leermakers, A.E.M. Kneppers, A.M.W.J. Schols, M.C.J.M. Kelders, C.C. de Theije, L.B. Verdijk, L.J.C. van Loon, R.C.J. Langen, H.R. Gosker. Early-onset alterations in mitophagy and mitochondrial biogenesis signalling during skeletal muscle unloading in mice and men. Submitted

P.A. Leermakers\#, A.H.V. Remels\#, R.C.J. Langen, A.M.W.J. Schols, H.R. Gosker. Inflammation-induced alterations in key regulators of mitophagy and mitochondrial biogenesis in murine skeletal muscle. Submitted. "Contributed equally.

Kneppers AEM, Haast RAM, Langen RCJ, Verdijk LB, Leermakers PA, Gosker HR, van Loon LJC, Lainscak M, Schols AMWJ. Distinct skeletal muscle molecular responses to pulmonary rehabilitation in chronic obstructive pulmonary disease: a cluster analysis. J Cachexia Sarcopenia Muscle. 2019 Jan 18.

Kneppers A, Leermakers P, Pansters N, Backx E, Gosker H, van Loon L, Schols A, Langen $\mathrm{R}^{\#}$, Verdijk $\mathrm{L}^{\#}$. Coordinated regulation of skeletal muscle mass and metabolic plasticity during recovery from disuse. FASEB J. 2019 Jan;33(1):1288-1298. "Contributed equally.

Leermakers PA, Schols AMWJ, Kneppers AEM, Kelders MCJM, de Theije CC, Lainscak $\mathrm{M}^{\#}$, Gosker HR\#. Molecular signalling towards mitochondrial breakdown is enhanced in skeletal muscle of patients with chronic obstructive pulmonary disease (COPD). Sci Rep. 2018 Oct 9;8(1):15007. "Contributed equally.

Kneppers AEM, Langen RCJ, Gosker HR, Verdijk LB, Cebron Lipovec N, Leermakers PA, Kelders MCJM, de Theije CC, Omersa D, Lainscak M, Schols AMWJ. Increased Myogenic and Protein Turnover Signaling in Skeletal Muscle of Chronic Obstructive Pulmonary Disease Patients With Sarcopenia. J Am Med Dir Assoc. $2017 \mathrm{Jul}$ 1;18(7):637.e1-637.e11.

Leermakers PA, Gosker HR. Skeletal muscle mitophagy in chronic disease: implications for muscle oxidative capacity? Curr Opin Clin Nutr Metab Care. 2016 Nov;19(6):427-433. Review. 
Curriculum Vitae 
Pieter Arnold Leermakers was born in Eindhoven, the Netherlands, on December 20th of 1988. In 2007, he graduated from secondary school at the Pleincollege vanMearlant in Eindhoven. He first started the Bachelor of Science program of biomedical technology at the Technical University of Eindhoven in 2007, and subsequently switched to the Bachelor of Science program of biomedical sciences at Maastricht University in 2008. After graduating in 2012, he enrolled in the Master of Science program of biomedical sciences of which he graduated in 2014. During his studies, Pieter performed several research projects within the University of Maastricht. The first project was performed at the Department of Surgery titled "The role of MCPIP in a human model of ischemia reperfusion.", a second research project was performed at the Department of Medical Microbiology titled "The role of miR-146a in pulmonary cells infected with human rhinovirus", and the last project of his studies was performed at the Department of Respiratory Medicine titled "The role of mitophagy in the loss of oxidative capacity in COPD".

During this last project, Pieter designed and submitted a grant proposal requesting funding for a PhD project to the NUTRIM graduate programme, which granted him the funds to perform his PhD research at the Department of Respiratory Medicine of Maastricht University. Both the designing of the grant proposal and the execution of the PhD project were performed under the supervision of prof. dr. Annemie Schols and dr. Harry Gosker. The general aim of this project was to investigate if COPD-related manifestations including muscle disuse, systemic inflammation, and more speculatively, iron deficiency, result in the onset of mitochondrial clearance, and consequently in the loss of muscle oxidative capacity. After obtaining his PhD degree, Pieter will continue his research from a more electrophysiological point of view in the Department of Biomedicine of Aarhus University under supervision of dr. Thomas Holm Pedersen. 